(C)[2012]

Eren Erman Ozguven

ALL RIGHTS RESERVED 


\title{
A SECURE AND EFFICIENT INVENTORY MANAGEMENT SYSTEM FOR DISASTERS
}

\author{
by \\ EREN ERMAN OZGUVEN
}

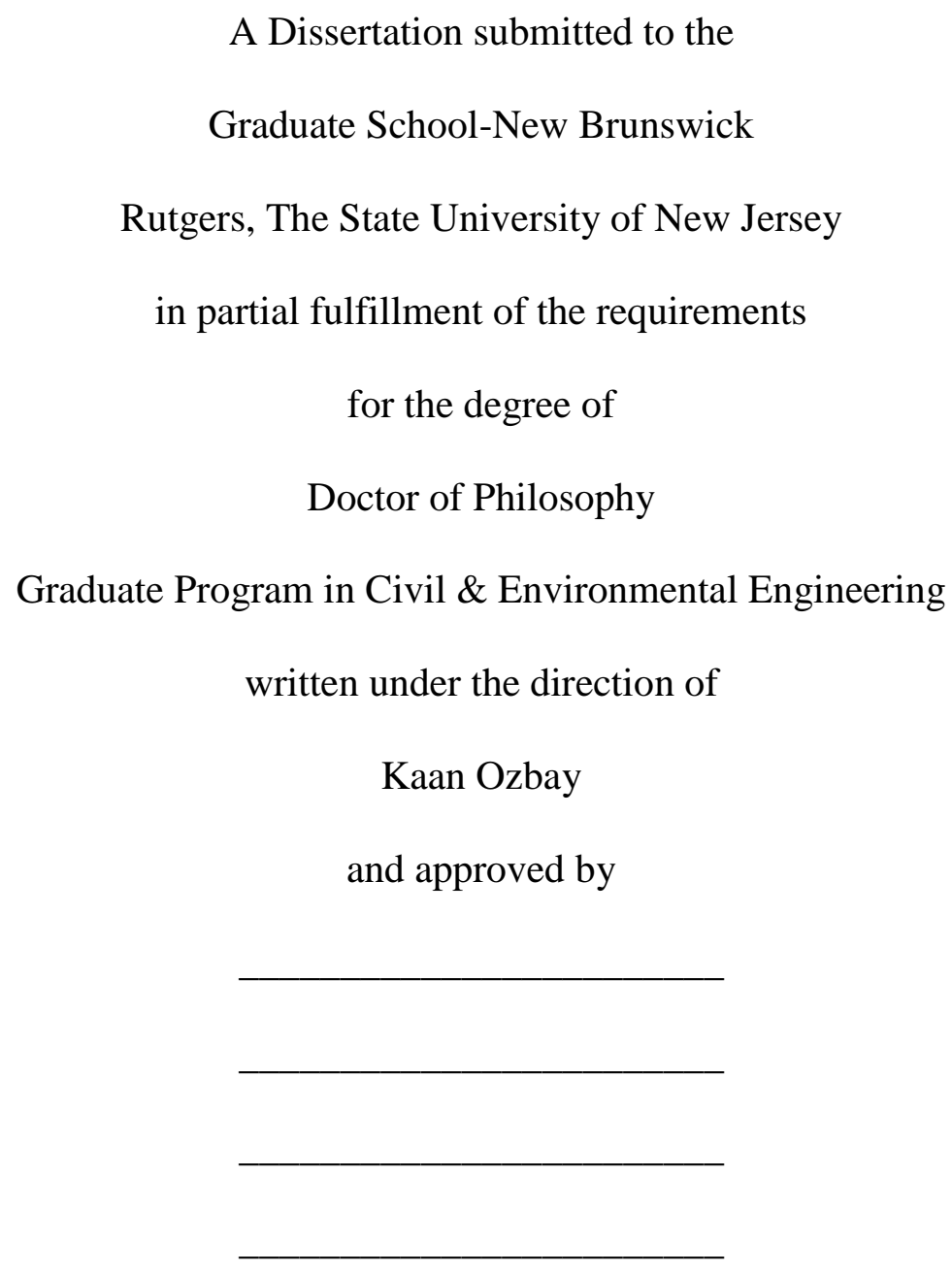

New Brunswick, New Jersey

[May, 2012] 


\section{ABSTRACT OF THE DISSERTATION \\ A Secure and Efficient Inventory Management System for Disasters by EREN ERMAN OZGUVEN}

\section{Dissertation Director: Kaan Ozbay}

Over the last three decades, disasters worldwide claimed more than 3 million lives and adversely affected the lives of at least 1 billion people (Noji, 1997). Regarding the threats posed by these disasters, emergency disaster management has emerged as a vital tool to reduce the harm and alleviate the suffering these disasters can cause to their victims. A significant task of planners involved in emergency disaster management is planning for and satisfying the vital needs of the people located in emergency shelters such as the Superdome in New Orleans.

This thesis proposes a novel and comprehensive framework for the development of a humanitarian emergency inventory management system based on the real-time tracking of emergency supplies and demands through the integration of emerging technologies such as Radio Frequency Identification Devices (RFID) for commodity tracking and logistics. The novelty of this thesis is that, for the first time in the emergency inventory management field, the proposed approach combines an offline planning strategy with online control techniques in a unified framework. Within this framework, the offline planning problem is solved by the stochastic humanitarian inventory 
management approach, whereas the online modeling strategies include the application of neural network-based functional approximation, simultaneous perturbation stochastic approximation (SPSA), and continuous time model predictive control (CMPC) techniques.

Unlike previous studies, the flexibility of the proposed inventory management and control model allows the application of the developed mathematical model to extreme events making online real-time tracking possible. Realistic case studies built using information available from past disasters are used to examine the differences in inventory strategies for different types of disasters based on the impact area and duration of the extreme event. The proposed methodology is also capable of representing and understanding real-life cases where uncertainty and limitations on the inventory levels and flow of supplies can be modeled by introducing different levels of stochasticity and real-life constraints.

The overall findings of this thesis have pointed out that the proposed integrated framework can be efficiently used for emergency inventory planning and inventory control during disaster relief operations without ignoring the real-world uncertainties, fluctuations, and constraints of disaster conditions. 


\section{Acknowledgements}

I would like to express my deepest gratitude to my advisor Dr. Kaan Ozbay for his support and guidance over the course of my Ph.D. study. I would also like to thank my thesis committee members Dr. Maria Boile, Dr. Hani Nassif, Dr. Robert Noland and Dr. Eric Gonzales for their valuable comments that helped me shape this dissertation.

I would also like to thank to my invaluable network of supportive, generous and loving friends without whom I could not have survived the process. I want to specially express my appreciation to Sedam and to my dear friends; my EDKID family and my Rutgers family, especially to my PhD-mates Anil and Ozlem.

Last but not the least, words alone cannot express the gratitude I owe to Cetin Ozguven and Halime Ozguven, my parents, for their love, encouragement and endless support. 


\section{Dedication}

To my parents Cetin Ozguven and Halime Ozguven... 


\section{Preface}

Same as the work conducted in this dissertation has been presented and published in several conferences and journals. Below is the list of publication derived from this dissertation with corresponding chapter numbers.

\section{Chapter-3 \& Chapter-4}

- Ozbay, K., and Ozguven, E. E., (2007) "Stochastic Humanitarian Inventory Control Model for Disaster Planning", Proceedings of the Transportation Research Board's $86^{\text {th }}$ Annual Meeting, Washington, DC, USA.

- Ozbay, K., and Ozguven, E. E., (2007) "Stochastic Humanitarian Inventory Control Model for Disaster Planning", Transportation Research Record, 2022, pp. 63-75.

- Ozguven, E.E, and Ozbay, K., (2012) "Case Study Based Evaluation of a Stochastic Multi-Commodity Emergency Inventory Management Model", Accepted for Publication at Transportation Research Record.

\section{Chapter-3 \& Chapter-4 \& Chapter-5}

- Ozguven, E. E., Ozbay, K., (2009) “A Secure and Efficient Emergency Management System for Disasters", Proceedings of the International Conference on Models and Technologies for Intelligent Transportation Systems, June 22-23, pp. 298-305, Rome, Italy.

- Ozguven, E. E., Ozbay, K., (2011) "A Secure and Efficient Inventory Management System for Disasters", Transportation Research Part C, doi:10.1016/j.trc.2011.08.012. 


\section{Table of Contents}

Abstract of the Dissertation ........................................................................................... ii

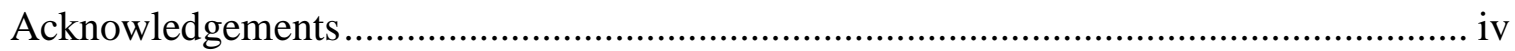

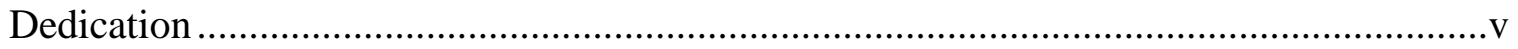

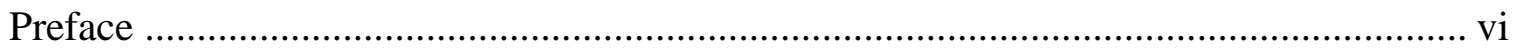

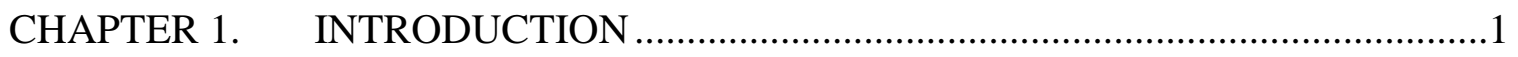

$1.1 \quad$ Problem Definition and Strategy ................................................................

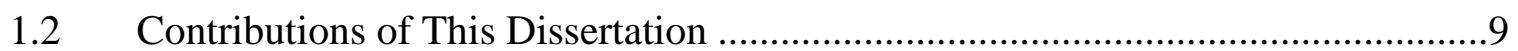

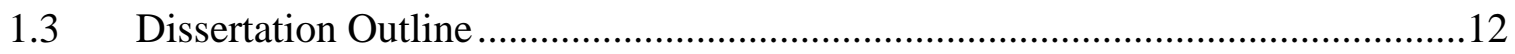

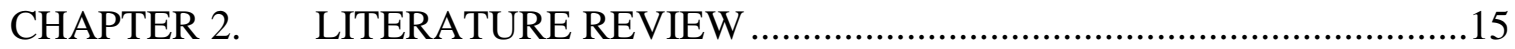

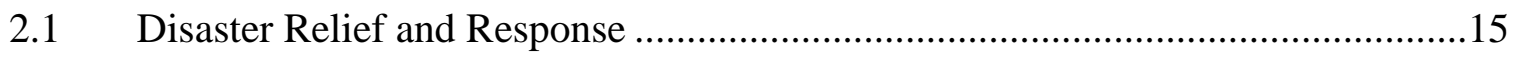

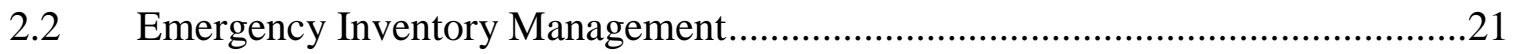

2.2.1 Inventory Control and Location/Allocation ........................................25

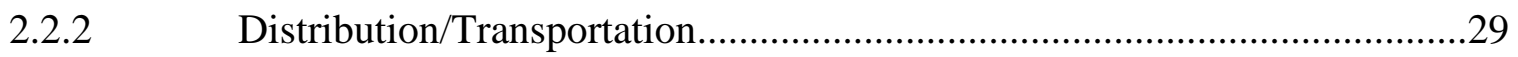

2.2.3 Inventory Control and Location/Allocation Integrated with

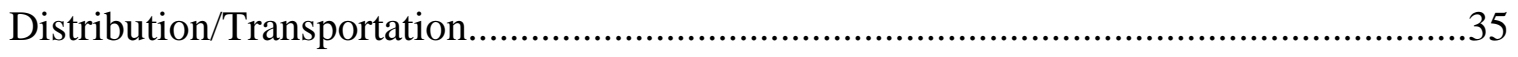

2.2.4 Use of Information Technologies for Emergency Inventory Management.39

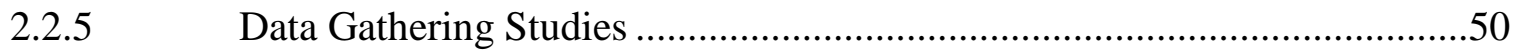

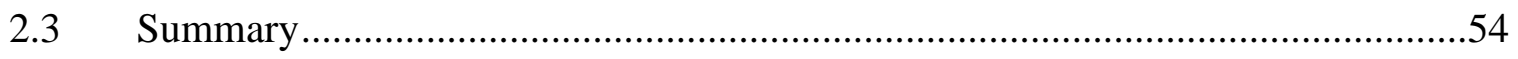

CHAPTER 3. EMERGENCY INVENTORY MANAGEMENT WITHIN THE

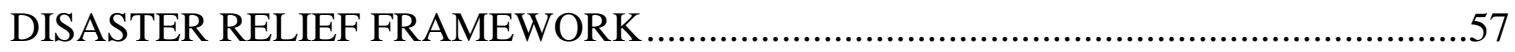

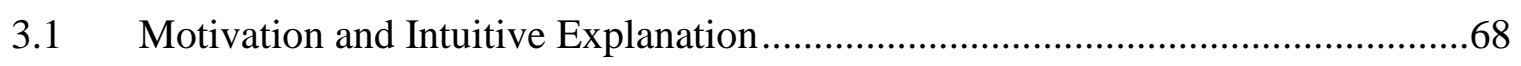

3.2 Possible Application with Radio Frequency Identification Devices (RFID) .......69

3.2.1 Implementation using Our Integrated Emergency Management Model......75

CHAPTER 4. OFFLINE PLANNING: STOCHASTIC HUMANITARIAN

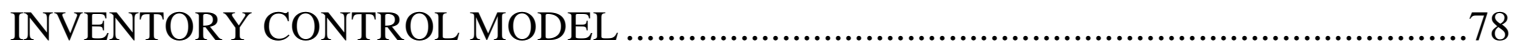

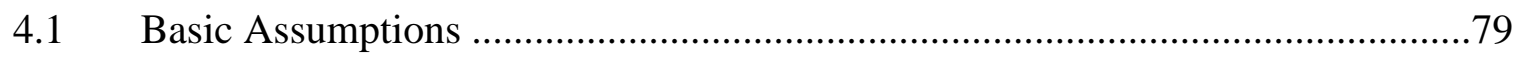

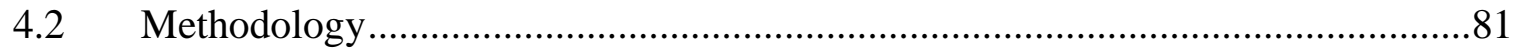

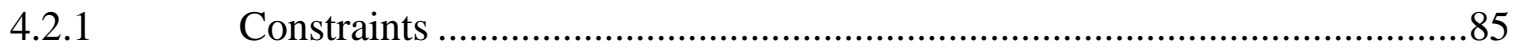




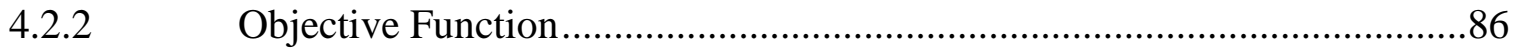

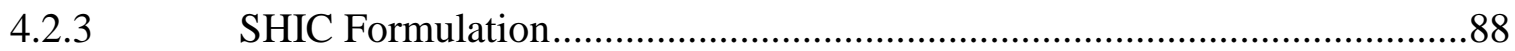

4.2.4 Proposed Solution Approach ................................................................

4.2.5 Prékopa-Vizvari-Badics Algorithm (Prékopa, 2010) ..................................93

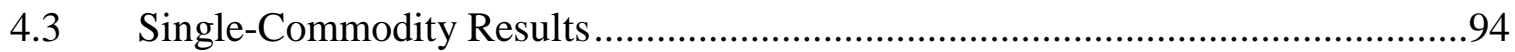

4.3.1 Single-Commodity SHIC Formulation .....................................................94

4.3.2 Single-Commodity SHIC Base Case Scenario ...........................................95

4.3.3 Single-Commodity SHIC Sensitivity Analysis Results ................................97

4.3.3.1 Changes in the Probability of Disruption............................................97

4.3.3.2 Changes in the Initial Safety Stock ..................................................100

4.3.3.3 Changes in the Number of Deliveries ............................................102

4.3.3.4 Changes in the Amount of Consumption.........................................105

4.4 Multi-Commodity Case Studies ....................................................................108

4.4.1 Example Numerical Study for the MC-SHIC Model ................................108

4.4.2 Case Study Information ..................................................................112

4.4.3 Multi-Commodity SHIC Case Studies...................................................114

4.4.3.1 Case Study 1 (Base Case): Change in the Severity of the Disaster .114

4.4.3.2 Case Study 2: Modeling Different Distributions ..............................118

4.4.3.3 Case Study 3: Disruptions to the Transportation Network ...............124

4.4.3.4 Case Study 4: Multi-suppliers or Cross Shipping .............................127

4.4.3.5 Case Study 5: Vital/Perishable Commodities ....................................129

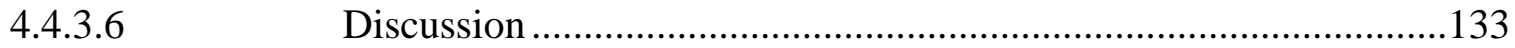

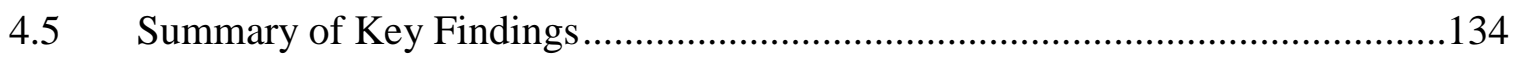

CHAPTER 5. ONLINE CONTROL \& INVENTORY MANAGEMENT ................136

5.1 Model 1: Functional Approximator Approach …………………………….........137

5.1.1 Motivation and Intuitive Explanation ......................................................139

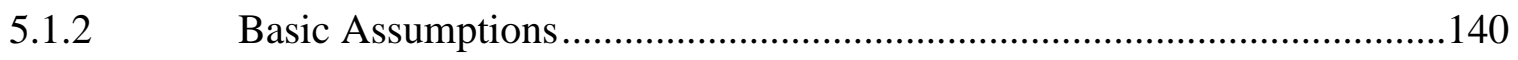

5.1.3 Functional Approximator ..........................................................................141

5.1.3.1 Offline Training Procedure …………..........................................141

viii 
5.1.4 Simultaneous Perturbation Stochastic Approximation (SPSA) Method ...146

5.1.5 Numerical Study: Target Levels Kept Constant .....................................150

5.1.6 Numerical Study: Fluctuating Target Levels Due to Stochasticities .........154

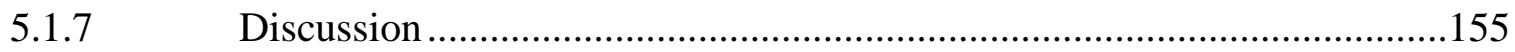

5.2 Model 2: Continuous Time Model Predictive Control Approach ......................159

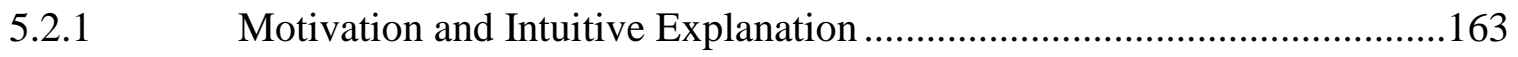

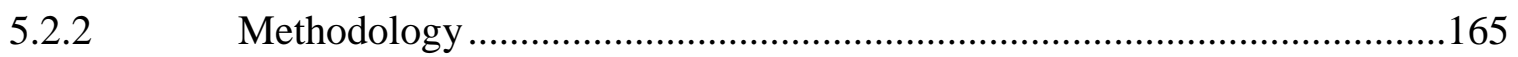

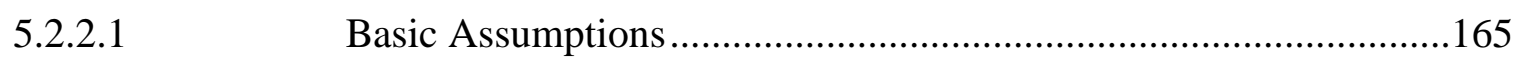

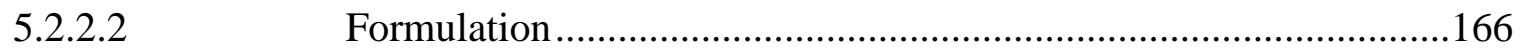

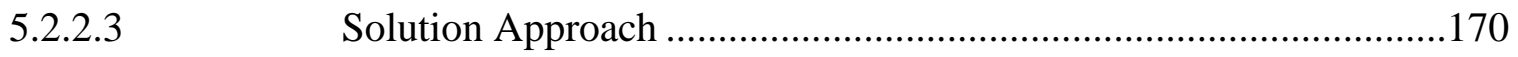

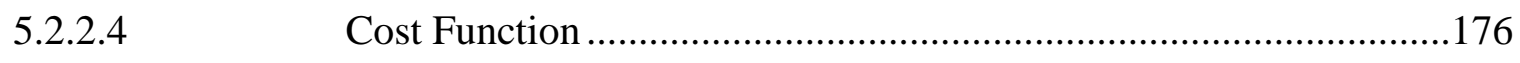

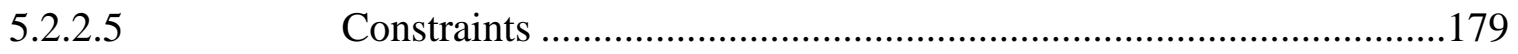

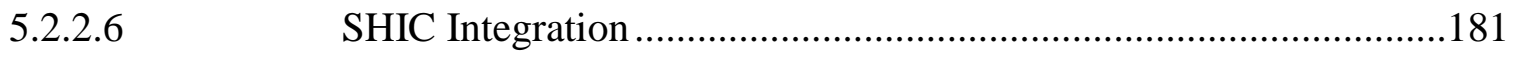

5.2.2.7 Solving the Quadratic Inventory Control Problem (QICP) .............182

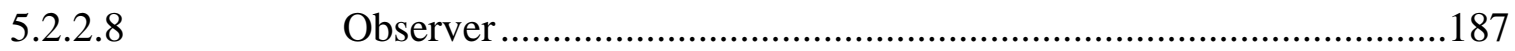

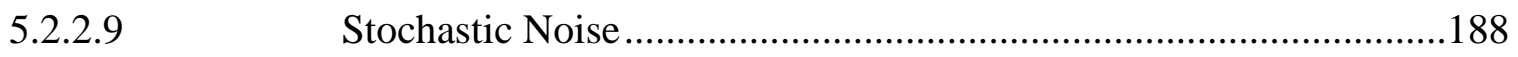

5.2.2.10 Model Tuning Guidelines .........................................................189

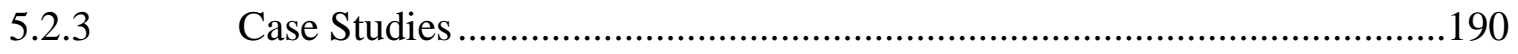

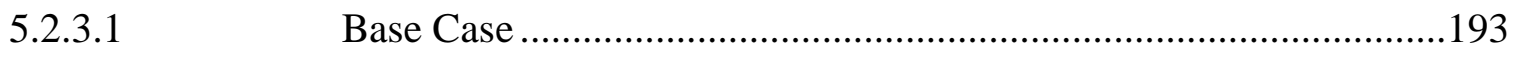

5.2.3.2 Case 2: Increase in the Severity of the Disaster.............................201

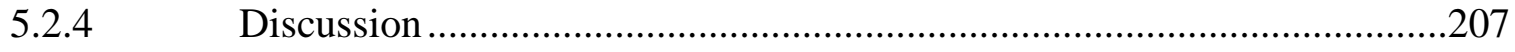

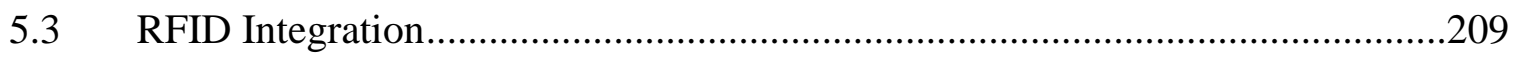

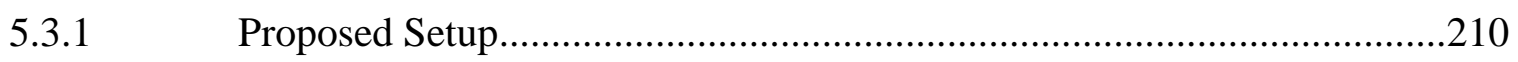

5.3.2 Advantages of the RFID Based Tracking Approach ..............................211

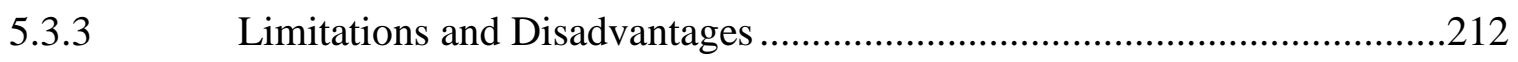

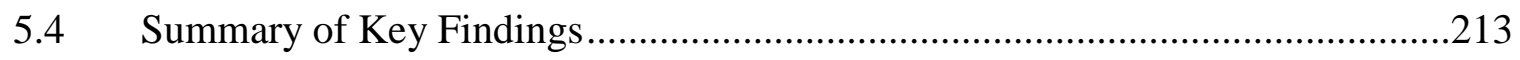

CHAPTER 6. CONCLUSIONS AND FUTURE RESEARCH ............................216

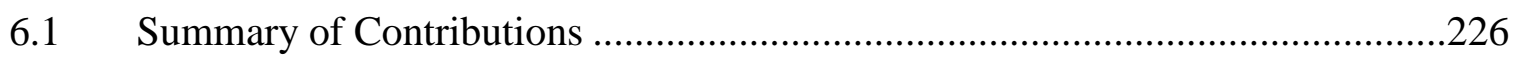




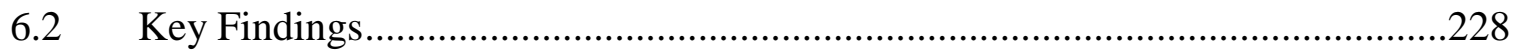

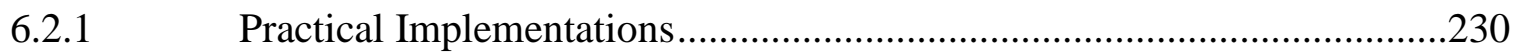

6.2.2 Limitations and Realistic Suggestions to Deal with These Problems........230

6.2.2.1 Discussions of Alternative Approaches .........................................232

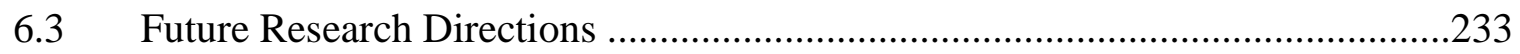

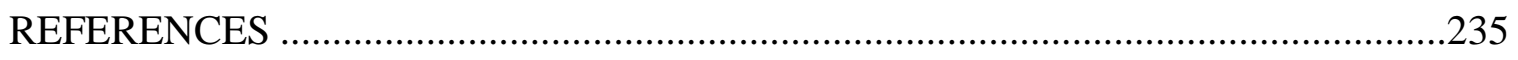

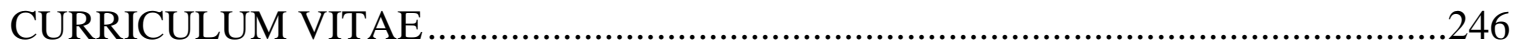




\section{List of Tables}

Table 1.1 Experiences of New Orleans shelter residents (Brodie et al., 2006) ................. 2

Table 2.1 Comparative Literature Review for Emergency Inventory Management ........ 25

Table 3.1 Summary Statistics for Disasters by Type (1900-2011) (http://www.emdat.be)

Table 4.1 Inputs and Outputs for the SHIC Model .................................................... 85

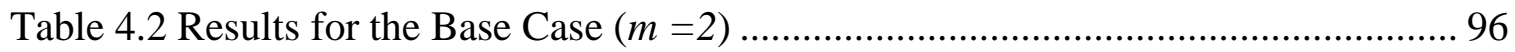

Table 4.3 Results for the Increased Consumption Case ............................................. 106

Table 4.4 Results for the Two-Commodity Numerical Example ................................ 110

Table 4.5 Parameter Information for Case Studies .................................................. 113

Table 4.6 Results for the Two-Commodity Analysis of Category 3 Hurricane ............. 116

Table 4.7 Results for the Two-Commodity Analysis of Category 5 Hurricane ............. 118

Table 4.8 Parameter Selection for Different Distributions of MRE's and Medicine ...... 120

Table 4.9 Comparison for Single and Two-Commodity Analysis for Medicine............ 132

Table 5.1 Inputs and Outputs for the Model-free Feedback Control Methodology ....... 150

Table 5.2 Performance of the Levenberg-Marquardt and SPSA Algorithms for Target

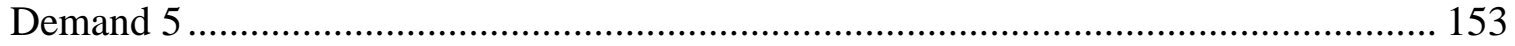

Table 5.3 Performance of the Levenberg-Marquardt and SPSA Algorithms for Target

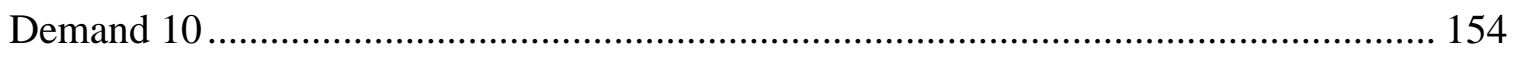

Table 5.4 Performance of the Levenberg-Marquardt and SPSA Algorithms with the

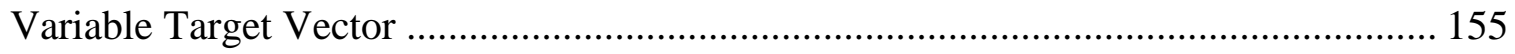

Table 5.5 Inputs and Outputs for the CMPC Model .................................................. 190

Table 6.1 Application of the Proposed Model for Different Types of Disasters ............ 226

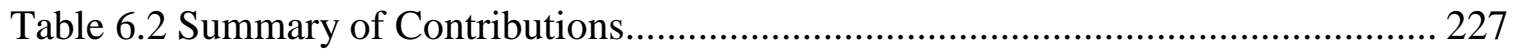

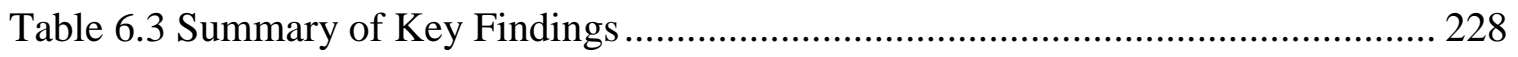




\section{List of Illustrations}

Figure 1.1 Number of Natural Disasters by Country between 1900-2011 (http://www.emdat.be) ......

Figure 1.2 Where Do Humanitarian Inventory Management Problem Stand in the Overall

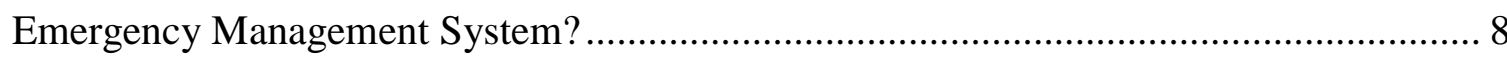

Figure 2.1 Emergency Management Cycle for Disasters (NRC of the National

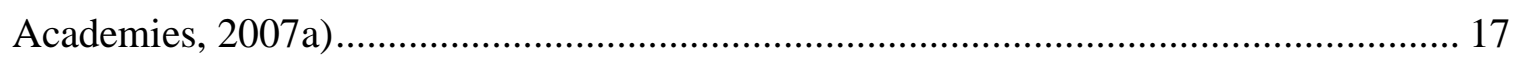

Figure 2.2 Inventory Control and Location/Allocation versus Distribution and

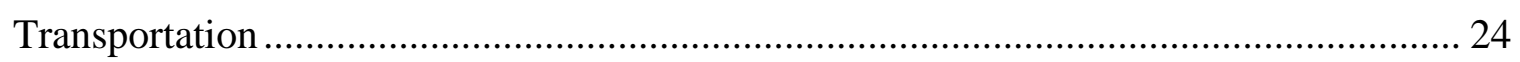

Figure 3.1 Disaster Impact on People: Comparison for 2004 and 2005

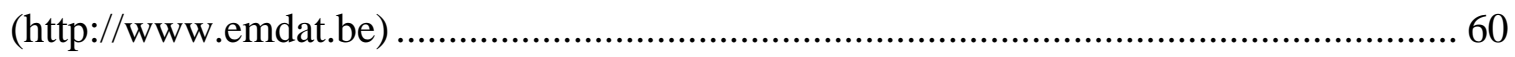

Figure 3.2 A Disaster Shelter (http://www.jhsph.edu/bin/h/e/beds.JPG) ....................... 61 Figure 3.3 Supply of Basic Needs for Disaster Victims

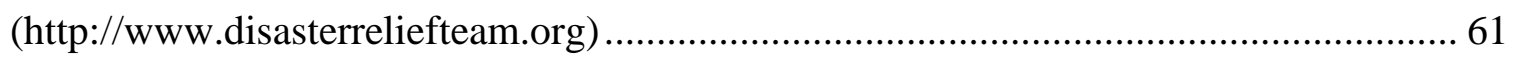

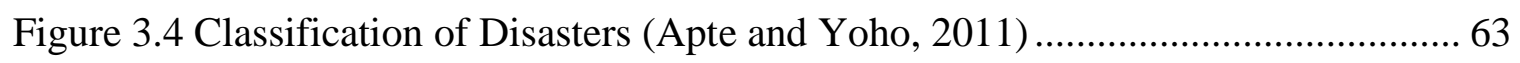

Figure 3.5 Overview of the Emergency Inventory Management Problem ........................ 64

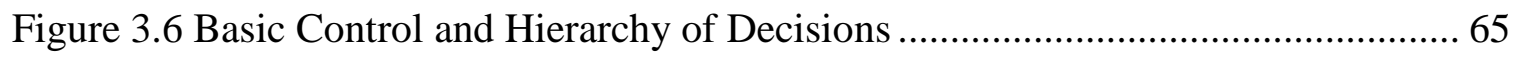

Figure 3.7 Comprehensive Feedback-based Emergency Inventory Control Strategy ...... 67

Figure 3.8 RFID Enabled Procurement of Evacuee Demand in Shelters ........................ 71

Figure 3.9 Four Stages of RFID-based Emergency Management System for Disasters

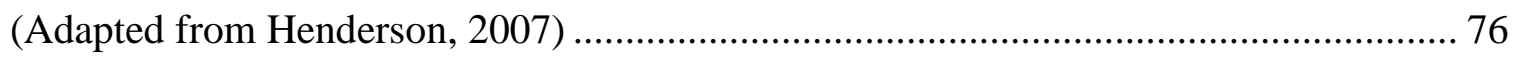

Figure 4.1 pLEPs Method Illustration for Relaxing the Formulation............................. 92

Figure 4.2 Changes in Additional Safety Stock Values (Figure 4.2.a), Occupied Spaces of Safety Stocks (Figure 4.2.b), Total Cost for the Base Case (Figure 4.2.c) and Total Costs for Different Distributions (Figure 4.2.d) for Different Probabilities........................... 100 Figure 4.3 Changes in Additional Safety Stock Values (Figure 4.3.a) and Total Cost

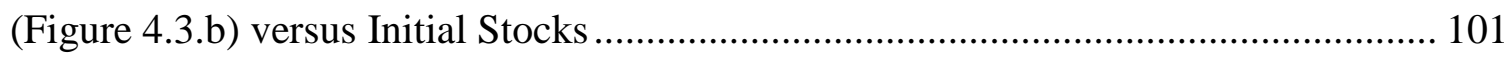


Figure 4.4 Changes in Additional Safety Stock Values (Figure 4.4.a), Total Cost for the Base Case (Figure 4.4.b) and Total Cost for Two Different Distributions (Figure 4.4.c) versus Changes in the Number of Deliveries. 104

Figure 4.5 Changes in Additional Safety Stock Values (Figure 4.5.a) and Total Cost (Figure 4.5.b) versus Changes in Average Consumption Amounts. 107

Figure 4.6 Overall Methodology for the Case Based Inventory Management 111

Figure 4.7 Changes in Additional Safety Stock Values versus Changes in Demand Scenarios for the Hurricane 118

Figure 4.8 Changes in Additional Safety Stock Values for MRE's (Figure 4.8.a) for Medicine (Figure 4.8.b) with respect to Distribution Changes. 122

Figure 4.9 Changes in Additional Safety Stock Values for MRE's (Figure 4.9.a) for Medicine (Figure 4.9.b) with respect to Skewness Changes 124

Figure 4.10 Changes in Additional Safety Stock Values for Medicine (Figure 4.10.a), for MRE's (Figure 4.10.b) and Changes in Total cost (Figure 4.10.c) for Different Number of Deliveries 127

Figure 4.11 Changes in the Additional Safety Stock Values for MRE's via Multi-suppliers 129

Figure 5.1 Model-free Feedback Control Methodology: (5.1.a) Offline Training of the Function Approximator using SHIC Model (5.1.b) Online Control Model based on the Function Approximator and SHIC Model .............................................................. 138

Figure 5.2 Multi-layer Feed Forward Neural Network.................................................. 143

Figure 5.3 RMSE versus Iterations (SPSA Algorithm) ............................................. 158

Figure 5.4 Concept of Receding Horizon (Adapted for the Online Disaster Management Problem from Kwon and Han, 2005) ..................................................................... 162

Figure 5.5 Emergency Inventory Control Framework with the Observer ...................... 188 Figure 5.6 Online Continuous Time Model Predictive Control Results for the Base Case: Results with and without Constraints (Figure 5.6.a) and Comparative Results (Figure 5.6.b) 198 
Figure 5.7 Online Continuous Time Model Predictive Control Results for the Base Case:

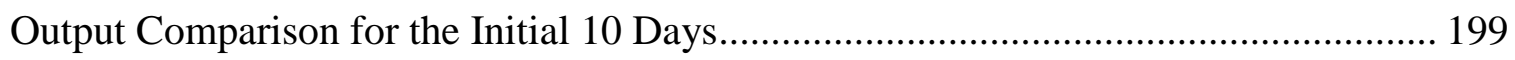

Figure 5.8 Online Continuous Time Model Predictive Control Results for the Base Case:

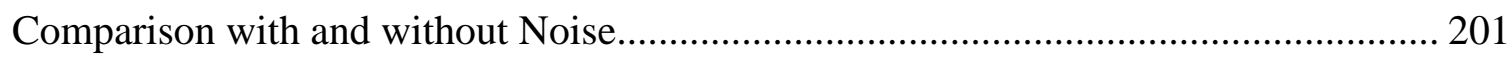

Figure 5.9 Online Continuous Time Model Predictive Control Results for the Case 2: Results with and without Constraints (Figure 5.9.a) and Comparative Results (Figure $5.9 . b)$ 205

Figure 5.10 Online Continuous Time Model Predictive Control Results for the Case 2:

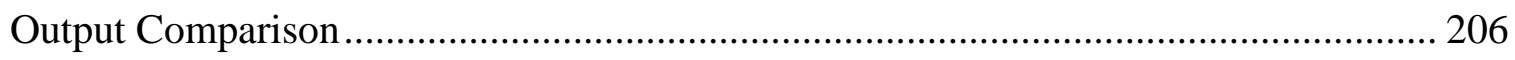

Figure 5.11 Online Continuous Time Model Predictive Control Results for the Case 2:

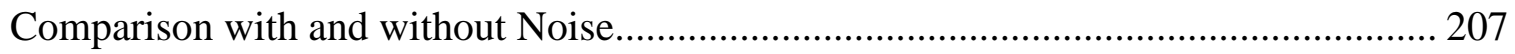




\section{CHAPTER 1. INTRODUCTION}

An elderly man came into our shelter in Waveland. He said that his wife just had surgery yesterday and that they were still staying at their property, even though their home was severely damaged. I asked if there was anything he needed. I still tear up when I remember what he said: "It would be nice if we could have a blanket for my wife."

(Scurfield, 2006)

This real story that happened in the aftermath of Katrina clearly shows the inadequate distribution and availability of vital resources. Moreover, it reveals that the most important issue to consider after a disaster is addressing the basic survival needs of victims. Unfortunately, after Hurricane Katrina, life was not very simple for victims who were trying to meet these survival needs. A study by Holguin-Veras et al. (2007) stated that the lack of an efficient humanitarian inventory control model caused major negative consequences for the disaster victims after Hurricane Katrina. Moreover, Brodie et al. (2006) indicated that survival needs were not effectively satisfied in the shelters after Hurricane Katrina (Table 1.1). According to this study, 56\% of Houston shelter residents were left without adequate food, $54 \%$ were left without adequate water, and $32 \%$ didn't receive adequate medical supplies. In the aftermath of the two devastating earthquakes in Haiti, more than 3 million people were in need of emergency supplies, and in Japan, 350,000 people were reported as becoming homeless and staying in shelters. 
Table 1.1 Experiences of New Orleans shelter residents (Brodie et al., 2006)

Survey Respondents that lived in the New Orleans

Shelters $($ Total $=680)$

\begin{tabular}{lc} 
& $\begin{array}{c}\text { Shelters (Total=680) } \\
(\%)\end{array}$ \\
\hline Spent time inside the New Orleans Superdome & 35 \\
Spent time inside the New Orleans Convention Center & 7 \\
Spent at least 1 day outside & 40 \\
Went without adequate food & 56 \\
Went without adequate water & 54 \\
Went without prescription medicine & 32 \\
Experienced serious health problems & 13 \\
Experienced minor health problems & 19 \\
\hline
\end{tabular}

Clearly, the underutilization and inefficient control of desperately needed resources can put the health and welfare of survivors in great jeopardy. Moreover, the important point is that these catastrophic events make all of us acutely aware of our vulnerabilities to disasters and force us to develop new methodologies for efficient and quick-response inventory management during emergency operations. With this motivation, mathematical modeling of disaster inventory control systems has recently received increasing attention in the literature. These models will help planners and decision makers to understand the nature of the system and how one can plan for and control emergency resources. However, there is still a gap within these studies in terms of including the uncertainties of the disaster in the model and developing an online methodology for real-time control of emergency supplies.

In this dissertation, we focus on two main concepts for disaster inventory management and operations, namely offline (planning) and online management of emergency supplies. First, there is definitely a need for a humanitarian inventory management model developed prior to the occurrence of a catastrophe that can determine 
the safety stock level of emergency inventories that will prevent possible disruptions at a minimal cost. However, just determining these safety stocks beforehand might not be satisfactory for highly stochastic disaster conditions. That is, apart from being prepared for a disaster, online response during the disaster or during the recovery period following the disaster is also crucial. This is due to the fact that the initial planning policy may not adequately capture stochastic real-life conditions for the following reasons:

- needs of survivors can drastically change during disaster relief operations,

- resources available to produce new products may get scarce due to increased consumption or reduced supply levels,

- resource inventories may be damaged, and inventory stocks may be lost due to disaster-related problems such as power outages, and

- a damaged transportation system might not be able to sustain the flow of emergency commodities to shelters.

Therefore, an emergency inventory management system should also include an online operational strategy to minimize the impacts of these unforeseen disruptions, or at least to address the problem at hand as quickly as possible. The real-time management of preparedness activities will affect the actual performance of the response, recovery, and mitigation processes after the disaster.

Based on this information, to have a robust emergency management system, offline planning and production policies should be integrated with an online inventory management strategy to account for the expectations of fluctuating demand for vital supplies (food, water, medical supplies, etc.) and the disruptions in the transportation network during the disaster relief period. This dissertation is motivated by creating such 
an integrated control strategy to ensure the safety and continuity of the flow of the emergency supplies in the aftermath of a disaster. It is important to note that, given this picture of disaster management and emergency response, it is obvious that information technology (IT) in terms of Intelligent Transportation Systems (ITS) provides capabilities that can help planners and engineers grasp the dynamic and stochastic realities in the aftermath of a disaster more clearly, and helps them formulate better decisions more quickly. ITS technologies can address the aforementioned challenges, which require both the careful tracking of vital resources supplied to the shelters and a methodology to incorporate that tracking data into a disaster framework where planning and online management phases are integrated. RFID technology, among other ITS technologies, appears to have critical importance for emergency inventory operations in terms of resource tracking and logistics. A National Research Council of the National Academies report (2007) stated that investments in advanced technologies like RFID tagging used to manage massive supply chains like those of Wal-Mart and FedEx should be pervasively implemented for disaster management operations.

\subsection{Problem Definition and Strategy}

The most important challenge of emergency disaster management and logistics is reducing the harm and alleviating the suffering a disaster causes to its victims. A significant component of this challenge is satisfying the vital needs of the people located in emergency shelters such as the Superdome shelter in New Orleans used after Hurricane Katrina. This requires disaster planners and engineers to find a way to reduce the uncertainties associated with emergency operations, to calculate and compare the 
possible expected costs of the delivery and consumption processes throughout these operations, and therefore to manage the availability and distribution of vital resources.

Before introducing such a methodology to find a solution for these challenges, it is important to focus on the problem at hand. The study titled "Successful Response Starts with a Map: Improving Geospatial Support for Disaster Management" by the National Research Council, published by the National Academies (NRC of the National Academies, 2007a), states that:

1. "Disasters are large, rapid onset incidents relative to the size and resources of an affected jurisdiction.

2. Disasters are uncertain with respect to both their occurrences and their outcomes.

3. Risks and benefits are difficult to assess and compare.

4. Disasters are dynamic events.

5. Disasters are relatively rare."

These statements clearly indicate that disasters are extreme events that can occur with little or no warning. Moreover, Figure 1.1, which depicts the natural disasters that occurred between 1900 and 2011, clearly shows that we are vulnerable to disasters all around the world. Some countries like Turkey, Haiti, Japan, and Indonesia are extremely vulnerable to severe earthquakes that can kill many people and seriously disrupt the daily lives of survivors. Moreover, the Gulf States in the USA frequently experience major hurricanes such as the infamous Hurricane Katrina. In other places, there may be floods, wildfires, drought, famine, epidemics, extreme temperatures, or other natural disasters. 
There is also an increasing need for research on emergency response due to man-made disasters such as nuclear power plant failures, industrial accidents, severe transportation accidents, and terrorist attacks.

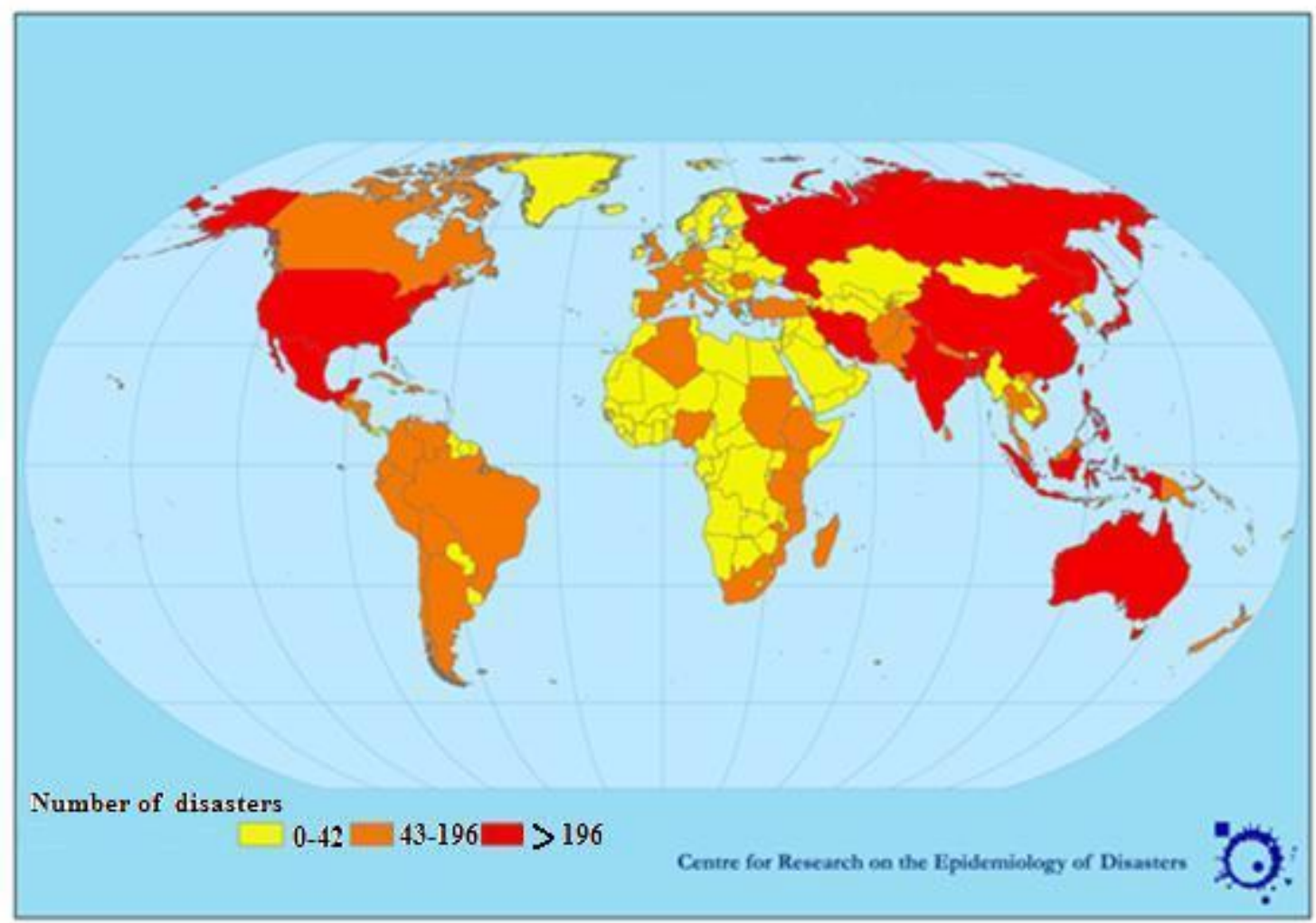

Figure 1.1 Number of Natural Disasters by Country between 1900-2011 (http://www.emdat.be)

Another important issue is the fact that victims may be gathered in shelters during and after a severe disaster. Many of these people, as in the case of Hurricane Katrina, might not have financial resources to leave the disaster area and to find food, medicine, clothes, or other basic needs long after the occurrence of the event. Others might want to stay close to their homes and not want to travel too far, mainly due to a desire to go back to their homes once the threat of the specific disaster is over. Even in the case of Hurricane Irene, which had the strength of Category 1, people were taken to emergency 
shelters following a mandatory evacuation order mainly due to excessive flooding. Therefore, the needs of these evacuees should be satisfied efficiently throughout the entire pre- and post-disaster period. Without an adequate stock of vital commodities, satisfying the daily requirements of these disaster victims without disruptions might be problematic. Due to the severe damage that may be caused by disasters to physical and virtual infrastructure and transportation facilities, even if these needs are available in the stocks, it may not be possible to deliver them to people located in shelters.

One successful example in terms of handling the possible disruptions would be Wal-Mart's performance after Hurricane Katrina. With Katrina approaching, Wal-Mart overstocked its nearby distribution centers with commodities that would be needed the most such as bottled water and generators, and after Katrina struck, Wal-Mart's efficient inventory management and delivery system allowed it to respond quickly to deliver supplies and end the disruption to its supply chain. However, they did not have a mathematical model to support this initial overstocking decision (Leonard, 2005).

To summarize, in this thesis, we are mostly concerned with the effects of disasters on survivors located at shelters. We focus on humanitarian inventory management models in the overall disaster response mechanism to reduce these effects (Figure 1.2). 


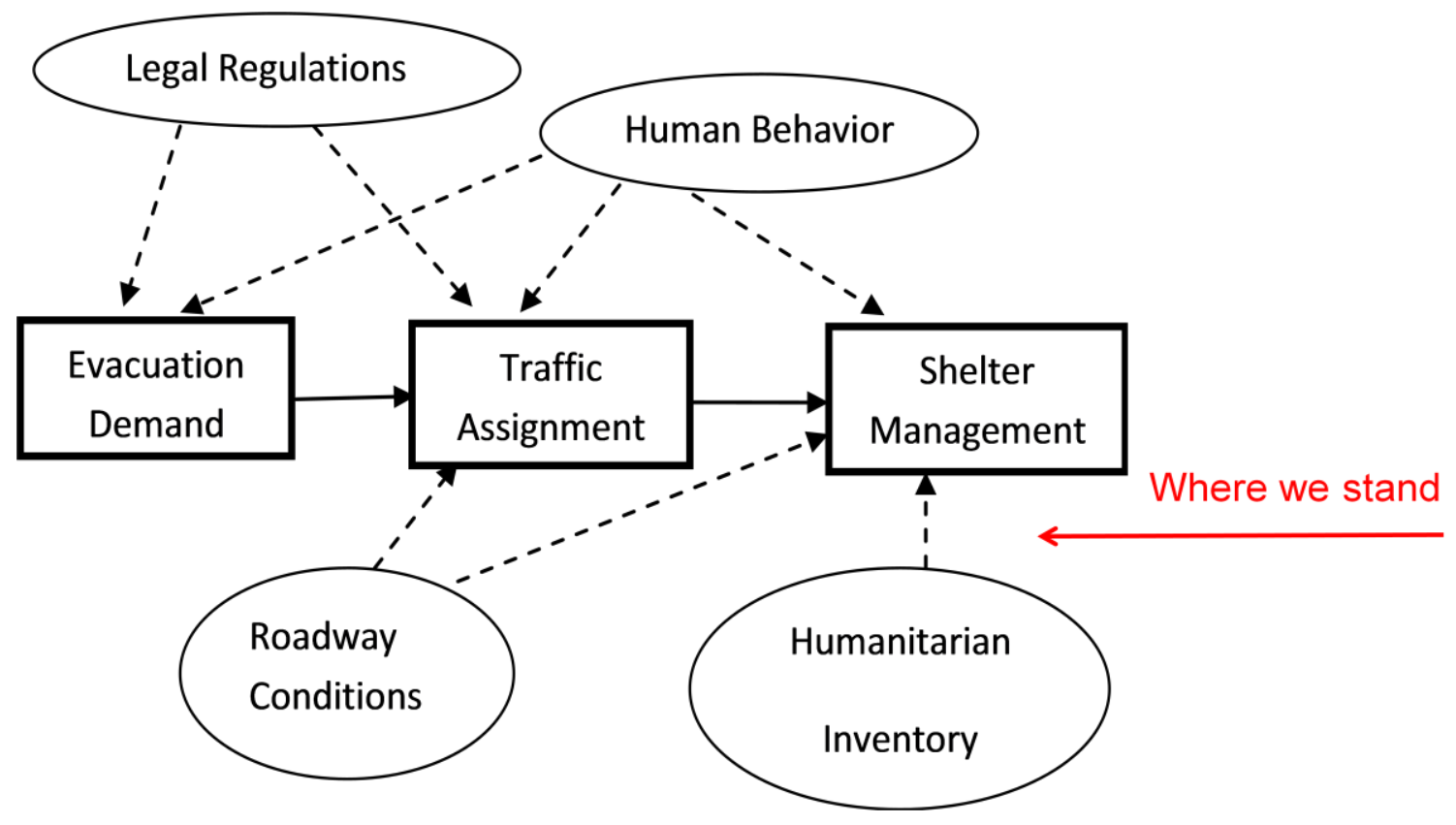

Figure 1.2 Where Do Humanitarian Inventory Management Problem Stand in the Overall Emergency Management System?

Our major questions are:

- How can we most effectively provide assistance to people who have been located in shelters?

- Can sick or hurt people have a chance to receive the medical care they need?

- Can people find enough survival needs (food, water, clothing, etc.) and adequate shelter so that they can survive for a relatively extended period of time after a disaster?

As mentioned in Holguin-Veras et al. (2007), an efficient and reliable flow of critical supplies to the shelters after Hurricane Katrina did not exist, mainly due to the lack of an efficient humanitarian inventory control and a reliable emergency logistics system. This, in turn, created major negative consequences for the survivors of the 
disaster. Holguin-Veras et al. (2007) state that such a system would be difficult due to the following challenges:

- $\quad$ The complex interactions among the dozens of supply chains that arise hamper any formal coordination/optimization process,

- There are no good established procedures to simultaneously handle the material convergence problem and expedite the flow of high priority supplies,

- The lack of empirical studies quantifying the immediate resource requirements hampers the creation of a body of knowledge that could support the estimation of needs after a disaster,

- The transportation system upon which supply chains are supposed to run may be severely damaged."

These statements reveal that an efficient procurement system with an adequate planning strategy and a careful online inventory management policy is a must under highly stochastic disaster conditions. Therefore, the main goal of this dissertation is to obtain an online inventory control framework for vital emergency supplies via integrating an efficient and quick-response inventory management model and feedback control methodologies to adequately respond to a complex emergency situation.

\subsection{Contributions of This Dissertation}

Major contributions of this thesis can be stated as follows: 
1. Unlike previous studies in the literature, the proposed model is designed to successfully address critical and strategic factors associated with humanitarian relief operations to ensure the continuity of unpredictable stochastic delivery and consumption processes. Moreover, the flexibility of the proposed inventory management and control model allows the application of our mathematical model to any extreme event. Our model can account for the flexibility measures of humanitarian relief operations, namely the ability to change the output levels of vital commodities, and the flexibility of their delivery times, defined by Beamon and Balcik (2008).

2. To obtain an efficient and realistic offline disaster inventory plan, the first step is to have a practical yet robust inventory management strategy. This strategy should be closely interconnected with a real-time complex multi-commodity problem that can be implemented and used as a basis for online decision making. Therefore, we model the offline planning model as a multi-commodity and multisupplier stochastic humanitarian inventory control model (SHIC) for which the single-commodity approach was first proposed by Ozbay and Ozguven (2007). We propose a practical and easily applicable solution methodology, namely the pLEPs algorithm (Prékopa, 1990), to solve this multi-commodity SHIC problem so that a decision maker/planner can use the results to decide on the inventory levels for the emergency commodities needed in the shelters. We solve the problem for several realistic case studies built using information available from past disasters to observe changes in the safety stocks, which give the planner a 
self-controlling mechanism to determine these emergency safety stocks more accurately. The proposed multi-commodity SHIC model will aid in adequately responding to a disaster or a humanitarian crisis, focusing on the interdependency among the commodities, transportation of joint resources, limitations on emergency supplies, vital/perishable commodities, multi-suppliers, and crossshipping possibilities.

3. For the online control, we first use a neural network-based function approximator and simultaneous perturbation stochastic approximation (SPSA) algorithm to be able to control highly stochastic and uncertain conditions during extreme events. The target or desired levels of inventory for the functional approximator are calculated using a multi-commodity SHIC model, and the functional approximator is trained to obtain optimal values in the presence of noisy data generated by incorporating the random consumption and delivery distributions of emergency commodities through the multi-commodity SHIC model.

4. We then propose to use a continuous time model predictive control approach (CMPC) that has the ability to compute the trajectory of commodity inventory level changes to optimize the future behavior of disaster inventories based on a receding horizon control approach. Target inventory levels (set point signals) are again calculated using the random consumption and delivery distributions of emergency commodities through the multi-commodity SHIC model. Using these target levels, the CMPC methodology aims to predict the inventory levels for emergency supplies for a predetermined horizon given the information at the 
current time, and given an error-based cost function minimized based on the difference between the desired and the actual inventory levels of the supplies.

5. Our ultimate goal in integrating the offline and online models described above is to develop a model-free closed loop feedback based inventory control strategy that can be effectively applied to different kinds of extreme events, possibly as part of emergency plans and mechanisms, during the disaster relief period. To the authors' knowledge, this has not been done before in the emergency inventory management field. This model-free feedback strategy also requires real-life data about inventory levels, flow of emergency vehicles and supplies as input. RFID technologies, used to keep track of dynamic changes in many commercial inventory systems (such as those of Wal-Mart, FedEx, IKEA, and Procter \& Gamble, which have installed RFID systems in their distribution centers to track commodities coming from suppliers and have reported satisfactory progress with their implementation programs), can be employed to collect this tracking information in real time.

\subsection{Dissertation Outline}

The main consideration in this dissertation is to provide a secure and efficient humanitarian inventory management framework that should be robust with respect to the disruptions that may occur during inventory control and emergency transportation operations in the aftermath of disasters. 
Chapter 2 concentrates on modeling emergency inventory management-related problems by carefully studying offline (planning, pre-positioning) and online (operational) disaster inventory management studies. We focus on the following main categories:

1. Inventory control and location/allocation.

2. Distribution and transportation models.

3. Inventory control and locations integrated with distribution and transportation.

4. Use of information technologies in emergency inventory management operations.

5. Data collection/empirical studies.

Chapter 3 presents the proposed emergency management framework for complex emergency cases such as disasters in a real-time feedback scheme, focusing on the possible use of RFID devices. This RFID-based emergency management and relief system is defined and introduced through a two-step approach with offline planning and online real-time management of emergency management operations.

Chapter 4 focuses on the development and analysis of a novel humanitarian inventory management model developed prior to the occurrence of a natural or man-made catastrophe, which can determine the safety stock level that will prevent possible disruptions at a minimal cost. First, the mathematical formulation and the sensitivity analysis of the humanitarian inventory management problem, first proposed by Ozbay and Ozguven (2007) as the Stochastic Hungarian Inventory Control (SHIC) model, are given. The solutions to single- and multi-commodity cases are presented and discussed. The results for worst-case scenarios are especially emphasized to better understand the robustness of the proposed model under extreme conditions. 
Chapter 5 proposes a robust emergency management framework where offline planning and production policies are integrated with an SHIC inventory management strategy to account for the expectations of fluctuating demand for vital supplies (food, water, medical supplies, etc.), lost inventories, and disruptions in the transportation network during the disaster relief period. Two methodologies are introduced for online control policy within the framework. First, a functional approximator (FA) is proposed to deal with noisy data and to obtain an acceptable control methodology without having knowledge of process dynamics for the nonlinear stochastic emergency relief system. Second, a model predictive control approach (MPC), which has the ability to compute the future trajectory of vital commodity inventories and to optimize the future behavior of the disaster inventory management framework, is proposed.

Chapter 6 presents conclusions, contributions and future tasks. 


\section{CHAPTER 2.LITERATURE REVIEW}

As mentioned in the previous chapter, catastrophic events make us acutely aware of our vulnerabilities to disasters, and force us to develop new methodologies to obtain an efficient and successful emergency inventory management system. In the literature, there are several emergency inventory planning studies with respect to different areas of applications that we will discuss in this chapter. However, it is important to note that there is not much past work on the deployment of online information technologies for disaster inventory management.

First, we will focus on the basic characteristics of disaster relief and response operations using the response and review literature. Next, the methodologies proposed in the literature will be discussed in detail where we focus on the following main categories:

1. Inventory control and location/allocation.

2. Distribution and transportation models.

3. Inventory control, storage and location integrated with distribution and transportation.

4. Use of information technologies in emergency inventory management operations.

5. Data collection/empirical studies.

\subsection{Disaster Relief and Response}

2009 Disaster Reduction Report of UNISDR (United Nations International Strategy for Disaster Reduction) defines the emergency management as: 
"The organization and management of resources and responsibilities for addressing all aspects of emergencies, in particular preparedness, response and initial recovery steps."

Regarding all the disaster types, it is crucial to signify the four phases of an efficient emergency management plan based on the above definition for disasters ((NRC of the National Academies, 2007a)-see Figure 2.1):

- Preparedness: involves activities undertaken in the short term before disaster strikes that enhance the readiness of organizations and communities to respond effectively,

- Response: involves providing emergency assistance to victims,

- Recovery: includes short- and long-term activities undertaken after a disaster for the well-being of people,

- Mitigation: includes those activities, undertaken in the long term after one disaster and before other strikes that are designed to prevent emergencies and to reduce the damage resulting from those that occur, including identifying and modifying hazards, assessing and reducing vulnerability risks, and diffusing potential losses. 


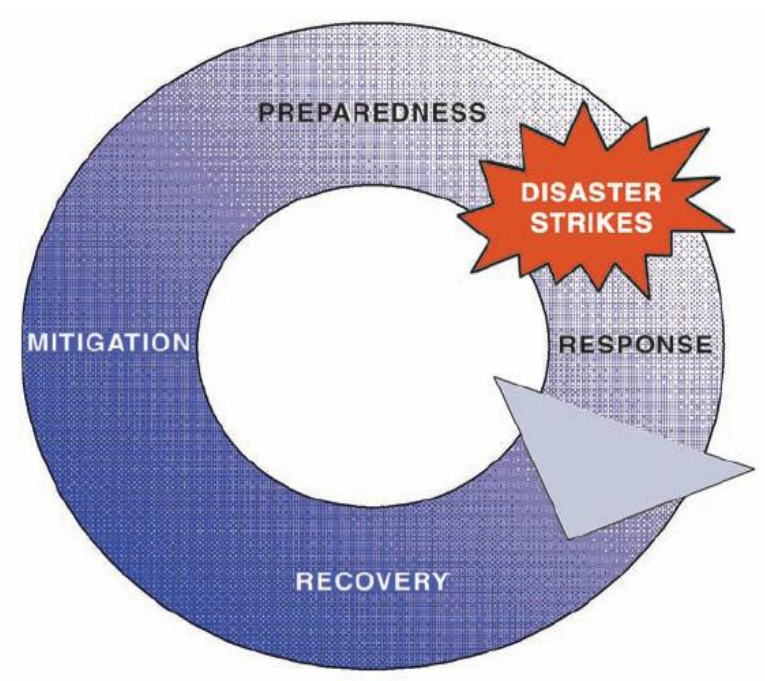

Figure 2.1 Emergency Management Cycle for Disasters (NRC of the National Academies, 2007a)

Humanitarian supply chain models have significant importance while solving the problems related with these emergency management phases. There are several works that study these models in order to increase the efficiency of the emergency response. The first study reviewed in this dissertation includes the work of Long and Wood (1995), who focused on the logistics aspects of famine relief efforts suggesting that having the food available was much more important than holding minimal stock levels. Their most important concern was that the logistical system should support, and not undermine, the social and economic structure of the area. A more wider study has been conducted by Waugh (2000), where the author provided a concise introduction to emergency management and its varied components covering the history and evolution of emergency management and its organization into governmental and regional systems, as well as the management aspects of natural and manmade disasters and possible hazards. By examining the specific cases of both natural disasters and terrorist incidents, this work 
indicated crucial discussion questions that could continue to guide the field and its experts. A similar study was conducted by Rodman (2004). In this work, the author discussed the wide-scale use of supply chain management techniques in the humanitarian sector indicating the importance of supply chain management for humanitarian logistics due to the cost savings and operational efficiencies that could be realized through proper planning, collaboration, and holistic analysis of the relief effort.

A specific review on the humanitarian supply chains for those specifically utilized in the 2004 Southeast Asia Earthquake and Tsunami relief effort was done by Russell (2005). The author created a survey to collect data from organizations involved in Tsunami relief operations instead of relying on anecdotal accounts to identify supply chain issues and opportunities. The data were used to bring to light the logistics problems and inefficiencies, to determine the causes of these issues, and to benchmark current practices. The results of the study indicated that the relief efforts needed more attention on the areas of assessment, collaboration, human resources, and supply chain analysis. Another study included Davidson (2006) who tried to come up with the key performance indicators in humanitarian logistics. The idea was to clearly define the performance indicators and operational targets, and to measure them with the actual performance to these targets. Using this method, the author indicated that organizations would be able to learn from experience and provide a better service to their beneficiaries in the future.

A significant work has been conducted by Blanco and Goetzel (2006), who reviewed the humanitarian supply chain studies defining humanitarian supply chains as the supply chains supporting response to disasters and/or humanitarian crises. According to the authors, response, here, represented preparedness and planning, assessment and 
appeal, mobilization, procurement, transportation, warehousing, and distribution to beneficiaries. Similarly, Ergun et al. (2007) worked on the humanitarian relief logistics including the design of supply chain network, transportation control, and demand management. With this study, the authors started coordination on various research, outreach and educational efforts to improve humanitarian relief planning, capacity building and effective management of response activities. Another study by Sheu (2007) stated the challenges of emergency logistics management in the context of disaster relief and response operations as:

1. Emergency logistics should be redefined as:

"A process of planning, managing and controlling the efficient flows of relief, information, and services from the points of origin to the points of destination to meet the urgent needs of the affected people under emergency conditions."

2. The timeliness of relief supply and distribution is hardly controllable in the emergency context.

3. Resource management for emergency logistics remains challenging.

4. Accurate, real-time relief demand information is required but almost inaccessible. An emergency inventory based review was conducted by Whybark (2007), who stated the important issues considered for the disaster relief, and indicated the need for research in the field of relief inventory management. The study indicated the characteristics of disaster relief inventories, suggesting that what has been learnt from managing enterprise inventories cannot be directly applied to disaster relief inventories, and therefore illustrated the differences between disaster relief inventories and enterprise inventories. 
A key study performed by Beamon and Balcik (2008) defined the flexibility measures of humanitarian relief operations, namely the ability to respond to different type and magnitude of disasters, the ability to change the output levels (to achieve a high level of efficiency), the ability to change the varieties of products (to achieve a high level of effectiveness), and the flexibility of delivery times (to successfully respond to a changing environment). They stated that each relief operation has a different system, so these performance measures should be used as guidelines for varied goals, objectives and operating environments. The emergency management field is also reviewed by Kovacs and Spens $(2007,2011)$ in terms of academic and practitioner contributions for humanitarian logistics, preparation, immediate response and reconstruction related studies, and tried to make a further understanding of logistical operations in disaster relief whereas Altay et al. (2009) examined the stages of disaster management in terms of logistics. They stated that public and private agencies needed to coordinate, integrate and manage the logistics appropriately to meet the performance objectives at the various phases of the disaster relief life cycle (lead-time, quality and cost). In a recent study, Balcik et al. (2010) worked on the coordination in humanitarian relief chains in terms of practices, challenges and opportunities. They stated that the greatest potential among the coordination mechanisms considered were the one that were currently practiced due to their low associated costs, low technological requirements, and being easy to implement and conducive to the relief environment.

After reviewing the response and review literature, we will focus on a core component of the four phases of humanitarian emergency relief for the disasters, namely the inventory control and management. Commodity delivery and consumptions, shelter 
location and allocation, and network logistics for the commodities revolve around it. Therefore, the main emphasis of the next section is to state the characteristics of the emergency inventory management, and clearly indicate the differences between the classical and disaster inventory framework.

\subsection{Emergency Inventory Management}

It is obviously clear that the vital needs of the disaster victims should be supplied efficiently throughout the entire pre and post-disaster period. Adequate stock of vital commodities leads to serious problems in satisfying daily requirements of the disaster victims without disruptions. Moreover, due to the severe damage that may be caused by disasters to the physical and virtual infrastructure and transportation facilities, even if these needs are available in the stocks, it may not be possible to deliver them to victims located in shelters. As mentioned by Holguin Veras et al. (2007), an efficient and reliable flow of critical supplies to shelters after Hurricane Katrina did not exist mainly due to the lack of an efficient humanitarian inventory control model and emergency logistics system. This in turn created major negative consequences for the survivors of the disaster which indicates that efficient methodologies focusing on planning and online inventory management are needed during and/or after disaster conditions.

There are several attempts to obtain such models regarding these issues in the literature, however, compared with the huge enterprise (business) related inventory literature, disaster inventory management literature is not that wide. This is due to the fact that disaster inventory management is unique and significantly different from classical inventory and manufacturing framework due to the following reasons: 
- Disaster inventories cannot be designed like repetitive manufacturing being modeled for daily actions,

- A very common concept, backlogging, cannot be allowed during the emergency operations as delays may even cause deaths in shelters,

- Emergency inventories face a huge amount of uncertain demand, both temporal and spatial in the aftermath of a disaster,

- Online tracking and monitoring the delivery and consumption of the supplies have utmost importance, but too difficult to perform,

- Technological obsolescence for the stocked equipment (i.e., medical apparatus) directly affects the lives of the survivors,

- Perishability and expiration dates of the commodities require extra attention,

- There can be a possible scarcity of resources used to produce new products in the aftermath of a disaster,

- There are monetary, military, local and political constraints in the delivery and storage of the emergency supplies,

- Infrastructure and transportation network can be degraded so badly that efficient delivery of vital supplies cannot be possible.

These crucial points suggest that the special characteristics of the disaster environment clearly distinguish the emergency inventory management from the conventional enterprise inventory management. Therefore, conventional inventory management and control methodologies cannot be directly applied for the disaster operations. This indicates the need for better methodologies, theoretical approaches and management models that should take into account the aforementioned issues. Moreover, 
a major obstacle in all the different areas of emergency inventory management is the presence of uncertainty in the demand and supply operations. Stochasticity involved in the delivery and consumption processes during the disasters should be definitely included in the methodologies. With this idea, uncertainty was introduced into this field in the early 2000's, and they have now become the backbone of the disaster relief and inventory management studies. Recently, several advances in the emergency inventory management have been made that are specifically developed for emergency disaster operations with the help of mathematical programming methodologies in terms of operations research and stochastic optimization.

This section reviews the emergency inventory management studies while focusing on two main components that should be considered for an effective emergency inventory management: inventory control (storage, location, planning, and pre-positioning) and distribution (transportation, multi-suppliers). Figure 2.2 shows these studies in a chronological order with respect to their overlapping application areas whereas Table 2.1 gives the comparative review of these studies with brief description of the work. 


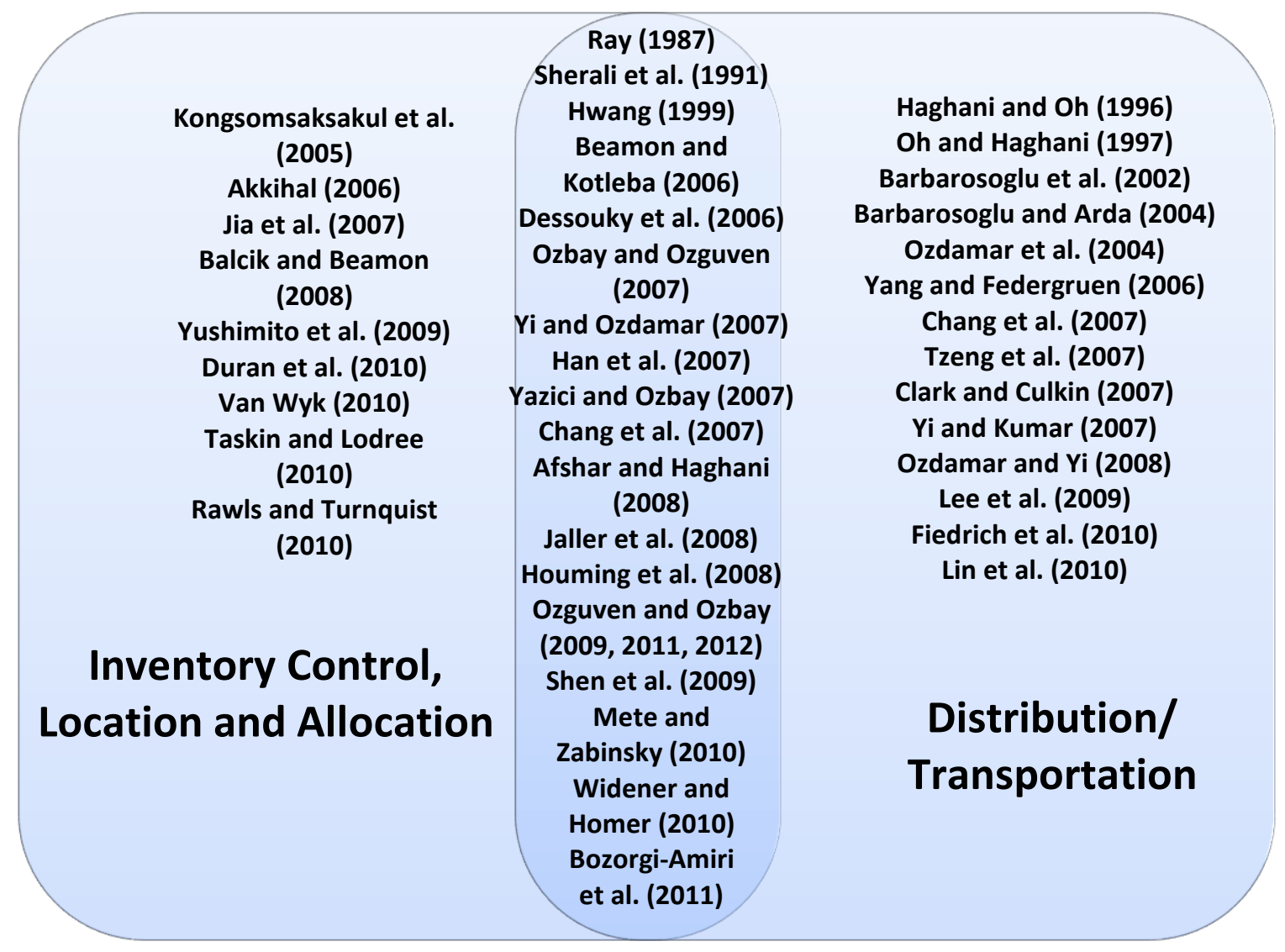

Figure 2.2 Inventory Control and Location/Allocation versus Distribution and Transportation 
Table 2.1 Comparative Literature Review for Emergency Inventory Management

\begin{tabular}{|c|c|c|}
\hline Field & Studies & Description of Work \\
\hline \multirow{2}{*}{$\begin{array}{l}\text { Inventory } \\
\text { Control }\end{array}$} & $\begin{array}{l}\text { Van Wyk (2010), Taskin and Lodree (2010), } \\
\text { Rawl and Turnquist (2010), Lodree (2011) }\end{array}$ & $\begin{array}{l}\text { Prepositioning of Vital } \\
\text { Supplies }\end{array}$ \\
\hline & $\begin{array}{c}\text { Akkihal (2006), Jia et al. (2007), Balcik and } \\
\text { Beamon (2008), Yushimito et al. (2009), } \\
\text { Duran et al. (2010) }\end{array}$ & $\begin{array}{l}\text { Allocation of Shelters } \\
\text { and Inventories }\end{array}$ \\
\hline \multirow[t]{2}{*}{$\begin{array}{l}\text { Distribution and } \\
\text { Transportation }\end{array}$} & \begin{tabular}{|c|} 
Haghani and Oh (1996), Oh and Haghani \\
(1997), Barbarosoglu and Arda (2004), Yang \\
and Federgruen (2006), Chang et al. (2007), \\
Tzeng et al. (2007), Clark and Culkin (2007), \\
Lee et al. (2009), Lin et al. (2010), Fiedrich et \\
al. (2010)
\end{tabular} & $\begin{array}{l}\text { Transportation of Vital } \\
\text { Supplies }\end{array}$ \\
\hline & $\begin{array}{l}\text { Barbarosoglu et al. (2002), Ozdamar et al. } \\
\text { (2004), Yi and Kumar (2007), Ozdamar and Yi } \\
\text { (2008) }\end{array}$ & $\begin{array}{c}\text { Emergency/Rescue Vehicle } \\
\text { Operations }\end{array}$ \\
\hline \multirow{2}{*}{$\begin{array}{c}\text { Inventory } \\
\text { Control with } \\
\text { Distribution and } \\
\text { Transportation }\end{array}$} & $\begin{array}{c}\text { Ray (1987), Beamon and Kotleba (2006), } \\
\text { Ozbay and Ozguven (2007), Jaller et al. } \\
\text { (2008), Ozguven and Ozbay (2009, 2011, } \\
\text { 2012), Mete and Zabinsky (2010), Bozorgi- } \\
\text { Amiri et al. (2011) }\end{array}$ & $\begin{array}{l}\text { Prepositioning and } \\
\text { Transportation of Vital } \\
\text { Supplies integrated }\end{array}$ \\
\hline & $\begin{array}{l}\text { Sherali et al. (1991), Hwang (1999), } \\
\text { Kongsomsaksakul et al. (2005), Dessouky et } \\
\text { al. (2006), Han et al. (2007), Yazici and Ozbay } \\
\text { (2007), Yi and Ozdamar (2007), Afshar and } \\
\text { Haghani (2008), Houming et al. (2008), Shen } \\
\text { et al. (2009), Widener and Homer (2010) }\end{array}$ & $\begin{array}{l}\text { Allocation of Shelters } \\
\text { and Inventories and } \\
\text { Transportation of Vital } \\
\text { Supplies integrated }\end{array}$ \\
\hline
\end{tabular}

In the following subsections, the related literature will be studied extensively. Since some of the studies focused on more than one component, they will be reviewed in a different subsection stating the overlapping parts.

\subsubsection{Inventory Control and Location/Allocation}

This is the most critical component of the overall emergency inventory management process. Unavailability of sufficient amount of emergency supplies for the 
disaster victims will result in serious problems, and can even lead to death. Basically, uncertainty involved in demand and supply processes makes it difficult to be able to obtain an efficient methodology. Short lead or reaction times to satisfy the needs of victims can also dramatically affect the inventory storage and distribution processes.

There are several studies that try to find optimal solutions to these problems in the literature. Among these studies, Van Wyk (2010) proposed two generic mathematical models that could be used to effectively determine the types and quantities of aid supplies required in pre-positioning facilities specifically for the South African Development Community (SADC). A Disaster Recovery Plan (DRP) model was formulated to enhance the survival capability of people in the region directly after the occurrence of any of the disasters identified, and a stochastic inventory model was presented to minimize the total cost of the aid supplies kept in inventory. Similarly, Taskin and Lodree (2010) explored a stochastic inventory problem for manufacturing and retail firms who faced challenging procurement and production decisions in which predictions associated with the ensuing hurricane season demand distribution evolved according to a Markov chain. Basically, they presented a control policy in which stocking decisions were based on a multi-period stochastic programming inventory control problem and a hurricane forecast model that predicted the number of landfall hurricanes for an ensuing hurricane season. Another study on the emergency inventory management was performed by Rawls and Turnquist (2010), who proposed a two stage stochastic integer programming approach to provide a means for a pre-positioning strategy for disasters. They used a heuristic algorithm to solve their problem where disaster-related stochasticities in the demand and availability of the transportation network were included in the model. Recently, Lodree (2011) 
developed a pre-storm planning model for emergency supplies focusing on the volatility of storm forecasts and corresponding demand predictions, and incorporated a minimax decision approach to solve the problem. The weakness of the model was that the model should be extended according to the relevance of each assumption on specific real-world inventory systems.

Determining the optimal locations of shelter and inventory (warehouse) locations, and their proximities to each other also requires a significant effort during the application of mathematical programming techniques for the disaster operations. For this purpose, Yushimito et al. (2009) developed a Voronoi diagram-based algorithm to locate a finite number of facilities to provide a quick response time for disaster relief. Their objective was to locate facilities which will minimize the response time where all demand points in the affected area could be reached from a given facility in the minimum time possible. Jia et al. (2007) also worked on the facility location problem, but they only considered the medical facilities. They used a covering model, namely a P-median model or a P-center model, each applying for distinct characteristics and needs of a large-scale emergency. They illustrated several examples on how to apply their methodology for optimizing the locations of medical facilities to address large-scale emergencies in the Los Angeles area. Similarly, Balcik and Beamon (2008) worked on the facility location problem for humanitarian relief chains and developed an analytical approach that will enable relief practitioners to make efficient and effective facility location and to stock pre-positioning decisions. Their model enabled determining the number and locations of the distribution centers in the relief network, and the amount of relief supplies to be stocked at each center. Akkihal (2006) also examined the impact of inventory pre-positioning on 
humanitarian operations via identifying the optimal locations of inventories for the initial disaster relief and working on a model where the aim was to minimize the average distance of the homeless to the nearest inventory. Historical data for the homeless people obtained during the disasters were used as the weights for distinct demand locations. However, the model assumed that inventories always had enough vital commodities to satisfy the demand of victims, which did not reflect the real life situations. Recently, Duran et al. (2010) worked on a model for pre-positioning the emergency commodities for one of the largest humanitarian organizations in the world, namely CARE International. They evaluated the effect of pre-positioning these items on CARE's average emergency response time to provide relief aid to people affected by natural disasters which could help CARE's managers to determine a desired configuration for the pre-positioning network.

There are, to the authors' knowledge, no application of MPC techniques and neural networks specifically for emergency inventory management. Therefore, we will focus on some example applications of MPC and neural networks for classical industrial inventory management. Theoretically, model predictive control (MPC) strategies serve a wide range of control methods that can be used in supply chain management of emergency evacuation. Braun et al. (2003a, 2003b) showed that an MPC framework could handle demand networks efficiently with robust management. They used a discrete control scheme and applied the method on a case study from semiconductor manufacturing. Other significant predictive control studies include Rasku et al. (2004) and Dunbar and Desa (2005). Rasku et al. (2004) worked on an implementation to obtain a better cost function in inventory control and supply chain management problems, 
whereas Dunbar and Desa (2005) demonstrated the application of a recently developed distributed implementation of nonlinear MPC (NMPC) to the problem of dynamic management of supply chain networks. Other examples of MPC applications include Schwartz et al. (2006), Wang et al. (2007), and Schwartz and Rivera (2010), who worked on model predictive control (MPC)-based decision policies for inventory management in supply chains under conditions involving supply and demand uncertainty. With the help of case studies on semiconductor manufacturing, these studies have shown the efficiency of MCP methodologies for inventory management.

\subsubsection{Distribution/Transportation}

Another important function of the emergency inventory system should be not only to stock inventories of supplies needed, but also to receive and distribute them. For example, After the 2005 Indian Tsunami disaster, Athukorala (2005) stated that the transportation network in the region of Indonesia affected by this disaster was disrupted by around $31 \%$, indicating the importance of the feasibility of the transportation operations. Therefore, due to the severe damage that may be caused by disasters to the physical and virtual infrastructure, and transportation facilities, even if these needs in terms of vital supplies are available in the stocks, it may not be possible to deliver them to victims located in shelters.

On August 28, 2005, the Louisiana National Guard delivered seven truckloads of MRE's to the largest shelter in New Orleans, the superdome, enough to supply 15000 people for three days. This was not enough for the 20000 victims located in the superdome. This indicates that, during the recovery period after the disaster, the 
emergency, rescue and commodity supply activities should be able to take place to satisfy the basic needs of people even if the transportation network is heavily damaged or degraded. Transportation of joint resources, i.e., food and water, is also a major concern as the emergency vehicles supplying shelters may be limited due to these disruptions in the transportation network and unavailability of the personnel and vehicles. Moreover, multi-suppliers may be needed for the same commodity as there may not be enough commodities that can be distributed to the shelter from the usual supplier, or deliveries may not be possible from one supplier due to the damaged traffic network. The communications and infrastructure network may also be down, therefore transportation and supply information can be unreliable, incomplete, or non-existent.

To overcome these problems, several emergency planning models have been developed to focus on the delivery of the vital products during the disasters. First of all, Haghani and Oh (1996) studied the transportation of many different goods, such as food, clothing, and medical supplies to minimize the number of casualties and to maximize the efficiency of the rescue operations. They formulated and solved a multi-commodity, multi-modal network flow model. This model was constructed to have four nodes, five arcs and three transportation modes. Two heuristics were developed, with the first utilizing a Lagrangian relaxation approach and the second employing an iterative fix-andrun process. Oh and Haghani (1997) tested this optimization model extensively with the aforementioned two heuristics. They stated that both solution procedures proved to be efficient in providing close to optimal solutions. Barbarosoglu and Arda (2004) extended the model of Haghani and Oh (1996) into a two-stage stochastic programming model where they included uncertainties due to the estimation of resource needs of first-aid 
goods, vulnerability of resource suppliers and survivability of the routes in the disaster region. They defined the two stages with respect to information asymmetry, which disclosed uncertainty during the progress of the response. The approach was validated by quantifying the expected value of perfect and stochastic information in problem instances generated out of actual data. Another approach was based on a simulation analysis conducted by Lee et al. (2009) to obtain the distribution of emergency relief supplies, where they simulated the supply chain of relief supplies, the distribution operations, and the analytics for the optimal transportation of these supplies to various points of distribution. Their model was developed to analyze a wide range of disaster scenarios, assess the existing disaster response policies, and identify the best approaches to respond for the future disasters.

Mathematical and operations research techniques used to solve the supply distribution problems include integer programming, stochastic programming and network flow models. Among these models, Lin et al. (2010) proposed an integer programming based logistics model for the delivery processes during the disaster relief operations, and applied the model on a simulated earthquake scenario whereas Fiedrich et al. (2000) suggested a mathematical model allowing for calculating an optimized resource schedule for assigning resources in space and time to the impact areas of the disaster. Clark and Culkin (2007) developed a transshipment network flow model to assist policy makers and planners to design an effective supply chain so that goods and service could reach those in need as quickly as possible. They validated their model with a small data set, based on real life data from the South Asian Earthquake of October 2005. Similarly, Tzeng et al. (2007) created a multi-objective mathematical model for designing relief delivery 
systems. They derived a relief commodity distribution model focusing on three aspects: economical (minimal total cost), effective (shortest total travel time), and fair (maximal satisfaction of fairness). Then, they presented an empirical case to demonstrate the feasibility and effectiveness of this model on Taichung, Nantou City, which experienced a major earthquake on September 21, 1999. In a different approach, Yang and Federgruen (2006) developed disaster planning models focusing on selecting suppliers by analyzing a planning model covering uncertain demand from multiple suppliers. They selected the planning problem as which of the given set of suppliers to retain, and how much to order from each, so as to minimize total procurement costs, while ensuring that uncertain demand was met with a given probability. They developed two approximate methods to solve the problem where Central Limit Theorem (CLT) based application gave important insights. Flood emergency logistics was studied by Chang et al. (2007) who used as a GIS-based stochastic programming model including uncertainty to formulate the problem. They aimed at developing a decision-making tool which could potentially be used by disaster prevention and rescue agencies for planning flood emergency logistics preparation. The decision variables in their model included the structure of rescue organizations, locations of rescue resource storehouses, and allocations of rescue resources under capacity restrictions. They explored how to optimize the expected performance over all scenarios and how to incorporate scenario planning into flood emergency logistics preparation modeling and they applied the model to Taipei City, located in Northern Taiwan.

There are also several studies that specifically focused on the emergency/rescue vehicle operations. Ozdamar et al. (2004) presented a mathematical model for emergency 
logistics planning during disasters. They stated that logistics planning in emergency situations involved dispatching commodities (e.g., medical materials and personnel, specialized rescue equipment and rescue teams, food, etc.) to distribution centers in affected areas as soon as possible so that relief operations could be accelerated. They developed a planning model that could be integrated into a natural disaster logistics decision support system. In a similar study, Ozdamar and Yi (2008) used greedy neighborhood research for maximizing service for available emergency vehicles to disaster-areas survivors. They developed Path-Builder, a simple, fast constructive heuristic for identifying a feasible, acceptable solution, and tested its performance on 28 randomly generated test problems constructed on grid networks with integer arc travel times. Another interesting application was proposed by Yi and Kumar (2007), who presented an ant colony optimization (ACO) method to solve the logistics problem arising in disaster relief activities. Their aim was to minimize the delay during the procurement of basic survival needs to survivors and emergency care to injured victims. The only work that focused on the air transportation during the emergency relief operations was conducted by Barbarosoglu et al. (2002), who studied using helicopters for delivering supplies and saving victims during the disasters. They presented a hierarchical multi-criteria modeling perspective that will form the theoretical basis for developing fast planning procedures to be used during the initial response phase.

Destro and Holguin-Veras (2011) worked on a different aspect of the material convergence problem for the disasters (movement of goods to the disaster site) and performed an econometric modeling of the donations made in the case of Hurricane Katrina. The econometric model revealed that, for the disaggregate case, the important 
socio-economic variables that played a role in explaining the amount of donations, were: geographical proximity to the disaster (distance), income, education, unemployment, and type of donor (e.g., organization headquarters, company and individual). Another interesting result of their analysis was that the results indicated the existence of a shift towards monetary donations as distance increased.

The usage of neural networks for transportation during the emergency evacuation is also very limited. One comprehensive study has been done by Mei (2002) where a detailed analysis of artificial neural network models was performed and alternative trip generation models for hurricane evacuation movement using logistic regression and neural networks were developed. With this idea, Wilmot and Mei (2004) estimated models with various forms of neural networks and tested the models with a data set of evacuation behavior collected in southwest Louisiana following Hurricane Andrew. Recently, Danistan and Vipulanandan (2010) worked on an artificial neural network approach to predict the traffic flow for hurricane evacuation focusing on local roads with speed limits, and applied their model on a hypothetical roadway network. As for MPC technologies, on the other hand, one application has been shown by Liu et al. (2007) where they proposed a model reference adaptive control based real-time traffic management framework for the emergency evacuation including both dynamic network modeling and adaptive control theory techniques. 


\subsubsection{Inventory Control and Location/Allocation Integrated with Distribution/Transportation}

Humanitarian inventory control models integrated with an effective distribution methodology are needed to aid in adequately responding to a disaster or a humanitarian crisis. Several researchers recognized this issue, and worked on an integrated inventory control and distribution strategy. The first work recognizing this need was proposed by Ray (1987), who developed a single-commodity, multi-modal network flow model on a capacitated network over a multi-period planning horizon to minimize the sum of all costs incurred during the transport and storage of food aid in West Africa. Another important study was conducted by Beamon and Kotleba (2006), who developed an inventory control model to determine the optimal order quantities and reorder points for a long-term emergency relief response. The proposed multi-supplier inventory model accounted for the unique demand patterns occurring in humanitarian emergency relief operations. Data for the study were collected from their warehouse operations in Lockichoggio, Kenya, responding to the complex humanitarian emergency in south Sudan. Ozbay and Ozguven (2007) worked on a sub-problem of the general humanitarian emergency response problem, namely, an efficient and quick-response humanitarian inventory management model to determine the safety stock while preventing disruptions at a minimal cost. They came up with a realistic model of the time-dependent inventory planning and management problem that could be used for the development of efficient pre and post-disaster plans. The proposed model attempted to determine the minimum safety stock in the emergency inventories so that the consumption of the stocked 
emergency commodities could occur without disruption for a given probability. Ozguven and Ozbay (2009) proposed an efficient multi-commodity solution to enhance and extend the model of Ozbay and Ozguven (2007) using the concept of p-level efficient points (pLEPs). The preliminary results showed that pLEPs solutions were found to be good approximations of exact solution results. Ozguven and Ozbay (2012) applied their model on realistic case studies to examine the differences in emergency inventory strategies for different types of disasters. A similar approach was proposed for fixed lifetime commodities by Jaller et al. (2008) as a cost minimization multi-commodity, multisupplier, two echelon, multi-period stochastic inventory model for disaster planning. They applied their model on a small case study where they found out that the lifetime of commodities should be taken into account while pre-positioning and distributing vital supplies. Mete and Zabinsky (2010) also developed a stochastic programming model for the storage and distribution of medical supplies during the disasters while capturing the disaster specific information and possible effect of disasters through the use of disaster scenarios. With this methodology, they stated that balancing the risk and preparedness was possible in spite of the stochasticities associated with the disasters. They applied the model for possible earthquake scenarios in Seattle, Washington, USA. Recently, BozorgiAmiri et al. (2011) developed a multi-objective stochastic programming approach for disaster relief logistics under uncertainty, with an application on a possible earthquake that could happen in Iran. Their model minimized expected total costs, cost variability, and expected penalty for a possible infeasible solution due to uncertain parameters while maximizing customer satisfaction. However, their model did not have any real time 
scheme that would make sure the planned strategy could work efficiently in the aftermath of a disaster.

Some studies tried to integrate the methodologies for inventory/shelter location and allocation with the distribution of the commodities. Among these studies, Sherali et al. (1991) developed a model that solved the nonlinear mixed-integer programming problem for the selection of shelter locations among potential alternatives in a manner feasible to available resources. The model also gave an evacuation strategy minimizing the total evacuation time with a solution heuristic based on the generalized Benders' decomposition method. Similarly, Yi and Ozdamar (2007) described a locationdistribution model for coordinating logistics support and evacuation operations in disaster response activities. The proposed modeling framework was designed as a flexible dynamic (multi-period) coordination instrument that could adjust to frequent information updates, vehicle re-routing and re-allocation of service capacities. Their aim was maximizing the response service level by enabling fast relief access to affected areas and locating temporary emergency units in appropriate sites. Dessouky et al. (2006) also proposed mathematical formulations on the facility location and vehicle routing problems for the rapid distribution of medical supplies. They applied their model on a hypothetical anthrax emergency in Los Angeles with the help of CPLEX solver where they compared the routes based on the existence of uncertainty. Shen et al. (2009) developed a bi-level model to determine the locations of safety shelters in a transportation network. They proposed two models, the first one addressing the location of safety shelters, and the second one dealing with the decision making during evacuations. Han et al. (2007) also presented a supply location selection and routing problem where any supply source could 
also send a commodity to a sink to take advantage of the multiple supply sources. They tested the model on a small network and found out that the total transportation cost was larger with tight deadlines.

A hierarchical capacitated-median methodology to implement a hierarchical structure among the relief facilities was proposed by Widener and Horner (2010). They used spatial data to place the hurricane relief shelters with different demand, and proposed a method for distributing supplies. They found out that a hierarchical method would help to find the optimal locations of distinct facilities that provide different levels of assistance. As a different approach, Afshar and Haghani (2008) worked on a simulation-optimization framework to find the system optimal solution for an emergency evacuation planning problem. They proposed heuristic algorithms to simultaneously solve for the shelter choice, evacuation route, and departure time for all evacuees from given origins, and worked on a case study from Ocean City, Maryland, USA. Houming et al. (2008) discussed the different characteristics of disaster logistics and general commercial logistics, and focused on the distribution of the vital supplies via the process of dispatching vehicles available at emergency centers to transport those supplies. Their mathematical model aimed to minimize the total distribution time of the emergency vehicles, and they presented a simple example using MATLAB.

Specifically for the flood evacuation plans, Hwang (1999) developed an inventory allocation and vehicle routing model for food supply problems of famine relief. They proposed a 3-step model for item problem, developed a computer program for submodels of food supply in North Korea, and examined this model investigating the sample results. Kongsomsaksakul et al. (2005) also presented a bi-level programming 
methodology for locating and allocating shelters with capacity constraints. They modeled the problem as a Stackelberg game with two players, i.e. planning authority and evacuees. In their model, the planning authority tried to determine the number and locations of shelters with the objective of minimizing the total network evacuation time whereas the evacuees tried to decide which shelter to go by which route given the locations and capacities of shelters.

The introduction of uncertainty into the constraints of the mathematical problems resulted in a need for more challenging methodologies to solve these models. As a result, Yazici and Ozbay (2007) addressed the location and effectiveness of hurricane evacuation shelters with a stochastic programming model including probabilistic constraints. They mainly focused on shelter location and allocation, and capacities of shelters by using a cell transmission model (CTM) for the system optimal (SO) dynamic traffic assignment. The influence of introducing stochastic link capacity constraints in terms of the effectiveness of shelters was analyzed with a simplified version of the complex Cape May (New Jersey, USA) network.

\subsubsection{Use of Information Technologies for Emergency Inventory Management}

Sourirajan et al. (2008) stated that the phenomenon of the variance in the flow of demand information in a multi-echelon supply chain based on uncertain, real-time information (which is present in the aftermath of a disaster) is an example of the need for an online strategy, which can be achieved by information technology (IT). There are several studies that include the use of IT, which covers both computing and communications capabilities to enhance the operational management of natural and 
human-made disasters. Use of IT-based emergency management systems will enhance the disaster management and emergency relief operations in the following ways:

1. Decision and operational support, emergency supply tracking and allocation will be improved using RFID technologies, GIS, online source directories, database systems, and commercial software.

2. Communications will be robust and efficient with the help of cellular systems, wireless/mobile networking and infrastructure, and internet.

3. Infrastructure survivability will be enhanced and the functions of the society will be maintained with renewable power sources, GIS, embedded sensors and detectors for asset evaluation.

4. Emergency response will be faster through the computer-mediated exercises like simulations, online storage for lessons learned, and data collection tools.

5. Engagement of public will be better with the enhanced two-way communication between the public and automated resource contact systems.

National Research Center of the National Academies conducted several studies on the information technologies and their role in emergency management. National Research Center study (2007a) aimed to evaluate the current use of geospatial data and tools in emergency management, and to make recommendations to improve that use. They stated that although geospatial data and tools have evolved over the past four decades into an extremely powerful and effective application of digital technology, and they have been widely deployed in many areas of human activity, emergency management provided a very different context for their application. Their concerns were as follows: 
- Due to the emergency demands occurring under enormous pressure of time rather than weeks or months, data should be acquired and analyzed within minutes.

- Users who rely on the products of geospatial tools have often been poorly trained in their use and have been working under very difficult circumstances. The truth for emergency management systems is that little room exists for error and little admiration for complex technologies that fail under pressure.

National Research Center report (2007b) described both the short- and long-term opportunities to enhance responsiveness and to increase resilience by applying IT to disaster management, and examined the mechanisms to facilitate the development and effective use of IT. They stated that, as in many other sectors, the successful use of IT involved multiple factors:

- Making smarter use of existing technologies.

- Creating opportunities to develop and adopt new technologies.

- Evolving organizational practices to best employ those technologies.

With this idea, they listed these capabilities together with several examples of near-term, mid-term, and long-term opportunities for technology development that were identified by the NRC committee. They argued that different actions were likely to be required to move technologies toward deployment in the field which depended on their place in the technology pipeline, the degree of specialized technological adaptation required for use in disaster management, and the need for organizational changes. In their view, the information technologies have not been nearly as broadly successful in the disaster management due to the following barriers: 
1. Disaster management organizations often lack the resources to acquire valuable capabilities.

2. Both the development and the deployment of many promising technologies are risky and costly compared with the opportunity presented by the commercial market for these technologies today.

3. In most agencies with disaster management responsibilities, there is no one who is charged specifically with tracking IT technology, identifying promising technologies, integrating them into operations, or interacting with IT vendors to make sure that needs are addressed.

4. Decisions regarding IT tend to be made independently by local organizations that must work together in disasters.

5. Disaster management is concerned with environments that are intrinsically uncertain and unstable.

6. Important sources of funds are typically only available once a disaster has been declared and must also be spent in a short window of time.

On June 22-23, 2005, Computer Science and Telecommunications Board (CSTB) of National Research Center Committee on Using Information Technology to Enhance Disaster Management held a workshop in Washington, D.C., to obtain perspectives on the use of IT to enhance the management of natural and human-made disasters. Workshop participants invited as panelists included federal program managers and researchers, state and local officials and first responders, representatives from industry, and academic researchers from a variety of disciplines. In addition to providing perspectives on the current state of the art and practice, workshop presenters also 
described future opportunities to make better use of information technology to improve disaster management. The workshop correspondents agreed on the following issues to improve the use of IT for emergency management:

1. Information technology has a critical and evolving role in disaster management.

2. New research directions for information technology in disaster management should be encouraged.

3. Collaboration, coordination, and interoperability should be pressing issues in a need-to-share world.

Another study conducted by Minyard (2007) stated that evacuation planning relied on the information system that could track people and assets from the planning stage through registration, evacuation, sheltering and re-population phases. According to the study, such a system should depend on wireless communications, untethered field devices, relational databases, GIS, simulation engines, and asset tracking systems linked to RFID tags/readers and bar code scanners. Donahue and Joyce (2001) examined another important dimension of IT-enhanced emergency management, namely the federal disaster funding process, and they provided an example of how application of intergovernmental theory to the realm of emergency management might inform more rational policy design and implementation.

Reviewing the use of technologies for emergency management, Nouali et al (2009) stated that deaths and injuries as well as economic losses could be efficiently reduced by timely and effectively response to the disasters. They presented that the research and development projects through the use of IT in disaster management were basically in the following areas: the use of databases, GIS systems, and wireless and 
mobile technologies. They presented six projects concerned with disaster management, namely:

- $\quad$ DUMBONET (Digital Ubiquitous Mobile Broad-band OLSR-Kanchanasut 2007): It was an emergency network platform developed by AIT Interlab Laboratory, INRIA Institute, and the WIDE project team.

- WISECOM (Wireless Infrastructure over Satellite for Emergency Communications-Berioli 2007): The project, created by the German Aerospace Center and funded by the European Commission, aimed to develop a complete telecommunication solution to rapidly deploy immediate action after a disaster.

- RESCUE (Responding to Crises and Unexpected Events-Mehrotra 2004): The purpose of the project was to radically transform the ability of responding organizations to gather, manage, use and disseminate information both within the emergency response networks and to the general public as well.

- Ad-hoc Infoware (Middleware Services for Information Sharing in Ad-hoc Networks-Sanderson 2007): The project, created by the Research Council of Norway and the Faculty of Mathematics and Natural Sciences at the University of Oslo, implemented a middleware for information sharing in sparse and ad-hoc networks to efficiently use the available infrastructure in a rescue operation.

- Sahana FOSS (Free and Open Source Software-http://www.sahana.lk): This project grew up in Sri Lanka just after the December 2004 Tsunami, and it aimed to develop an integrated set of pluggable, web-based disaster management applications to provide solutions for large-scale humanitarian problems in the relief phase of a disaster. 
- DDT (Tadokoro 2007): This project worked on rescue robots and related technologies for earthquake disaster mitigation in urban areas, and launched by the Japanese Ministry of Education, Culture, Sports, Science and Technology.

Troy et al. (2007) presented the results of a pilot study on implementing a community-based resource database through collaboration with local American Red Cross chapters and public and private community organizations. Here is an example note from the study:

"Imagine that a community volunteer receives requests for shelter that far exceed the bed capacity of a homeless facility. The volunteer consults the community's printed resource guide and refers clients to other amenities. Unfortunately, the printed guide is out of date, and hence the clients have nowhere to go for the night. In this example, a reliable community-based resource for immediately retrieving accurate information is either missing, obsolete, or the critical resources are not listed."

They concluded that a database of community-based resources would enormously help communities to prepare for and respond to emergencies.

Global Disaster Information Network (GDIN) report (1997) provided the potential for reducing disaster costs through better application of information technologies. Their major observations and associated recommendations for better management of disasters were as follows:

1. Availability of disaster information is greater than ever before.

2. Various technical problems appear to be manageable. There are technical barriers, but these appear to be resolvable. 
3. There is a significant need to involve all stakeholders. Disaster response, recovery, mitigation, and preparedness are primarily the responsibility of local communities, although they often require state and Federal assistance.

4. Recognizing that there are many technical and organizational barriers to overcome, it is clear that the information network should be developed using a phased approach.

5. There is a need for clear policies and procedures. Federal Government needs to take direct action in making data and information from classified sources more accessible for disasters.

Based on these studies, it can be noted that, in terms of inventory-related research point of view, one of the most important information technologies is the radio frequency identification devices (RFID). RFID technologies can provide the dynamic change in the inventory levels, flow of emergency vehicles and supplies and even people dead or on the move. Results of a possible RFID application will not only help understanding the pros and cons of the emergency response for the disasters but also it will help the researchers to validate and verify their inventory management and logistics models. RFID literature on emergency management and disaster response is not very wide. One of these few studies include the work of Gadh and Prabhu (2006) who presented some basic information about RFID technologies with supplying the news from Mississippi's Harrison County where U.S. Disaster Mortuary Operational Response Team and health officials implanted RFID chips on Hurricane Katrina victims. They stated that there were several challenges for the implementation of RFID systems: 
1. RFID technology is affected by the presence of metal, liquids, organic matter, and interference from other radio-frequency (RF) sources. These materials cause RF energy absorption, reflection, multi-path, RF signal shading, signal bouncing and skin effects.

2. The adoption of the technology has been hampered by standards, the evolving aspect of which has made industry wary of committing to any particular technology. This made it very difficult to deploy interoperable solutions.

3. Large sections of industry have been waiting for the cost of the RFID technology to come down.

Chatfield et al. (2010) also made an analysis of the literature on existing RFID technology benefits, and concluded that RFID technologies could be used for several basic operations, namely, critical asset tracking and management, location-aware information availability, visibility and information sharing, and decision-making and effectiveness. They also presented the following promising innovative RFID applications:

- SNETS (Special Needs Evacuation Tracking System): This system has been implemented as a part of State of Texas emergency evacuation plan (SNETS, 2008). SNETS was a complete evacuee tracking system which allowed the jurisdiction to match each evacuee with a unique wristband prior to evacuation. Evacuee wristbands were scanned either by RFID or barcode scans at key points in the evacuee's journey so that the jurisdiction could know at any time where evacuees were last "seen" and repatriation could be accomplished more easily.

- Dead Identification: This was performed to be able to track the movement of the dead bodies in transit and their destinations. BBC News (2005) reported that 
Harvard Medical School started to use RFID implant chips injected into an arm of the dead as a means for automatic body identification. News.com (2005) has also stated a similar work where the U.S. Disaster Mortuary Operational Response Team (DMORT) and health officials in Mississippi's Harrison County were implanting human cadavers with RFID chips in an effort to speed up the process of identifying victims and providing information to families.

- Model Validation of Evacuation Simulations: This approach included the usage of RFID tags in person identification during an emergency evacuation. As an example, Hostikka et al. (2007) worked on the video identification and RFID techniques for the fire evacuations. The idea was to find out and compare the real time human evacuation behavior. They stated that promising results were achieved for the use of RFID as a means to make observations on the human movement in the evacuation.

A crucial study on RFID usage for emergency operations was performed by Henderson (2007), who stated that RFID systems could be efficiently used by Battle Command Sustainment Support System (BCS3) to track commodities including emergency supplies such as water, medical materials, and food, in near-real time, flowing in and out of the region. The aim of this study was to provide visibility and information to facilitate the hand-off of commodities and their movement from FEMA to the state and local agencies. To accomplish this mission, Henderson (2007) presented the disaster relief framework and works to be done. The idea was to establish an automation network that had fixed and mobile interrogators to monitor RFID devices online, on a map-based (possibly GIS) computer laptop screen through live tracking tools and in-transit visibility. 
This was attempted as a support provided by the State of Louisiana and the U.S. Army for the Hurricane Katrina aftermath.

There are also a few studies that include an integrated approach of both mathematical methodologies and information technologies. Tomaszewski et al. (2006) designed a Geocollaborative Web Portal (GWP) application to provide a common and intuitive interface through which asynchronous, geocollaborative activities could be conducted in support of humanitarian relief logistics operations. Chang and Hsueh (2007) also developed a flood emergency logistics database for GIS by using the data processing and network analysis functions of GIS, disaster prediction subsystem and rescue resource subsystem. In another study, Iakovou and Douligeris (2001) presented an integrated information management system to serve as a platform that synthesized regularly updated information and decision making models, and De Leeuw et al. (2009) developed a framework of logistics aspects for flood emergency planning. Similarly, Li and Tang (2008) proposed the Artificial Emergency-Logistics-Planning System (AELPS) to help government and disaster relief organizations prepare for and manage severe disasters. This system was based on artificial-society theory, concurrently considering different subsystems dealing with weather, geology, epidemics, and so on. Alcada-Almeida et al. (2009) also worked on a geographical information systems (GIS)-based decision support system with a multi-objective model to locate emergency shelters and to identify evacuation routes. The approach incorporated a multi-objective model into a GIS-based decision support system that planners could access via the Internet. The proposed approach was demonstrated with a case study for the City of Coimbra, Portugal, for the evacuation operations during major fires. Recently, Ozguven and Ozbay (2011) have 
proposed a robust emergency management system with a two step approach, offline planning, and online real time management of emergency management operations. This approach with the application of information technologies (specifically with RFID) would effectively improve the solutions for the stochastic inventory control problem to reflect the real life situations as the needs for people could drastically change during the disaster relief operations, and unexpected major disruptions that cannot be pre-planned could always occur.

\subsubsection{Data Gathering Studies}

As the empirical data required to validate the humanitarian supply chain models is not always readily available, the applicability of the aforementioned theoretical models becomes the weakest link of the literature. Therefore, data gathering studies should be given utmost importance. In collaboration between Rensselaer Polytechnic Institute (RPI) and the University of Delaware, Holguin-Veras et al. (2006-2008) gathered valuable information and data about the basic features of the formal and the informal logistic systems supporting the flows of critical and non-critical supplies to the areas impacted by Hurricane Katrina. The preliminary results of this study were presented by Holguin-Veras et al. (2007, 2008). Holguin-Veras et al. (2007) proposed a synthesis of preliminary suggestions for the improvement of emergency logistics issues. They described these key logistic issues that plagued the response to Hurricane Katrina:

- Magnitude of Requirements,

- Collapse of the Communications Infrastructure,

- Understaffing and Lack of Training, 
- Inefficiencies in Prepositioning Resources,

- Lack of Planning for the Handling and Distribution of Donations,

- Procurement,

- Limited Asset Visibility,

Their suggestions for improvement were:

- Creating a Comprehensive Emergency Logistics Training Program,

- Creating a Robust National Emergency Logistics Network,

- Implementing Measures to Increase Asset Visibility,

- Developing Regional Compacts for Prepositioning of Critical Supplies,

- Implementing Proactive Donation Coordination Plans.

Next, Holguin-Veras et al. (2008) analyzed the requests for critical supplies after Hurricane Katrina by obtaining the temporal distribution of requests indicating the demand and importance of different commodities. The analyses were based on the requests for federal assistance captured in the Action Request Forms (ARFs) issued by responders on site. The data were used to estimate autoregressive integrated moving average (ARIMA) models for the key commodity groups. It was found that the different commodity groups followed structurally different ARIMA processes although the temporal patterns of requests seem similar.

Another data gathering study (Kirsch et al. 2006-continued) was a collaborative PACER project (National Center for the Study of Preparedness and Catastrophic Event Response Project) of Johns Hopkins University, American Red Cross, University of Alabama-Birmingham, Morgan State University, University of South Florida, and Penn State University. The objectives of this study were as follows: 
1. Define and identify the role of informal networks in disaster response.

2. Describe the role of the informal healthcare response to Katrina/Rita.

3. Describe the role of faith based organizations (FBOs) in disaster response.

4. Develop survey tools to assess the informal networks' desire, capacity and needs to respond to a disaster.

Studies related with the experiences of the evacuees located in the disaster shelters also indicate how efficient the relief operations were held in the aftermath of a disaster. After Hurricane Katrina, Brodie et al. (2006) examined the experiences of the evacuees housed in Houston area shelters two weeks after the hurricane. They conducted a survey with 680 randomly selected respondents who were evacuated to Houston from the Gulf Coast as a result of Katrina. The results showed that many evacuees suffered physical and emotional stress during the storm and its aftermath, including living with inadequate food and water. According to this study, $56 \%$ of these Houston shelter residents were left without adequate food, $54 \%$ were left without adequate water, and $32 \%$ didn't receive adequate medical supplies. A summary of the experiences of Houston shelter residents in the immediate aftermath of the storm is given in Table 1. Scurfield (2006) also gave a personal reflective describing the real impact of Hurricane Katrina on the Mississippi Gulf Coast and the surrounding communities. He stated that it was crucial to ensure that basic survival needs of people were being addressed. That is, if people were in need of access to food, water, clothing, bedding, shelter, safety, transportation, financial assistance, they should get them directly. This statement reflected the importance of an efficient inventory control model. Similarly, Webb (2005) described the food and nutrition concerns taken up by the first assessment teams in Aceh after the 
December 2004 tsunami in Northern Sumatra. One of the important findings was that $15 \%$ of people interviewed were eating only one meal per day at the time of interview (almost one month after the shock). Therefore, there was clearly a food and nutrition crisis in Aceh post-tsunami.

Another important issue to consider is the quality of shelters selected for emergency purposes. Nigg et al. (2006) reviewed the disaster literature with respect to how the needs for sheltering and temporary housing developed in the pre-impact and post-impact evacuation situations. They reported that many of the emergency shelters established through the city of New Orleans ended up having to be evacuated since the levees failed.

Realistic analytical models including demand and consumption uncertainties are needed to better plan and manage the inventory of emergency commodities for the major disasters. However, even if the safety stocks for the emergency commodities are planned individually beforehand, the delivery process can be problematic. For instance, Litman (2006) stated that, after the hurricane Katrina, civil organizations were not allowed to transport and deliver the vital supplies to shelters due to the military and safety reasons. In another study, Long and Wood (1995) indicated that the emergency relief operations occurred away from major traffic roadways in less developed regions with inadequate infrastructure. These studies show the unavailability of a sufficient transportation scheme for the emergency commodities to shelters.

A National Institute of Standards and Technology (NIST) study (2011) is also being conducted to determine the impacts of Joplin tornado in Montana, USA. The objectives of this NIST technical study are to determine the characteristics of the wind 
hazard from the tornado; determine the pattern, location and cause of injuries and fatalities, and how these numbers were affected by emergency communications and the public response to those communications; determine the performance of residential, commercial and critical (police stations, firehouses, hospitals, etc.) buildings; determine the performance of lifelines (natural gas, electrical distribution, water, communications, etc.) as they relate to maintaining building operation; and make recommendations, if warranted, for improvements to building codes, standards and practices based on the findings of the study.

There are also several studies directly focusing on people that do not have cars to leave the impact area, people with disabilities, and patients. These studies include the work of Renne et al. (2009) who examined the carless and special needs evacuation planning for five U.S. metropolitan areas. Their discussion was based on stakeholder focus groups across five U.S. regions, including Chicago, Miami, New Orleans, New York, and San Francisco. Childers (2009) similarly developed a decision framework for prioritizing patient evacuations. Rather than observing from the simulation analysis point of view, they observed the system performance under many scenarios and developed some prioritization guidelines that would help determine the need and allocation of transportation resources.

\subsection{Summary}

This chapter focused on the detailed survey and presentation of the available emergency inventory control and management methodologies for disasters, and provided a comprehensive literature review of these methodologies concentrating on the storage, 
location and distribution aspects. It is clear that there are a wide range of inventory related emergency management studies for the relief operations, and that the need for an effective integrated planning and operational capability to address the disaster inventory control problem should be clearly recognized. However, the review reveals that there exists a significant gap between the theory and practice. With this notion, the following future research areas are worth of investigation:

- There is apparently a need for easily understood and applicable models used in providing decision support in the emergency relief operations. Introducing the increased use of implementation aids such as figures and tables, familiarizing the practitioners and planners with the model by interactive tools, and increasing the user-friendliness of the mathematical framework to help the decision making process are some of the methods that can be used for that purpose.

- The most important issue is obviously testing the models using real life data. Not only are the data gathering studies have utmost importance for this purpose but also studies that are improved by data validation and verification will show whether or not the models work properly and efficiently. Hence, data validation and verification have to be performed using the information based on the data gathering studies.

- An important feature of an emergency inventory management framework can be to be able to fit for different disaster scenarios with distinctive characteristics. This will definitely be a critical improvement for the development and implementation of efficient pre and post-disaster plans. 
- Online emergency management is still a very open area to work on. The integration of information technologies and intelligent transportation systems with theoretical models will definitely enhance the inventory management of natural and human-made disasters covering both computing and communications capabilities.

- There are a few studies that can make use of the real-time information regarding inventory levels and supply rates to be able to dynamically adjust inventory management decisions. Therefore, there is a need for realistic and efficient models that integrates the inventory management, real-time monitoring and feedback control models for disasters that incorporate stochastic features of the real life situations.

Based on this information, in the next chapter, we will define our emergency inventory management problem within the disaster framework. 


\section{CHAPTER 3. EMERGENCY INVENTORY MANAGEMENT WITHIN THE DISASTER RELIEF FRAMEWORK}

The 2009 Disaster Reduction Report of the UNISDR (United Nations International Strategy for Disaster Reduction) defines a disaster as follows:

"A serious disruption of the functioning of a community or a society involving widespread human, material, economic or environmental losses and impacts, which exceeds the ability of the affected community or society to cope using its own resources."

These catastrophic events, natural or man-made, are extreme, unexpected events often occurring with little or no warning, and they make all of us acutely aware of our vulnerabilities to disasters. A National Research Council of the National Academies report (2007a) mentioned two important problems faced by unstable disaster situations: the complexity introduced by the dynamics of emergency response operations and information uncertainty due to fluctuating demand and possible disruptions in the transportation network. Unfortunately, the threats posed by these disaster problems are likely to be even worse in the future due to the following reasons:

- Population growth in areas vulnerable to disasters, e.g., along coastal areas or near dangerous faults,

- Production and transportation of toxic and hazardous materials, 
- Rapid growth in terms of technology and industrialization, especially in developing countries.

According to Noji (1997), we will experience many natural hazards during the next decade, specifically about 1 million thunderstorms, a thousand floods, damaging earthquakes, wildfires, hurricanes, tsunamis, droughts, and volcanic eruptions. Table 3.1 shows the distribution of these disasters by type all around the world for the time period 1900-2011 taken from the Emergency Disaster Database (EM-DAT), as provided by the Centre for Research on the Epidemiology of Disasters (CRED). In this database, an event qualifies as a disaster if at least one of the following criteria is fulfilled: ten or more people are reported killed; 100 or more people are reported affected, injured, and/or homeless; there has been a declaration of a state of emergency; or there has been a call for international assistance. Here, affected people indicates the people requiring immediate assistance during a period of emergency, i.e., requiring basic survival needs such as food, water, shelter, sanitation, and immediate medical assistance. As observed, floods and storms (including the effects of hurricanes) are the most common disasters, with the percentages of $32 \%$ and $25 \%$, respectively. Moreover, as seen from Table 3.1, although the numbers of people affected are very high for floods and storms, the numbers of people killed are lower than the numbers for other severe disasters. On the other hand, note that in terms of need for relief, rare disasters such as earthquakes, droughts, fires, volcanic eruptions, infestations, and food shortages require more share than floods and storms. Therefore, these rare disasters require more attention in terms of emergency inventory management. 
Table 3.1 Summary Statistics for Disasters by Type (1900-2011) (http://www.emdat.be)

\begin{tabular}{|c|c|c|c|c|}
\hline \multicolumn{5}{|c|}{ Natural Disasters } \\
\hline $\begin{array}{l}\text { Disaster } \\
\text { Type }\end{array}$ & $\begin{array}{c}\text { Number } \\
\text { of } \\
\text { Disasters }\end{array}$ & $\begin{array}{c}\text { Percentage } \\
\text { Share of } \\
\text { Disasters }\end{array}$ & $\begin{array}{l}\text { Number of } \\
\text { People } \\
\text { Killed by } \\
\text { Disaster }\end{array}$ & $\begin{array}{l}\text { Number of } \\
\text { People } \\
\text { Affected by } \\
\text { Disaster }\end{array}$ \\
\hline Flood & 3883 & $32.21 \%$ & $6,936,146$ & $3,332,422,054$ \\
\hline Storm & 3399 & $28.19 \%$ & $1,379,489$ & $888,852,155$ \\
\hline Epidemics & 1275 & $10.57 \%$ & $10,047,798$ & $50,554,210$ \\
\hline $\begin{array}{c}\text { Earthquake (Including } \\
\text { Tsunami) }\end{array}$ & 1180 & $9.79 \%$ & $2,586,482$ & $169,980,947$ \\
\hline Drought & 603 & $5.00 \%$ & $11,808,692$ & $2,101,143,086$ \\
\hline Mass Movement & 636 & $5.27 \%$ & 60,419 & $13,765,583$ \\
\hline Wild Fires & 357 & $2.96 \%$ & 4,025 & $6,085,821$ \\
\hline Volcano & 221 & $1.83 \%$ & 97,765 & $5,357,731$ \\
\hline Insect Infestation & 85 & $0.70 \%$ & 0 & 553,240 \\
\hline Extreme Temperature & 418 & $3.47 \%$ & 172,189 & $106,660,357$ \\
\hline Total & 12057 & $100 \%$ & $33,098,833$ & $6,675,410,903$ \\
\hline \multicolumn{5}{|c|}{ Technological Disasters } \\
\hline $\begin{array}{l}\text { Disaster } \\
\text { Type }\end{array}$ & $\begin{array}{l}\text { Number } \\
\text { of } \\
\text { Disasters }\end{array}$ & $\begin{array}{c}\text { Percentage } \\
\text { Share of } \\
\text { Disasters }\end{array}$ & $\begin{array}{l}\text { Number of } \\
\text { People } \\
\text { Killed by } \\
\text { Disaster } \\
\end{array}$ & $\begin{array}{l}\text { Number of } \\
\text { People } \\
\text { Affected by } \\
\text { Disaster } \\
\end{array}$ \\
\hline Industrial Accident & 1282 & $17.70 \%$ & 53,238 & $4,891,565$ \\
\hline Transport Accident & 1224 & $16.90 \%$ & 61,323 & $3,451,858$ \\
\hline $\begin{array}{c}\text { Miscellaneous Events (Including } \\
\text { Man-made Attacks) }\end{array}$ & 4738 & $65.41 \%$ & 213,616 & 228,601 \\
\hline Total & 7244 & $100 \%$ & 328,176 & $8,572,023$ \\
\hline
\end{tabular}

Figure 3.1 gives information on the number of people affected and killed for the time period 2004-2005 only for comparison purposes. It clearly indicates that millions of people each year are affected by different disasters, which makes the management of emergency supply inventories a key issue for the sake of victims. It is also clear that the vital needs of disaster victims should be supplied efficiently throughout the entire preand post-disaster period. Without an adequate stock and efficient supply of vital 
commodities, satisfying the daily requirements of disaster victims without disruptions might be problematic.

Human impact by disaster types:

comparison 2004-2005

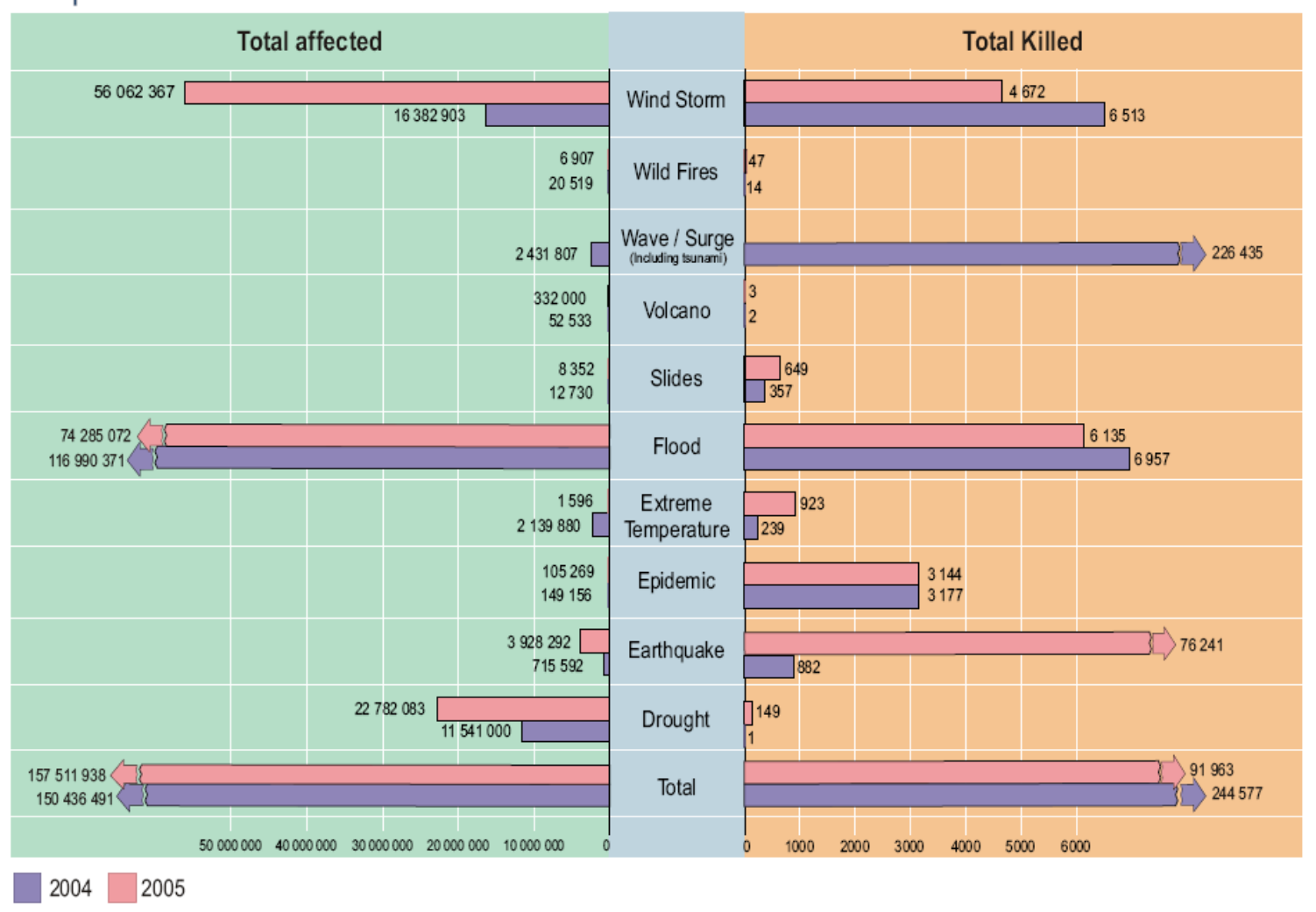

Figure 3.1 Disaster Impact on People: Comparison for 2004 and 2005 (http://www.emdat.be)

In this dissertation, we focus on the basic survival needs of these affected people. Consider a disaster affecting a region. Depending on the strength or severity of the disaster, people may be gathered into shelters to ensure their security (see Figure 3.2). Many of these people, as in the case of Hurricane Katrina, might not have financial resources to leave the disaster area and to find food, drugs, clothes, or other basic needs. Others might want to stay close to their homes and might not want to travel too far, 
mainly due to a desire to go back to their homes once the threat of the specific disaster is over. Therefore, apart from disasters that have low strength, shelters are definitely needed for satisfying the nutritional, physical, and medical needs of survivors. In these shelters, the needs of these evacuees should be supplied efficiently throughout the entire pre- and post-disaster period (see Figure 3.3).

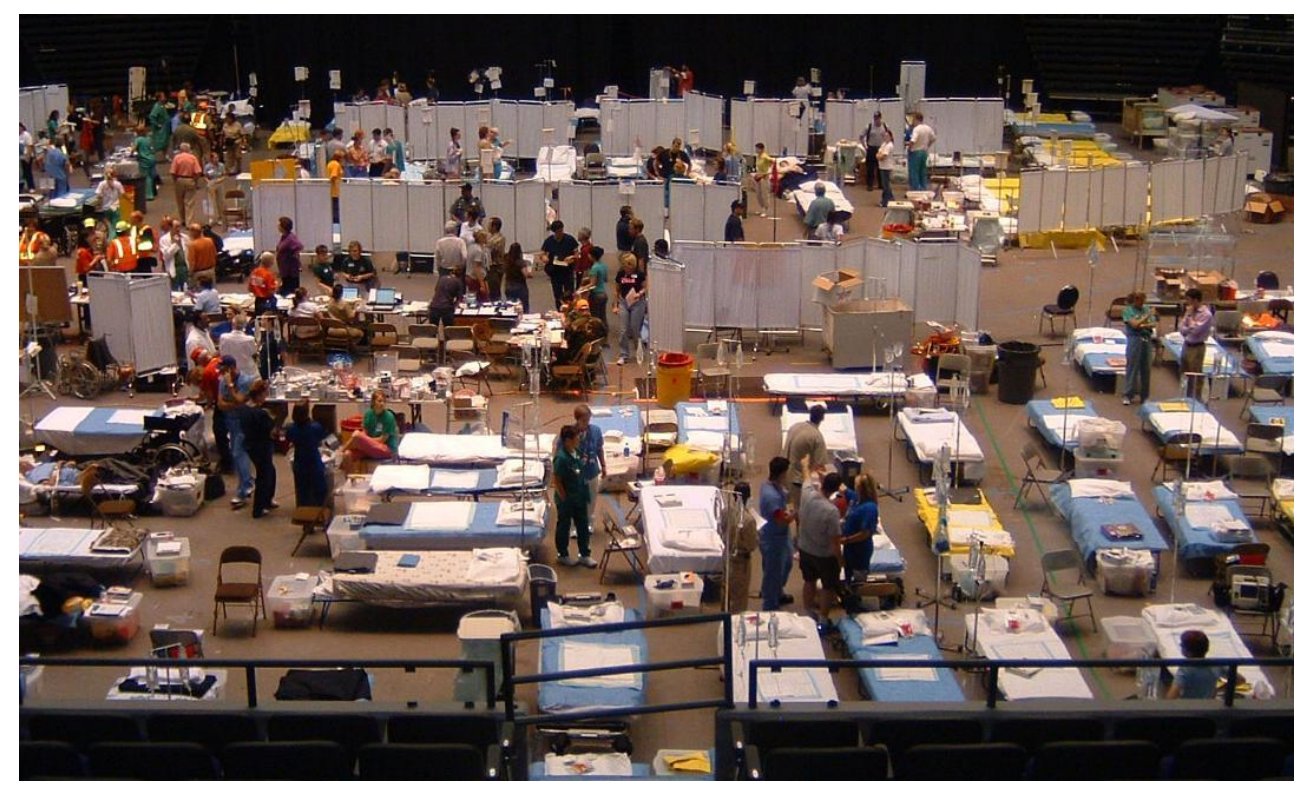

Figure 3.2 A Disaster Shelter (http://www.jhsph.edu/bin/h/e/beds.JPG)

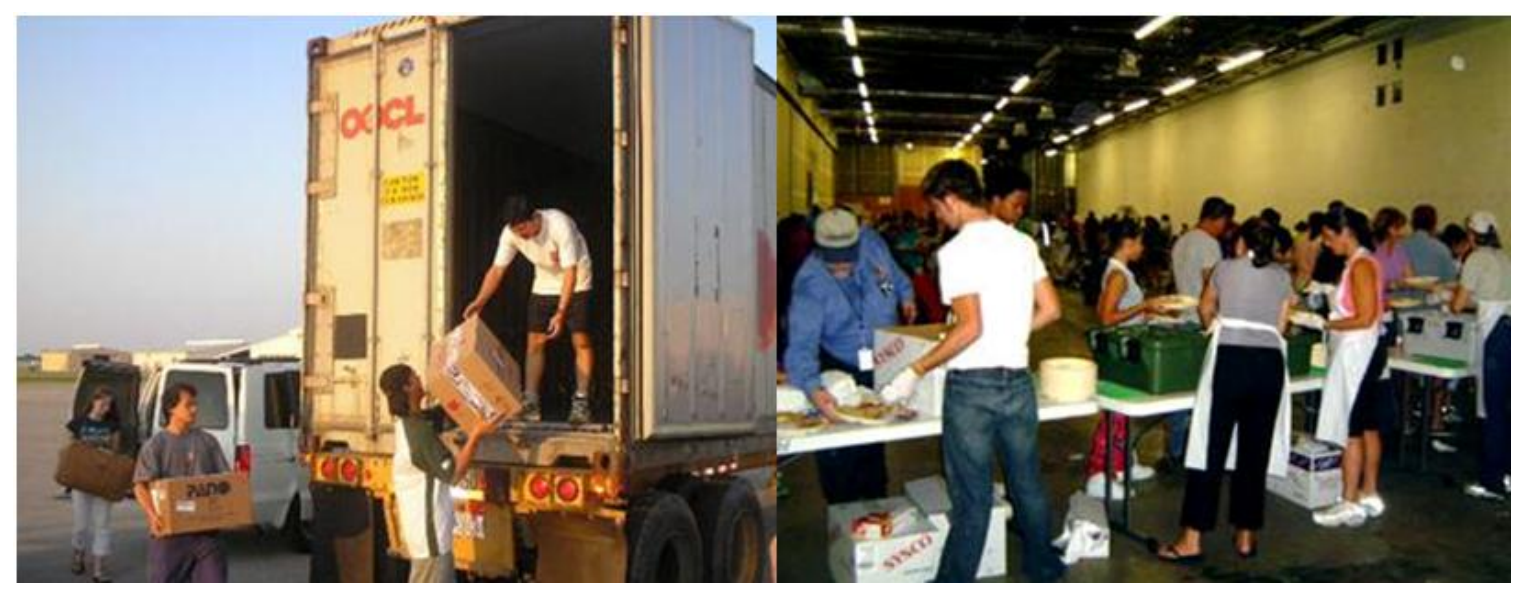

Figure 3.3 Supply of Basic Needs for Disaster Victims (http://www.disasterreliefteam.org) 
Moreover, in this research, we are mainly focused on the following disasters that affect the lives of people substantially (although the method is applicable to many lowimpact extreme events as well). These disasters can be divided into four main categories:

- Dispersed and sudden onset disasters: This type of disaster spreads over and therefore affects a large region and happens very fast, such as the 2004 Indian tsunami.

- Dispersed and slow onset disasters: This type of disaster also spreads over and affects a large region, but it covers a longer time period than sudden disasters. Examples include the 2009 avian flu epidemic and the famines in Africa.

- Localized and slow onset disasters: This type of disaster affects a specific location rather than a large region, and it takes a long time period, as the severity of the disaster increases gradually; an example is the infamous 2005 Hurricane Katrina.

- Localized and sudden onset disasters: This type of disaster also affects a specific location rather than a large region, but it happens quickly, as in the case of 2010 Haiti earthquake and man-made disasters.

The difficulty in handling emergency operations and disaster relief response differs for each type of disaster, as seen in Figure 3.4. To account for that difficulty, an efficient inventory management system should be capable of handling different types of disasters with different characteristics (localized or dispersed, slow or fast). 


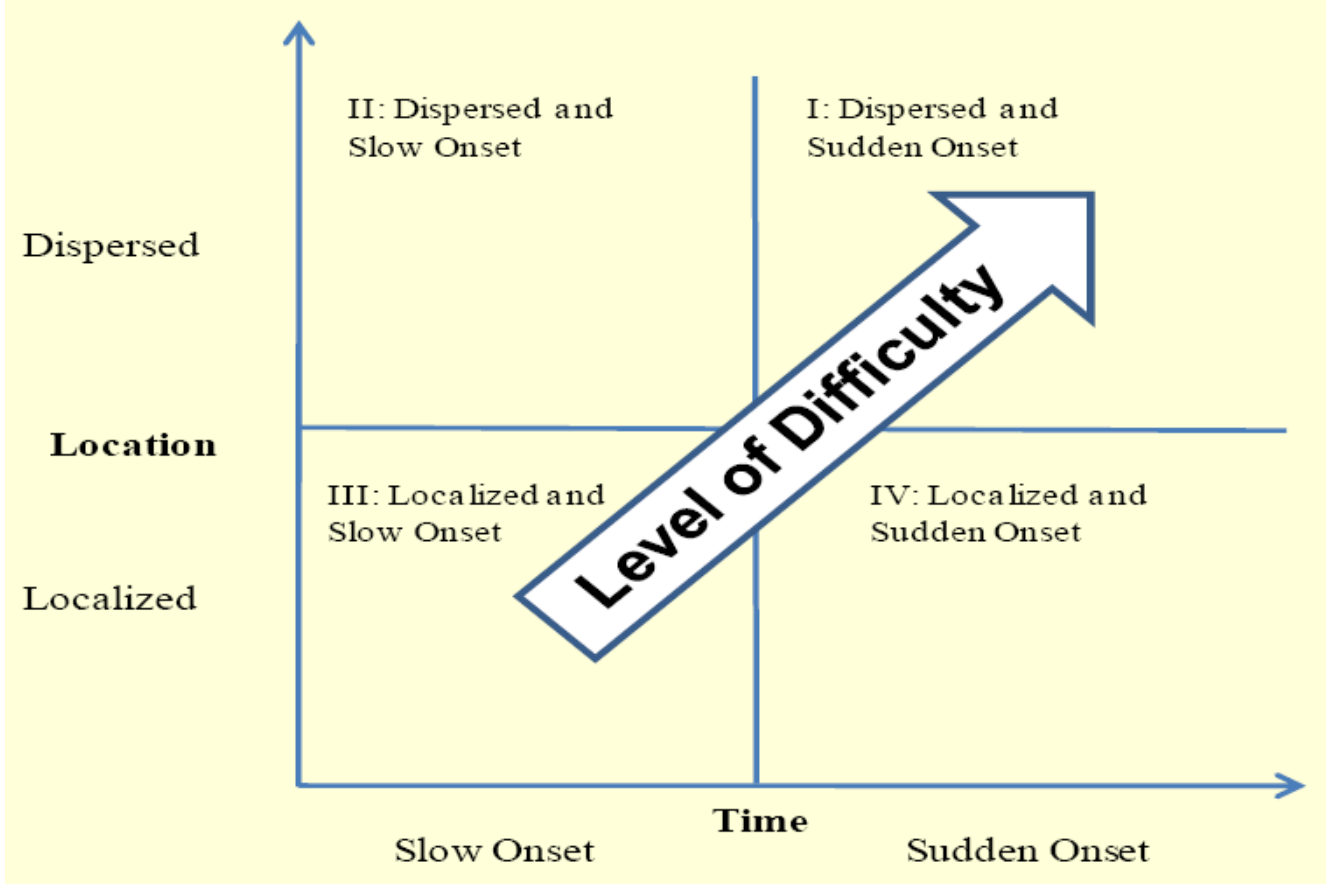

Figure 3.4 Classification of Disasters (Apte and Yoho, 2011)

In this dissertation, different inventory strategies for these distinct types of disasters will be obtained to be able to efficiently respond to each one of them. Regarding this essential information about the characteristics of the disasters, we will define and study the emergency inventory management problem within a disaster framework in this chapter. First of all, there are a number of objectives that should be sought by planners, researchers, and engineers to obtain an efficient emergency inventory management methodology. These objectives mainly differ from commercial inventory management, as disaster inventory management is unique and significantly different from the classical inventory and manufacturing framework. In our emergency management problem, we include the following objectives:

- Minimization of suffering and maximization of chance of survival for victims. 
- Maximization of the available vital supplies for victims.

- Ensuring the flexibility of inventory operations in the presence of uncertain demand and supply and a dynamically changing environment.

- Cost minimization (storage, shortage, surplus, etc.).

The constraints for the emergency inventory management problem, on the other hand, include:

- Storage space constraints,

- The minimum tolerable disruption level (possibly due to transportation, extreme demand, and supplier-related disruptions) constraints.

Figure 3.5 illustrates the overall problem with the objective function and its constraints within the disaster management concept.

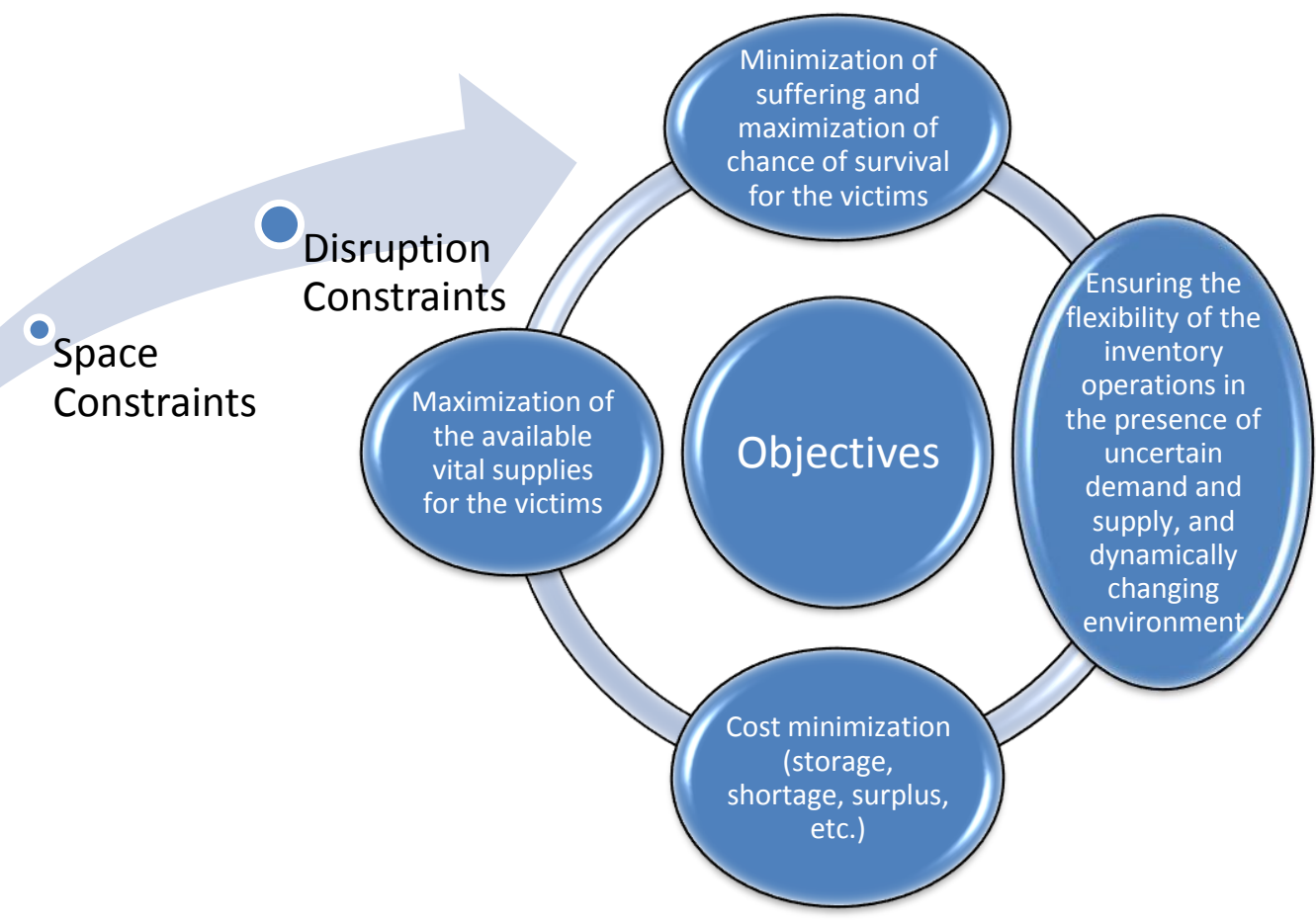

Figure 3.5 Overview of the Emergency Inventory Management Problem 
With these objectives and constraints, the three key questions that an emergency inventory management model should attempt to answer are:

- Can sick or hurt people have a chance to receive the medical care they need?

- Can people find enough survival needs (food, water, clothing, etc.) and adequate shelter so that they can survive for a relatively extended period of time after a disaster?

- Can the transportation system in the impact area be sufficient for the distribution of vital supplies?

Based on these key issues, the basic control concept of our emergency inventory management system, with the hierarchy of decisions, is illustrated in Figure 3.6.

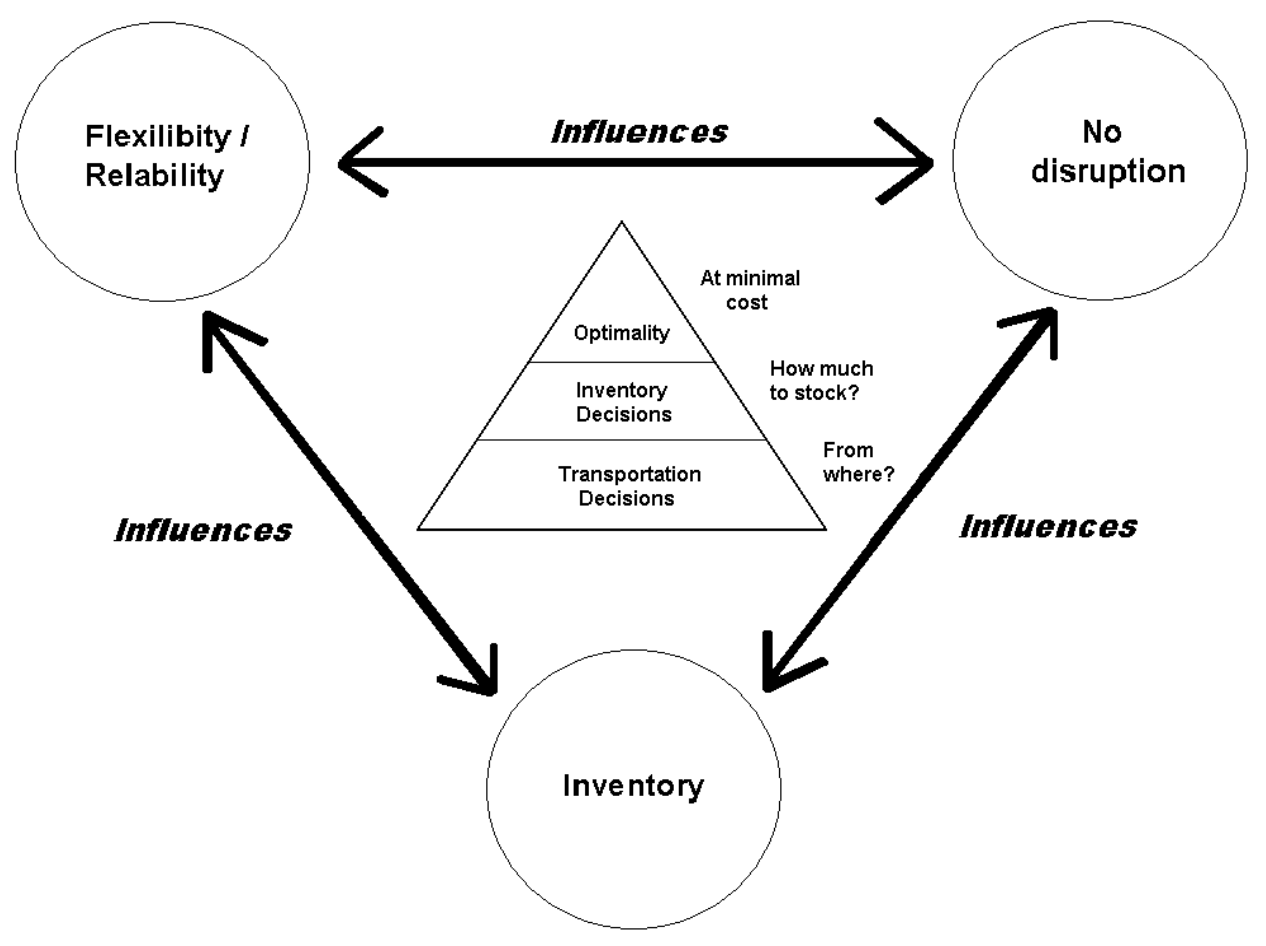

Figure 3.6 Basic Control and Hierarchy of Decisions 
This emergency inventory management and control concept raises several issues to be considered for emergency disaster inventories. The first includes decisions on inventory control, storage, and allocations, as the needs of survivors can drastically change during disaster relief operations. Optimally stocked inventories in pre-planned stock arrangements will increase the efficiency of disaster relief operations. Secondly, the transportation system might not be able to sustain the flow of emergency commodities to the shelters, mainly due to increased demand, reduced supply, and possible transportation system disruptions. As mentioned before, on August 28, 2005, the Louisiana National Guard delivered seven truckloads of MRE's to the largest shelter in New Orleans, the Superdome, enough to supply 15,000 people for three days. This was not enough for the 20,000 victims located in the Superdome. This indicates that during the recovery period after a disaster, emergency, rescue, and commodity supply activities should take place even if the transportation network is heavily damaged or degraded.

An efficient example of how to cope with disruptions when proper emergency inventory management techniques were used was Wal-Mart's performance after the Hurricane Katrina disaster in the Gulf coast in August, 2005 (Leonard, 2005). Wal-Mart employed personnel dedicated to planning for and tracking potential disruptions. With Katrina approaching, Wal-Mart overstocked its nearby distribution centers with commodities that would be needed the most such as bottled water and generators, and after Katrina struck, Wal-Mart's efficient inventory management and delivery system allowed it to respond quickly to deliver supplies and end the disruption to its supply chain. Without this inventory management planning, Wal-Mart's recovery time would have been much longer and much more costly both for the company and the public. 
In this thesis, a two-step approach is proposed to have such a robust emergency inventory management system that can effectively satisfy the needs of victims by solving the aforementioned problems associated with inventory management and supply distribution:

- Offline planning, and

- Online real-time management of emergency inventory management operations.

Figure 3.7 indicates the proposed integrated strategy as a real-time online feedback control scheme where the use of offline and online models is clearly illustrated. This online production policy is necessary due to the expectations of fluctuating demand for vital supplies such as food and medicine during the disaster relief period in shelters. Even if the planning strategy is working without any disruption after the disaster, unexpected problems can always occur. Therefore, an emergency inventory management system should include an online operational policy to minimize the impacts of these unforeseen disruptions, or at least to address the problem at hand as quickly as possible.

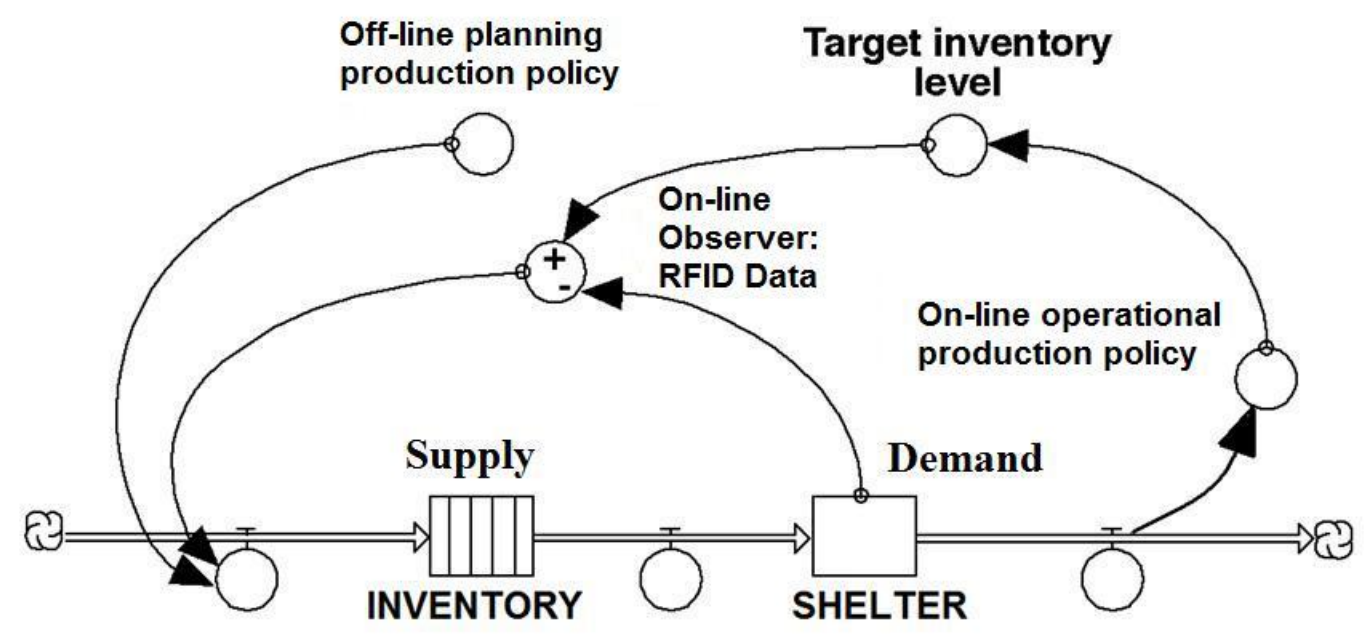

Figure 3.7 Comprehensive Feedback-based Emergency Inventory Control Strategy 


\subsection{Motivation and Intuitive Explanation}

Our mathematical model is developed to cope with disruptions that can occur during emergency relief operations. Intuitively, these disruptions can occur due to a damage in the transportation network, unexpected fluctuations in demand, lost inventories, and unavailability of suppliers or emergency vehicles. The important point is that emergency officials should have a way to anticipate, or at least to respond to these disruptions with careful planning and efficient pre-stocking of vital supplies.

Within the mathematical models proposed in this thesis, consumption (demand) and supply (delivery) distributions are assumed to be continuous distributions. They can be obtained from real-life data collected from past disasters, if possible. In the absence of such data for the case studies presented for Hurricane Katrina in this thesis, supplies and demands are based on the assumptions given in Lee et al. (2009) and the vital supplies delivered during the emergency operations conducted by the Louisiana National Guard.

In our model, disruptions in the transportation network are represented by the change in the number of deliveries that reach the emergency shelters from the inventories. Sensitivity analyses are conducted to observe the behavior of the model with respect to different number of deliveries, and therefore different levels of disruption. Disruption in the supply and demand is represented by a disruption probability that is employed within a probabilistic constraint in the model, where the objective of the constraint is to have initial safety stock and deliveries greater than the demand with a given probability level. 
Initial safety stocks for vital supplies are calculated optimally as part of disaster planning. However, there is definitely a need to maintain optimal inventory levels in the aftermath of a disaster that can rapidly change due to possible disruptions in the transportation and infrastructure system, and due to random and highly dynamic changes in evacuee demand and available resources. Therefore, in this thesis, offline planning and production policies are integrated with an online inventory management strategy to keep track of demand fluctuations for vital supplies (food, water, medical supplies, etc.) as well as transportation network disruptions during the disaster relief period. To be able to validate and verify such an approach within the emergency inventory management framework, there is also a need for real-time tracking of vital supplies being delivered to the shelters. For this purpose, emerging ITS technologies such as Radio Frequency Identification Devices (RFID) can be effectively used for online commodity tracking and logistics and therefore will be discussed in the next section before the introduction of the theoretical model.

\subsection{Possible Application with Radio Frequency Identification Devices (RFID)}

It is very important to understand that the high level of uncertainty during humanitarian relief operations makes resource tracking in the aftermath of these events a crucial requirement. With continuous position reporting through the possible use of Global Positioning Systems (GPS) and satellite connections, RFID technologies can provide dynamic changes in inventory levels and the flow of emergency vehicles and supplies. RFID is a non-line-of-sight (capable of communicating remotely even when 
obscured) and contactless (without direct contact between the transacting elements) automatic identification technology. The identification data is stored on chips that can be attached or embedded into products, animals, and even humans. The ID or user data is written and retrieved to/from the tag by a reader using the energy generated by induction or radiation (Gadh and Prabhu, 2006). However, the important thing is that the RFID tag can be active (with onboard power source) or passive (with no power source). Passive tags have a long life, but they work with relatively short range of activity. On the other hand, active tags have an effective operating range, but the battery shortens the life span. For the emergency relief operations, active tags may be preferable due to their long range, however the maintenance of the tags should be performed with routine checks to ensure that the batteries are properly working.

Data obtained from RFID can be fed into the planning model to obtain realistic delivery and consumption distributions, which can be subsequently used during online feedback control. Within this method, the results of a possible RFID application will not only help in understanding the pros and cons of the emergency response for disasters, but also help to validate and verify our model. Therefore, before introducing the offline and online methodologies in the following chapters, we will first study the logistics aspects of RFID devices in detail.

Theoretically, if historical data become available to obtain the real response of the modeled emergency system, then it will be possible to use standard optimization algorithms and therefore to obtain a controller that can efficiently create historical inputs, controls, and outputs. Thus, as an approach for real-world conditions, it is possible to use RFID systems to track the in and out flow of emergency supplies. An example 
application of RFID focusing on the logistics and transportation aspects of the disaster management problem can be seen in Figure 3.8.

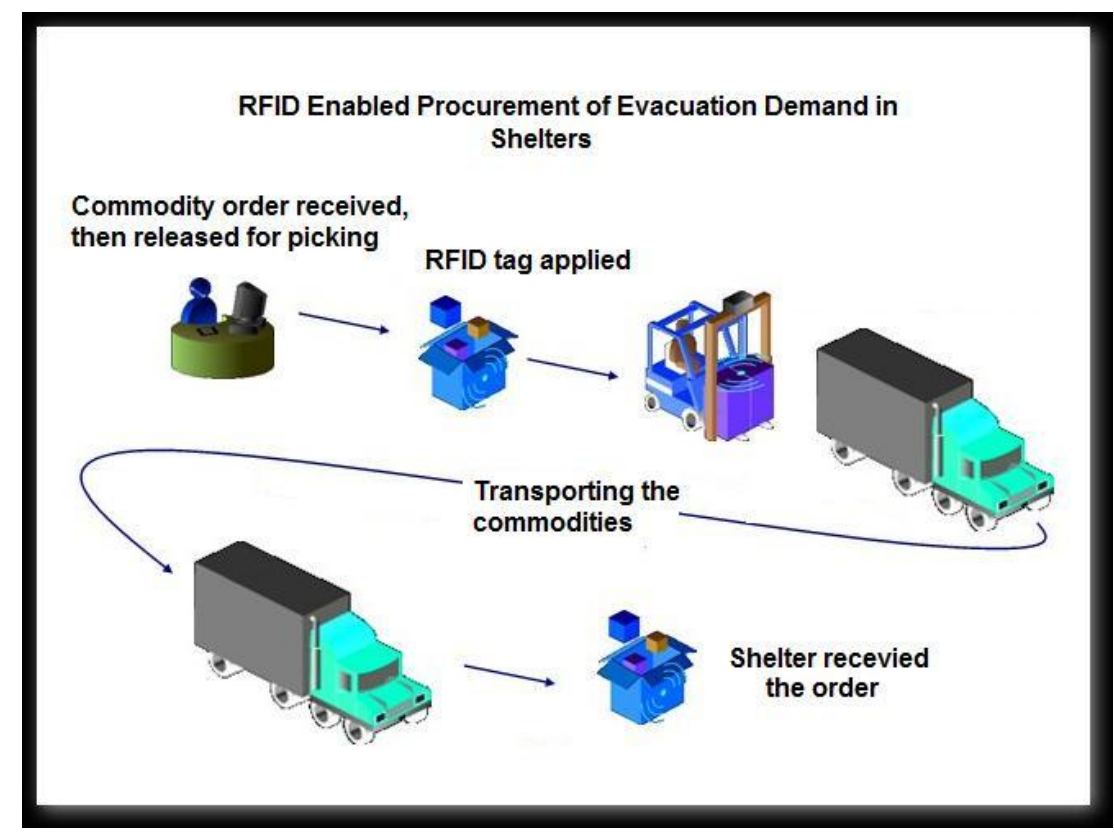

Figure 3.8 RFID Enabled Procurement of Evacuee Demand in Shelters

Basically, RFID technologies have three main advantages for emergency operations:

1. Tracking: This function of RFID works to identify the motion of any emergency supply. The idea includes not only real-time position tracking (flow of commodities), but also tracking motion through entry and exit points.

2. Identification, sensing, and authentication: This can be used for identifying the victims of the disaster to have accurate information. Most importantly, this will help to accurately respond to the needs of patients in the disaster area. It can also be used during the process of tracking commodities such as medicine and blood to enable secure medical assistance to victims. 
3. Automatic data collection and transfer: This is mainly used for minimizing data entry and collection errors and reducing information processing time and inventory management problems.

On the other hand, a thorough literature review on RFID systems (see the Literature Review chapter for more details) revealed the following barriers and obstacles for the implementation phase:

1. Interferences/Reliability/Environment: Disaster management is concerned with environments that are intrinsically uncertain and unstable. Therefore, a lack of standardization of the RFID protocols at the hardware and software levels, and a lack of battery power (presence of power outage) for active RFID tags may cause severe problems. Moreover, RFID technology is affected by the presence of metal, liquids, and organic matter, as well as interference from other radiofrequency $(\mathrm{RF})$ sources. These materials cause RF energy absorption, reflection, multi-path, RF signal shading, signal bouncing, and skin effects.

2. New Technology Problems: The adoption of the technology has been hampered by standards. This makes it very difficult to deploy interoperable solutions. Both the development and deployment of many promising technologies such as RFID systems are risky and costly compared with the opportunity presented by the commercial market for these technologies today.

3. Costs: The industry has been waiting for the cost of RFID technology to come down. Moreover, important sources of funds are typically only available once a disaster has been declared and must also be spent in a short window of time. 
4. Operational/Capacity/Managerial/Knowledge issues: Disaster management organizations often lack the resources to acquire valuable capabilities. In most agencies with disaster management responsibilities, there is no one charged specifically with tracking RFID technology, identifying promising technologies, integrating them into operations, and interacting with RFID vendors.

5. Cultural and ethical concerns: In the case of RFID chips being used on cadavers or victims, there are many oppositions due to cultural, religious, social, and ethical concerns.

6. Privacy, security, data integrity, and legal issues: RFID systems used for emergency management require special security and privacy in order to maintain the feasibility of the flow of goods and people, and to avoid the misuse of the data. The application of the technology is always bound to legal requirements that should be fulfilled, and no personal and confidential information should be transmitted via tags.

7. Local issues: Decisions regarding RFID should be made jointly by local municipalities and organizations that must work together during disasters, which is most of the time one of the most important drawbacks in transportation-related work.

Based on careful study of the literature on RFID systems, this study indicates the following ways to overcome the aforementioned drawbacks:

1. Researchers are working on new RFID technologies such as software-defined radio based devices (Islam et al., 2009). This strategy allows innovations in the physical layer of the RFID that will minimize the effects of most of the challenges 
listed in Interferences/Reliability/Environment. Moreover, the passive RFID tags can be read if passed within close enough proximity to an RFID reader even without a battery, which can solve the power outage problem.

2. Operational/capacity/managerial problems should be solved by establishing control (mobile and permanent) centers all over the region. In this way, it is possible to maintain efficient management and tracking of emergency commodities. This will allow officials and planners to make sure that the basic survival needs of victims are effectively satisfied. It will also be possible to provide the capability to see and track emergency vehicles as they move throughout the disaster region. This will clearly help to determine the degradation of the transportation network, as some of these vehicles may need alternative routing to reach their destinations.

3. Educating officials and personnel is a must to efficiently respond to inventory management and transportation problems during the aftermath of a disaster. New technology- and knowledge-related problems can be solved with the help of private companies that are familiar with the technology, which can help in the training of personnel.

4. The goal of federal and state agencies, the U.S. Army, and private companies should be to create a force structure to meet the severe situations (insufficient commodity supply, disruptions in the roadway network) that can occur after a disaster. This force should be the key to solving local problems; cultural, ethical and religious concerns; and privacy, data integrity, and legal issues. Mostly, as RFID systems are vulnerable to compromise and tags can be removed easily, a 
legal force with the support of an army would be a necessity during or after a disaster.

5. Cost and implementation problems can be handled by a cost-justified incorporation of federal/state agencies as well as private companies such as RFID vendors.

Based on this detailed information on the pros and cons of RFID technologies, we will present a framework for implementing RFID within emergency inventory management operations in the next subsection.

\subsubsection{Implementation using Our Integrated Emergency Management Model}

Henderson (2007) stated that RFID systems could be efficiently used by the U.S.

Army Battle Command Sustainment Support System (BCS3) to track commodities including emergency supplies, in near-real time, flowing in and out of the region. The idea was to establish an automation network that has fixed and mobile interrogators to monitor RFID devices online on a map-based computer screen through live tracking tools and in-transit visibility. With this motivation, we define our RFID-based emergency management and relief system as the process of synchronizing the critical supply and transportation network to facilitate sustainable disaster tracking and to provide an efficient distribution of flow in support of the four stages of disaster relief efforts defined by Henderson (2007). These stages are revised and adapted to our emergency management system based on a time framework (Figure 3.9). 

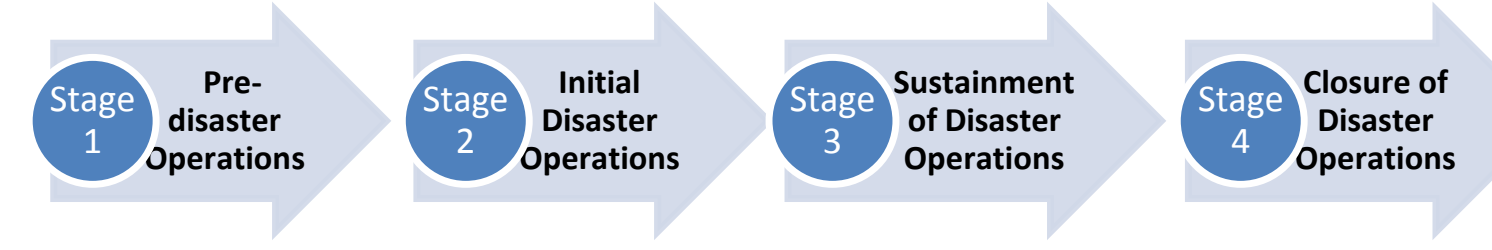

Figure 3.9 Four Stages of RFID-based Emergency Management System for Disasters (Adapted from Henderson, 2007)

These emergency relief framework stages are defined as follows:

1. Stage 1 (Pre-disaster Operations): This is probably one of the most important stages of the whole process. It begins by establishing a link among different levels of government, local organizations, the military, civilian agencies, and the private sector. This will help to construct a workable and executable support plan followed by identifying and notifying available assets in the disaster region. Moreover, tests and exercises should be conducted to validate and verify the implementation of the plan.

2. Stage 2 (Initial Disaster Operations): As coordination among agencies is obtained in the previous stage, Stage 2 makes use of this in terms of preparedness for the delivery and consumption processes of the emergency supplies. This requires the identification of possible distributors and inventories for use, the estimation of the emergency commodities stocked in these inventories, and the establishment of methodologies to assist personnel working in commodity flow management. The proposed offline planning model, therefore, will be extremely useful in this stage, as it will enable us to determine the initial safety stocks in the inventories. 
3. Stage 3 (Sustainment of Disaster Operations): This is basically the online emergency management step where the officials manage commodity flow and distribute commodities across shelters. Therefore, this stage requires the careful prediction of the safety stocks and workforce and the continuous capability of intransit visibility of the emergency commodities within the transportation network. This can be provided by an RFID implementation within the system to forecast and report the requirements of the victims in the shelters, and to support forward demands. Our proposed online model should make use of the RFID data in this stage.

4. Stage 4 (Closure of Disaster Operations): This stage basically ends relief support procedures like the management of vital supply flow and personnel. The data collected and lessons learned should be provided for future reference.

With the definition of these stages, we have introduced the possible use of RFID systems within our framework. However, in the absence of such data, the data for the online inventory control models will be generated by the multi-commodity SHIC model using the pre-defined probabilistic consumption and delivery distributions of emergency commodities.

In the following chapters, we will extensively study the offline and online methodologies proposed within our emergency inventory management framework. 


\section{CHAPTER 4. OFFLINE PLANNING: STOCHASTIC HUMANITARIAN INVENTORY CONTROL MODEL}

The mathematical modeling of disaster inventory control systems has recently been receiving increasing attention in the literature, as efficient and quick-response inventory management has utmost importance during emergency operations. However, there is still a growing need for more general and realistic models that incorporate the uncertainties for the demand and consumption processes in the aftermath of a disaster and that are applicable to any disaster type via changing the relevant parameters. This chapter focuses on obtaining an effective planning methodology, namely the stochastic humanitarian inventory control (SHIC) model, which was first proposed by Ozbay and Ozguven (2007) based on the Hungarian Inventory Control Model introduced by Prékopa (2006).

Several advantages of the proposed model can be summarized as follows:

- The proposed mathematical programming model allows the simultaneous and continuous consumption and delivery of emergency supplies without disruption.

- Delivery and consumption are modeled as stochastic processes due to the high levels of uncertainty associated with extreme events such as hurricanes, earthquakes, or terrorist attacks. 
- The problem can be solved for a range of demand values, allowing one to observe the changes in safety stock values for different consumption amounts. This self-controlling mechanism gives analysts the chance to determine safety stock values more accurately.

The following sections provide a mathematical description of the SHIC model in the context of humanitarian logistics (Ozbay and Ozguven, 2007).

\subsection{Basic Assumptions}

The "stochastic humanitarian disaster inventory control" (SHIC) problem, as defined in this study, is to find the amount of safety stock in the disaster inventories with a probability $1-\varepsilon$, so that independent delivery and consumption processes go on without disruption at minimum cost. For instance, if the value of $\varepsilon$, the probability of disruption, is 0.1 , the aim is that disruption will not occur $90 \%$ of the time.

The following modeling assumptions are made for the finite time interval $[0, T]$.

- In our model, we assume that deliveries, fixed and designated by $n$, take place according to some random process at discrete times within a finite time interval $[0, T]$

- Deliveries, fixed and designated by $n$, take place at discrete times, which can be obtained from past history. These random times have joint probability distributions the same as that of $n$ random points chosen independently from the interval $[\tau, \tau+T]$ according to a uniform distribution.

- The delivery and consumption processes are stochastically independent. 
- A minimal amount, $\delta$, is delivered with each delivery $n$. If the total amount of delivery is $D_{u}$ for some support $u, u \in U, U$ being a finite set of supports, there is also a random amount of delivery obtained by choosing a random sample of size $n-1$ from a population uniformly distributed in the interval $[0,1-n \delta]$.

- Deliveries of emergency order quantities are not based on an extensive transportation network analysis. We assume that a planned delivery is not possible when the transportation network is disrupted. Therefore, disruptions in the transportation network are represented by a decrease in the number of deliveries.

- The consumption process is defined similar to the delivery process, with parameters $C_{u}$ for some $u, u \in U$, as the total amount of consumption, $\gamma$ as the minimal amount of consumption, and $s$ as the number of consumption times.

- We assume that backlogging (ordering a commodity and receiving it after a lead time) is not allowed within our emergency inventory management model.

- We approximate the joint distribution of the random consumption and delivery variables using an approximate multivariate normal distribution with the random variable $W_{i u}^{(l)}$ for each commodity $l=1, \ldots, r$, for $i=1, \ldots, n$, and for each support $u, u \in U$

- To maintain the convexity of the overall nonlinear programming problem, we assume and choose convex cost functions in the objective function. 


\subsection{Methodology}

We will now mathematically describe the model in the context of humanitarian logistics. Note that we assume that delivery and consumption processes are independent, and there will be a superscript $l$ for each commodity.

In each time interval, a minimal amount equal to $\delta \geq 0$ is delivered. Then, a sample size of $L(L \geq n)$ is taken from the uniformly distributed population in the interval $\left[0, D_{u}-n \delta\right]$ and given by $x_{1}^{*} \leq x_{2}^{*} \leq \ldots \leq x_{n}^{*}$. Then, $n-1$ positive integers are taken as $j_{1}<j_{2}<\ldots<j_{n-1} \leq L$. The delivered quantities of the emergency commodity in the $n$ time intervals are assumed to be as follows:

$$
\delta+x_{j_{1}}^{*}, \delta+x_{j_{2}}^{*}-x_{j_{1}}^{*}, \ldots, \delta+x_{j_{n-1}}^{*}-x_{j_{n-2}}^{*}, \delta+\left(D_{u}-n \delta\right)-x_{j_{n-1}}^{*}
$$

The model for consumption is similar. A sample size of $N$ is taken from the population uniformly distributed in the interval $\left[0, C_{u}-n \delta\right]$ and designated by $y_{1}^{*} \leq y_{2}^{*} \leq \ldots \leq y_{n}^{*}$. Then, $n-1$ positive integers are taken as $k_{1}<k_{2}<\ldots<k_{n-1} \leq N$. The consumed quantities are assumed to be as follows:

$$
\gamma+y_{k_{1}}^{*}, \gamma+y_{k_{2}}^{*}-y_{k_{1}}^{*}, \ldots, \gamma+y_{k_{n-1}}^{*}-y_{k_{n-2}}^{*}, \gamma+\left(C_{u}-n \gamma\right)-y_{k_{n-1}}^{*}
$$

Assuming that the delivery and consumption processes are independent, we let

$$
\begin{gathered}
X_{1}=x_{j_{1}}^{*}, X_{1}=x_{j_{2}}^{*}-x_{j_{1}}^{*}, \ldots, X_{n-1}=x_{j_{n-2}}^{*}-x_{j_{n-1}}^{*}, X_{n}=\left(D_{u}-n \delta\right)-x_{j_{n-1}}^{*} \\
Y_{1}=y_{k_{1}}^{*}, Y_{1}=y_{k_{2}}^{*}-y_{k_{1}}^{*}, \ldots, Y_{n-1}=y_{k_{n-2}}^{*}-y_{k_{n-1}}^{*}, Y_{n}=\left(C_{u}-n \gamma\right)-y_{k_{n-1}}^{*}
\end{gathered}
$$


Let $S_{u}$ denote the initial safety stock of commodities for some $u, u \in U$. Another assumption is that we always want to have a safety stock, shown as $S_{u}+D_{u} \geq C_{u}$. Then, the condition of no disruption for each $u, u \in U$ is formulated as follows:

$$
\begin{gathered}
S_{u}+\delta+X_{1} \geq \gamma+Y_{1} \\
S_{u}+2 \delta+X_{1}+X_{2} \geq 2 \gamma+Y_{1}+Y_{2} \\
\vdots \\
S_{u}+(n-1) \delta+X_{1}+X_{2}+\ldots+X_{n-1} \geq(n-1) \gamma+Y_{1}+Y_{2}+\ldots+Y_{n-1} \\
S_{u}+n \delta+X_{1}+X_{2}+\ldots+X_{n} \geq n \gamma+Y_{1}+Y_{2}+\ldots+Y_{n}
\end{gathered}
$$

The inequality $S_{u}+D_{u} \geq C_{u}$ can also be shown in Equation (4.4). However, it is removed as it is aimed to have a model without disruption.

The problem has two stages; hence, there are first- and second-stage decision variables: a subscript $u$ is given to each second-stage variable. The first-stage decision variables in the model are $M^{(l)}$, the storage capacity of each commodity $l=1, \ldots, r$. The second-stage variables are $m_{u}^{(l)} \geq 0$, the additional safety stock for each commodity $l=1, \ldots, r$, and for each $u, u \in U$. We have an initial safety stock in the interval $[0, T]$, and to satisfy the needs of the victims located in the shelters in terms of the commodities, we are trying to find the optimum additional safety stocks, $m_{u}^{(l)^{*}}$ values, and the optimum storage capacities $M^{(l)^{*}}$. The convex cost functions corresponding to the storage capacities $M^{(l)}, l=1, \ldots, r$ are $g^{(l)}(x), l=1, \ldots, r$. The second-stage problem comes up after the delivery values $D_{u}=\left(D_{u}^{(1)}, \ldots, D_{u}^{(r)}\right)$ are observed. The corresponding probabilities for each $\left\{D_{u}, C_{u}, u \in U\right\}$ are given by $p_{u}$. This approach allows us to calculate the total cost where the highest and lowest values have the lowest probabilities 
according to a pre-determined discretized normal distribution. We prescribe that no disruption occurs in any of the emergency commodity consumptions in the time intervals $k T+\tau ;(k+1) T+\tau, l=1, \ldots, r$, with probability $1-\varepsilon$. This parameter, $\varepsilon$, represents the probability of disruption due to the unavailability of the required commodities or disruptions in the transportation system, etc. The optimal values of the second-stage variables $m_{u}^{(l)} \geq 0$ are the adjustment values of the safety stocks. If the safety stock levels are $m_{u}^{(l)} \geq 0$ at time $k T+\tau$, the new stock levels calculated are $m^{(l)}+m_{u}^{(l)} \geq 0, u \in U, l=1, \ldots, r$. Here, the adjustments incur some costs, where the adjustment cost function of an emergency commodity $l$ is denoted by $f^{(l)}(x), l=1, \ldots, r$.

We approximate the joint distribution of the random consumption and delivery variables using an approximate multivariate normal distribution with the random variable $W_{i u}^{(l)}$ for each commodity $l=1, \ldots, r$, for $i=1, \ldots, n$, and for each support $u, u \in U$. Therefore, $W_{i u}^{(l)}$ simply represent the values of the probability distribution of the commodities in terms of consumption minus delivery for any time step:

$$
\begin{gathered}
W_{i u}^{(l)}=i \gamma^{(l)}+Y_{1}^{(l)}+\ldots+Y_{n}^{(l)}-i \delta^{(l)}-X_{1}^{(l)}-\ldots-X_{n}^{(l)}, l=1, \ldots, r, i=1, \ldots, n \\
\text { for each } u, u \in U
\end{gathered}
$$

The expectations, variances, and elements of the covariance matrix for the random variable $W_{i u}^{(l)}$ for each commodity $l=1, \ldots, r$, for $i=1, \ldots, n$, and for each support $u, u \in U$ are calculated based on the normal approximation approach given in Prékopa (2006) as follows:

$$
\mu_{i u}^{(l)}=i \gamma^{(l)}-i \delta^{(l)}+\left(C_{u}^{(l)}-n \gamma^{(l)}\right)\left(\frac{k_{i}^{(l)}}{V^{(l)}+1}\right)-\left(D_{u}^{(l)}-n \delta^{(l)}\right)\left(\frac{h_{i}^{(l)}}{L^{(l)}+1}\right)
$$




$$
\begin{gathered}
\left(\sigma_{i u}^{(l)}\right)^{2}=\left(C_{u}^{(l)}-n \gamma^{(l)}\right)^{2}\left(\frac{k_{i}^{(l)}}{V^{(l)}+1}\right)\left(\frac{1}{V^{(l)}+2}\right)+\left(D_{u}^{(l)}-n \delta^{(l)}\right)^{2}\left(\frac{h_{i}^{(l)}}{L^{(l)}+1}\right)\left(\frac{1}{L^{(l)}+2}\right) \\
\Sigma(\bullet)=\left(D_{u}^{(l)}-n \delta^{(l)}\right)^{2}\left(\frac{-h_{i}^{(l)}\left(h_{f}^{(l)}-h_{i}^{(l)}\right)}{\left(L_{i}^{(l)}+1\right)^{2}\left(L_{i}^{(l)}+2\right)}\right)-\left(C_{u}^{(l)}-n \gamma^{(l)}\right)^{2}\left(\frac{-k_{i}^{(l)}\left(k_{f}^{(l)}-k_{i}^{(l)}\right)}{\left(V_{i}^{(l)}+1\right)^{2}\left(V_{i}^{(l)}+2\right)}\right)
\end{gathered}
$$

where

$l$ : type of commodity, $l=1, \ldots, r$ where $r$ is the number of commodities,

$n:$ number of delivery times in interval $[0, T]$,

$i$ : each delivery time, $i=1, \ldots, n-1$,

$\gamma$ : minimum amount of commodities consumed in a delivery time,

$\delta$ : minimum amount of stock supplied in a delivery time,

$v$ : sample from uniformly distributed population in $\left[0, C_{u}-n \delta\right]$ for each $u, u \in U$

$x$ : sample from uniformly distributed population in $\left[0, D_{u}-n \gamma\right]$ for each $u, u \in U$,

$V$ : sample size of $v$, and $L$ : sample size of $x$,

$k_{i}$ :positive integers selected randomly from the sample $v=1, \ldots, n-1$ to calculate the mean and variance of the consumption distribution,

$k_{f}$ :positive integers selected randomly from the sample $v, 1 \leq i \leq f \leq n-1$, to calculate the covariances of the consumption distribution,

$h_{i}$ :positive integers selected randomly from the sample $v=1, \ldots, n-1$ to calculate the mean and variance of the delivery distribution,

$h_{f}$ :positive integers selected randomly from the sample $v, 1 \leq i \leq f \leq n-1$, to calculate the covariances of the delivery distribution, 
$\Sigma(\bullet)$ : represents any element of the covariance matrix, $\Sigma\left(W_{i u}^{(l)}\right)$.

With this information, an overview of the model that shows the inputs and outputs can be shown as in Table 4.1.

Table 4.1 Inputs and Outputs for the SHIC Model

\begin{tabular}{|c|c|c|c|c|}
\hline $\begin{array}{l}\text { Inputs } \\
n: \text { Number of deliveries } \\
m^{(l)}: \text { Initial safety stock } \\
D_{u}^{(l)}: \text { Total amount of delivery } \\
(\text { amount of commodities) } \\
C_{u}^{(l)}: \text { Total amount of consumption } \\
W_{u}^{(l)}: \text { Approximate normal } \\
\text { distribution variable of the random } \\
\text { consumption and delivery } \\
\text { distributions } \\
g^{(l)}, f^{(l)}, q^{+(l)}, q^{-(l)}: \text { Associated } \\
\text { costs } \\
M: \text { Total capacity } \\
\varepsilon: \text { Probability of disruption }\end{array}$ & $=$ & $\begin{array}{l}\text { SHIC } \\
\text { Model }\end{array}$ & $\Rightarrow$ & $\begin{array}{l}\text { Outputs } \\
m_{u}^{(l)}: \text { Additional amount of } \\
\text { safety stock required to } \\
\text { satisfy the needs for the } \\
\text { vital supplies }\end{array}$ \\
\hline
\end{tabular}

\subsubsection{Constraints}

It is important to consider the probabilistic nature of the consumption and delivery processes, given the high stochasticities of the problem domain dealing with events that will occur just before and after a natural or man-made disaster. Unlike relatively predictable conditions of our daily lives, disaster conditions are highly stochastic. Thus, our model will take these stochasticities into account in terms of probabilistic constraints. 
There are two types of constraints in the model: probabilistic constraints and capacity constraints.

The probabilistic constraints ensure the minimal disruption of the commodities in the shelters with a given probability. Therefore, the sum of initial stocks and deliveries has to be greater than or equal to the consumption for any time step. When we replace $W_{i u}^{(l)}$ 's with their expectations and variances of the approximate normal distribution, our probabilistic constraint is defined as

$$
P\left(W_{i u}^{(l)} \leq m^{(l)}+m_{u}^{(l)}\right) \geq 1-\varepsilon \Rightarrow \prod_{l=1}^{r} \Phi\left(\frac{m^{(l)}+m_{u}^{(l)}-\mu_{i u}^{(l)}}{\sigma_{i u}^{(l)}}, \Sigma\left(W_{i u}^{(l)}\right)\right) \geq 1-\varepsilon
$$

The choice of joint constraints or individual chance constraints is a matter of model formulation and the dynamics of the problem. It may be meaningless to employ a single-commodity analysis when the safety stocks in our problem do affect each other, or when there are multi-suppliers for the same commodity.

Other constraints in the SHIC model are the capacity constraints. At any time step, the initial safety stock plus the optimal additional stock must be smaller than the storage capacity for that commodity, and the sum of storage capacities for each commodity $\left(a^{(l)}\right.$, space occupied by each commodity $l=1, \ldots, r$, multiplied by the storage capacity for each commodity, $M^{(l)}$ ) must be smaller than the total capacity, $M$.

\subsubsection{Objective Function}

The objective cost function is the sum of individual costs listed below: 
- Cost of Storage, $g^{(l)}$ : It is obvious that there is a cost for storing each commodity $l=1, \ldots, r$. In case of disaster operations, it is important to consider storage costs, as the occurrence of a disaster is not known a priori.

- Cost of Surplus, $q^{+(l)}$ : This is incurred if there is more inventory than demand. It can be modeled as a fixed cost or as a step function that allows very low or no cost for a certain surplus level and then a steep increase for higher levels of surplus.

- Cost of Shortage, $q^{-(l)}$ : This cost is incurred if there is not sufficient inventory to satisfy the demand of the evacuees. This is the most important cost component; as clearly seen in the case of Hurricane Katrina, the Haiti earthquake, and other recent disasters, the shortage of vital supplies can even cause loss of life.

- Cost of Adjustment, $f^{(l)}$ : This cost is incurred by the nature of the two-stage model. Suppose we have an initial amount of safety stock, but to satisfy the probability constraint, we need more. This adjustment can be due to unexpected factors such as the strength of a hurricane or an earthquake, an increased number of people who are affected and need help, etc. Of course, this has to be penalized. It can be chosen as a linear function of the additional stock.

The objective function also includes the demands for the consumption of vital commodities multiplied by their corresponding probabilities of occurrence. This allows us to calculate the total cost where the highest and lowest demands have the lowest probabilities according to a pre-determined discretized normal distribution. That is, the total cost of a severe disaster may be higher than others due to the additional safety stocks 
required; however, the probability associated with this high demand will be smaller than lower levels of demand closer to the mean (normal distribution assumption). This selfcontrolling mechanism gives analysts the chance to determine safety stocks more accurately. Intuitively, these disaster probabilities in the objective function represent the uncertainty involved in the demand and supply processes during the disasters. By analyzing different delivery and consumption values with their corresponding probabilities, one can be prepared for different levels of disruptions with different values of disaster probabilities and therefore calculate the optimal initial safety stocks accordingly. This analysis also represents the best and worst case scenarios so that planners and decision makers can be aware of the consequences of overstocking (surplus) and shortage.

\subsubsection{SHIC Formulation}

Using the mathematical information given in the previous subsections, the overall multi-commodity stochastic programming problem is as follows:

$$
\begin{gathered}
\min \left\{\sum_{l=1}^{r} g^{(l)} M^{(l)}+\frac{1}{T} \sum_{u \in U} p_{u}\left[f^{(l)} m_{u}^{(l)}+\sum_{i=1}^{n}\left(q_{i}^{+(l)}+q_{i}^{-(l)}\right) \int_{m^{(l)}+m_{u}^{(l)}}^{\infty}\left(1-\Phi\left(\frac{z-\mu_{i u}^{(l)}}{\sigma_{i u}^{(l)}}\right)\right) d z\right]\right\} \\
\text { Subject to } \\
\prod_{l=1}^{r} \Phi\left(\frac{m^{(l)}+m_{u}^{(l)}-\mu_{i u}^{(l)}}{\sigma_{i u}^{(l)}}, i=1, \ldots, n-1, \Sigma(\bullet)\right) \geq 1-\varepsilon \\
m^{(l)}+m_{u}^{(l)} \leq M^{(l)}, u \in U, l=1, \ldots, r \\
m_{u}^{(l)} \geq 0, u \in U, l=1, \ldots, r \\
\sum_{l=1}^{r} a^{(l)} M^{(l)} \leq M
\end{gathered}
$$

The Hungarian inventory control model, on which our SHIC model is based, was shown to be a convex nonlinear programming problem by Prékopa (2006), assuming that 
the cost functions $g^{(l)}, f^{(l)}, q^{+(l)}, q^{-(l)}$ are convex. During the analysis, $g^{(l)}, q^{+(l)}, q^{-(l)}$ are taken as fixed costs, whereas $f^{(l)}$ is selected as a linear function of the additional safety stock ensuring the convexity. The convexity of the feasible solutions is a consequence of the general theorems on multivariate logconcave measures introduced by Prékopa (1971).

\subsubsection{Proposed Solution Approach}

A solution to the SHIC model requires a complex approach for which the p-level efficient points (pLEPs) methodology developed by Prékopa (1990) is proposed. The pLEPs methodology can be defined as follows:

Definition: Let $\xi$ be a random variable, $Z$ be the set of all possible values of $\xi$, and $F$ be its CDF. A vector $z \in Z$ is a pLEP (p-level efficient point) of the distribution of $\xi$ if $F(z) \geq p, p \in[0,1]$, and there is no $y$ such that $y \in Z, y<z$ and $F(y) \geq p$.

Explanation: $F$ is the cumulative distribution function of the r-dimensional random vector $\xi=\left(\xi_{1}, \ldots, \xi_{r}\right)^{T}$. That is, $F(z) \geq P(\xi \leq z)$. For the r-dimensional vector $z=\left(z_{1}, \ldots, z_{r}\right)^{T}, y<z$ means $y_{q} \leq z_{q}$ for $q=1, \ldots, r$ and $y_{q}<z_{q}$ for at least one $q$.

Theorem: Let $z^{(j)}, j=1, \ldots, N$ be the pLEPs for the probability distribution in $Z=Z_{1} x \ldots x Z_{r}$ and let $F(Z)$ be the CDF of the discrete random vector $\xi$. Then,

$$
P\left(z_{0} \geq \xi\right) \geq p \text { if and only if } P\left(z_{0} \in \bigcup_{j=1}^{N}\left\{z \mid z \geq z^{(j)}\right\}\right)
$$

The pLEPs methodology provides a discretized set of points, which gives the lower bound of a specific probability distribution (Prékopa, 2003). These are used to create the deterministic equivalent of the probabilistic constraints, and they assure that 
the constraints will satisfy the given reliability level, $p=1-\varepsilon$. However, first of all, before applying the pLEPs method, continuous distribution functions in our model have to be converted into approximate discrete distributions (Noyan and Prékopa, 2006). For that purpose, for each demand $u, u \in U$, we approximate the random variable $W_{i u}^{(l)}$ by a discrete variable $\xi_{i u}^{(l)}$ with possible values $\varpi_{u 1}^{(l)}<\varpi_{u 2}^{(l)}<\ldots<\varpi_{u L}^{(l)}$, where the distribution function is:

$$
P\left(W_{i u}^{(l)} \leq m^{(l)}+m_{u}^{(l)}\right) \Rightarrow \Phi\left(W_{i u}^{(l)}\right) \approx F_{W_{i u}^{(l)}}\left(\varpi_{u \vartheta}^{(l)}\right)=\left\{\begin{array}{c}
F_{\xi_{i u}^{(l)}}\left(\varpi_{u \vartheta}^{(l)}\right), \vartheta=1, \ldots, L-1 \\
1, \vartheta=1, \ldots, L-1
\end{array}\right\}, l=1, \ldots, r
$$

Here, $\varpi_{u 1}^{(l)}<\varpi_{u 2}^{(l)}<\ldots<\varpi_{u L}^{(l)}$ are chosen to be equidistant on some interval $\left[0, B_{u}^{(l)}\right]$ where $F_{W_{i l}^{(l)}}\left(B_{u}^{(l)}\right)=1-\zeta$ for a prescribed small tolerance $\zeta$, and $B_{u}^{(l)}$ is the selected upper boundary of the interval of $\varpi_{u i}^{(l)}$ values for each commodity $l=1, \ldots, r$, and for each demand $u, u \in U$. Using this process, we obtain the following probabilistic constraint in the form of multiplication of the cumulative distribution functions of $\xi_{i u}^{(l)}$ :

$$
\prod_{l=1}^{r} F_{\xi_{i u}^{(l)}}\left(\varpi_{u \vartheta}^{(l)}\right) \geq 1-\varepsilon, \vartheta=1, \ldots, L-1, l=1, \ldots, r \text { for each } u, u \in U
$$

That is, we discretize our continuous cumulative distribution function associated with $W_{i u}^{(l)}$ on its entire domain. During the analysis, we try to keep a substantial amount of accuracy while choosing the possible values of $\xi_{i u}^{(l)}$ in the interval $\left[0, B_{u}^{(l)}\right]$ and selecting $B_{u}^{(l)}$ and $N$ accordingly. With this idea, we try to preserve most of the information related to the original function in the discretized one. The important point is that we focus on the upper regions of the distribution function that contribute to the calculation of 
pLEPs with respect to the selected disruption probability. Finally, with the use of this methodology, the relaxation for the multi-commodity problem (SHIC) is performed as follows:

$$
\begin{aligned}
& \text { Original Problem Disjunctive Programming Relaxed Disjunctive } \\
& \min f\left(m_{u}^{(l)}\right) \\
& \text { Problem } \\
& \text { Subject to } \\
& \min f\left(m_{u}^{(l)}\right) \\
& \text { Programming Problem } \\
& \prod_{l=1}^{r} F_{\xi_{i u}^{(l)}}\left(\varpi_{u \vartheta}^{(l)}\right) \geq 1-\varepsilon \\
& \text { Subject to } \\
& \min f\left(m_{u}^{(l)}\right) \\
& m_{u}^{(l)}-M^{(l)} \leq-m^{(l)} \\
& m^{(l)}+m_{u}^{(l)} \geq z^{(j)} \\
& \text { Subject to } \\
& m_{u}^{(l)} \geq 0, u \in U, l=1, \ldots, r \\
& \text { for at least one } j=1, \ldots, N \\
& m^{(l)}+m_{u}^{(l)} \geq \sum_{j=1}^{N} \lambda_{j} z^{(j)} \\
& \vartheta=1, \ldots, L-1 \\
& m_{u}^{(l)}-M^{(l)} \leq-m^{(l)} \\
& \sum_{j=1}^{N} \lambda_{j}=1 \\
& \sum_{l=1}^{r} a^{(l)} M^{(l)} \leq M \\
& \begin{array}{r}
m_{u}^{(l)} \geq 0, u \in U, l=1, \ldots \\
\sum_{l=1}^{r} a^{(l)} M^{(l)} \leq M
\end{array} \\
& m_{u}^{(l)}-M^{(l)} \leq-m^{(l)} \\
& m_{u}^{(l)} \geq 0, u \in U, l=1, \ldots, r \\
& \sum_{l=1}^{r} a^{(l)} M^{(l)} \leq M
\end{aligned}
$$

An illustrative explanation for the relaxation process for $L=4$ can be seen in Figure 4.1. 

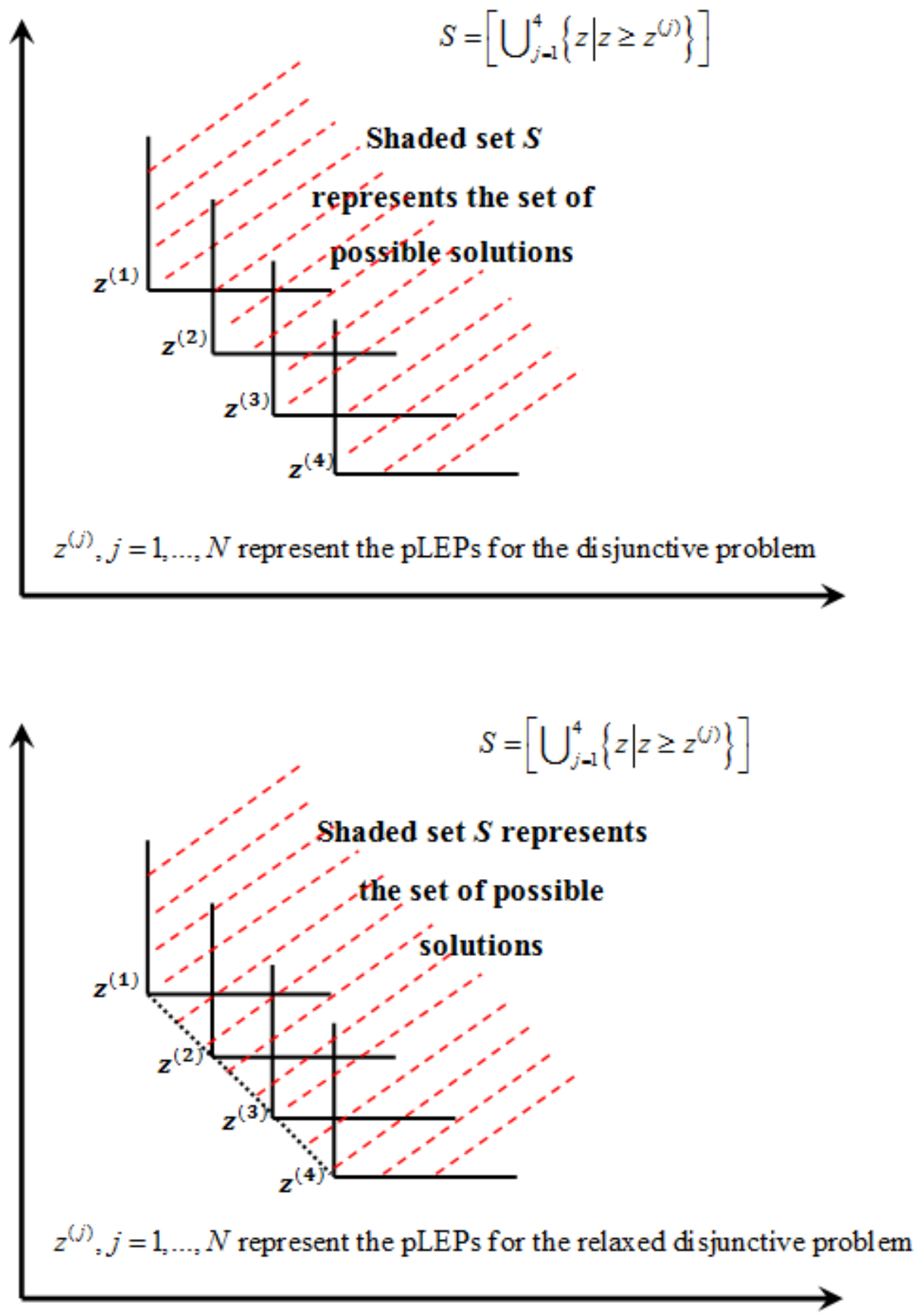

Figure 4.1 pLEPs Method Illustration for Relaxing the Formulation 


\subsubsection{Prékopa-Vizvari-Badics Algorithm (Prékopa, 2010)}

The pLEPs method serves as a practical and easily applicable approximate method so that a decision maker/planner can use the results to decide on the inventory levels for the emergency commodities needed at shelters. To generate the pLEP sets for the relaxed disjunctive programming problem, the Prékopa-Vizvari-Badics algorithm (Prékopa, 2010) will be used. The algorithm is based on the discrete random variable $\xi_{i u}^{(l)}$, where $Z=Z_{1} x \ldots x Z_{r}$ is the product set containing the support of $\xi$, the vector of the discrete random variable $\xi_{i u}^{(l)}$. For the sake of illustration, we consider the rdimensional vector as $\xi=\left(\xi_{1}, \ldots, \xi_{r}\right)^{T}$. The algorithm is as follows:

Step 0. Initialize $k \leftarrow 0$.

Step 1. Determine $z_{1, j_{1}}, \ldots, z_{r, j_{r}}$ such that

$$
\begin{gathered}
z_{1, j_{1}}=\arg \min \left\{y \mid F\left(y, z_{2, k_{2}+1}, \ldots, z_{r, k_{r}+1}\right) \geq 1-\varepsilon\right\} \\
z_{2, j_{2}}=\arg \min \left\{y \mid F\left(z_{1, j_{1}}, y, z_{3, k_{3}+1}, \ldots, z_{r, k_{r}+1}\right) \geq 1-\varepsilon\right\} \\
\vdots \\
z_{r, j_{r}}=\arg \min \left\{y \mid F\left(z_{1, j_{1}}, \ldots, z_{r-1, k_{r-1}+1}, y\right) \geq 1-\varepsilon\right\}
\end{gathered}
$$

and let $E \leftarrow\left\{z_{1, j_{1}}, \ldots, z_{r, j_{r}}\right\}$.

Step 2. Let $k \leftarrow k+1$. If $j_{1}+k>k_{1}+1$, then go to Step 4. Otherwise, go to Step 3 .

Step 3. Enumerate the pLEPs of the function $F\left(z_{1, j_{j}+k}, y\right), y \in R^{r-1}$ based on the discretization within the interval $\left[0, B_{u}^{(l)}\right]$ as described in the previous section, and eliminate those that dominate at least one element in $E(y$ dominates $z$ if 
$y \geq z, y \notin z$ ). If $H$ is the set of the remaining pLEPs, then let $E \leftarrow E \cup H$. Go to

Step 2.

Step 4. Stop; $E$ is the set of all pLEPs of the CDF $F(z)=P(\xi \leq z)$.

In the proceeding section, we will present the results obtained by solving the SHIC problem starting with a single-commodity case.

\subsection{Single-Commodity Results}

In this section, we will present the single-commodity case of the problem. This case enables us to study the impact of model parameters such as the probabilistic consumption and delivery factors, initial stock levels, etc. without loss of generality on the single-commodity SHIC model.

\subsubsection{Single-Commodity SHIC Formulation}

The single commodity mathematical formulation is as follows:

$$
\begin{gathered}
\min \left\{g(M)+\frac{1}{T} \sum_{u \in U} p_{u}\left[f\left(m_{u}\right)+\sum_{i=1}^{n} q_{i}^{-}+\sum_{i=1}^{n}\left(q_{i}^{+}+q_{i}^{-}\right) \int_{m+m_{u}}^{\infty}\left(1-\Phi\left(\frac{z-\mu_{i u}}{\sigma_{i u}}\right)\right) d z\right]\right\} \\
\Phi\left(\frac{m+m_{u}-\mu_{i u}}{\sigma_{i u}}, i=1, \ldots, n-1, \Sigma(\bullet)\right) \geq 1-\varepsilon \\
m+m_{u} \leq M, m_{u} \geq 0, u \in U
\end{gathered}
$$

Accurate statistical distributions of delivery and consumption processes are needed to solve and study the above model. In this paper, due to lack of such realistic data, pre-determined discretized normal distributions are adopted. The problem can also be solved for a range of demand values, allowing us to observe changes in safety stock 
values for different consumption amounts. In a post-disaster condition, the requirements of people can drastically change. Therefore, sometimes, it is logical to choose an initial safety stock that may increase the inventory cost but can prevent disruption due to stochastic demand changes. Analysis with different demands of evacuees will enable us to assess the overall picture more clearly.

\subsubsection{Single-Commodity SHIC Base Case Scenario}

A base condition is established with the following parameter values chosen:

- $\varepsilon$ is selected as 0.1 so that the probability $1-\varepsilon$ in the constraint is equal to 0.9 .

- The number of deliveries in the time interval (i.e., a day) is chosen as $n=10$, and the parameters $\delta$ and $\gamma$ are taken to be 0.2 and 0.25 units, respectively.

- The amount of initial safety stock, $m$, is 2 units.

- The surplus and the shortage costs are chosen $q^{+}=0.1 /$ unit and $q^{-}=0.1 /$ unit

- The cost of adjustment function is selected as $f(x)=2 x$.

- The cost of storage for each commodity is $1 /$ unit .

- Total storage capacity is considered to be 20 units.

- The space occupied by each item is 2 units.

- The delivery (demand) and consumption values are taken as $D=[3.2,3.4,3.6,3.8,4.0,4.2]$ and $D=[5.2,5.4,5.6,5.8,6.0,6.2]$, respectively. The probability values of the discrete supports of consumption and delivery 
values are taken as $p=[0.13,0.17,0.20,0.20,0.17,0.13]$ where a numerical study with smaller discrete sections might be useful from a computational analysis point of view, especially for the sensitivity analyses that will be conducted in this section.

The results obtained for the base case are given in Table 4.2. According to Table 4.2, to satisfy the needs of the impacted people $90 \%$ of the time, initial stock has to be more than $50 \%$ of the total expected consumption. That is, having more than half of the commodities in the inventory before the disaster, we will prevent disruption $90 \%$ of the time. Of course, this result is only for the base case. By changing the parameters, it is possible to have higher additional safety stock values and costs. For example, it may not be possible to make the required amount of deliveries in a given period of time, so the additional amount of safety stock will increase, incurring higher costs.

Table 4.2 Results for the Base Case $(m=2)$

\begin{tabular}{|c|c|c|c|c|c|c|}
\hline Demand & $\mathbf{u}=\mathbf{1}$ & $\mathbf{u}=\mathbf{2}$ & $\mathbf{u}=\mathbf{3}$ & $\mathbf{u}=\mathbf{4}$ & $\mathbf{u}=\mathbf{5}$ & $\mathbf{u = 6}$ \\
\hline Initial Stock & 2.00 & 2.00 & 2.00 & 2.00 & 2.00 & 2.00 \\
\hline Optimal Additional Stock & 0.86 & 0.95 & 1.04 & 1.13 & 1.21 & 1.31 \\
\hline Total Initial Stock & 2.86 & 2.95 & 3.04 & 3.13 & 3.21 & 3.31 \\
\hline $\begin{array}{c}\text { Safety Stock to Total Expected } \\
\text { Consumption }\end{array}$ & $55 \%$ & $55 \%$ & $54 \%$ & $54 \%$ & $54 \%$ & $53 \%$ \\
\hline $\begin{array}{c}\text { Total Expected } \\
\text { Consumption }\end{array}$ & 5.20 & 5.40 & 5.60 & 5.80 & 6.00 & 6.20 \\
\hline $\begin{array}{c}\text { The Optimal Value } \\
\text { Thopon of Initial }\end{array}$ & & \multicolumn{3}{|c|}{6.99} & & \\
\hline
\end{tabular}

In the following sensitivity analysis results, values of every parameter are kept the same as in the base case, except as mentioned otherwise. The additional safety stock value changes are given for three demand values. The demand values are in ascending 
order, where demand type 1 represents the lowest demand and demand type 6 represents the highest demand.

\subsubsection{Single-Commodity SHIC Sensitivity Analysis Results}

A sensitivity analysis is conducted by changing the model parameters to observe the behavior of the proposed model.

\subsubsection{Changes in the Probability of Disruption}

To see the behavior of the proposed model, epsilon value, $\varepsilon$, is changed from 0.05 to 0.45 so that the probability of not serving the evacuees is increased. In other words, the probability of satisfying the requirements of all the evacuees is decreased from $95 \%$ to $55 \%$ of the time. The impact of all the changes is shown in Figure 4.2, where the additional safety stock decreases as the probability bound is relaxed. In this case, the total cost reaches a value including only the cost of initial stock, as there is no additional safety stock required in the end. That is, the cost incurred in the end is only due to the storage cost of initial safety stock. As the cost of storage for one stock is chosen as 1 , the resulting cost is the same as the initial safety stock amount. However, it is possible to increase the importance of the initial safety stock by changing the unit cost of storage. Occupied spaces for safety stocks also decrease, as they have a positive correlation with the safety stocks.

Another important issue is studying the model with respect to different distributions. By changing the mean and variance of the normal distribution used in the base model, we can have a better picture of the impact of the probabilistic occurrences. 
These changes can be thought of as a way of representing the severity of the disaster. For a Category 5 hurricane, the requirements of the evacuees can be very high, or the daily demand for medicine can have very high variances depending upon the time-dependent impact of the disaster. To get insight into the above situation, two different distributions are analyzed (Figure 4.2). As observed, a severe hurricane having twice the demand of the base case requires more safety stock, leading to higher costs.

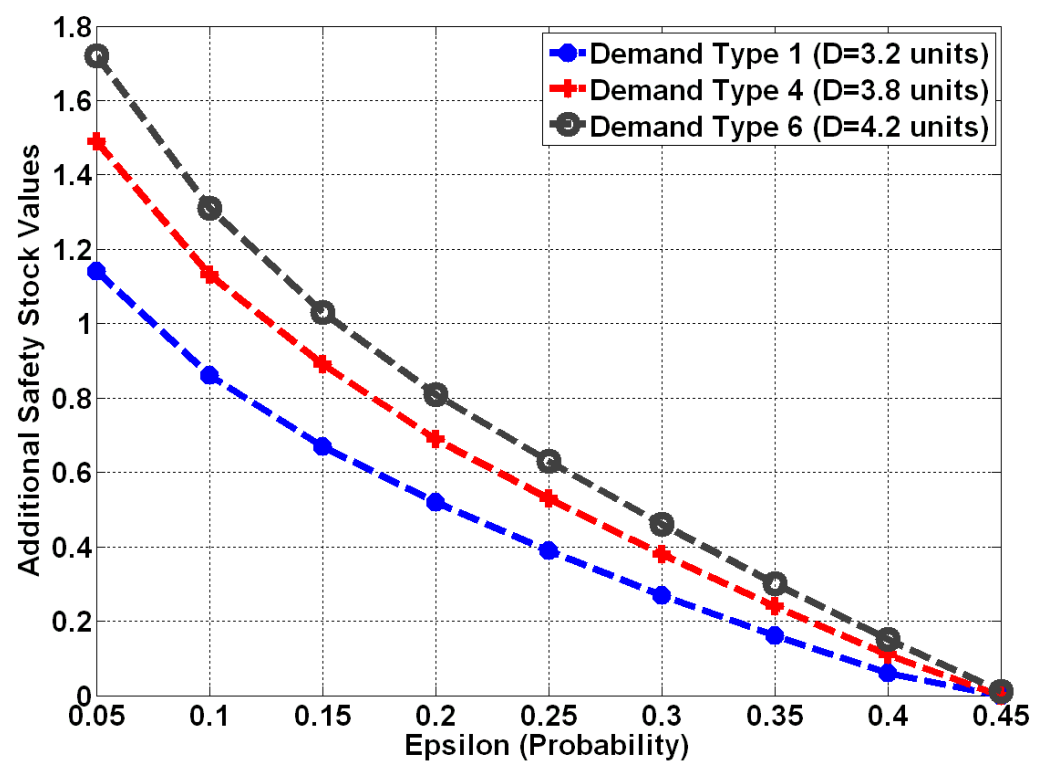

(a) 


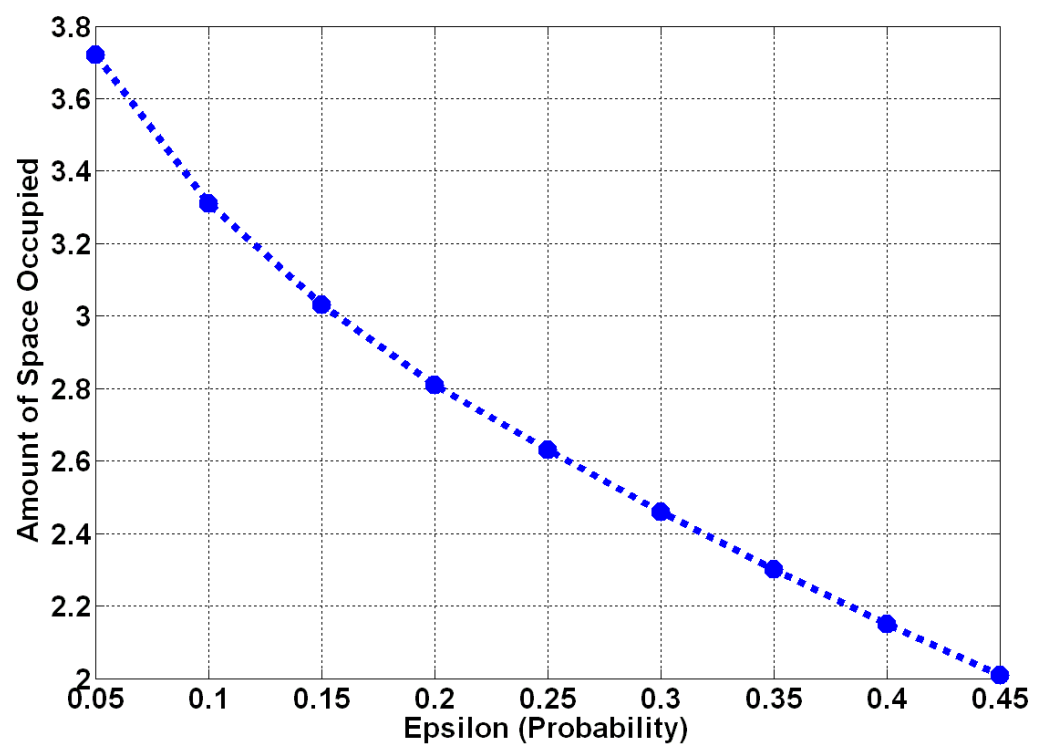

(b)

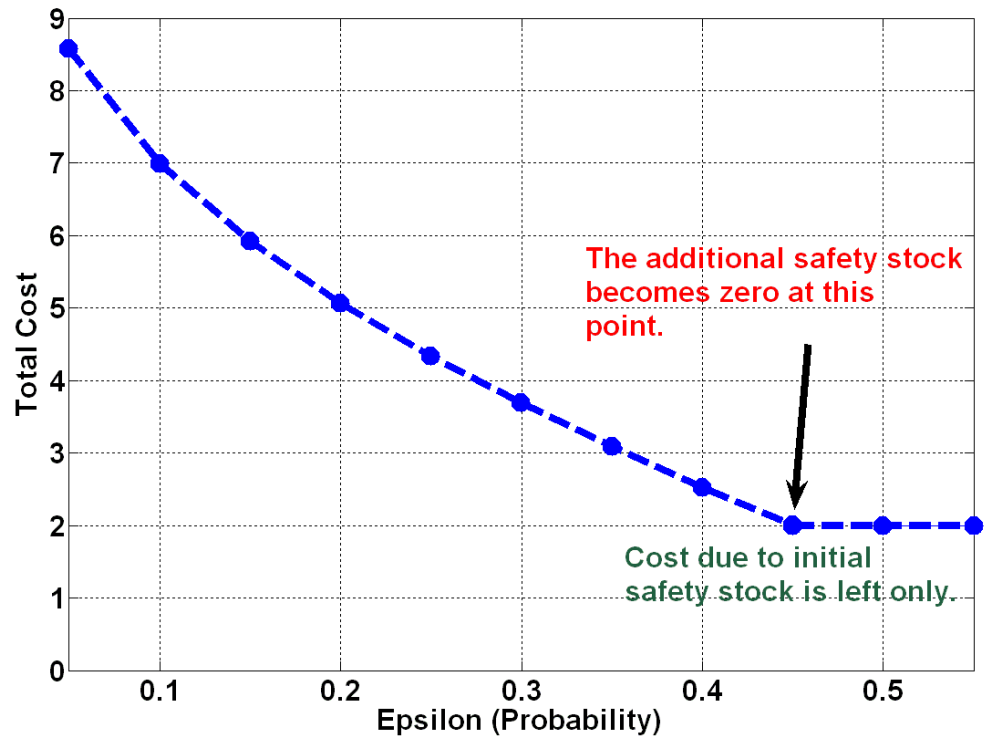

(c) 


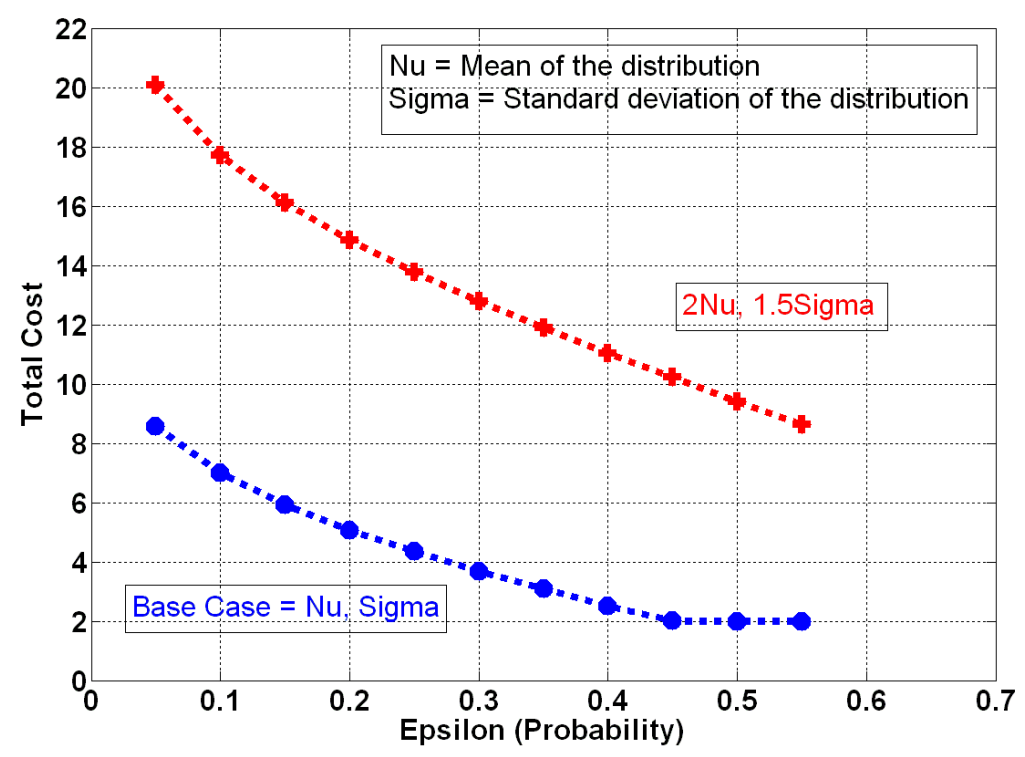

(d)

Figure 4.2 Changes in Additional Safety Stock Values (Figure 4.2.a), Occupied Spaces of Safety Stocks (Figure 4.2.b), Total Cost for the Base Case (Figure 4.2.c) and Total Costs for Different Distributions (Figure 4.2.d) for Different Probabilities

\subsubsection{Changes in the Initial Safety Stock}

The main purpose of the model is to find the safety stock value that minimizes the cost while preventing disruption for a pre-determined probability distribution. Therefore, it is important to observe the behavior of additional stocks denoted as $m_{u}$, as the initial safety stock, $m$, is increased. As expected, $m_{u}$ decreases as $m$ increases. Then, the total cost tends to reach the one including only the storage cost of initial safety stock, as no costs come from the additional safety stock values obtained to satisfy the probabilistic constraint given in Equation (4.9). The behavior of our model in response to an increase in the initial safety stock values can be seen in Figure 4.3. Using these results, the decision maker has to determine the initial safety stock considering the total cost and the possibility of higher demand levels. For devastating localized and sudden onset disasters 
such as the recent earthquakes in Haiti or Japan, the initial safety stocks can be selected as higher than the optimal values, to be on the safe side.

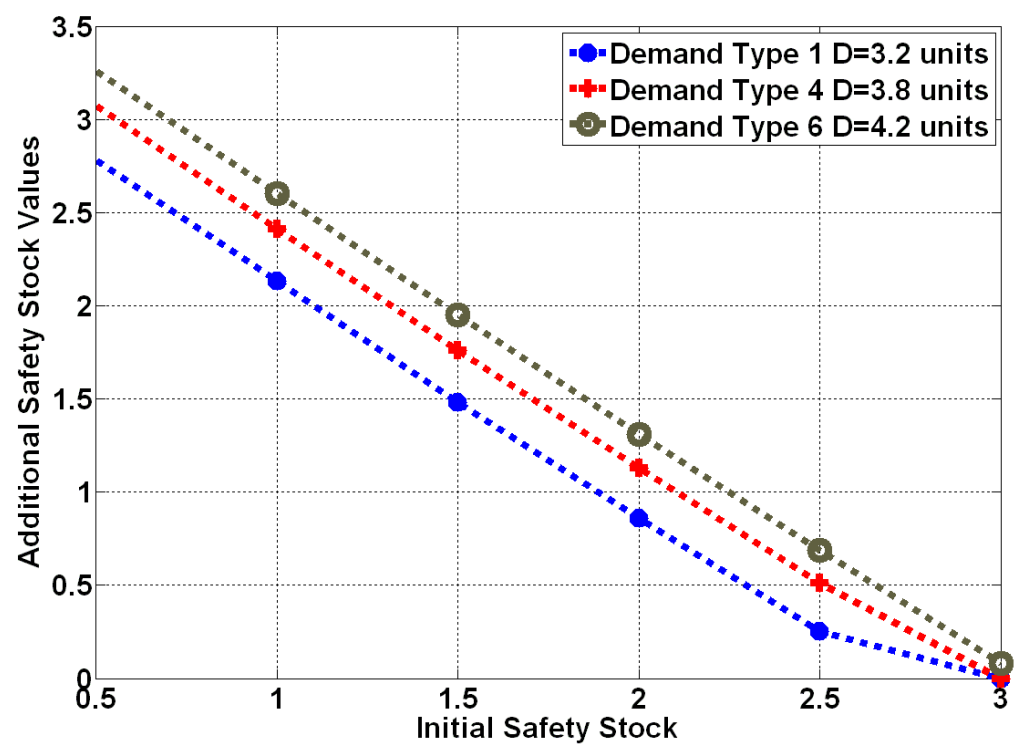

(a)

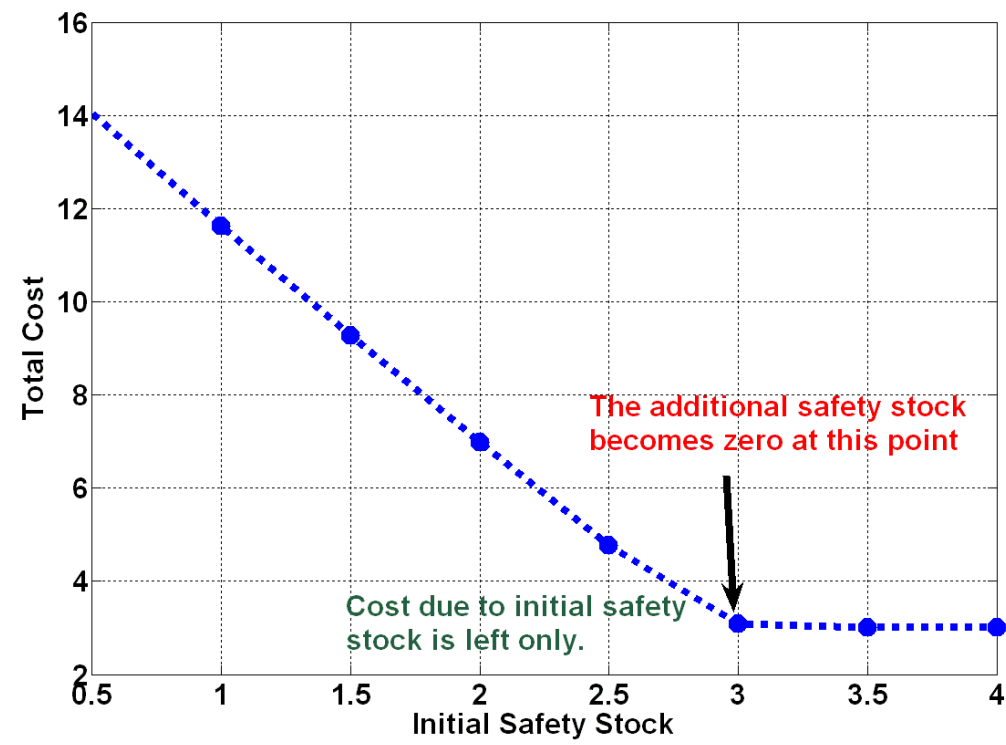

(b)

Figure 4.3 Changes in Additional Safety Stock Values (Figure 4.3.a) and Total Cost (Figure 4.3.b) versus Initial Stocks 


\subsubsection{Changes in the Number of Deliveries}

The number of deliveries during the chosen interval, such as a day or month, has the utmost importance for our model. In the case of disasters, changes in the number of deliveries mainly due to the characteristics of the disaster, increased demand, reduced supply, and possible disruptions in the transportation system are expected. Hence, it is significant to see how the model behaves due to changes in the number of deliveries. The results can be seen in Figure 4.4. As the amount of deliveries is increased, the additional amount of safety stock values tends to decrease. This is a logical behavior, as the increase in the initial safety stock leads to lower additional stock values to satisfy the probability constraint. For instance, when the number of deliveries reaches 22 for the whole time interval in this case, there is no need for additional safety stock. This shows that the initial safety stock is large enough to satisfy the demand of evacuees. Hence, total cost incurred decreases with lower levels of additional safety stocks. If the system is highly stochastic, then the probabilistic constraints are satisfied only if additional safety stock is high, too. Of course, this will impose higher costs. On the other hand, if the initial safety stock is too low, then the problem will become infeasible. Thus, our model achieves a balance between satisfying probability constraints and keeping the cost low.

For instance, especially for dispersed and/or sudden onset disasters that spread over a large area, the realization of the increase in the number of deliveries may not be possible initially due to lack of trucks, personnel, and the commodity itself, or due to transportation-related problems such as possible loss of vital connectors or links. However, the number of deliveries for slow onset disasters such as famine and drought may be more critical, as these rare disasters cause more losses and therefore affect the 
lives of people more than others. Here, again, the critical point is to be able to prepare for the worst-case scenarios where deliveries may not occur as they should, and therefore to have an initial safety stock that will prevent disruption of vital supplies for victims. Therefore, by using the results of this analysis, it is possible to choose the safety stock value corresponding to the number of deliveries that can be comfortably achieved in the aftermath of a major extreme event such as a hurricane or an earthquake.

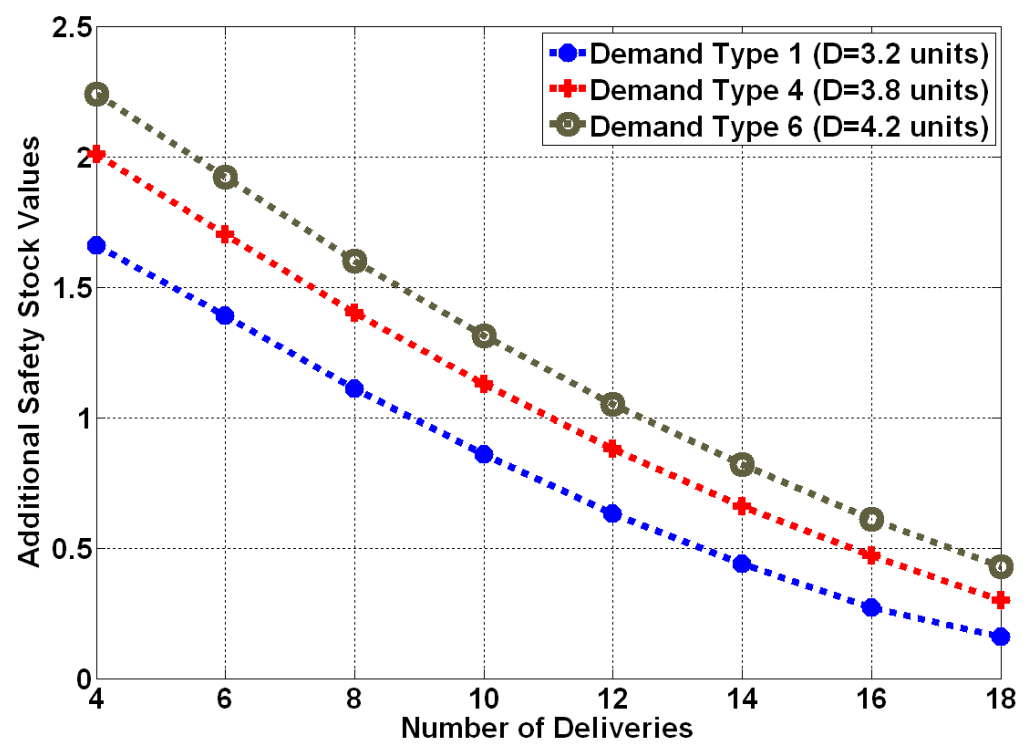

(a) 


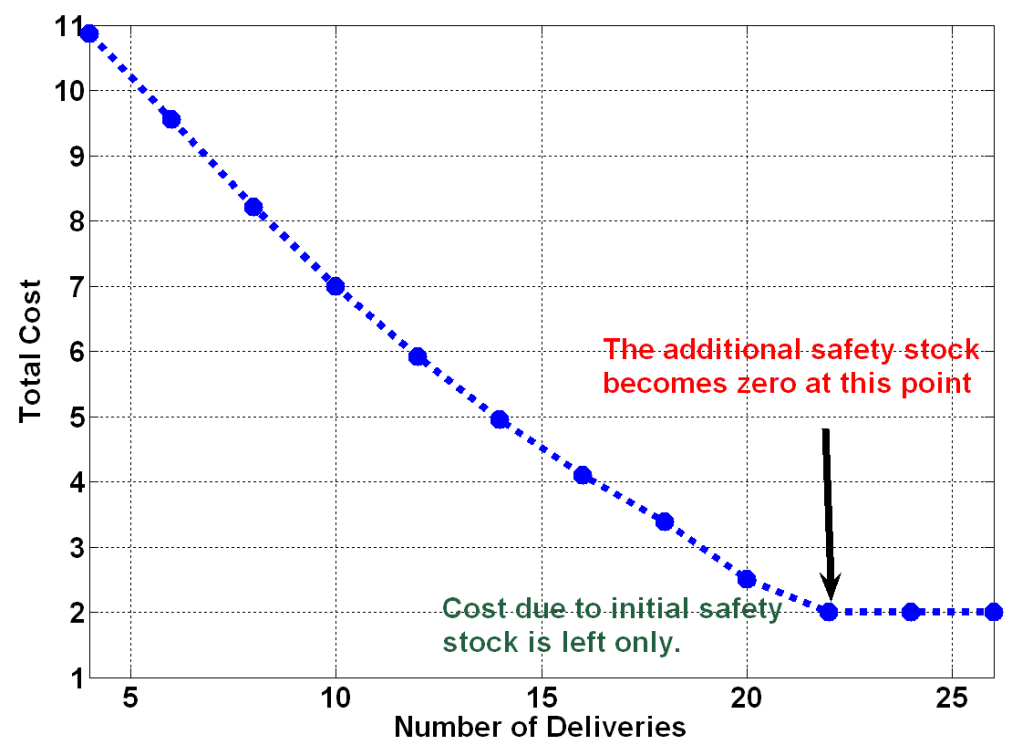

(b)

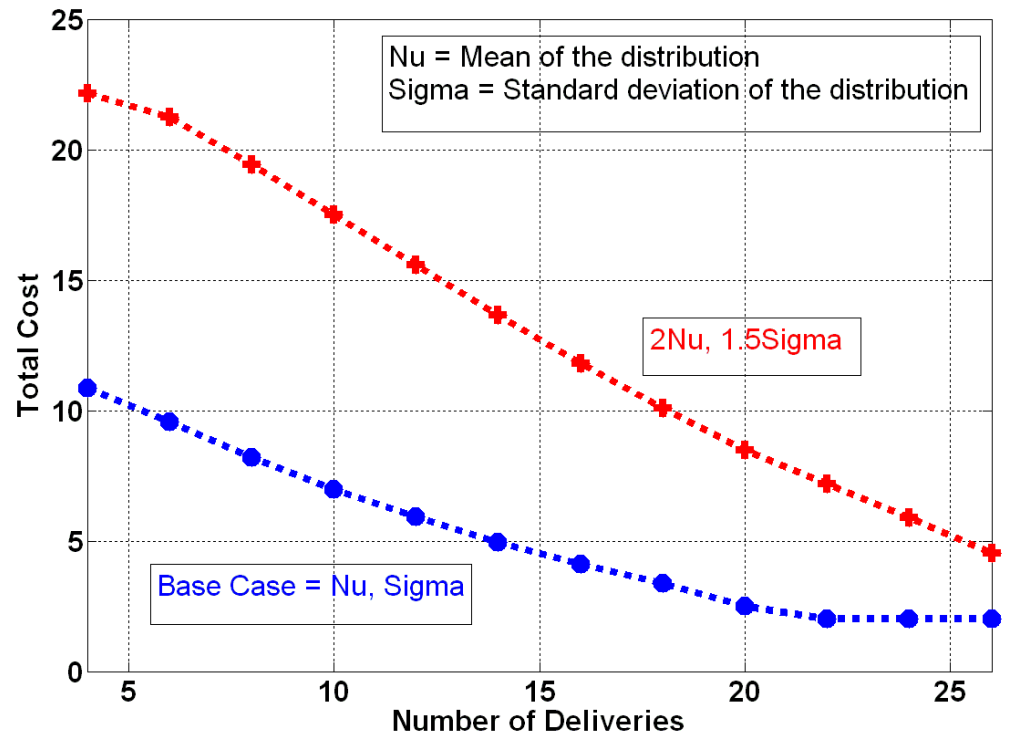

(c)

Figure 4.4 Changes in Additional Safety Stock Values (Figure 4.4.a), Total Cost for the Base Case (Figure 4.4.b) and Total Cost for Two Different Distributions (Figure 4.4.c) versus Changes in the Number of Deliveries 
The behavior of the system is also observed for two different distributions as shown in Figure 4.4. The main question is the following: "How does total cost change according to different demands and number of deliveries?" The answer is that there is a significant amount of difference in the safety stock values as the severity of the disaster increases, where more safety stock results in a substantial amount of increase in the total cost.

\subsubsection{Changes in the Amount of Consumption}

For any disaster type, demand of the victims can vary with time. By changing these consumption values depending on the severity of the disaster, it is possible to figure out the changes in the initial safety stock values. In other words, the mean and variance of our random variable $W_{i u}^{(l)}$ is increased to be able to model the impact of the severity of the disaster as far as the demand and consumption values are concerned. Therefore, the consumption values are increased to $C=[7.2,7.4,7.6,7.8,8.0,8.2]$, and the amount of consumption in one period, $\gamma$, is taken as 0.45 . The results are shown in Table 4.3. When the expected consumption is increased, the initial stock level chosen as 2 units appears to be quite low. Therefore, the additional safety stock levels are higher than expected, leading to higher percentages of initial stock compared with the consumption values. The analysis is conducted one more time, increasing $m$ by 2 units. Apparently, it is logical to have a higher initial safety stock for this case, as the cost associated becomes lower. 
Table 4.3 Results for the Increased Consumption Case

\begin{tabular}{|c|c|c|c|c|c|c|}
\hline \multicolumn{7}{|c|}{ Results for the increased consumption case $(m=2)$} \\
\hline Demand & $\mathbf{u}=\mathbf{1}$ & $\mathbf{u}=\mathbf{2}$ & $\mathbf{u}=\mathbf{3}$ & $\mathbf{u}=\mathbf{4}$ & $\mathbf{u}=\mathbf{5}$ & $\mathrm{u}=\mathbf{6}$ \\
\hline Initial Stock & 2.00 & 2.00 & 2.00 & 2.00 & 2.00 & 2.00 \\
\hline Optimal Additional Stock & 2.86 & 2.95 & 3.04 & 3.13 & 3.22 & 3.31 \\
\hline Total Initial Stock & 4.86 & 4.95 & 5.04 & 5.13 & 5.22 & 5.31 \\
\hline $\begin{array}{l}\text { Proportion of Initial Safety Stock } \\
\text { to Total Expected Consumption }\end{array}$ & $68 \%$ & $67 \%$ & $66 \%$ & $66 \%$ & $65 \%$ & $65 \%$ \\
\hline Total Expected Consumption & 7.20 & 7.40 & 7.60 & 7.80 & 8.00 & 8.20 \\
\hline The Optimal Value & \multicolumn{6}{|c|}{15.79} \\
\hline \multicolumn{7}{|c|}{ Results for the increased consumption case $(m=4)$} \\
\hline Demand & $\mathbf{u}=\mathbf{1}$ & $\mathbf{u}=\mathbf{2}$ & $\mathbf{u}=\mathbf{3}$ & $\mathrm{u}=4$ & $\mathbf{u}=\mathbf{5}$ & $\mathrm{u}=6$ \\
\hline Initial Stock & 4.00 & 4.00 & 4.00 & 4.00 & 4.00 & 4.00 \\
\hline Optimal Additional Stock & 0.86 & 0.95 & 1.04 & 1.13 & 1.22 & 1.31 \\
\hline Total Initial Stock & 4.86 & 4.95 & 5.04 & 5.13 & 5.22 & 5.31 \\
\hline $\begin{array}{l}\text { Proportion of Initial Safety Stock } \\
\text { to Total Expected Consumption }\end{array}$ & $68 \%$ & $67 \%$ & $66 \%$ & $66 \%$ & $65 \%$ & $65 \%$ \\
\hline Total Expected Consumption & 7.20 & 7.40 & 7.60 & 7.80 & 8.00 & 8.20 \\
\hline The Optimal Value & \multicolumn{6}{|c|}{8.99} \\
\hline
\end{tabular}

Figure 4.5 shows the changes in additional safety stock and cost due to the change in average consumption amount, starting from the case where delivery equals consumption. As consumption amounts for the evacuees increase, the safety stock and the corresponding cost also increase. This analysis may be more helpful for slow onset disasters where the severity of the disaster gradually increases, such as Hurricane Katrina. As the disaster intensifies, the number of people located in the shelters will increase, and therefore the needs for vital supplies will also be higher than before. Therefore, this analysis can be used as a means of planning and preparedness for these type of disasters by planners and decision makers. 


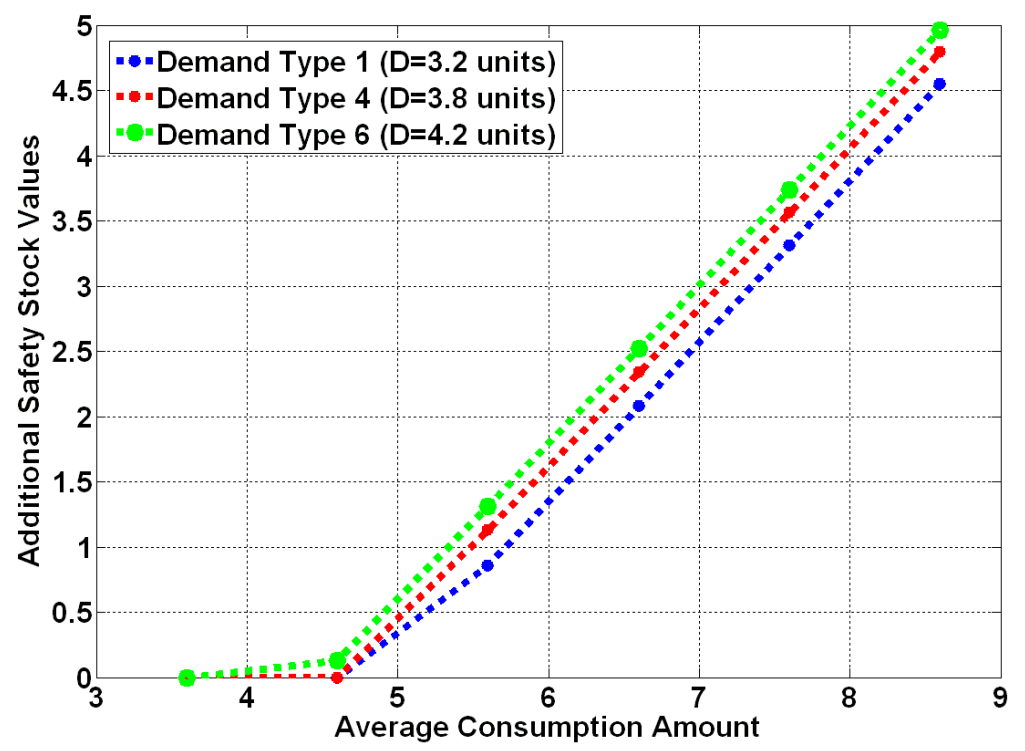

(a)

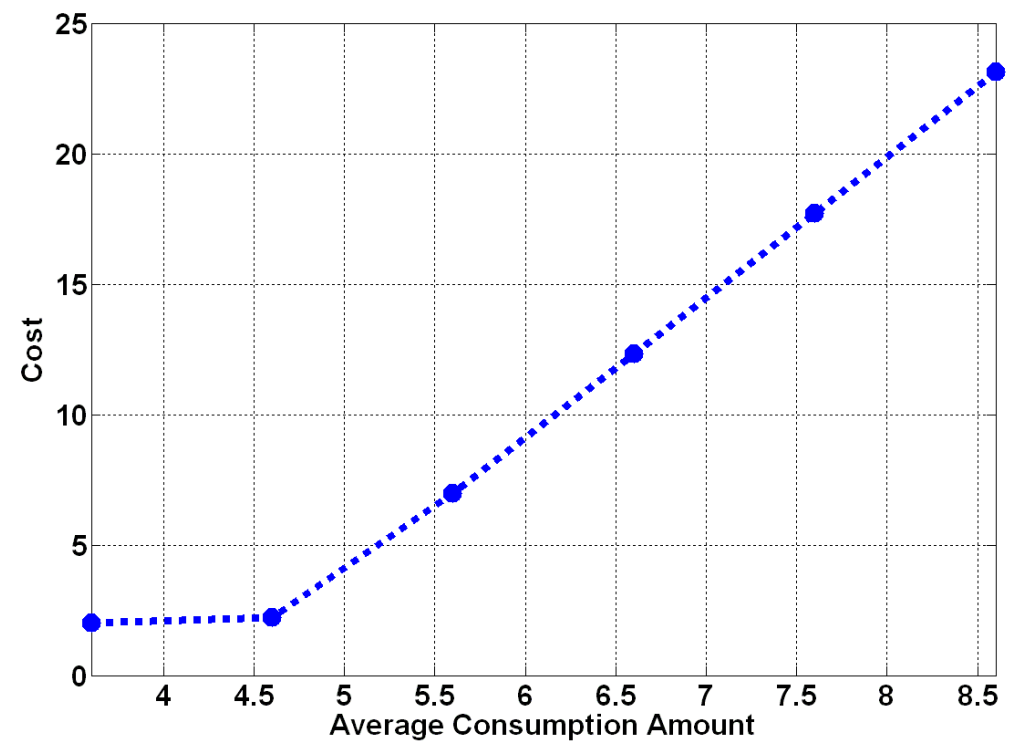

(b)

Figure 4.5 Changes in Additional Safety Stock Values (Figure 4.5.a) and Total Cost (Figure 4.5.b) versus Changes in Average Consumption Amounts 


\subsection{Multi-Commodity Case Studies}

As is obvious from the literature review chapter of this dissertation, there are a large number of studies dealing with different aspects of emergency management and relief operations. The main focus of this section is to demonstrate the importance of the proposed secure and efficient humanitarian inventory management system through the use of realistic case studies built using information available from past disasters. It is clear that a humanitarian inventory management system should be robust with respect to disruptions to minimize the impact of the severity of a particular disaster on the supply and consumption of vital commodities. Our ultimate goal is to use realistic case studies to emphasize this simple yet very important requirement. Based on this approach, we will focus on a case based two-commodity application (Ozguven and Ozbay, 2011) in the following subsections, starting with an example numerical study for the model.

\subsubsection{Example Numerical Study for the MC-SHIC Model}

First, similar to the single-commodity approach, a two-commodity numerical example is created where independent distributions of medicine and MRE's (mealsready-to-eat) are considered. Depending on the severity of the disaster, there are ten demand scenarios given in ascending order, where demand type $1(u=1)$ represents the lowest demand (best case), and demand type $10(\mathrm{u}=10)$ is the highest demand (worst case). The demand for MRE's starts lower than the demand for medicine but increases more rapidly as the severity of the disaster increases. For the base case scenario, the following values are chosen: 
- $\varepsilon$ is $0.05, \zeta$ is $0.01, N$ is 100 , and $B_{u}^{(l)}$ is 100 for all cases.

- Number of deliveries in the time interval (i.e., a day) is $n=4$.

- Amounts of initial safety stock, $m^{(1)}$ and $m^{(2)}$, are 20 and 20 units, respectively.

- $q^{+(1)}=1.2 /$ unit, $q^{+(2)}=1.0 /$ unit , and $q^{-(1)}=120 /$ unit, $q^{-(2)}=100 /$ unit .

- $\quad \delta^{(1)}, \delta^{(2)}, \gamma^{(1)}$ and $\gamma^{(2)}$ are calculated for each commodity separately.

- Cost of adjustment function is selected as $f(x)=2 x$.

- Costs of storage for each commodity are 1.2/unit and 1/unit, respectively.

- Total storage capacity is 100 units.

- $\quad$ Spaces occupied by commodities are 1/unit and 1.2/unit, respectively.

- Expected total delivery and expected total consumption values are taken as

$$
\begin{array}{cc}
D^{1}=[10,12,14,16,18,20,22,24,26,28] & C^{1}=[30,32,34,36,38,40,42,44,46,48] \\
D^{2}=[0,4,8,12,16,20,24,28,32,36] & C^{2}=[20,24,28,32,36,40,44,48,52,56]
\end{array} .
$$

- Probabilities of the discrete supports of consumption and delivery values are

$$
p=[0.03,0.06,0.10,0.14,0.17,0.17,0.14,0.06,0.03]
$$

The results are given in Table 4.4. According to these results, to satisfy the needs of the victims $95 \%$ of the time, initial safety stock must be at least more than $71 \%$ of total expected consumption for medical supplies. That is, having more than $71 \%$ of the commodities in the inventory before the disaster will prevent disruption $95 \%$ of the time. For the MRE's, the initial stock must be more than $75 \%$ of total expected consumption. This indicates the importance of correctly determining the initial safety stocks in the emergency inventory, as having this percentage of stocks will prevent disruption $95 \%$ of the time. When the total expected consumption is equal to the initial safety stock, the 
model finds the optimal additional safety stock value as zero. Moreover, for this example, it may not be possible to make 4 deliveries in a given period of time; thus, the additional amount of safety stock has to increase.

Table 4.4 Results for the Two-Commodity Numerical Example

\begin{tabular}{|c|c|c|c|c|c|c|c|c|c|c|}
\hline Demand & $\mathbf{u}=\mathbf{1}$ & $\mathbf{u}=\mathbf{2}$ & $\mathbf{u}=\mathbf{3}$ & $\mathbf{u}=\mathbf{4}$ & $\mathbf{u}=\mathbf{5}$ & $\mathbf{u = 6}$ & $\mathbf{u}=\mathbf{7}$ & $\mathbf{u}=\mathbf{8}$ & $\mathbf{u = 9}$ & $\mathbf{u = 1 0}$ \\
\hline $\begin{array}{c}\text { Initial Stock } \\
\text { (Medicine) }\end{array}$ & 20 & 20 & 20 & 20 & 20 & 20 & 20 & 20 & 20 & 20 \\
\hline $\begin{array}{c}\text { Optimal Additional } \\
\text { Stock } \\
\text { (Medicine) }\end{array}$ & 2 & 3 & 5 & 6 & 8 & 10 & 11 & 12 & 13 & 15 \\
\hline $\begin{array}{c}\text { Total Initial Stock } \\
\text { (Medicine) }\end{array}$ & 22 & 23 & 25 & 26 & 28 & 30 & 31 & 32 & 33 & 35 \\
\hline $\begin{array}{c}\text { Total Expected } \\
\text { Consumption } \\
\text { (Medicine) }\end{array}$ & 30 & 32 & 34 & 36 & 38 & 40 & 42 & 44 & 46 & 48 \\
\hline $\begin{array}{c}\text { Proportion of Initial } \\
\text { Safety Stock to Total } \\
\text { Expected } \\
\begin{array}{c}\text { Consumption } \\
\text { (Medicine) }\end{array}\end{array}$ & 73.3 & 71.9 & 73.5 & 72.2 & 73.7 & 75.0 & 73.8 & 72.7 & 71.7 & 72.9 \\
\hline Initial Stock (MRE) & 20 & 20 & 20 & 20 & 20 & 20 & 20 & 20 & 20 & 20 \\
\hline $\begin{array}{c}\text { Optimal Additional } \\
\text { Stock (MRE) }\end{array}$ & 0 & 1 & 2 & 4 & 7 & 11 & 13 & 16 & 20 & 22 \\
\hline $\begin{array}{c}\text { Total Initial Stock } \\
\text { (MRE) }\end{array}$ & 20 & 21 & 22 & 24 & 27 & 31 & 33 & 36 & 40 & 42 \\
\hline $\begin{array}{c}\text { Total Expected } \\
\text { Consumption } \\
\text { (MRE) }\end{array}$ & 20 & 24 & 28 & 32 & 36 & 40 & 44 & 48 & 52 & 56 \\
\hline $\begin{array}{c}\text { Proportion of Initial } \\
\text { Safety Stock to Total } \\
\text { Expected }\end{array}$ & 100.0 & 87.5 & 78.6 & 75.0 & 75.0 & 77.5 & 75.0 & 75.0 & 76.9 & 75.0 \\
\hline $\begin{array}{c}\text { Consumption } \\
\text { (MRE) }\end{array}$ & $\%$ & $\%$ & $\%$ & $\%$ & $\%$ & $\%$ & $\%$ \\
\hline
\end{tabular}

Using this methodology, we will first introduce the proposed case-based inventory management model. This case study-based approach requires certain steps, including the creation of demand and supply distributions and parameter selection, which are methodologically introduced in Figure 4.6. Using these steps, planners and decision makers can create case studies based on real-life experience from previous disasters. 


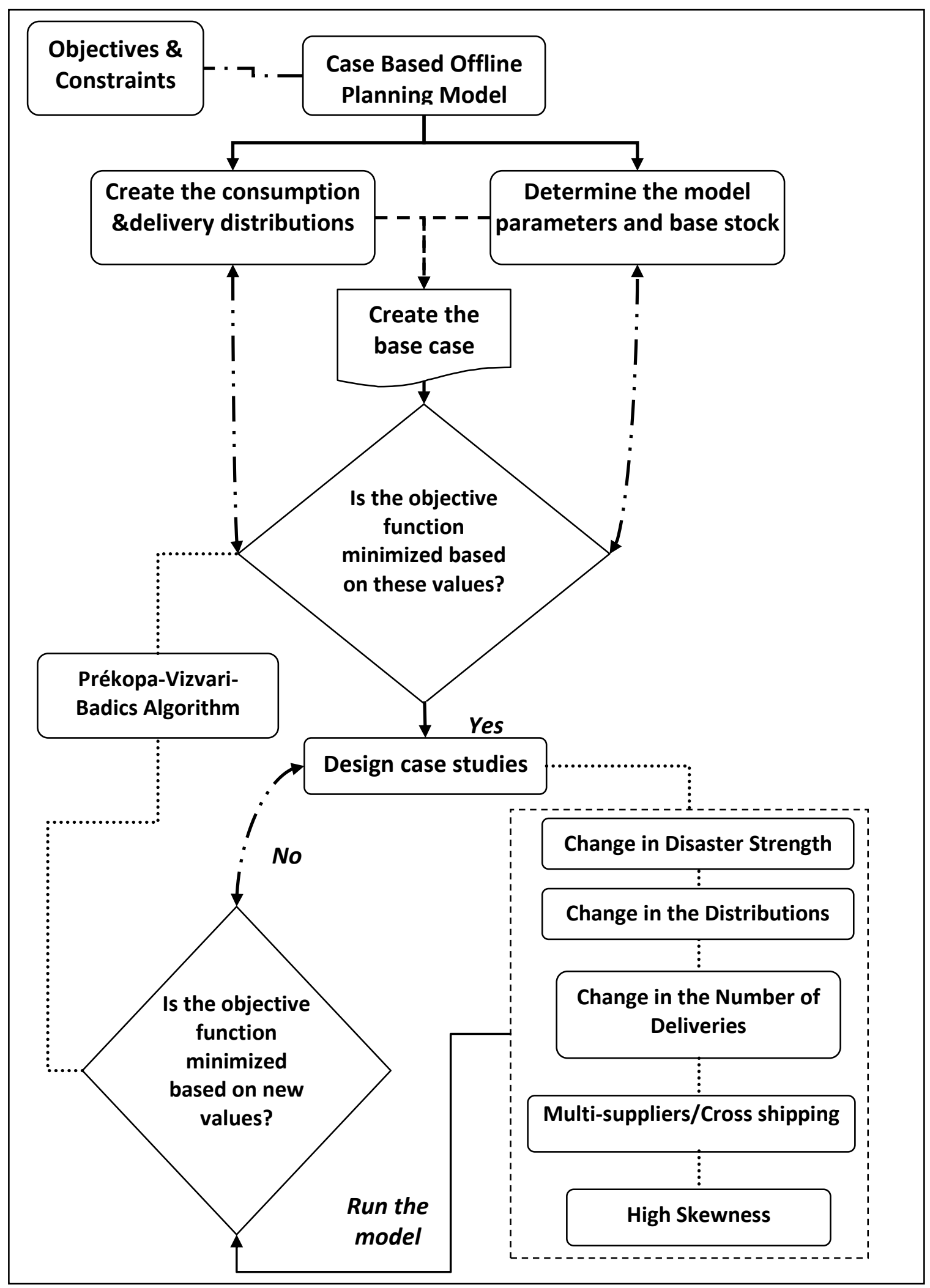

Figure 4.6 Overall Methodology for the Case Based Inventory Management 


\subsubsection{Case Study Information}

In the following subsections, we will work on several case studies based on available information for real-life experiences from disasters such as Hurricane Katrina. Before introducing these case studies, it is important to understand the nature of Hurricane Katrina, which formed on August 23, 2005, and crossed southern Florida, causing deaths and flooding before strengthening rapidly in the Gulf of Mexico. The storm weakened as a Category 3 storm on the morning of Monday, August 29 in southeast Louisiana. Then it rapidly intensified after entering the Gulf, growing from a Category 3 hurricane to a Category 5 hurricane in just nine hours. This rapid growth was due to the storm's movement over the "unusually warm" waters of the Loop Current, which increased wind speeds (http://en.wikipedia.org/wiki/Timeline_of_Hurricane_ Katrina).

While focusing on Katrina, we will consider the New Orleans Superdome shelter that was used for sheltering purposes during Hurricanes Georges (1998), Ivan (2004), and Katrina (2005) as well. Despite previous periods of emergency use, as Katrina approached the city, officials had still not stockpiled enough generator fuel, food, and other supplies to handle the needs of the thousands of people who would be seeking refuge there. First, approximately 9,000 residents spent the night in the Superdome as Katrina came ashore with the strength of a Category 3 hurricane. According to Bennett Landreneau (http://en.wikipedia.org/wiki/Timeline_of_Hurricane_Katrina), Adjutant General for the Louisiana National Guard, the number of people taking shelter in the Superdome rose to around 20,000 as the strength of the hurricane increased and search 
and rescue teams brought more people to the Superdome from areas hit hard. On August 28, 2005, the Louisiana National Guard delivered seven truckloads of MRE's to the Superdome, enough to supply 15,000 people for three days. This was not enough for the 20,000 victims located in the Superdome. This real-life information obtained for Hurricane Katrina constitutes the basis of our case studies in the following subsections. Detailed parameter information for these case studies is given in Table 4.5.

Table 4.5 Parameter Information for Case Studies ( $\sqrt{ }$ indicates a change in the parameter whereas - means no change)

\begin{tabular}{|c|c|c|c|c|c|c|c|c|c|c|c|c|}
\hline \multirow{3}{*}{ Case Study } & \multicolumn{12}{|c|}{ Parameters } \\
\hline & \multicolumn{6}{|c|}{ Distribution } & \multicolumn{6}{|c|}{ Objective Function } \\
\hline & Mean & Variance & Kappa & $\left|\begin{array}{c}\text { Total } \\
\text { Demand }\end{array}\right|$ & $\begin{array}{c}\text { Total } \\
\text { Consumption }\end{array}$ & $\begin{array}{l}\text { Number of } \\
\text { Deliveries }\end{array}$ & $\begin{array}{l}\text { Cost of } \\
\text { Storage }\end{array}$ & $\begin{array}{l}\text { Cost of } \\
\text { Surplus }\end{array}$ & $\begin{array}{c}\text { Cost of } \\
\text { Shortage }\end{array}$ & $\begin{array}{c}\text { Cost of } \\
\text { Adjustment }\end{array}$ & $N$ & $B_{u}{ }^{\prime}$ \\
\hline $\begin{array}{l}\text { Base Case } \\
\text { (Category 3, } \\
\text { the first hit } \\
\text { by Hurricane } \\
\text { Katrina) }\end{array}$ & - & - & - & - & - & - & - & - & - & - & - & - \\
\hline $\begin{array}{l}\text { Increased } \\
\text { Strength } \\
\text { (Category 5, } \\
\text { Hurricane } \\
\text { Katrina at the } \\
\text { peak of its } \\
\text { strength) } \\
\end{array}$ & $\sqrt{ }$ & $\sqrt{ }$ & - & $\sqrt{ }$ & $\sqrt{ }$ & - & - & - & - & - & $\sqrt{ }$ & $\sqrt{ }$ \\
\hline $\begin{array}{l}\text { Changes in } \\
\text { the Mean of } \\
\text { the } \\
\text { Commodity } \\
\text { Distribution }\end{array}$ & $\sqrt{ }$ & - & - & - & - & - & - & - & - & - & - & - \\
\hline $\begin{array}{l}\text { Changes in } \\
\text { the Variance } \\
\text { of the } \\
\text { Commodity } \\
\text { Distribution }\end{array}$ & - & $\sqrt{ }$ & - & - & - & - & - & - & - & - & - & - \\
\hline $\begin{array}{l}\text { Changes in } \\
\text { the } \\
\text { Skewness } \\
\text { of the } \\
\text { Commodity } \\
\text { Distribution }\end{array}$ & $\sqrt{ }$ & $\sqrt{ }$ & - & - & - & - & - & - & - & - & - & - \\
\hline $\begin{array}{l}\text { Changes in } \\
\text { the Number } \\
\text { of Deliveries }\end{array}$ & - & - & - & - & $\sqrt{ }$ & $\sqrt{ }$ & - & - & - & - & - & - \\
\hline $\begin{array}{l}\text { Multi- } \\
\text { Suppliers/ } \\
\text { Cross } \\
\text { Shipping }\end{array}$ & - & - & - & - & - & $\sqrt{ }$ & - & $\sqrt{ }$ & $\sqrt{ }$ & - & - & - \\
\hline
\end{tabular}




\subsubsection{Multi-Commodity SHIC Case Studies}

In this subsection, we will propose a case study-based approach that can easily be incorporated into disaster relief plans so that planners can become aware of potentially serious inventory-related problems at shelters, along with their occurrence probabilities.

\subsubsection{Case Study 1 (Base Case): Change in the Severity of the Disaster}

We are first focusing on the early stages of the localized slow onset disaster Hurricane Katrina when it first hit the coastal region of the U.S. east coast with a Category 3 strength. Under this scenario, terrain is flooded, with possible disruptions in the traffic network. People leaving flood areas are gathered into shelters. We assume that 10,000 people are gathered in the New Orleans Superdome, where 2 MRE's and 1/2 medicine are assumed to be given per person per day on average (as in Lee et al., 2009). Depending on the strength of the hurricane, there are five demand levels given in ascending order; $\mathrm{u}=1$ represents the lowest demand, whereas $\mathrm{u}=5$ is the highest. Demand for MRE's increases more rapidly than the medicine as the severity of the hurricane increases. For this base case, the following values are chosen:

- $\varepsilon$ is $0.1, \zeta$ is $0.01, N$ is 100 , and $B_{u}^{(l)}$ is 1,000 for MRE's and 10,000 for medicine, respectively.

- Number of deliveries in the time interval (i.e., a day) is $n=4$.

- Amounts of initial safety stock, $m^{(1)}$ and $m^{(2)}$, are 5,000 and 20,000 units, respectively.

- $q^{+(1)}=1.2 /$ unit, $q^{+(2)}=1.0 /$ unit , and $q^{-(1)}=120 /$ unit,$q^{-(2)}=100 /$ unit . 
- $\delta^{(1)}, \delta^{(2)}, \gamma^{(1)}$ and $\gamma^{(2)}$ are calculated for each commodity separately.

- Cost of adjustment function is selected as $f(x)=2 x$.

- Costs of storage for each commodity are 5 /unit and $1 /$ unit, respectively.

- Total storage capacity is 50,000 units.

- Spaces occupied by commodities are selected as $a^{1}=2 /$ unit, $a^{2}=1 /$ unit .

- Expected total delivery and expected total consumption values are taken as

$$
\begin{gathered}
D^{1}=[2500,3000,3500,4000,4500] \quad C^{1}=[7500,8000,8500,9000,9500] \\
D^{2}=[0,4000,8000,12000,16000] \quad C^{2}=[20000,24000,28000,32000,36000]
\end{gathered} .
$$

These values are created by the 2 MRE's and $1 / 2$ medicine assumption given in (Lee et al., 2009) based on the vital supplies delivered during the emergency operations conducted by the Louisiana National Guard.

- Probabilities of the discrete supports of consumption and delivery values are

$$
p=[0.08,0.25,0.34,0.25,0.08] \text {. }
$$

Note that these supports represent demands for the consumption of commodities multiplied by their probabilities in the objective function.

Delivery and consumption values are used to calculate the mean, variance, and covariance matrix for the distribution of vital supplies. The results are given in Table 4.6. These results indicate that to satisfy the needs of victims $90 \%$ of the time, initial stock must be more than $69 \%$ of the total expected consumption of medicine. However, for MRE's, the initial stock must be more than $75 \%$ of the total expected consumption to prevent disruption $90 \%$ of the time. When the consumption is equal to the initial safety stock of MRE's, the model finds the optimal additional safety stock as 0 MRE's. 
Table 4.6 Results for the Two-Commodity Analysis of Category 3 Hurricane

\begin{tabular}{|c|c|c|c|c|c|}
\hline Demand & $\mathbf{u}=\mathbf{1}$ & $\mathbf{u = 2}$ & $\mathbf{u}=\mathbf{3}$ & $\mathbf{u = 4}$ & $\mathbf{u = 5}$ \\
\hline Initial Stock (Medicine) & 5000 & 5000 & 5000 & 5000 & 5000 \\
\hline Optimal Additional Stock (Medicine) & 250 & 500 & 750 & 1250 & 1500 \\
\hline Total Initial Stock (Medicine) & 5250 & 5500 & 5750 & 6250 & 6500 \\
\hline Total Expected Consumption (Medicine) & 7500 & 8000 & 8500 & 9000 & 9500 \\
\hline $\begin{array}{c}\text { Proportion of Initial Safety Stock to } \\
\text { Total Expected Consumption (Medicine) }\end{array}$ & $70.0 \%$ & $68.8 \%$ & $67.7 \%$ & $69.4 \%$ & $68.4 \%$ \\
\hline Initial Stock (MRE) & 20000 & 20000 & 20000 & 20000 & 20000 \\
\hline Optimal Additional Stock (MRE) & 0 & 1000 & 2000 & 4000 & 7000 \\
\hline Total Initial Stock (MRE) & 20000 & 21000 & 22000 & 24000 & 27000 \\
\hline Total Expected Consumption (MRE) & 20000 & 24000 & 28000 & 32000 & 36000 \\
\hline $\begin{array}{c}\text { Proportion of Initial Safety Stock to } \\
\text { Total Expected Consumption (MRE) }\end{array}$ & $100.0 \%$ & $87.5 \%$ & $78.6 \%$ & $75.0 \%$ & $75.0 \%$ \\
\hline Total Cost & & & 1786 & & \\
\hline
\end{tabular}

It is possible for a hurricane to intensify in strength in a short time, increasing the number of victims located in shelters and creating more demand. In 2005, Hurricane Katrina rapidly intensified after entering the Gulf, growing from Category 3 to 5; this rapid growth was due to the storm's movement over the "unusually warm" waters of the loop current, which increased wind speeds. For a Category 5 hurricane, flooding causes major damage to many structures and roadways. Massive evacuation of residential areas will be required. Therefore, we assume that 20,000 people are gathered in the New Orleans Superdome. Again, we are focusing on medicine and MRE's. Note that the daily demand for MRE's can have higher variances depending on the time-dependent impact of the disaster. Five demand scenarios are analyzed as before. All other parameters being kept the same as in the base case (other than these values: $m^{(1)}$ and $m^{(2)}$ are 500 and 2,000 units, respectively, $N$ is selected as 500, and $B_{u}^{(l)}$ is 5,000 for MRE's and 25,000 
for medicine), we expect an increase in the total delivery and consumption values at the Superdome:

$$
\begin{array}{ccc}
D^{1}=[5000,5500,6000,6500,7000] & C^{1}=[10000,10500,11000,11500,12000] \\
D^{2}=[20000,24000,28000,32000,36000] & C^{2}=[40000,44000,48000,52000,56000]
\end{array} .
$$

These values are again created by the 2 MRE's and $1 / 2$ medicine assumption given in (Lee et al., 2009) based on the vital supplies delivered during the emergency operations conducted by the Louisiana National Guard. With these values, we change the mean and variance of the distribution of the vital supplies. Especially for MRE's, the distribution is changed substantially to account for the severity of the Category 5 hurricane. As observed in Table 4.7, to satisfy the needs of the victims $90 \%$ of the time, initial stock must be more than $67 \%$ of the total consumption of medicine. That is, having more than $67 \%$ of the commodities in the inventory before the disaster will prevent disruption $90 \%$ of the time. However, for MRE's, the initial stock must be more than $73 \%$ of the total consumption. Furthermore, 20,000 victims located in a shelter can live on 2 MRE's per day, which makes the total expected consumption amount 40,000 MRE's (This is our demand $u=1$ in Table 4.7). However, the supplied amount of MRE's is 20,000 (10,000 people with 2 MRE's per day). Here, as the cost optimal result, the model gives an initial stock of 30,000 MRE's as the initial safety stock, and an additional 10,000 MRE's to be delivered afterwards.

As the strength of the hurricane increases, the demand for vital supplies increases in a short amount of time. Moreover, the demand for MRE's increases more rapidly than the demand for medicine. The model reacts to this notion by increasing the additional safety stock values more rapidly for MRE's (Figure 4.7). The most severe condition of a 
Category 5 hurricane having the largest demand requires the largest safety stocks of both commodities.

Table 4.7 Results for the Two-Commodity Analysis of Category 5 Hurricane

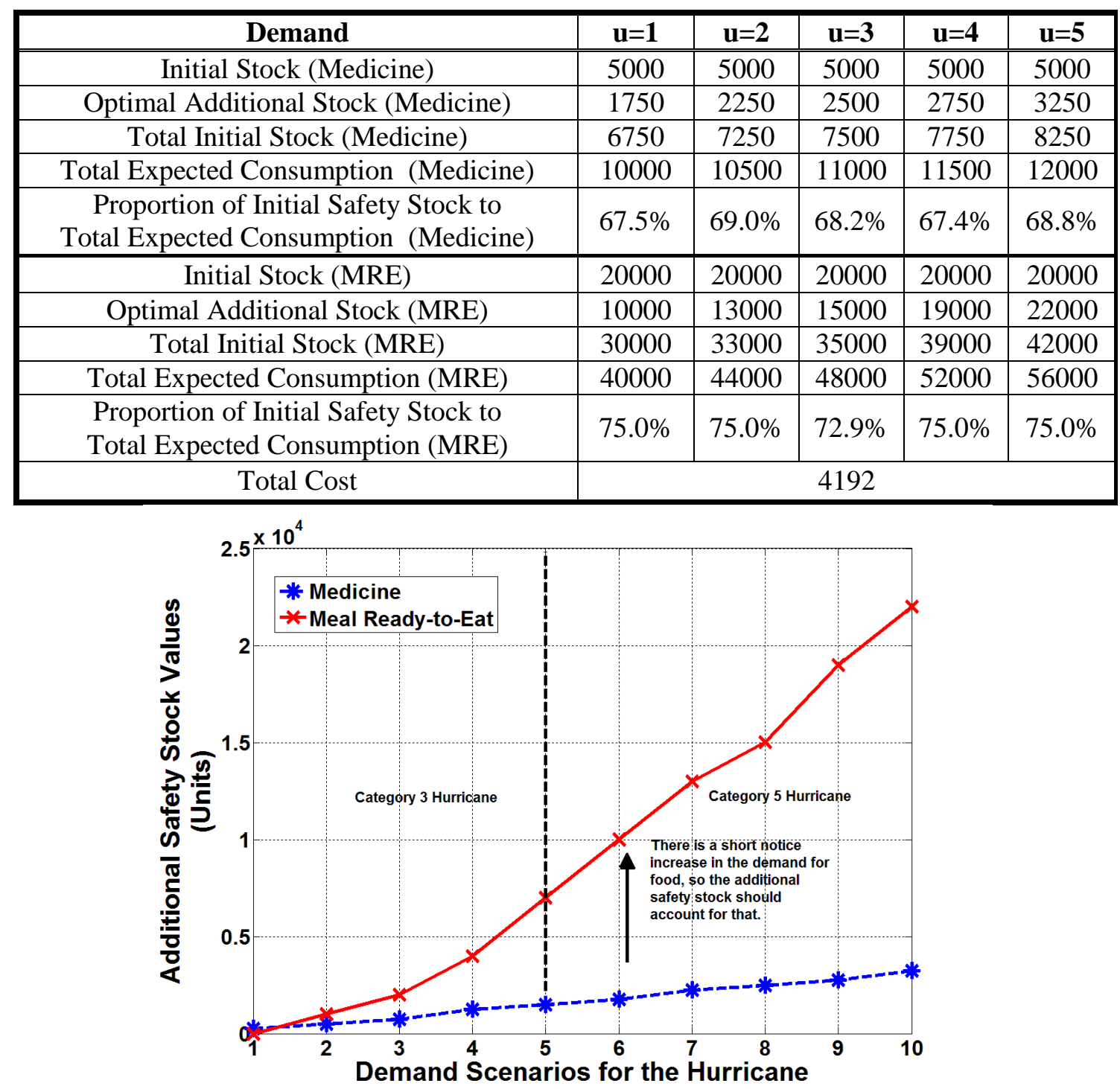

Figure 4.7 Changes in Additional Safety Stock Values versus Changes in Demand Scenarios for the Hurricane

\subsubsection{Case Study 2: Modeling Different Distributions}

The main focus of this subsection is demonstrating the importance of an efficient humanitarian inventory management system through the use of different consumption 
and delivery distributions. It is clear that a humanitarian inventory management system should be robust with respect to disruptions to minimize the impact of the severity of a particular disaster on the supply and consumption of vital commodities. Our ultimate goal here is to use realistic case studies to emphasize this simple yet very important requirement.

Changes in consumption and delivery distributions have utmost importance in determining the behavior of the model, as our model can be solved for different distributions that represent the level of unpredictability related to the level of severity of the disaster. The results of the analysis will lead to different stock levels that can be used by planners and decision makers to decide on strategies during the planning process. In our model, note that we approximate the joint distribution of the random consumption and delivery variables using an approximate multivariate normal distribution with the random variable $W_{i u}^{(l)}$ for each commodity $l=1, \ldots, r$, for $i=1, \ldots, n$, and for each support $u, u \in U$. Therefore, in this subsection, we gradually increase the skewness, mean, and standard deviation vectors of the random variable, $W_{i u}^{(l)}$, and observe the behavior of our model according to different distributions for which the relevant parameter information is given in Table 4.8 . 
Table 4.8 Parameter Selection for Different Distributions of MRE's and Medicine (Info: Category 3 Value/Category 5 Value)

\begin{tabular}{|c|c|c|c|c|}
\hline \multicolumn{5}{|c|}{$M R E^{\prime} s$} \\
\hline \multirow{6}{*}{ Mean Change } & Distribution & Mean & Standard Deviation & Skewness (Kappa) \\
\hline & Base Case Study & $250 / 8500$ & $150 / 2000$ & $0 / 0$ \\
\hline & Distribution 1 & $375 / 13600$ & $150 / 2000$ & $0 / 0$ \\
\hline & Distribution 2 & $563 / 20760$ & $150 / 2000$ & $0 / 0$ \\
\hline & Distribution 3 & $844 / 24370$ & $150 / 2000$ & $0 / 0$ \\
\hline & Distribution 4 & $1266 / 30680$ & $150 / 2000$ & $0 / 0$ \\
\hline \multirow{6}{*}{$\begin{array}{c}\text { Standard Deviation } \\
\text { Change }\end{array}$} & Distribution & Mean & Standard Deviation & Skewness (Kappa) \\
\hline & Base Case Study & $500 / 10000$ & $150 / 2000$ & $0 / 0$ \\
\hline & Distribution 1 & $500 / 10000$ & $250 / 6500$ & $0 / 0$ \\
\hline & Distribution 2 & $500 / 10000$ & $350 / 9000$ & $0 / 0$ \\
\hline & Distribution 3 & $500 / 10000$ & $450 / 12000$ & $0 / 0$ \\
\hline & Distribution 4 & $500 / 10000$ & $550 / 15000$ & $0 / 0$ \\
\hline \multirow{4}{*}{ Skewness Change } & Distribution & Mean & Standard Deviation & Skewness (Kappa) \\
\hline & Base Case Study & $500 / 10000$ & $150 / 2000$ & $-0.3 /-0.6$ \\
\hline & Distribution 1 & $1000 / 19000$ & $156 / 2150$ & $-0.6 /-1.0$ \\
\hline & Distribution 2 & $1500 / 28000$ & $162 / 2300$ & $-0.9 /-1.4$ \\
\hline \multicolumn{5}{|c|}{ MEDICINE } \\
\hline \multirow{6}{*}{ Mean Change } & Distribution & Mean & Standard Deviation & Skewness (Kappa) \\
\hline & Base Case Study & $100 / 2000$ & $50 / 750$ & $0 / 0$ \\
\hline & Distribution 1 & $150 / 3000$ & $50 / 750$ & $0 / 0$ \\
\hline & Distribution 2 & $200 / 4000$ & $50 / 750$ & $0 / 0$ \\
\hline & Distribution 3 & $250 / 5000$ & $50 / 750$ & $0 / 0$ \\
\hline & Distribution 4 & $300 / 6000$ & $50 / 750$ & $0 / 0$ \\
\hline \multirow{6}{*}{$\begin{array}{c}\text { Standard Deviation } \\
\text { Change }\end{array}$} & Distribution & Mean & Standard Deviation & Skewness (Kappa) \\
\hline & Base Case Study & $100 / 2000$ & $50 / 750$ & $0 / 0$ \\
\hline & Distribution 1 & $100 / 2000$ & $100 / 1250$ & $0 / 0$ \\
\hline & Distribution 2 & $100 / 2000$ & $150 / 2150$ & $0 / 0$ \\
\hline & Distribution 3 & $100 / 2000$ & $200 / 3050$ & $0 / 0$ \\
\hline & Distribution 4 & $100 / 2000$ & $250 / 4250$ & $0 / 0$ \\
\hline \multirow{4}{*}{ Skewness Change } & Distribution & Mean & Standard Deviation & Skewness (Kappa) \\
\hline & Base Case Study & $100 / 2000$ & $50 / 250$ & $-0.2 /-0.5$ \\
\hline & Distribution 1 & $300 / 4900$ & $63 / 980$ & $-0.4 /-0.8$ \\
\hline & Distribution 2 & $500 / 6800$ & $75 / 1250$ & $-0.6 /-0.9$ \\
\hline
\end{tabular}


The results for the mean and variance changes can be seen in Figure 4.8. As seen, the model behaves accordingly by increasing the safety stock levels with the increase in the expected consumption, or with a possible decrease in the number of deliveries. Figure 4.8 indicates that change in the variances also creates an increase in additional inventory stocks. Given the stochastic conditions in the aftermath of a disaster, this shows that our model requires more initial safety stocks to account for the high variances in consumption and delivery distribution of vital supplies. These high variances may be high for sudden onset disasters at the very beginning; therefore, the planner should be aware of the consequences of not having the optimal safety stocks in emergency inventories initially. On the other hand, they may vary with time for a slow onset disaster such as a famine, where response will require a thorough study of different consumption and delivery distributions.
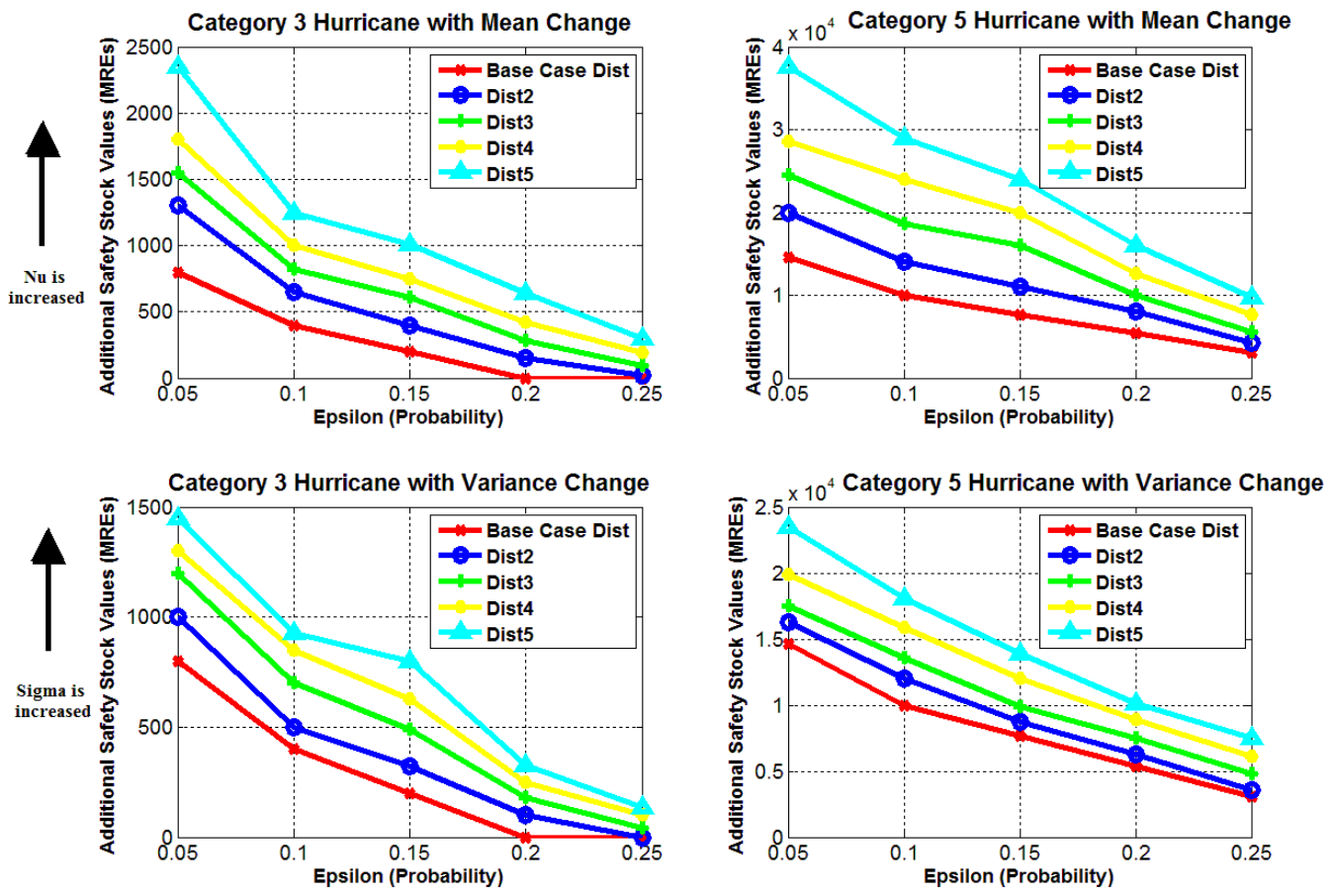

(a) 

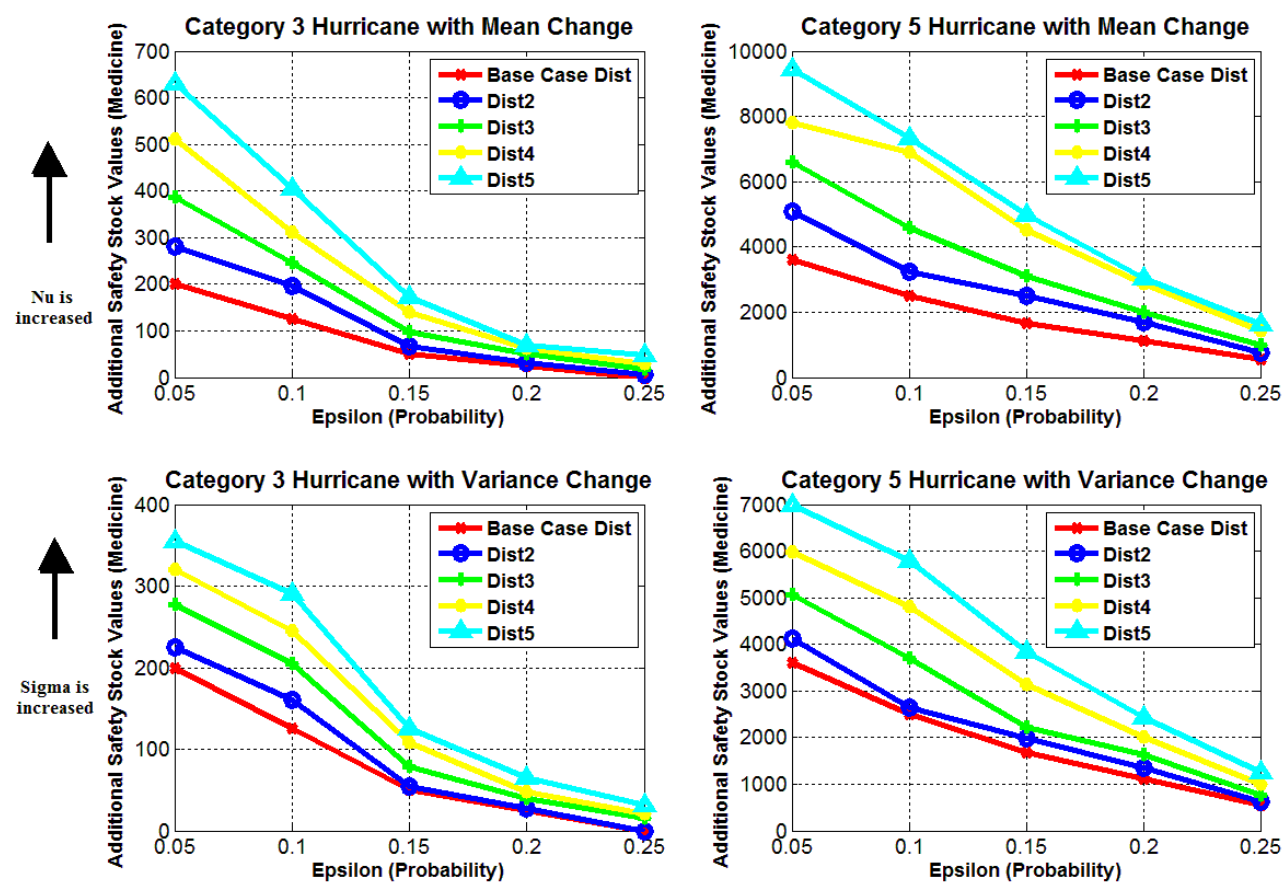

(b)

Figure 4.8 Changes in Additional Safety Stock Values for MRE's (Figure 4.8.a) for Medicine (Figure 4.8.b) with respect to Distribution Changes

Moreover, the distributions for emergency supplies can be highly skewed, especially in the early periods of a disaster. This will most likely happen for sudden onset type disasters such as the devastating Japan earthquake, where the initial need for vital supplies was enormous. To investigate this problem, we work on the skew-normal distribution given by the following equation (Azzalini and Dalla Vella, 1996):

$$
\phi(z ; \kappa)=2 \phi(z) \Phi(\kappa z)
$$

where $\phi(z)$ and $\Phi(z)$ denote the probability density and cumulative distribution functions of the normal distribution with zero mean and one as the value of the standard deviation $(N(0,1))$, respectively. The parameter $\kappa$ is the skewness parameter that regulates the skewness between $(-\infty, \infty)$, and $\kappa=0$ means the distribution of $N(0,1)$. 
Using this approach, we increase the left skewness of our distribution by decreasing $\kappa$ and observe the behavior of the model in Figure 4.9. The results show that it is appropriate to use this concept to determine the initial inventory stocks given the distributions in Table 4.8 when there is a huge demand for emergency supplies at the beginning periods of a disaster, creating the need for a left-skewed distribution. It is observed from Figure 4.9 that the additional safety stock values tend to increase as the left skewness of our distribution increases to account for the huge consumption demand coming from victims located at shelters at the early stages of the disaster. This type of analysis will definitely help support initial response operations after a disaster strikes.

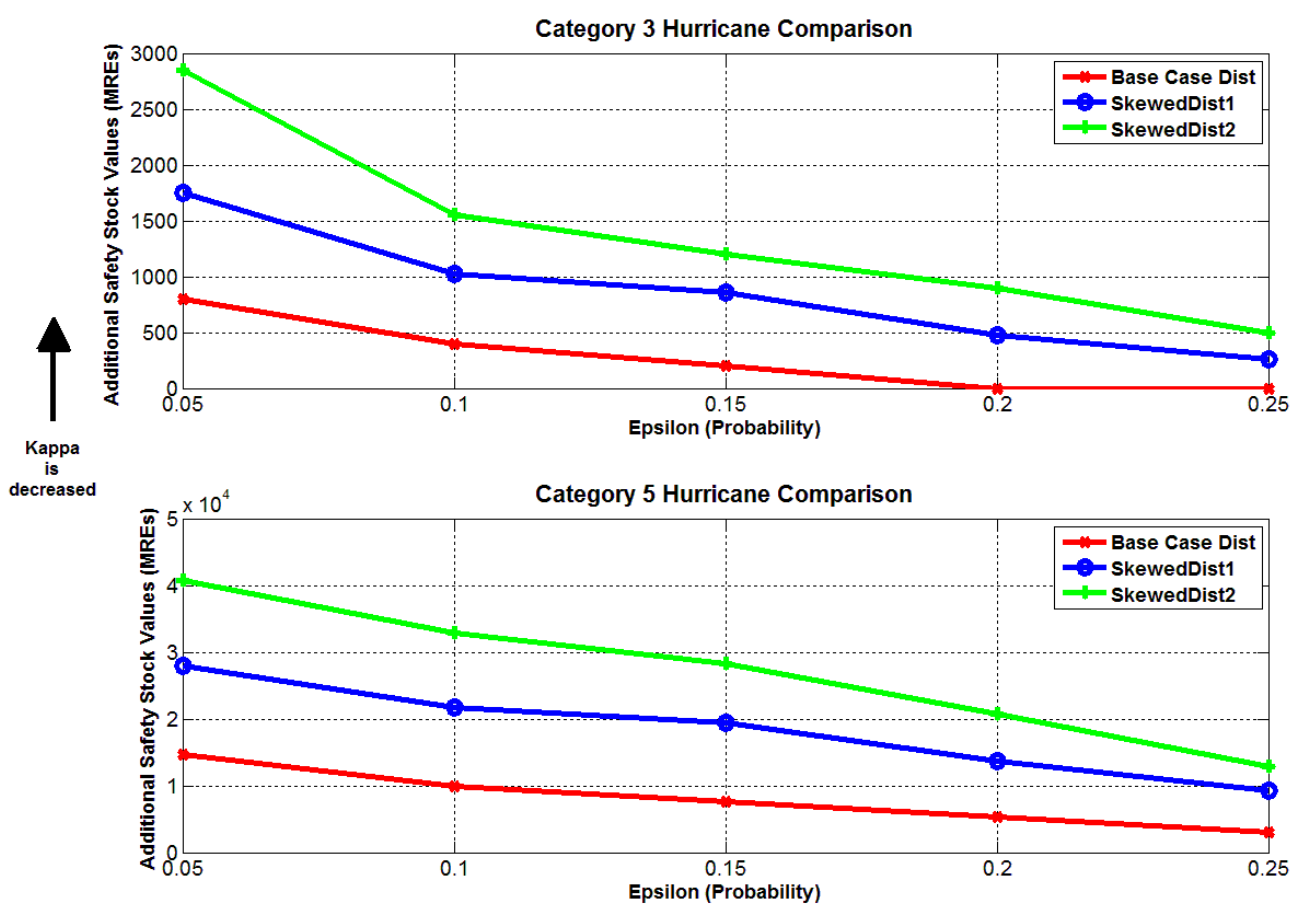

(a) 

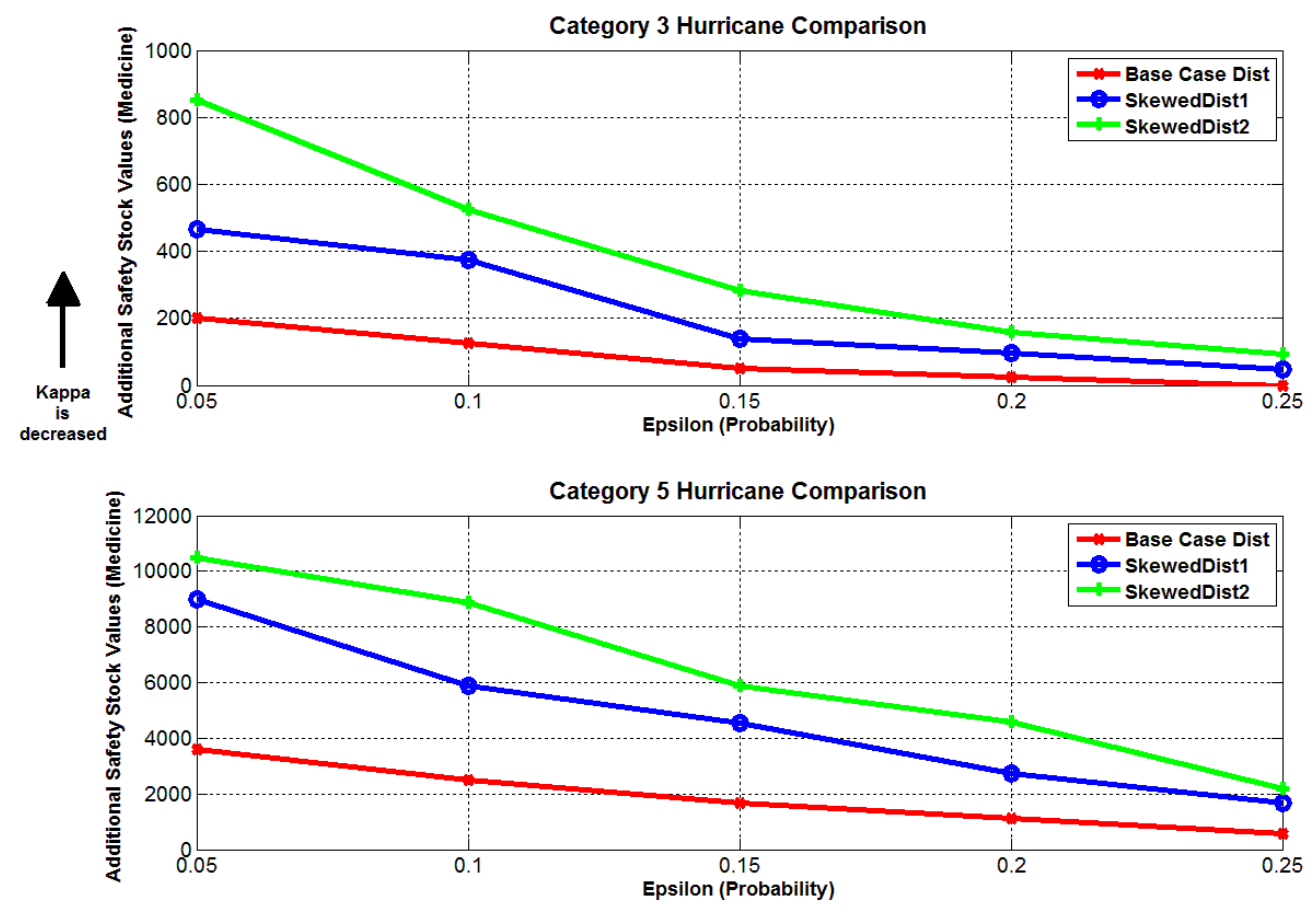

(b)

Figure 4.9 Changes in Additional Safety Stock Values for MRE's (Figure 4.9.a) for Medicine (Figure 4.9.b) with respect to Skewness Changes

\subsubsection{Case Study 3: Disruptions to the Transportation Network}

As mentioned in the previous subsection, during Katrina, the insufficient amount of supplies delivered to the Superdome caused serious problems for the 20,000 victims located in the shelter. In the aftermath of disasters, changes in the number of deliveries mainly due to the characteristics of the disaster (dispersed/localized), increased demand, reduced supply, and possible transportation system disruptions are expected. For a severe hurricane, a majority of roadways may be flooded, so a limited delivery process may be going on. This indicates the importance of determining the initial safety stocks with respect to the number of deliveries. In Figure 4.10, we start with a worst-case scenario where the vehicles cannot arrive at the shelter for the whole time period (i.e., a day), and 
gradually increase the number of deliveries to 9 (i.e., 9 trucks per day). At first, when there are no deliveries, it is necessary to stock all the emergency supplies needed as buffer. Gradually, the transportation disruptions end, and the number of vehicles serving the Superdome increases. This can be viewed as Hurricane Katrina slowing down as the wind speed decreases. As the number of deliveries increases, the additional amounts of safety stock values decrease. This is a logical behavior, as this increase leads to lower additional stock values to satisfy the probability constraint in Equation 4.9. For the Category 3 case with lower consumption needs, when the number of deliveries reaches 8 vehicles during the whole time interval, there is no need for additional safety stock for medicine and MRE's. Hence, the initial safety stock is large enough to satisfy evacuee demand for the total period. As the disaster strength increases, the initial safety stock appears to be insufficient, and a huge amount of additional safety stock is needed when the number of deliveries is limited due to damaged/congested roadways. Total cost also decreases with lower levels of additional safety stocks. This is because, if the system is highly stochastic, the probabilistic constraints are satisfied only if additional safety stock is high. This will impose higher costs. However, if the initial safety stock is too low, the problem becomes infeasible. Thus, the multi-commodity SHIC model achieves a balance between satisfying probability constraints and keeping cost low. 


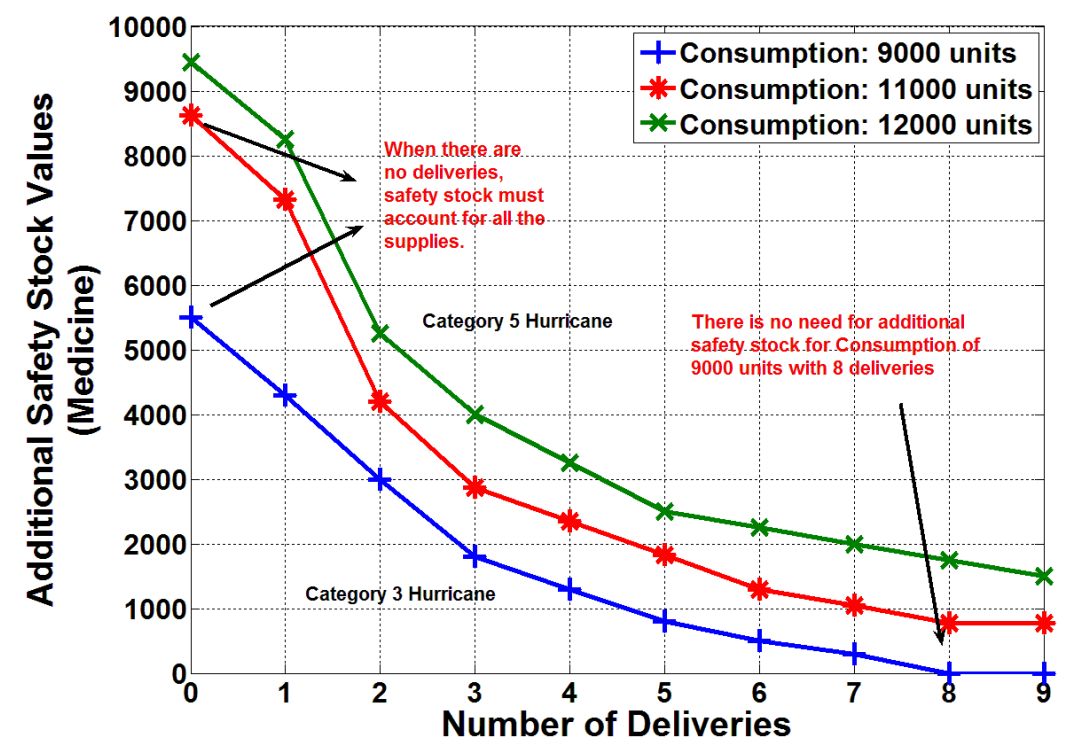

(a)

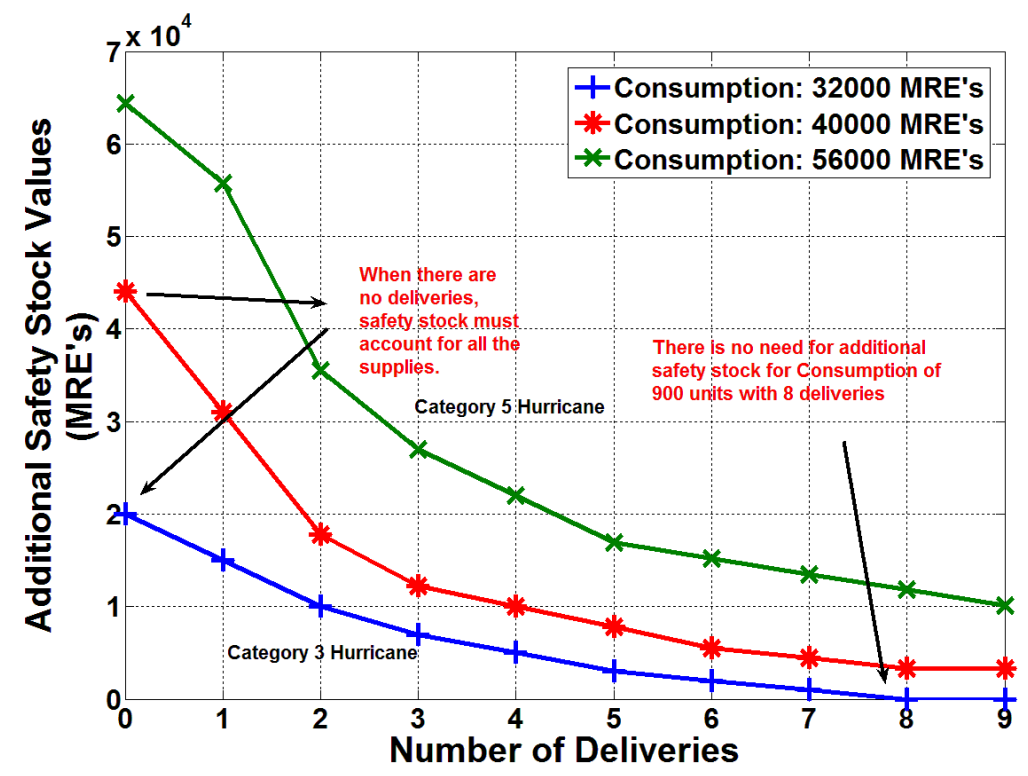

(b) 


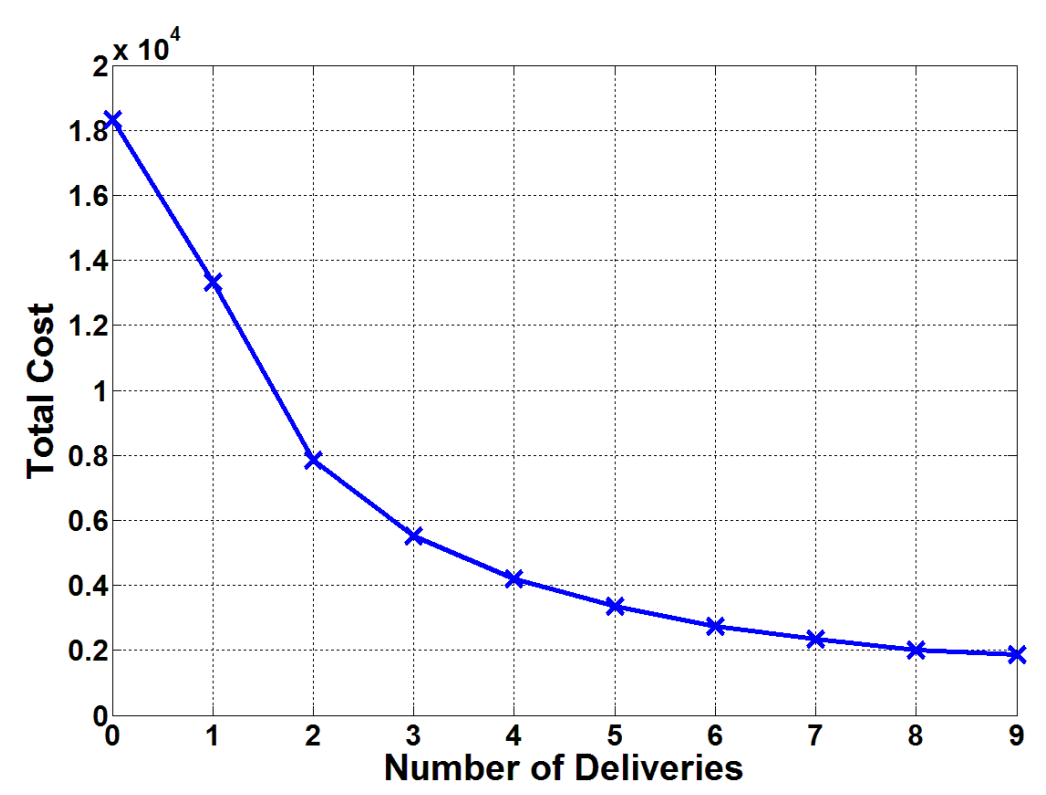

(c)

Figure 4.10 Changes in Additional Safety Stock Values for Medicine (Figure 4.10.a), for MRE's (Figure 4.10.b) and Changes in Total cost (Figure 4.10.c) for Different Number of Deliveries

\subsubsection{Case Study 4: Multi-suppliers or Cross Shipping}

In the disaster relief period, there may not be enough commodities that can be distributed to shelters from usual suppliers, or deliveries may not be possible due to a damaged traffic network (especially in the case of sudden extreme events). Therefore, to satisfy the needs of victims, emergency orders may be needed from other suppliers, which will incur more costs, not only due to monetary reasons, but also because of the time delay to supply the needs of victims located at the shelter trying to survive without a sufficient amount of vital supplies. Moreover, it is possible that some shelter inventories may experience shortages while some others experience surplus due to fluctuations in deliveries and consumption. This happened during disaster relief operations after the devastating earthquake that happened in Japan where emergency vehicles carried relief 
supplies that had been stored at the Soka Gakkai Hachinohe Culture Center in Aomori Prefecture to the Soka Gakkai Iwate Culture Center in Morioka City on March 13, 2011 (http://www.sgi.org/news/h-relief/relif2011/soka-gakkai-relief-activities-continue-innortheastern-japan.html). A similar problem occurred in the Sendai City hospital, where water supplies and food reserves were limited, and officials asked other hospitals to provide food for them; however, ground transportation was very difficult (http://www.sgi.org/news/h-relief/relif2011/soka-gakkai-relief-activities-continue-innortheastern-japan.html).

These events also indicate that rather than having deliveries come directly from the original supplier, in some cases, shelter-to-shelter deliveries may be needed between close shelters such as the New Orleans Superdome and convention center located in New Orleans. That is, if MRE's are more than enough for the victims in the convention center, and if a shortage is experienced in the Superdome, these extra MRE's can be shipped from the convention center to quickly address this shortage. Although multisupplier/cross shipping options are viable when there is a sudden notice of demand increase, it does not mean that these alternatives are always cost effective. Emergency orders are expensive, mostly in terms of time delay in satisfying the needs of victims (http://www.sgi.org/news/h-relief/relif2011/soka-gakkai-relief-activities-continue-in northeastern-japan.html). Our model reacts to this possibly more costly order from Supplier 2 based on a similar notion and increases the amount of additional safety stock instead of the commodities being delivered by Supplier 2 (Figure 4.11). This backup plan will make planners and decision makers aware of the risk involved in stocking emergency inventory commodities. 


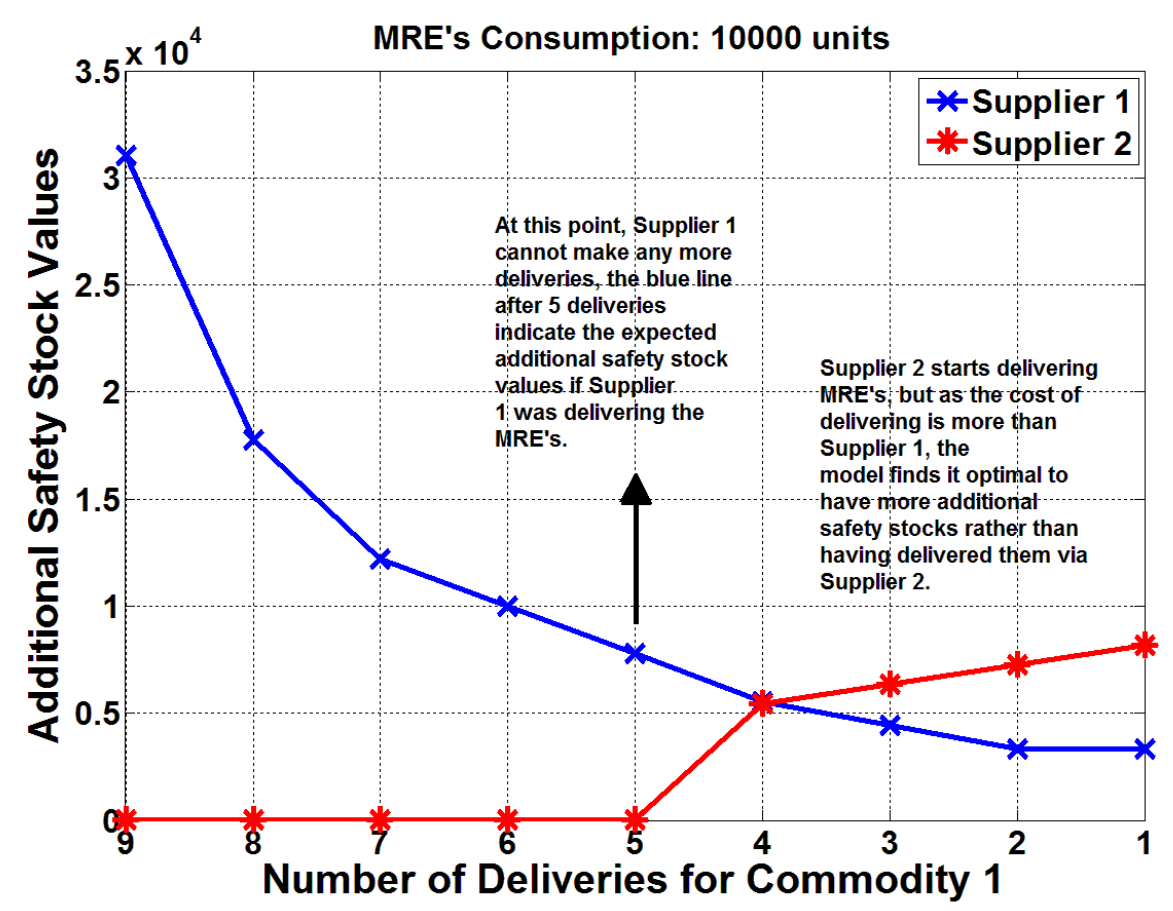

Figure 4.11 Changes in the Additional Safety Stock Values for MRE's via Multi-suppliers

\subsubsection{Case Study 5: Vital/Perishable Commodities}

This subsection includes a sensitivity analysis indicating where single-commodity and multi-commodity approaches are useful during emergency management operations. First of all, it is important to mention that some vital supplies may need extra attention during emergency relief operations. For example, if a large number of injured are brought to a shelter in the aftermath of a disaster, medicine will be immediately needed. After Katrina, in the Superdome in New Orleans, 32\% of the surveyed victims experienced injuries, with $13 \%$ of these injuries appearing to be serious (Brodie et al, 2006). Therefore, in our analysis, first, we assume that the percentage of injured people increases due to incidents caused by Category 5 hurricane conditions (the demand for medicine increases), where medicine becomes the most important commodity to be 
supplied immediately. This idea can also be used for perishable and refrigerated commodities and commodities with shelf lives, or "use by" dates. With this analysis, both emergency management of such commodities and modeling for extra inventory levels to reflect losses due to shelf life expiration and refrigeration- or power outage-related problems can be factored into the inventory control model. Members of the population with special needs can also be considered here by modeling their needs as vital commodities and therefore giving more attention to those supplies. This will allow a planner to take into account the variation in emergency needs for different segments of the population.

There is another issue in terms of transporting emergency supplies, which mostly occurs for no-notice disasters where delivery using the transportation network is almost impossible at the very beginning of the disaster. As Barbarosoglu et al. (2002) stated, using helicopters for delivering supplies during disasters might be necessary depending on the level of disruption of the transportation network. After the recent earthquake in Japan, most of the roadway network was unavailable or only partially available for delivery operations. Therefore, helicopter transportation became a key issue. For example, CH-46E Sea Knight helicopters from the Marine Medium Helicopter Squadron 265 (HMM-265), Marine Aircraft Group 36, 1st Marine Air Wing, III Marine Expeditionary Force delivered supplies from the Naval Air Facility in Atsugi, Japan, to earthquake and tsunami victims located in shelters in the north of Sendai, Japan (http://www.dvidshub.net/tags/video/hmm-265/page/3). Immediately after the earthquake, two Marine helicopters delivered 1,500 pounds of rice and bread as emergency supplies to shelters located in the hardest hit area in Japan, Shioishi City in 
the Miyagi Prefecture (http://abcnews.go.com/International/japan-earthquake-usmobilizes-humanitarian-military-relief/story?id=13124482). Meanwhile, five Air Force helicopters and crews from rescue squadrons were en route to an air base near Tokyo to support search and rescue missions. This kind of emergency delivery using helicopters can also be calculated via the vital commodity approach presented in this subsection.

The analysis is conducted with a single-commodity approach at first. Then the results are compared with the two-commodity analysis performed as before with the medicine and MRE's together. As observed, the total cost for this analysis is larger than the single-commodity analysis cost (Table 4.9). This is because we are focusing on a vital single commodity rather than two commodities, and the cost is substantially high relative to a less important commodity, as the shortage costs for medicine are higher. Due to these high costs, the model does not allow a high safety stock for medicine, and the resulting total base stock values are approximately $10 \%$ lower than in the two-commodity case.

The decision of which method to use (joint constraints or individual constraints based on single- or multi-commodity analyses) depends on the priorities of the disaster planning process, where the planner should be careful about the requirements of the decision-making process. If the focus is on a single commodity and perishability is our main concern, or if other types of costly transportation such as deliveries by helicopter are needed due to a damaged roadway network, it is useful to conduct a singlecommodity analysis. In this case, the cost becomes higher due to stocking requirements and shortage possibilities. If those concerns are minimal, and if we are just concerned with quantities of safety stocks in emergency inventories, a multi-commodity analysis is required. During emergency conditions in the aftermath of a disaster, transportation of 
joint resources, i.e., food and water, is also a major concern, as emergency vehicles supplying shelters may be limited due to disruptions in the transportation network and unavailability of personnel and vehicles. Moreover, relationships may exist between product demand streams such as food items or medical supplies, leading to substitution possibilities during a shortage. In these cases, a multi-commodity analysis would be logical.

Table 4.9 Comparison for Single and Two-Commodity Analysis for Medicine

\begin{tabular}{|c|c|c|c|c|c|}
\hline \multicolumn{7}{|c|}{ Single-Commodity Analysis } \\
\hline Demand & $\mathbf{u}=\mathbf{1}$ & $\mathbf{u}=\mathbf{2}$ & $\mathbf{u}=\mathbf{3}$ & $\mathbf{u = 4}$ & $\mathbf{u = 5}$ \\
\hline Initial Stock & 5000 & 5000 & 5000 & 5000 & 5000 \\
\hline Optimal Additional Stock & 1400 & 1550 & 1700 & 1850 & 1975 \\
\hline Total Initial Stock & 6400 & 6550 & 6700 & 6850 & 6975 \\
\hline Total Expected Consumption & 10000 & 10500 & 11000 & 11500 & 12000 \\
\hline $\begin{array}{c}\text { Proportion of Initial Safety Stock to } \\
\text { Total Expected Consumption }\end{array}$ & $64.0 \%$ & $62.4 \%$ & $60.9 \%$ & $59.6 \%$ & $58.1 \%$ \\
\hline Total Cost & & & 2974 & & \\
\hline \multicolumn{2}{|c|}{ Two-Commodity Analysis } & & & \\
\hline Demand & $\mathbf{u = 1}$ & $\mathbf{u = 2}$ & $\mathbf{u = 3}$ & $\mathbf{u = 4}$ & $\mathbf{u = 5}$ \\
\hline Initial Stock & 5000 & 5000 & 5000 & 5000 & 5000 \\
\hline Totimal Additional Stock & 1750 & 2250 & 2500 & 2750 & 3250 \\
\hline Total Initial Stock & 6750 & 7250 & 7500 & 7750 & 8250 \\
\hline $\begin{array}{c}\text { Potal Expected Consumption } \\
\text { Total Expected Consumption }\end{array}$ & 10000 & 10500 & 11000 & 11500 & 12000 \\
\hline Total Cost & $67.5 \%$ & $69.0 \%$ & $68.2 \%$ & $67.4 \%$ & $68.8 \%$ \\
\hline
\end{tabular}




\subsubsection{Discussion}

Due to the highly probabilistic nature of demands and supplies in the aftermath of disasters, probabilistic inventory control models are better suited to the development of realistic plans. However, there are not many practical models in the literature for which the convergence properties can be analytically shown due to modeling and solution complexities. In this chapter, we propose to use a humanitarian inventory control methodology as the base model for our case studies, which has been proven to reach optimality (Prékopa, 2006). Unlike previous approaches in the literature, this case studybased model is designed to successfully address the critical and strategic factors associated with humanitarian relief operations to ensure the continuity of unpredictable stochastic delivery and consumption processes. Moreover, the flexibility of the proposed inventory management and control methodology allows the application of our mathematical model to any extreme event, accounting for the flexibility measures of humanitarian relief operations, namely the ability to change the output levels of products and the flexibility of delivery times, defined by Beamon and Balcik (2008).

Results of the proposed multi-commodity stochastic humanitarian inventory control model, when applied to various case studies, is encouraging in the sense that it provides a basis for the realistic analysis of risk involved in planning for emergency stock levels. The practical and easily applicable Prékopa-Vizvari-Badics algorithm is used to solve the problem so that the case study-based approach can be incorporated into disaster relief plans, and planners can become aware of various potentials for serious inventoryrelated problems that can occur at shelters. By concentrating on high-risk scenarios, a planner can take recovery actions to ensure the best allocation strategy for vital resources. 


\subsection{Summary of Key Findings}

Several realistic case studies built using information available from past disasters are studied using the model, addressing the following important issues:

- Change in the severity of the disaster,

- Disruptions in the transportation network,

- Emergency orders/cross shipping possibilities,

- Change in consumption and delivery distributions, and skewness.

The findings of this chapter based on the case studies can be listed as follows:

- An increase in the severity of a disaster causes an increase in the consumption levels of vital supplies in shelters. Therefore, as the demand for a given commodity increases, the corresponding initial safety stock values increase too.

- The analysis results indicate that the greater the number of deliveries during the emergency relief period is the less additional safety stock values are required. Thus, if transportation/supply systems are robust enough to support a higher level of deliveries, the authorities can afford to maintain lower levels of safety stocks. This will reduce the cost of emergency preparedness without compromising the wellness of evacuees.

- Multi-suppliers/cross shipping and transportation of vital and perishable commodities require extra attention while deciding on safety stock levels.

- The risk and uncertainty involved during emergency relief operations directly influence consumption and delivery distributions, which may have high means or 
variance, or may be highly skewed. Therefore, different consumption and delivery patterns and high skewness should be taken into account when performing a realistic emergency inventory management analysis.

After presenting and discussing the offline planning model, namely SHIC, we will introduce the proposed online control methodologies within our emergency inventory management framework in the next chapter. 


\section{CHAPTER 5. ONLINE CONTROL \& INVENTORY MANAGEMENT}

There is a crucial need for the development and analysis of a humanitarian inventory management model such as the proposed SHIC model in the previous chapter prior to the occurrence of a natural or man-made catastrophe. However, an emergency inventory management system should also include an online operational strategy to minimize the impacts of unforeseen disruptions (possibly during a slow onset disaster where predictions may not be accurate), or at least to address the problem at hand as quickly as possible (especially for a sudden onset disaster where fast response has utmost importance). The real-time management of preparedness activities will reveal the actual performance of the response, recovery, and mitigation processes after the disaster. Therefore, the problem is to be able to have an online inventory control methodology within our framework. As stated by Pao et al. (1992), determining the controllers for nonlinear systems is extremely difficult, even in deterministic settings where the equations that govern system dynamics are fully known. Similarly, within SHIC, our complex inventory management model, it is impossible to determine the control law needed using existing adaptive control procedures. This provides the motivation for developing an online inventory control procedure that does not require a model or that requires minimal information for the underlying inventory management system, which will be introduced in the next sections starting with an intuitive explanation of the model. 


\subsection{Model 1: Functional Approximator Approach}

Under perfect conditions, which clearly do not exist during or after disasters, the inventory stock levels obtained via the SHIC model can be used repeatedly to supply the basic needs of disaster victims located in shelters. One of the major challenges here is that it is difficult to control and regulate the system given the stochasticity in the nature of underlying supply and consumption processes. When the system parameters (not the structure of the model) are unknown, feedback control procedures can be used with a control law estimating these parameters using data. However, these methods are limited, as they need some sort of system equations for the model. For complex disaster processes, on the other hand, these closed-form equations describing the system dynamics are generally unknown, making it impossible to determine the control law. For this purpose, we propose to use a function approximator (FA) to deal with noisy data and to obtain an acceptable control methodology without having to explicitly model the process dynamics for our emergency relief system.

The overall proposed control methodology can be seen in Figure 5.1 where the model is comprised of two distinct phases: offline FA training process in the presence of noise using SHIC model and online model that uses FA to calculate the output inventory levels. The input data for the training of FA are generated by the SHIC model using the probabilistic consumption and delivery distributions of emergency commodities. Then white noise is introduced into the system to better simulate the extreme complexity expected under real-world emergency conditions. 


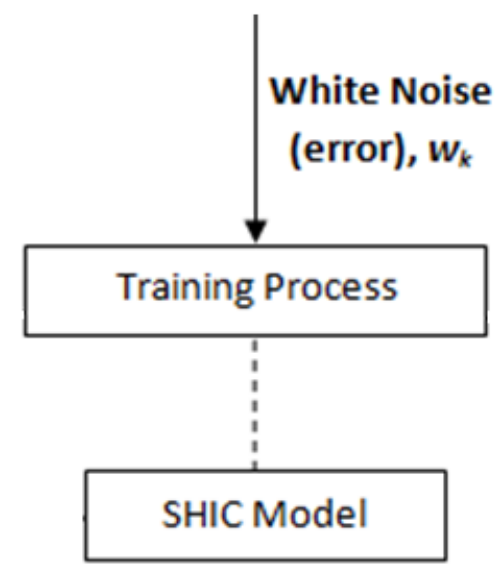

(a)

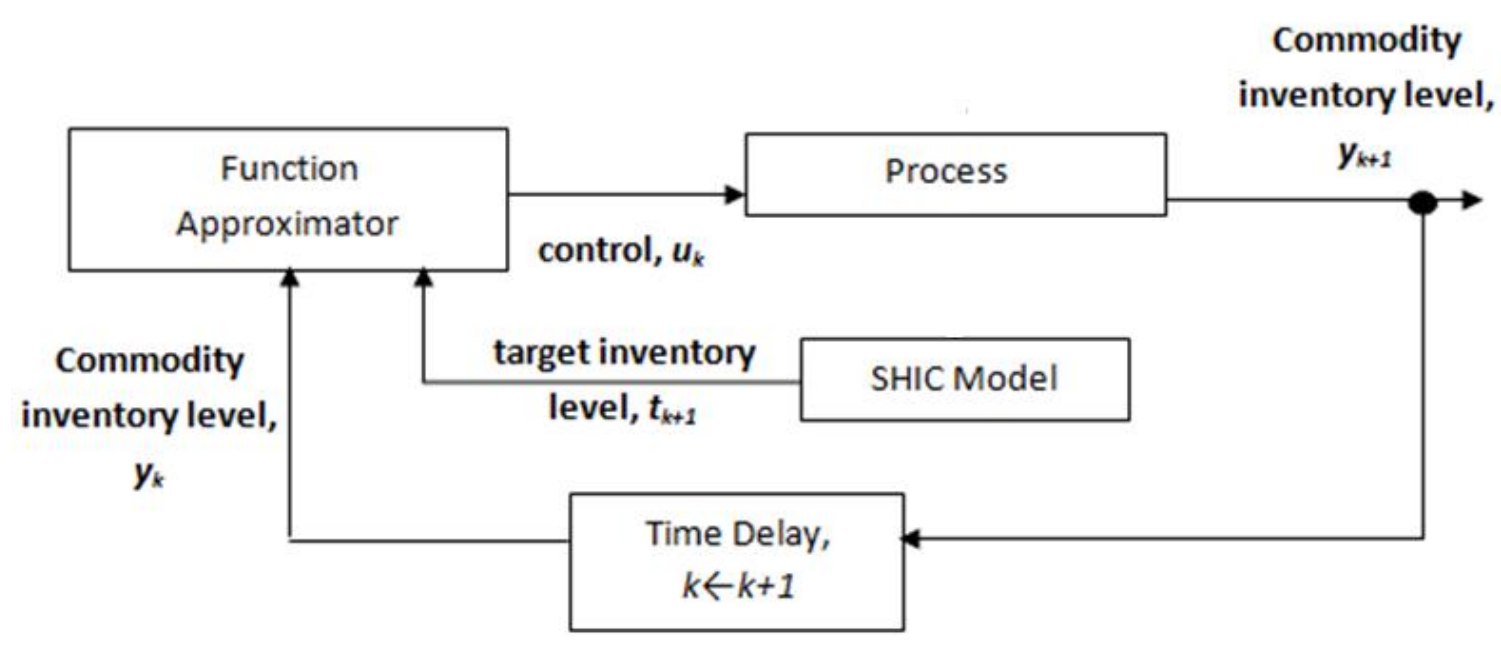

(b)

Figure 5.1 Model-free Feedback Control Methodology: (5.1.a) Offline Training of the Function Approximator using SHIC Model (5.1.b) Online Control Model based on the Function Approximator and SHIC Model

In our FA-based model, $y_{k+1}$ are the output inventory levels of emergency commodities, where $k$ represents the time step. We have three basic goals in our model:

- to adapt or train the function approximator with the random consumption and delivery distributions of the multi-commodity SHIC model, which is done offline. 
- to approximate the control vector $u_{k}$ using the time-invariant functional approximator, and

- to reach the desired target inventory levels by penalizing the deviation between the output inventory values, $y_{k+1}$, and the target inventory values.

We are going to introduce the proposed function approximator that can achieve these goals in the next subsections, first introducing an intuitive explanation.

\subsubsection{Motivation and Intuitive Explanation}

As mentioned in the previous chapter, safety stock values calculated via SHIC model can be used to provide the vital needs of disaster victims under perfect conditions that do not exist in the aftermath of the disasters. Here, the main problem is the uncertainty involved in the disaster relief operations which usually happen due to damaged roadways, demand fluctuations and lost inventories. However, it is also possible that information regarding the disaster supply chain is not available or transparent enough to deduct logical conclusions for inventory decisions. This type of information loss is possibly due to lost or disrupted communications infrastructure. As this will affect the online tracking process (RFID) and create noisy data, our online control model based on functional approximation should be able to deal with these noisy measurements in the aftermath of a disaster. With this motivation, we would like to answer the following intuitive questions using the functional approximation:

- Can we use a practical functional approximator to represent real-life conditions without having the need for solving the SHIC problem at every time step? 
- Can this functional approximator respond efficiently to the stochasticity involved within the disaster conditions efficient enough by reaching the optimal target inventory stock levels in a reasonable amount of time?

In the next subsection, we will give the basic assumptions for the online model on real-life disaster conditions.

\subsubsection{Basic Assumptions}

The modeling assumptions for the functional approximator on representing reallife conditions include the following:

- We assume that SHIC model is representing what is expected to happen in the real world and use it for simulating the consumption and delivery distributions of vital supplies to feed the functional approximator as sufficient real-life data is not available for training purposes.

- White Gaussian noise is assumed to be representing the stochasticity in the aftermath of a disaster used to better simulate the extreme complexity expected under real-world emergency conditions. That is, the model considers this uncertainty as adding random noise to the commodity distribution, creating normally distributed random deviations from the average pattern.

- The objective function is assumed to be an error tracking function where the difference between target inventory levels obtained via SHIC model and the output inventory levels obtained from the functional approximator is compared for minimization process. 


\subsubsection{Functional Approximator}

In our model, we propose to use a neural network as the function approximator (FA) that will be trained with the data obtained from the multi-commodity SHIC model (SHIC is serving as a simulator for the FA). Then this function approximator will be used to approximate the control vector that would normally be calculated for systems for which a closed-form control law cannot be obtained. Neural networks have been used to simulate this type of inventory control and supply chain model via the training process in several studies such as Ezziane et al. (1993) and Bansal et al. (1998).

\subsubsection{Offline Training Procedure}

To obtain a relevant model to obtain our data, we focus on the following idea: The inventory level at any time step should be equal to the inventory level from the previous time step plus the consumption and delivery amounts within the current time step, including noise. (The conceptual model can simply be written as $y_{k+1}=y_{k}+c_{k}-d_{k}+w_{k}$

for illustration, where $y_{k+1}, y_{k}$ represent inventory levels, $c_{k}$ and $d_{k}$ are consumption and deliveries, and $w_{k}$ is the stochastic noise for time step $k$.) By employing this concept on the SHIC model, we propose the following equation to create training data for the FA where white Gaussian noise (error) is introduced into the system at every time step $k$ :

$$
y_{k+1}=\Lambda y_{k}+\Upsilon W_{k}+E w_{k}
$$

where $\Lambda$ : noise matrix for the current inventory level, where any element of $\Lambda$ is a normally distributed random noise selected between $[-10,10]$ chosen suitable for the data set. 
$\Upsilon$ : noise matrix for $W_{k}$, which we select as a diagonal matrix, with the diagonal elements equal to 1 (however, it can always be changed to create noisy observations from the SHIC model).

$W_{k}$ : Multi-commodity SHIC vector representing the stochastic "consumptiondelivery" distribution at time step $k$ (a randomly selected element from Equation $(4.5))$.

$E$ : multiplication matrix, where any element of $E$ is $\left\|y_{k}\right\|$.

$\left\|y_{k}\right\|:$ the Euclidean norm of the inventory level $y_{k}$ at time step $k$.

$w_{k}:$ an independent scalar of Bernoulli \pm 0.5 process.

This type of model is interesting, as the noise enters the system in a multiplicative and very unstable way, rather than simply adding the noise to the current inventory levels. Note that these data do contain the consumption and delivery distributions from the SHIC model, and the output inventory levels are not necessarily optimal results.

The optimization problem based on the trained neural network will be introduced later in this section, but first, we will focus on the description of the proposed neural network. Neural networks can be used to deal with the problems of controlling and regulating stochastic systems with unknown nonlinear dynamic, as they can be used to approximate the underlying controlling system without the need to construct a separate model for the unknown process dynamics. Neural networks have been known to be exceptionally successful in learning complex behavior from observations. These issues generate the basis for considering the use of neural networks for the feedback control problem given in this subsection. Neural networks have been used for similar purposes 
for different problems in transportation and traffic engineering fields. Examples of neural network studies include their applications to feedback-based ramp metering strategies (Zhang and Ritchie, 1997; Bao and Lin, 2009) and traffic control systems (Shen and Kong, 2009; Peng et al., 2011).

The multi-layer feed forward neural network model considered in this study has a fixed number of layers and nodes (Figure 5.2). The variation of the network comes from the updates of the underlying parameters at every iteration. These underlying parameters for the neural network are the connection weights between the inputs, hidden layers, and outputs, and the bias values included in the hidden layers and outputs. Another important issue with this neural network is that it has no information about the analytical structure of the SHIC model generating the measurements (no prior information about the structure of the system).

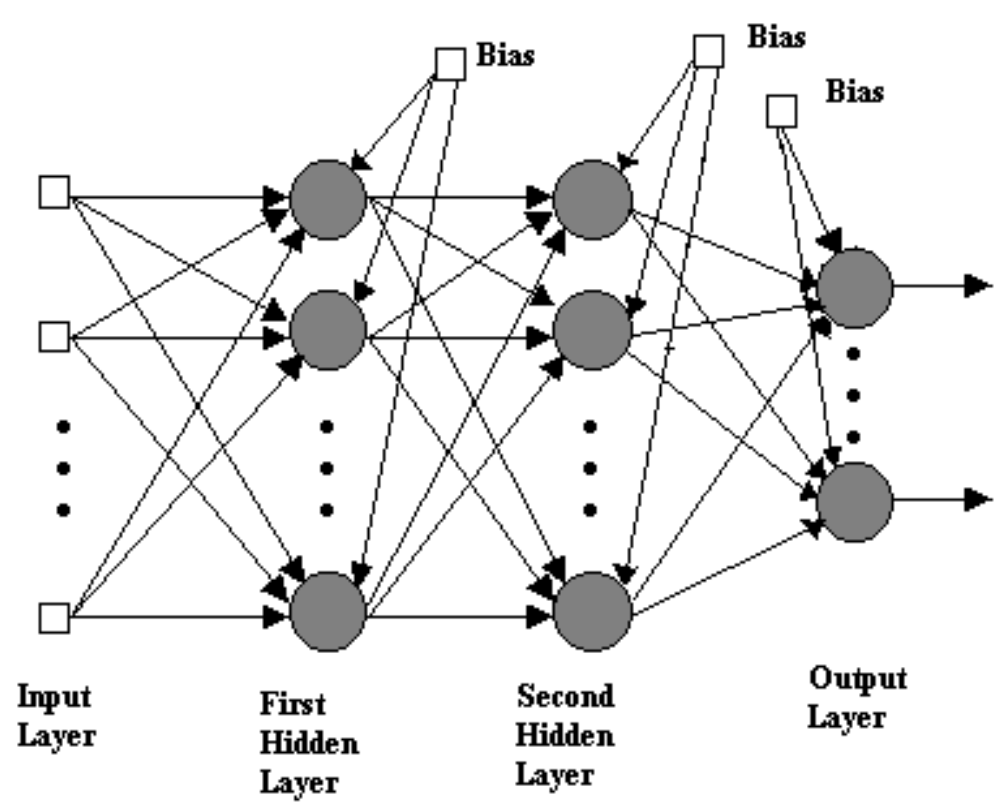

Figure 5.2 Multi-layer Feed Forward Neural Network 


\subsubsection{Online Functional Approximator-based Methodology}

As stated by Spall (1998), finding the optimal control strategy for the neural network at time step $k$ is equivalent to minimizing the following error function:

$$
\min L_{k}\left(\theta_{k}\right)
$$

where $\theta_{k} \in R^{p}$ is a $p$-dimensional vector of the weights of the neural network and $p$ is not a function of $k$ and represents the number of weights to be optimized. We use the optimal values of $\theta_{k}$ to obtain the control vector $u_{k}$, which will be used to calculate the optimal values of outputs. The objective function used is based on the expected value $(E[\bullet])$ of the quadratic tracking error loss function:

$$
L_{k}\left(\theta_{k}\right)=E\left[\left(y_{k+1}-t_{k+1}\right)^{T} A_{k}\left(y_{k+1}-t_{k+1}\right)+u_{k}^{T} B u_{k}\right]
$$

where $A_{k}$ and $B_{k}$ are positive semi-definite matrices indicating the relative weight of the deviations from the target values $t_{k+1}$ and the cost associated with larger values of the controls. Therefore, our unconstrained nonlinear optimization problem becomes:

$$
\min L_{k}\left(\theta_{k}\right)=E\left[\left(y_{k+1}-t_{k+1}\right)^{T} A_{k}\left(y_{k+1}-t_{k+1}\right)+u_{k}^{T} B u_{k}\right]
$$

where $\theta_{k} \in R^{p}$ is a $p$-dimensional vector of the weights and $p$ is not a function of $k$.

Intuitively, the aim in minimizing this objective function is to find the optimal control vector which will be used to calculate the optimal inventory stock values. As one can observe from Equation (5.4), there is no explicit control law available and therefore there are no closed form equations to solve the problem explicitly which clearly complicates the solution process. For example, if the problem was linear time-invariant, 
the solution would be easier through the use of exact mathematical algorithms. However, in the absence of such linearity and in the presence of time dependence, we need to have other means of approaching the problem such as heuristics. Methods presented here represent heuristic approaches to solve this problem practically. It is significant to note that, in our neural network, associated with the control vector $u_{k}$ for the controller, there is an underlying connection weight vector $\theta_{k}$, which is to be trained for optimality toward the optimal inventory level values. Following the training process, to solve the problem of finding the optimal control vector using the neural network based on the unconstrained nonlinear optimization problem given in Equation (5.4), Levenberg-Marquardt (LM), a built-in algorithm within MATLAB, will be used (Levenberg, 1944; Marquardt, 1963).

The algorithm for the overall functional approximation procedure using the neural networks is presented as follows:

Step 0. The SHIC model is assumed to be representing what is expected to happen in the real world. It will produce the consumption and delivery distributions in terms of $W_{k}$.

Step 1. Given the inputs $\left\{y_{k}, W_{k}, w_{k}\right\}$ at time step $k$, generate a vector of timedependent outputs $\left\{y_{k+1}\right\}$ based on Equation (5.1).

Step 2. Use these inputs and outputs to train the neural network.

Step 3. Go to Step 1 and create new outputs $N N\left(y_{k}\right)=\left\{y_{k+1}\right\}^{N N}$ based on the new inputs and trained neural network. 
Step 4. Calculate the target inventory level from the SHIC model as $\operatorname{SHIC}\left(y_{k}\right)=\left\{y_{k+1}\right\}^{\text {SHIC }}$.

Step 5. Solve Equation (5.4), where $\left\{y_{k+1}\right\}^{\text {SHIC }}=t_{k+1}$ and $\left\{y_{k+1}\right\}^{N N}=y_{k+1}$ using the LM algorithm.

Step 6. Repeat until the convergence criteria are achieved based on the difference of two consecutive objective functions: $L_{k+1}\left(\theta_{k+1}\right)-L_{k}\left(\theta_{k}\right) \leq \varepsilon$, where $\varepsilon$ is a predetermined small performance parameter.

Now that we have given the mathematical basis for the neural network-based method, we will introduce an efficient stochastic approximation method in the following subsections that can efficiently solve the unconstrained minimization problem given in Equation (5.4), and we will compare it with the neural network-based approach.

\subsubsection{Simultaneous Perturbation Stochastic Approximation (SPSA) Method}

In Equation (5.4), the minimization of $L_{k}\left(\theta_{k}\right)$ actually requires, for each $k$, a minimizing solution $\theta_{k}^{*}$ to the following equality:

$$
g_{k}\left(\theta_{k}\right)=\frac{\partial L_{k}}{\partial \theta_{k}}=\frac{\partial u_{k}^{T}}{\partial \theta_{k}} \frac{\partial L_{k}}{\partial u_{k}}=0
$$

As $\frac{\partial L_{k}}{\partial u_{k}}$ cannot be computed, it is not generally possible to calculate $g_{k}\left(\theta_{k}\right)$, and the standard gradient descent-type algorithms cannot solve our problem. Hence, we consider the stochastic approximation algorithm that has the standard recursive form of

$$
\widehat{\theta}_{k}=\widehat{\theta}_{k-1}-a_{k} g_{k}\left(\hat{\theta}_{k-1}\right)
$$


where the gain sequence $\left\{a_{k}\right\}$ satisfies the well-known conditions (Robbins and Monro, 1951):

$$
\begin{aligned}
& \sum_{k=1}^{\infty} a_{k}=\infty \\
& \sum_{k=1}^{\infty} a_{k}^{2}<\infty
\end{aligned}
$$

Here, $g_{k}\left(\hat{\theta}_{k}\right)$ is the estimate of the gradient $g_{k}\left(\theta_{k}\right)=\frac{\partial L_{k}}{\partial \theta_{k}}$, and $\hat{\theta}_{k}$ is the estimate of the weight vector calculated at any iteration solving the unconstrained optimization problem.

We propose the simultaneous perturbation stochastic approximation (SPSA) method, first proposed by Spall (1992), to find the estimate of the gradient given in Equation (5.6), as SPSA only requires two measurements of the system rather than its full functional form to calculate the gradient direction. That is, if there is a deviation from target levels, we use the online SPSA method to restore the inventory levels to optimal values. This type of approximation is applied by Spall and Cristion (1997) and Spall (1998) to wastewater treatment-related problems.

In our model, the SPSA algorithm uses the form in Equation (5.6), where $\widehat{g}_{k}\left(\widehat{\theta}_{k}\right)$ represents the simultaneous perturbation approximation to $g_{k}\left(\widehat{\theta}_{k}\right)$. The ith component of $\widehat{g}_{k}\left(\widehat{\theta}_{k}\right), i=1, \ldots, p$, is calculated as:

$$
\widehat{g}_{k i}\left(\widehat{\theta}_{k}\right)=\frac{\widehat{L}_{k}^{(+)}-\widehat{L}_{k}^{(-)}}{2 c_{k} \Delta_{k i}}
$$

Here,

- $\widehat{L}_{k}^{( \pm)}$: estimated values of $L_{k}\left(\widehat{\theta}_{k-1} \pm c_{k} \Delta_{k}\right)$ using the observed $y_{k+1}^{( \pm)}$and $t_{k}$. 
- $\hat{\theta}_{k}$ : weight vector used to approximate $u_{k}^{( \pm)}$in the neural network, where $\hat{\theta}_{k}=\hat{\theta}_{k-1} \pm c_{k} \Delta_{k}$, and $\Delta_{k}=\left(\Delta_{k 1}, \Delta_{k 2}, \ldots, \Delta_{k p}\right)^{T}$, with the $\left\{\Delta_{k i}\right\}$ independent bounded, symmetrically distributed random variable elements, for each $\forall k, i$, that are identically distributed at each $k$, with the $E\left(\Delta_{k i}^{-2}\right)$ uniformly bounded, for each $\forall k, i .\left\{\Delta_{k i}\right\}$ cannot be uniform or normal.

- $y_{k+1}^{( \pm)}$: inventory level state values based on the model.

- $\left\{a_{k}\right\}$ and $\left\{c_{k}\right\}$ are called gain sequences, and they are sequences of positive numbers. In a setting where the dynamics and objective function are changing, it is best to pick constant coefficients as $a_{k}=a, c_{k}=c \forall k$ in order to satisfy the traceability of the time-varying solution $\theta_{k}^{*}$ (Spall, 1998).

The power of SPSA comes from the fact that only two inventory level measurements are needed at any iteration to estimate the gradient direction. This is in contrast to other standard finite difference stochastic approximation methods, which would need $2 p$ measurements by definition to calculate the optimal $\theta_{k}^{*}$ vector with a dimension $p$. Moreover, the initial selection of weight vector $\hat{\theta}_{0}$ is also very important for SPSA for which random initialization is always possible. If there is any prior information about the system, it is meaningful to select an initial vector accordingly to obtain more precise controls. While using Equation (5.6), we also create a nominal state at every time step, providing a measure of how the estimation procedure is performing as it relies on the updated weight estimate in the neural network. The nominal state creation, 
as defined by Spall (1998), is desirable especially at the very early iterations to monitor the performance of the SPSA algorithm where the perturbations are large. When perturbations become smaller in the later iterations, producing a nominal state may not be necessary. Generating a nominal state follows three basic operational steps:

- Generate the output inventory level value $y_{k+1}^{(+)}$and the value of the objective function $\widehat{L}_{k}^{(+)}$based on $\widehat{\theta}_{k-1}+c_{k} \Delta_{k}, y_{k}$, and $t_{k+1}$.

- Given the system at $y_{k+1}^{(+)}$, generate the output level $y_{k+1}^{(-)}$and the objective function $\widehat{L}_{k}^{(-)}$based on $\hat{\theta}_{k-1}-c_{k} \Delta_{k}, y_{k+1}^{(+)}$, and $t_{k+1}$.

- Use Equation (5.6) to generate $\hat{\theta}_{k}$, and $y_{k+1}$ based on $\hat{\theta}_{k}, y_{k+1}^{(-)}$, and $t_{k+1}$.

The performance of the proposed methodology is highly dependent on the specific properties of our problem such as the substantial amount of noise in the observations, which is highly possible for emergency relief operations. Therefore, we introduce noise into the system and evaluate the performance of the neural network approximation with the Levenberg-Marquardt (LM) algorithm and SPSA algorithms while searching for the optimum gradient descent. The LM algorithm is a good choice for comparison with SPSA, as it appears to be the fastest method (up to ten to one hundred times faster than the standard back-propagation algorithms) for training moderate-sized feed forward neural networks. SPSA, on the other hand, has been successfully implemented for optimization in transportation network analysis problems (Ozguven and Ozbay, 2008). In the following subsections, we will show the comparative results of the proposed online control methodology based on these algorithms, namely the neural network approximation with Levenberg-Marquardt (LM) algorithm and SPSA algorithm. 
For the model-free feedback control methodologies studied, the inputs and outputs shown in Table 5.1 are present.

Table 5.1 Inputs and Outputs for the Model-free Feedback Control Methodology

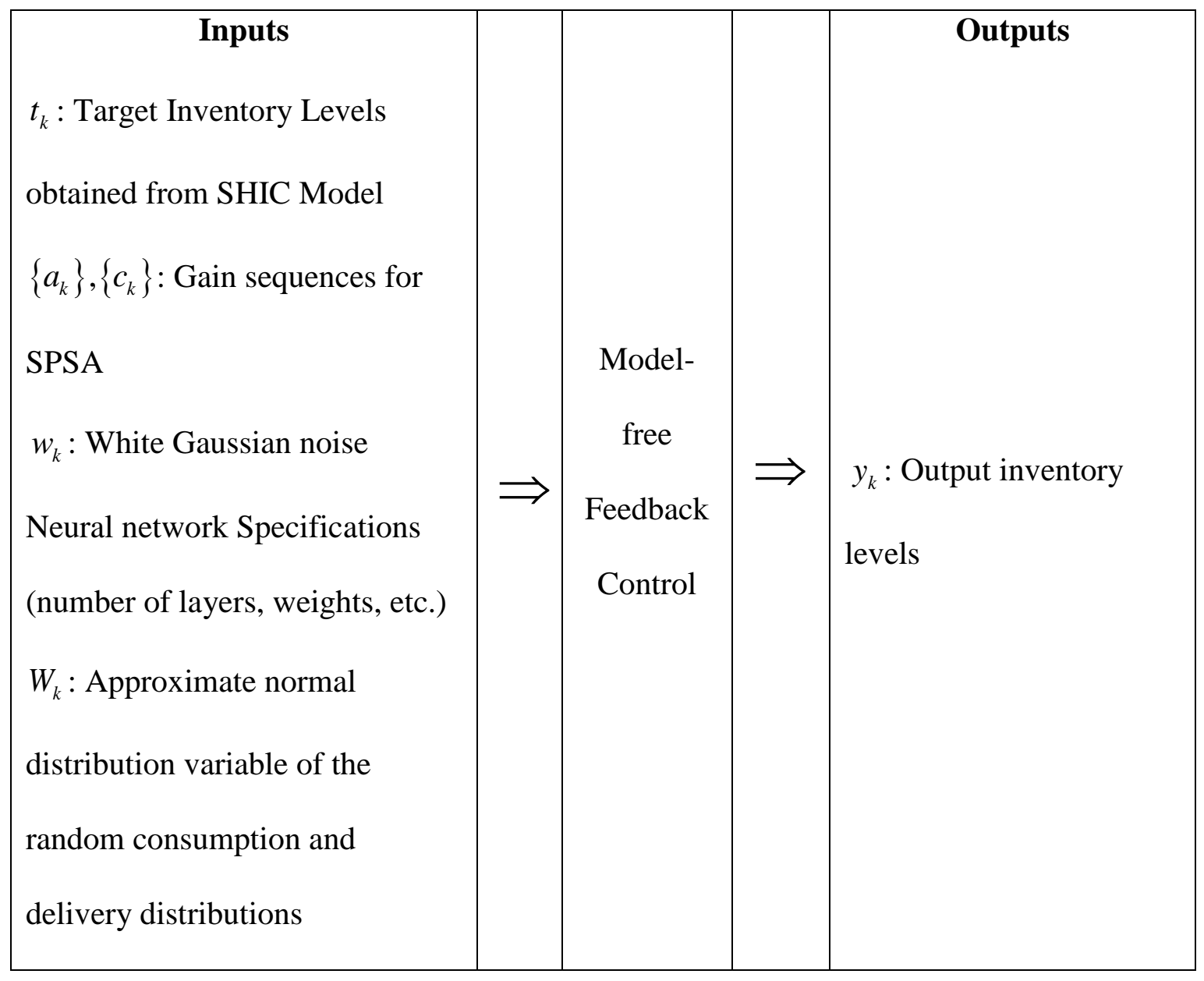

\subsubsection{Numerical Study: Target Levels Kept Constant}

In our tests, we consider a feed-forward neural network as the functional approximator, with five inputs, two outputs, and two hidden layers with 10 neurons in each layer. This gives $50+100+20+22=192$ weights (including bias) to be estimated throughout the procedure. This neural network, of course, has no knowledge of disaster relief system dynamics and the SHIC model. The hidden layer nodes are tangent sigmoid 
functions (i.e., $\frac{1}{1+e^{-z}}$ for an input $z$ ), and the output node has a linear transfer function. Only the most recent measurement and the next target are given into the neural network. Moreover, the size of the training set for the neural network is determined based on the number of hidden neurons (Ripley, 1996). According to the criteria given in Ripley (1996), the training set typically requires 10 to 100 times the number of neurons within the hidden layers. Therefore, given the number of total neurons as 20 in the two hidden layers, we select 1,000 as the size of our training set. After the training, LM and SPSA algorithms are used to minimize the unconstrained minimization problem given in Equation (5.4), and a comparative analysis is presented at the end.

The initial point vector, $\hat{\theta}_{0}$, is taken as an independent uniformly distributed vector between $[-1,1]$ for both algorithms, and the SPSA perturbation vector $\left(\Delta_{k}\right)$ choices are varied randomly and symmetrically around zero. The choice of gain sequences has utmost importance for the adequate performance of the algorithm, and they are selected as $a_{k}=a=0.001$ and $c_{k}=c=0.005$ at the start and are gradually increased until reaching a relatively sufficient convergence rate (after several tuning iterations to maximize the rate of the decrease in the total system rms error). The values satisfy $0.001<a<0.5$ and $0.005<c<0.1$. The objective function is based on $A_{k}=A$, a constant diagonal weighting matrix, with a value of 0.5 for the diagonal elements.

The parameter values to create the consumption and delivery data and to train the neural network are taken from the example numerical study for the base two-commodity analysis of the SHIC model in Chapter 4. Suppose we start the analysis with the demand 
scenario 5 , where $C_{k}=[38,36], D_{k}=[18,16]$. The multi-commodity SHIC model gives a target vector as $t_{k}=[26,27]$. We generate the data using random consumption and deliveries within the range of the initial values of consumption and deliveries and add noise to the data. This is done for the sake of illustration to see how the neural network will work (in terms of both computational times and rms errors) while searching for the target inventory levels with these random characteristics and the non-additive noise in the system. Moreover, the performance of the algorithms will be evaluated by the root mean square error (RMSE) values for the observations. After the function approximator reaches a local or global optimum for the first target value $t_{k}=[26,27]$, we change the consumption and delivery vectors to $C_{k}=[48,56], D_{k}=[28,36]$ to make it possible to observe the performance of the algorithms. With these values, given that other parameters are kept constant, we obtain the target vector $t_{k}=[33,42]$ according to demand 10 . Now, the algorithms are again looking for the best controlling strategy to minimize the objective function and to obtain the possible RMSE values for the data generated by the MC-SHIC model within the range of the initial values of consumption and deliveries. For each studied case, number of runs, number of iterations in each run, RMSE errors, objective function and decision parameter values, and computational times (wall-clock times) are reported. All the table values calculated are based on the sample mean of ten independent runs. The computational time of the MATLAB implementations on a 3.16 $\mathrm{GHz}$ Intel Xeon (R) PC is presented for each case.

Introducing the system with the stochastic consumptions, deliveries and random noise given as in Equation (5.6), the results for the case with demand $5\left(t_{k}=[26,27]\right)$ are 
given in Table 5.2 for each group of runs. In this case, the LM algorithm performs better in terms of computational time, considering the relatively shorter time it takes to find an optimal solution. However, the SPSA algorithm works more efficiently in reaching the solution given the lower RMSE errors. Note that reaching optimal inventory control levels is one of the most important concerns for online emergency disaster management operations.

Table 5.2 Performance of the Levenberg-Marquardt and SPSA Algorithms for Target Demand 5

\begin{tabular}{|c|c|c|c|c|c|c|c|c|c|c|c|}
\hline \multicolumn{12}{|c|}{$\begin{array}{c}\text { Target Demand }=\left[\begin{array}{ll}26 & 27\end{array}\right] \\
\left(\text { Total number of maximum allowed evaluations }\left(N_{\max }\right)=1000\right)\end{array}$} \\
\hline \multicolumn{6}{|c|}{$\mathbf{L M}$} & \multicolumn{6}{|c|}{ SPSA } \\
\hline Run & $\mathbf{N}$ & RMSE & $\mathbf{y}_{1}$ & $\mathbf{y}_{2}$ & $\begin{array}{l}\text { Time } \\
(\text { sec) }\end{array}$ & Run & $\mathbf{N}$ & RMSE & $\mathbf{y}_{1}$ & $\mathbf{y}_{2}$ & $\begin{array}{l}\text { Time } \\
(\text { sec) }\end{array}$ \\
\hline 1 & 172 & 0 & 26.0 & 27.0 & 1.91 & 1 & 66 & 0.354 & 26.4 & 27.3 & 15.55 \\
\hline 2 & 446 & 0 & 26.0 & 27.0 & 4.47 & 2 & 78 & 0.381 & 26.5 & 26.8 & 20.66 \\
\hline 3 & 5 & 3.54 & 30.0 & 30.0 & 0.44 & 3 & 92 & 1.082 & 27.5 & 27.3 & 29.15 \\
\hline 4 & 7 & 3.54 & 30.0 & 30.0 & 0.41 & 4 & 60 & 0.453 & 25.6 & 26.5 & 14.53 \\
\hline 5 & 14 & 0.95 & 27.2 & 27.6 & 0.46 & 5 & 81 & 1.200 & 27.2 & 28.2 & 24.66 \\
\hline 6 & 322 & 0 & 26.0 & 27.0 & 3.66 & 6 & 55 & 0.224 & 26.1 & 27.3 & 12.05 \\
\hline 7 & 65 & 0 & 26.0 & 27.0 & 0.62 & 7 & 41 & 0.354 & 25.5 & 27.0 & 4.09 \\
\hline 8 & 265 & 0 & 26.0 & 27.0 & 2.92 & 8 & 35 & 0.224 & 25.7 & 27.1 & 4.82 \\
\hline 9 & 106 & 3.54 & 30.0 & 30.0 & 1.42 & 9 & 98 & 0.212 & 26.0 & 27.3 & 30.11 \\
\hline 10 & 44 & 0.32 & 26.4 & 27.2 & 0.92 & 10 & 85 & 0.430 & 26.6 & 27.1 & 26.26 \\
\hline \multicolumn{6}{|c|}{ Averages } & \multicolumn{6}{|c|}{ Averages } \\
\hline & 145 & 1.19 & 27.4 & 28.0 & 1.72 & & 69 & 0.491 & 26.3 & 27.2 & 18.19 \\
\hline
\end{tabular}

In the next scenario (Table 5.3), the LM with the trained neural network and SPSA algorithms are run by changing the consumption and delivery amounts to reach the new target inventory levels, namely an increase to demand $10\left(t_{k}=[33,42]\right)$. This may always occur in the aftermath of disasters due to the increased vital supply consumption 
in the emergency shelters. The performance of the LM algorithm indicates that less than half of the ten runs reach the optimal solutions, whereas SPSA gives better RMSE, objective function, and inventory level values.

Table 5.3 Performance of the Levenberg-Marquardt and SPSA Algorithms for Target Demand 10

\begin{tabular}{|c|c|c|c|c|c|c|c|c|c|c|c|}
\hline \multicolumn{12}{|c|}{$\begin{array}{c}\text { Target Demand }=\left[\begin{array}{ll}33 & 42\end{array}\right] \\
\left(\text { Total number of maximum allowed evaluations }\left(\mathrm{N}_{\max }\right)=1000\right)\end{array}$} \\
\hline \multicolumn{6}{|c|}{ LM } & \multicolumn{6}{|c|}{ SPSA } \\
\hline Run & $\mathbf{N}$ & RMSE & $\mathbf{y}_{1}$ & $\mathbf{y}_{2}$ & $\begin{array}{l}\text { Time } \\
\text { (sec) }\end{array}$ & Run & $\mathbf{N}$ & RMSE & $\mathbf{y}_{1}$ & $\mathbf{y}_{2}$ & $\begin{array}{l}\text { Time } \\
\text { (sec) }\end{array}$ \\
\hline 1 & 4 & 4.50 & 37.5 & 37.5 & 0.56 & 1 & 7 & 0.791 & 34.1 & 42.2 & 2.86 \\
\hline 2 & 31 & 0 & 33.0 & 42.0 & 0.69 & 2 & 41 & 0.608 & 32.5 & 41.3 & 17.42 \\
\hline 3 & 309 & 0 & 33.0 & 42.0 & 2.98 & 3 & 60 & 0.852 & 32.2 & 41.1 & 21.66 \\
\hline 4 & 216 & 4.50 & 37.5 & 37.5 & 2.28 & 4 & 102 & 0.224 & 33.3 & 41.9 & 36.19 \\
\hline 5 & 1000 & 4.50 & 37.5 & 37.5 & 13.25 & 5 & 76 & 0.500 & 32.3 & 41.9 & 26.62 \\
\hline 6 & 36 & 0 & 33.0 & 42.0 & 0.72 & 6 & 58 & 1.217 & 31.6 & 41.0 & 19.85 \\
\hline 7 & 24 & 4.50 & 37.5 & 37.5 & 0.61 & 7 & 66 & 1.020 & 31.8 & 41.2 & 20.86 \\
\hline 8 & 18 & 1.20 & 34.2 & 40.8 & 0.52 & 8 & 36 & 0.854 & 33.5 & 40.9 & 14.42 \\
\hline 9 & 640 & 0 & 33.0 & 42.0 & 7.05 & 9 & 18 & 0.583 & 32.2 & 42.2 & 9.02 \\
\hline 10 & 96 & 4.50 & 37.5 & 37.5 & 1.46 & 10 & 72 & 0.828 & 32.6 & 40.9 & 30.11 \\
\hline \multicolumn{6}{|c|}{ Averages } & \multicolumn{6}{|c|}{ Averages } \\
\hline & 237 & 2.37 & 35.4 & 39.6 & 3.01 & & 54 & 0.748 & 32.6 & 41.4 & 19.90 \\
\hline
\end{tabular}

\subsubsection{Numerical Study: Fluctuating Target Levels Due to Stochasticities}

In this subsection, different from the previous analyses, we let the target inventory levels change at every time step in the multi-commodity SHIC model. This case represents a worst-case scenario with the highest stochasticity on the inventory levels. This, indeed, makes it harder for the control system to calculate the gradient descent direction. As observed from Table 5.4, although the performances of both algorithms decrease, LM significantly moves away from the vicinity of the target values, leading to 
higher RMSE errors, and the performance of LM is not very satisfactory. Only three out of ten runs give an acceptable RMSE error value. SPSA, on the other hand, gives better RMSE values, although the computational time values are still significantly larger than LM. This indicates that SPSA works more efficiently in calculating safety stock values when the variability of target inventory levels changes at every time step.

Table 5.4 Performance of the Levenberg-Marquardt and SPSA Algorithms with the Variable Target Vector

\begin{tabular}{|c|c|c|c|c|c|c|c|}
\hline \multicolumn{8}{|c|}{$\begin{array}{l}\text { Variable Target Demand } \\
\left(\text { Total number of maximum allowed evaluations }\left(N_{\max }\right)=1000\right)\end{array}$} \\
\hline \multicolumn{4}{|c|}{$\mathbf{L M}$} & \multicolumn{4}{|c|}{ SPSA } \\
\hline Run & $\mathbf{N}$ & RMSE & $\begin{array}{l}\text { Time } \\
(\text { sec) }\end{array}$ & Run & $\mathbf{N}$ & RMSE & $\begin{array}{l}\text { Time } \\
\text { (sec) }\end{array}$ \\
\hline 1 & 486 & 4.56 & 8.92 & 1 & 36 & 0.523 & 19.62 \\
\hline 2 & 15 & 2.55 & 2.51 & 2 & 82 & 1.120 & 56.01 \\
\hline 3 & 1000 & 10.88 & 23.63 & 3 & 102 & 0.593 & 79.69 \\
\hline 4 & 765 & 8.65 & 13.55 & 4 & 76 & 0.987 & 28.22 \\
\hline 5 & 8 & 0 & 1.46 & 5 & 44 & 0.752 & 17.03 \\
\hline 6 & 980 & 12.65 & 20.32 & 6 & 48 & 0.683 & 10.22 \\
\hline 7 & 36 & 0 & 2.92 & 7 & 56 & 1.227 & 48.85 \\
\hline 8 & 1000 & 9.69 & 25.25 & 8 & 99 & 0.286 & 66.65 \\
\hline 9 & 19 & 3.03 & 3.66 & 9 & 69 & 0.860 & 25.76 \\
\hline 10 & 345 & 0 & 6.21 & 10 & 50 & 0.792 & 29.05 \\
\hline \multicolumn{4}{|c|}{ Averages } & \multicolumn{4}{|c|}{ Averages } \\
\hline & 465 & 5.20 & 10.84 & & 66 & 0.782 & 38.11 \\
\hline
\end{tabular}

\subsubsection{Discussion}

The multi-commodity SHIC model is used to determine optimal target inventory levels mainly for offline inventory management purposes and to generate simulated data related to the stochastic consumption and deliveries of vital commodities needed for the FA (neural network) in the context of our online control framework. With the target 
values and simulated data obtained using the multi-commodity SHIC model, the neural network approximation with the LM algorithm and the SPSA algorithm are used to solve the unconstrained nonlinear optimization problem shown in Equation (5.4) to account for the deviation between the actual inventory levels and the target ones. The need to use a neural network-based functional approximator for the online theoretical control disaster framework comes from the fact that the underlying dynamic system equations are not known to derive the control laws.

The analysis and comparison of LM and SPSA algorithms provide useful insights into the development of solution techniques for emergency relief systems that can be highly stochastic. The performance comparison of these two algorithms is based on the analysis of the objective functions, the optimal inventory levels, and the overall computational times. Number of iterations is not used for comparison, as the computation time of a single iteration for each algorithm is different. The results of the functional approximation-based online management numerical study suggest that the performance of the SPSA significantly improves the optimality of the solution as the amount of system stochasticity is increased. Note that high levels of stochasticities in the flow of emergency supplies are most likely to be encountered in the aftermath of disasters due to fluctuations in consumption and available resources, and due to disruptions in the transportation network.

The analysis results indicate that there is a substantial amount of difference between the algorithms in terms of computational times where neural network approximation with the LM algorithm performs better than SPSA. One of the important factors causing that difference is the creation of the nominal state for SPSA while 
calculating the weight vector fed into the neural network at every step. This nominal state enables us to monitor the performance of the SPSA algorithm so that we can change the gain sequences accordingly to achieve better results. It is actually one of the reasons SPSA is doing a better job than the LM algorithm in obtaining inventory levels closer to target values.

Finally, it is natural to ask whether the aforementioned stochastic approximation with SPSA is converging to the target inventory stock values obtained via the multicommodity SHIC model. To observe the behavior of the SPSA algorithm, an additional analysis is conducted, where the initial point, the gain sequences, the perturbation vector, and the random noise are selected accordingly to make averaging possible. Figure 5.3 shows the results after averaging 100 system runs to reach the target inventory level $t_{k}=[33,42]$ obtained via the multi-commodity SHIC model. In fact, the resulting final RMSE errors and optimal inventory level values are worse than the ones seen in Table 5.3. This is due to the aforementioned parameter selection to make it possible for averaging with similar runs and therefore to see the convergence behavior clearly. From Figure 5.3, it can be stated that the SPSA algorithm exhibits good performance in terms of converging to the target inventory level values. There are several jumps throughout the analysis, mainly due to the randomness coming from the stochastic consumptions and deliveries; however, the algorithm reaches a steady state after approximately 125 iterations. 


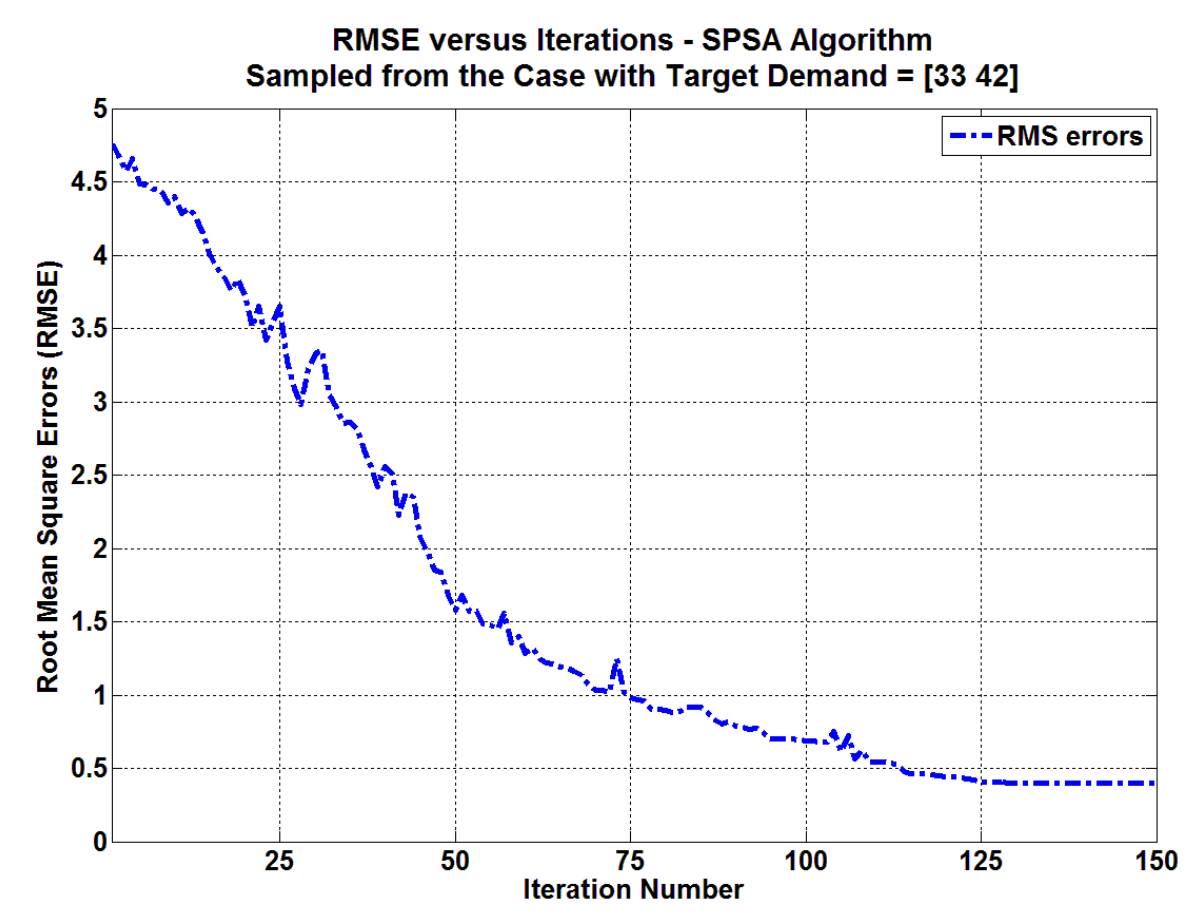

Figure 5.3 RMSE versus Iterations (SPSA Algorithm)

To sum up, neural network-based approach shows better performance in terms of computational time. However, the SPSA algorithm clearly reaches to near-optimal inventory levels closer to the target ones obtained from the planning model SHIC. Therefore, planner or decision maker should be careful to choose one of these two algorithms based on the specific disaster conditions on hand. Intuitively, if time is a major concern as in the case of sudden onset disasters, it may be logical to use the neural networks based approach. On the other hand, for slow onset disasters, where calculation of optimal inventory levels more accurately may be more important than time, SPSA can be efficiently used to obtain the emergency inventory levels. 


\subsection{Model 2: Continuous Time Model Predictive Control Approach}

In the aftermath of disasters, it is very difficult, and almost impossible, to maintain optimal inventory levels due to possible disruptions in the transportation and infrastructure system, and due to random and highly dynamic changes in evacuee demand and available resources. Therefore, there is definitely a need for a robust emergency management system, where offline planning and production policies can be integrated with an online inventory management strategy to keep track of demand fluctuations for vital supplies (food, water, medical supplies, etc.) as well as transportation network disruptions during the disaster relief period. This integrated real-time inventory management system should not only allow the efficient usage and distribution of emergency supplies, but also offer the ability to be integrated with emerging ITS technologies such as Radio Frequency Identification Devices (RFID) for online commodity tracking and logistics. To make such integration possible, in the previous section, we proposed a neural network-based functional approximator and SPSA algorithm. However, these methods have crucial disadvantages, stated as follows:

- Models do not provide a means of analytically computing the real-time trajectory of the flow of vital supplies and emergency inventory levels.

- Convergence to optimal inventory values is not necessarily satisfied and directly depends on the selection and performance of the system parameters. 
- This dependence on the parameters creates the problem of instability for the emergency relief system, which should be avoided.

- Constraints in terms of inventory capacity and limitations for the delivery of supplies that are possibly encountered in real-world disaster systems cannot be handled with these methods.

Given these limitations of the neural network and SPSA-based models, there is apparently a need for a better methodology to represent emergency inventory operations in the aftermath of a disaster, which should effectively address needs based on online tracking, mathematical convergence, stability, and real-life constraints. Such a model can be obtained by employing exponential functions to control the flow of vital supplies that ensure exponential decay during the convergence process to optimal inventory values.

In this section, to address the aforementioned limitations, we propose a comprehensive closed-loop humanitarian emergency management framework that monitors inventory in real time (it is possible to use RFID tracking technology for this purpose) with the objective of feeding this inventory information back into a novel multicommodity stochastic humanitarian inventory management model developed by Ozguven and Ozbay (2011). The aim is to use a model predictive control approach (MPC) that has the ability to compute the trajectory of the commodity inventory level changes to optimize the future behavior of the disaster inventory management framework. Moreover, another purpose is to obtain a continuous time model predictive control rather than a discrete version. As the continuous approach requires a convolution-based calculation for inventory control rather than a direct iteration of the discrete case, it becomes technically more complicated to design a continuous time MPC. For this purpose, we use the 
approach first developed by Wang (2001), where the idea is to model the control trajectory based on pre-chosen orthonormal basis functions. The core of this methodology is to determine the future control trajectory of the inventory levels that converges to a constant for the chosen control horizon. Within this approach, the derivative of the control also converges to zero exponentially. This model has three basic advantages:

- The methodology is flexible and stable to use a multi-variable state space model.

- The future trajectory is predicted using analytical techniques.

- The model allows solving the problem including system constraints with an exponential rate of convergence.

The continuous time model predictive control methodology works based on a receding horizon control approach on a moving horizon window, with a length $T_{p}$ (as a forecast of the planned emergency relief operations) for our future inventory trajectories. The moving horizon window simply represents the time-dependent window for which we obtain our future emergency commodity inventory levels. The receding horizon control method uses only the first sample of the control signal to calculate the actual control input while neglecting the rest of the moving horizon trajectory within the moving horizon window. The idea of receding horizon control can be seen in Figure 5.4: 


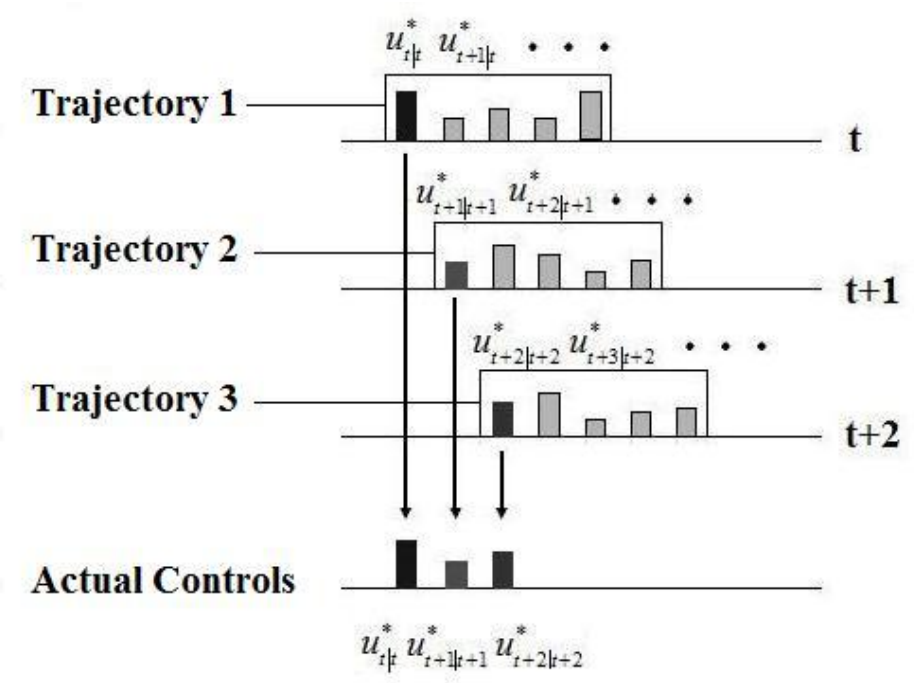

Figure 5.4 Concept of Receding Horizon (Adapted for the Online Disaster Management Problem from Kwon and Han, 2005)

Receding horizon control (RHC) has several advantages over finite horizon control models. First of all, fixed horizon optimization leads to a control sequence that begins at the current time and ends at some future time. This fixed horizon solution suffers from two potential drawbacks:

- Something unexpected may happen to the system at some time over the future interval that was not predicted by (or included in) the model. This would render the fixed control choices obsolete.

- As one approaches the final time, the control law typically "gives up trying", as there is too little time to achieve anything useful in terms of objective function reduction.

RHC addresses the above two problems by solving the problem for optimality with current and future constraints for the initial conditions but applying only the first step of the trajectory in the resulting optimal control strategy. This approach is repeated for the next state until the end. Therefore, the idea is to obtain inventory levels of 
emergency supplies for a predicted horizon given the information at the current time to predict the future, and a cost function to be minimized related to an error function based on the difference between the desired and the actual responses.

In the following subsections, we will introduce the overall methodology for the aforementioned continuous time model predictive control (CMPC) approach and the integration of the CMPC technique and the multi-commodity SHIC model.

\subsubsection{Motivation and Intuitive Explanation}

As mentioned in the previous sections, online control of inventories and flow of supplies is necessary to cope with the stochasticity involved in the aftermath of a disaster. Intuitively, this noise depends on the disruptions due to the expectations of fluctuation in demand for vital supplies, damaged transportation networks, lost inventories, etc. In the model, disruptions in the disaster conditions (noise) are determined in terms of the coefficient of variation in the model, which is the ratio of the standard deviation to the mean.

With the CMPC model, we can also model the constraints that are present in the real-life disaster operations. The first type of constraint represents the limitations on the inventory levels that can be stocked. An intuitive explanation for this constraint would be the unavailability of warehouses, or partial damage of the inventory depots. For example, after the cyclone in Mozambique in 2007, delivery of relief supplies to temporary camps for people affected by the disaster was not easy in the face of severe inventory capacity constraints (unavailability of locations to store commodities) (Amin et al., 2008). To solve the problem, the National Emergency Operations Center maintained a registry of 
the temporary camps, and the delivery of daily supplies for these camps was coordinated very slowly.

The second type of constraints that can be modeled within the CMPC framework include the limitations for flow of supplies, which represents the constraints on the delivery of relief supplies to the emergency shelters. In the aftermath of a disaster, deliveries to the shelters may be limited due to a damaged roadway network, or unavailable emergency supply vehicles. These constraints were all present during the recent disasters such as Hurricane Katrina or the earthquakes in Haiti and Japan. Depending on political problems, there are even more uncommon constraints on the flow of supplies such as North Korea's block of relief shipments from South Korea during the response to the April 2004 train explosion.

The third type of constraint is not as intuitive as the other constraints. These are basically limitations on the rate of change in the flow of supplies in the emergency management framework. The disruptions and the uncertainties within the emergency relief system can cause sudden rate changes in the supply flow and that rate change will gradually affects delivery operations. Here, a positive rate will mean that the flow of supplies will increase in future time steps whereas a negative rate will lead to a decrease in the deliveries gradually. By controlling this rate, the planner or emergency official can make decisions on increasing and decreasing the deliveries to a shelter where the number of people located in that shelter change rapidly. This will provide a controlling mechanism during the disaster relief operations.

With this constrained model that can capture real-life cases, we focus on the target inventory levels calculated via the planning model, SHIC, and minimize the cost 
associated with the error between those target levels and the current output inventory levels obtained from the CMPC model. This cost can be intuitively considered as the cost of not having enough inventory stock for vital supplies during the disaster relief operations.

Moreover, as clearly stated by Whybark (2007), the management of the physical commodities in storage can be improved if there were more effective tracking and monitoring systems. Using the aforementioned objectives and constraints, CMPC model obtains the future trajectory of emergency inventory levels that can be efficiently used by planners and decision makers for real-time commodity tracking.

\subsubsection{Methodology}

\subsubsection{Basic Assumptions}

To be able to use the CPMC technique, we make the following assumptions on representing real-life conditions:

- The inventory system is assumed to be a multi-commodity framework that operates within a moving horizon window.

- Demand and delivery distributions are assumed to be known and obtained from the SHIC model. An RFID implementation will provide the necessary data to simulate accurate delivery and consumption distributions. In the absence of such data, the SHIC model is used as the base data simulator.

- Inventory is assumed to be continuously reviewed and proper inventory control revisions are made by the planner at predetermined time steps. If there are no emergency officials present to review the current inventory levels and flow of 
supplies, the model will continuously predict the future inventory levels using the last available information in the last time step known (last review).

- Costs in the objective function are assumed to be based on the error between the target inventory levels obtained via the SHIC model and the output inventory levels obtained from the CMPC technique.

- White Gaussian noise is assumed to represent the stochastic conditions in the aftermath of a disaster and is used to better simulate the extreme complexity expected under real-world emergency conditions.

\subsubsection{Formulation}

First, we will introduce the inventory control model structure in terms of a statespace model for our multi-variable ( $m$ inputs and $k$ outputs, where the number of outputs is always less than or equal to the number of inputs) disaster inventory management framework. Note that the variables with a subscript $m$ represent the original state-space formulation, whereas the variables without the subscript represent the augmented state-space formulation. Our continuous time-invariant model is represented as follows:

$$
\begin{gathered}
\dot{x}_{m}(t)=A_{m} x_{m}(t)+B_{m} u(t) \\
y(t)=C_{m} x_{m}(t)+D_{m} u(t)
\end{gathered}
$$

Here, $x_{m}(t)$ represents the inventory state vector of dimension $l$, whereas $u(t)$ is the input (manipulated) signal vector (representing the flow of emergency supplies for inventories) of dimension $m$ and $y(t)$ is the output inventory level vector (inventory stock values to be optimized) of dimension $k$. In the formulation, $A_{m}, B_{m}, C_{m}$, and $D_{m}$ 
are state-space matrices with the dimensions $l \times l, l \times m, k \times l$, and $k \times m$ respectively. Note that a linear time-invariant system is a system described by linear differential equations with constant coefficients. Thus, the system is time invariant if the parameters do not change as a function of time.

Given this model, the principle of receding horizon control states that only current information of the emergency inventory system is used to obtain the strategy for controlling the flow of vital supplies, and as a result, to calculate the predicted inventory outputs. Therefore, due to the nature of the receding horizon control, we assume that the control $u(t)$ cannot affect $y(t)$ at the same time. With this statement, $D_{m}$ equals 0 in our model that becomes:

$$
\begin{gathered}
\dot{x}_{m}(t)=A_{m} x_{m}(t)+B_{m} u(t) \\
y(t)=C_{m} x_{m}(t)
\end{gathered}
$$

The next step is to obtain an augmented space for our state-space model in Equation (5.9). To obtain this, we define the following state variable:

$$
x(t)=\left[\begin{array}{c}
\dot{x}_{m}(t) \\
y(t)
\end{array}\right] .
$$

Then, using this new state variable, we obtain the augmented space state formulation using Equation (5.9) and taking the derivative of $\dot{x}_{m}(t)$ as:

$$
\begin{gathered}
\dot{x}(t)=\left[\begin{array}{c}
\ddot{x}_{m}(t) \\
\dot{y}(t)
\end{array}\right]=\left[\begin{array}{c}
A_{m} \dot{x}_{m}(t)+B_{m} \dot{u}(t) \\
C_{m} \dot{x}_{m}(t)
\end{array}\right]=A\left[\begin{array}{c}
\dot{x}_{m}(t) \\
y(t)
\end{array}\right]+B \dot{u}(t)=A x(t)+B \dot{u}(t) \\
y(t)=C x(t)=C\left[\begin{array}{c}
\dot{x}_{m}(t) \\
y(t)
\end{array}\right]
\end{gathered}
$$

where the augmented space matrices can be obtained as follows ( $I$ : identity matrix, 0 : zero matrix): 


$$
\begin{gathered}
A=\left[\begin{array}{cc}
A_{m} & 0_{m}^{T} \\
C_{m} & 0_{k \times k}
\end{array}\right] \\
B=\left[\begin{array}{c}
B_{m} \\
0_{k \times m}
\end{array}\right] \\
C=\left[\begin{array}{ll}
0_{m} & I_{k \times k}
\end{array}\right]
\end{gathered}
$$

The significant point about this augmented model is that it should not be uncontrollable and unobservable given the unstable and stochastic conditions of our emergency relief framework. These controllability and observability properties will lead to achieving a desired closed-loop control performance and a successful observer for the continuous time predictive control model. To investigate the controllability and observability features, we focus on the transfer function $G_{m}(s)$ for the augmented statespace model in Equation (5.9) and $G(s)$ for the original state space formulation. The transfer function of our continuous time-invariant state-space model is given as below:

$$
G_{m}(s)=C_{m}\left(s I-A_{m}\right)^{-1} B_{m}
$$

The space state matrix system $\left(A_{m}, B_{m}, C_{m}\right)$ is called a realization of $G_{m}(s)$ when this equation holds. This realization set $\left(A_{m}, B_{m}, C_{m}\right)$ is called minimal if no other realizations of $G_{m}(s)$ have a smaller dimension. The distinctive feature of the minimal realization is that a minimal realization is both controllable and observable (Kalman, 1963).

With the minimal realization of the augmented system $(A, B, C)$, the transfer function of the augmented state-space model in Equation (5.12) has the following representation:

$$
G(s)=C(s I-A)^{-1} B
$$


The relationship between the two transfer functions $G_{m}(s)$ and $G(s)$ has utmost importance for our model to be applicable to our emergency management framework. First, let's focus on our lower triangular matrix $(s I-A)$ :

$$
(s I-A)=s\left[\begin{array}{cc}
I_{m} & 0_{m}^{T} \\
0_{m} & I_{k x k}
\end{array}\right]-\left[\begin{array}{cc}
A_{m} & 0_{m}^{T} \\
C_{m} & 0_{k x k}
\end{array}\right]=\left[\begin{array}{cc}
s I_{m}-A_{m} & 0_{m}^{T} \\
-C_{m} & s I_{k x k}
\end{array}\right]
$$

We can obtain the inverse of this triangular matrix as:

$$
(s I-A)^{-1}==\left[\begin{array}{cc}
\left(s I_{m}-A_{m}\right)^{-1} & 0_{m}^{T} \\
s^{-1} C_{m}\left(s I_{m}-A_{m}\right)^{-1} & s^{-1} I_{k x k}
\end{array}\right]
$$

Using this equality and re-writing matrices $B$ and $C$, we can write the following transfer function for the augmented space in Equation (5.13):

$$
\begin{gathered}
G(s)=C\left[\begin{array}{cc}
\left(s I_{m}-A_{m}\right)^{-1} & 0_{m}^{T} \\
s^{-1} C_{m}\left(S I_{m}-A_{m}\right)^{-1} & s^{-1} I_{k x k}
\end{array}\right] B=\left[0_{m} I_{k x k}\right]\left[\begin{array}{cc}
\left(s I_{m}-A_{m}\right)^{-1} & 0_{m}^{T} \\
s^{-1} C_{m}\left(S I_{m}-A_{m}\right)^{-1} & s^{-1} I_{k x k}
\end{array}\right]\left[\begin{array}{c}
B_{m} \\
0_{k x m}
\end{array}\right] \\
=s^{-1} C_{m}\left(S I_{m}-A_{m}\right)^{-1} B_{m}=\frac{1}{s} G_{m}(s)
\end{gathered}
$$

This realization is both controllable and observable if and only if $G_{m}(s)$ has no zero at $s=0$. The zeros of a multi-input multi-output transfer function are those values of $s$ that make the matrix $G_{m}(s)$ lose rank. As stated by Barry et al. (2004), the loss of linearly independency leads to an output inventory value of zero, which is not desired. Hence, with the assumption that the disaster system model has no zero at $s=0$ and has a minimal realization, the transfer function of the augmented model, $G(s)$, has a minimal structure and is both controllable and observable. 


\subsubsection{Solution Approach}

The difficulty in solving the system in Equation (5.11) comes from the fact that we do not have closed form equations representing the disaster relief operations. Our planning model SHIC represents the real-life conditions for the CMPC model. Given this information, one of the most important challenges is to be able to optimally control the future disaster inventory trajectory. Therefore, we focus on a time variable $\tau$ within the moving horizon window $\left[t_{i}, t_{i}+T_{p}\right]$ and calculate the derivative of the control, $\dot{u}(\tau)$, for $0 \leq \tau \leq T_{p}$ as follows:

$$
\dot{u}(\tau)=-K_{m p c} x(\tau)
$$

where $K_{m p c}$ is the state gain matrix. Here, our closed-loop system can be described by the following equation:

$$
x(\tau)=e^{\left(A-B K_{m p c}\right) \tau} x\left(t_{i}\right)
$$

First of all, to be able to model the trajectory that represents the change in the control $u(\tau)$, namely, the change in the flow of vital supplies in Equation (5.17), we will use the orthonormal functions (Wang, 2001).

A sequence of real functions $l_{i}(t), i=1,2, \ldots$ is said to form an orthonormal set over the interval $[0, \infty)$ if they have the following properties:

$$
\begin{gathered}
\int_{0}^{\infty} l_{i}^{2}(\tau) d \tau=1 \\
\int_{0}^{\infty} l_{i}(\tau) l_{j}(\tau) d \tau=0 \quad i \neq j
\end{gathered}
$$


Another important property that we will use is that a set of orthonormal functions $l_{i}(\tau)$ is said to be complete if the following relation holds:

$$
\int_{0}^{\infty} f(\tau) l_{i}(\tau) d \tau=0
$$

for only the values of $i$ if the squared $f(\tau)$ satisfies

$$
\int_{0}^{\infty} f(\tau)^{2} d \tau=0
$$

Laguerre functions (Lee, 1960) are orthonormal functions that do satisfy the orthonormal and complete properties aforementioned. The set of Laguerre functions is defined as follows (Lee, 1960):

$$
\begin{gathered}
l_{1}(\tau)=(\sqrt{2 p}) e^{-p \tau} \\
l_{2}(\tau)=(\sqrt{2 p})(-2 p \tau+1) e^{-p \tau} \\
\vdots \\
l_{i}(\tau)=(\sqrt{2 p}) \frac{e^{p \tau}}{(i-1) !} \frac{d(i-1)}{d \tau(i-1)}\left[\tau^{i-1} e^{-2 p \tau}\right]
\end{gathered}
$$

Here, $p>0$ is a time scaling factor for the Laguerre functions that determines the exponential decay rate. The interesting point about the Laguerre functions is that they easily calculate Laplace transforms that form the set of Laguerre network as follows, where $L_{i}(s), i=1,2, \ldots$ are the Laguerre filters:

$$
\begin{gathered}
L_{1}(s)=\int_{0}^{\infty} l_{1}(\tau) e^{-s \tau} d \tau=\frac{(\sqrt{2 p})}{(s+p)} \\
L_{2}(s)=\int_{0}^{\infty} l_{2}(\tau) e^{-s \tau} d \tau=\frac{(\sqrt{2 p})(s-p)}{(s+p)^{2}} \\
\vdots \\
L_{i}(s)=\int_{0}^{\infty} l_{i}(\tau) e^{-s \tau} d \tau=\frac{(\sqrt{2 p})(s-p)^{i-1}}{(s+p)^{i}}
\end{gathered}
$$


As observed, Laguerre filters can be computed analytically in a practical and easy way where all the poles are on the same location $p$ and all the filters other than the first one are first-order filters. These filters can be obtained within our state-space inventory control framework by first defining the state vector $L(\tau)=\left[l_{1}(\tau) l_{2}(\tau) \ldots l_{N}(\tau)\right]^{T}$. Then, with the help of Equation (5.22), the Laguerre system satisfies the following state-space equation, where the initial condition of the state vector is $L(0)=\sqrt{2 p}\left[\begin{array}{ll}1 & 1 \ldots 1\end{array}\right]^{T}$ (Wang and Cluett, 2000):

$$
\left[\begin{array}{c}
i_{1}(\tau) \\
i_{2}(\tau) \\
\vdots \\
i_{N}(\tau)
\end{array}\right]=\left[\begin{array}{cccc}
-p & 0 & \ldots & 0 \\
-2 p & -p & \ldots & 0 \\
\vdots & \ddots & \ddots & \vdots \\
-2 p & \ldots & -2 p & -p
\end{array}\right]\left[\begin{array}{c}
l_{1}(\tau) \\
l_{2}(\tau) \\
\vdots \\
l_{N}(\tau)
\end{array}\right]
$$

Here, $N$ is the number of terms used in the Laguerre system to capture the control signal. Solving this equation gives the set of Laguerre functions as (Wang and Cluett, 2000):

where $A_{p}=\left[\begin{array}{cccc}-p & 0 & \ldots & 0 \\ -2 p & -p & \ldots & 0 \\ \vdots & \ddots & \ddots & \vdots \\ -2 p & \ldots & -2 p & -p\end{array}\right]$

The next step is to go back to Equation (5.17), where the underlying control trajectory $\dot{u}(\tau)$ is defined by a set of exponentially decaying functions within $0 \leq \tau \leq T_{p}$ using Equation (5.18), which guarantees the optimal convergence as follows:

$$
\dot{u}(\tau)=-K_{m p c} e^{\left(A-B K_{m p c}\right) \tau} x\left(t_{i}\right)
$$

As the state variable $x(t)$ consists of two components, $\dot{x}_{m}(t)$ and $y(t)$, we can write 


$$
\dot{u}(\tau)=-\left[K_{x} K_{y}\right] e^{\left(A-B\left[K_{x} K_{y}\right]\right) \tau} x\left(t_{i}\right)
$$

Moreover, this trajectory should also satisfy the following equation for the system to be $L^{2}$ stable:

$$
\lim _{T_{p} \rightarrow \infty} \int_{0}^{T_{p}} \dot{u}(\tau)^{2} d \tau<\infty
$$

Wang (2009) states that if there is an external constant input signal to the control system, such as a set-point signal or a constant disturbance signal, the control signal itself does not satisfy this property. That is the main reason why the continuous-time predictive control design will be used to target the derivative of the control signal, which satisfies the property given by Equation (5.28).

For this class of systems, the convergence of the derivative to zero is guaranteed for a given $p>0$ and some small $\varepsilon$ by the following inequality based on the orthonormal property of the Laguerre functions:

$$
\int_{0}^{\infty}\left(\dot{u}(\tau)-\sum_{i=1}^{N} \xi_{i} l_{i}(\tau)\right)^{2}<\varepsilon
$$

Finally, we describe the derivative of the control signal in terms of an orthonormal expansion using Laguerre functions based on the series expansion as follows:

$$
\dot{u}(\tau)=\sum_{i=1}^{N} \xi_{i} l_{i}(\tau)=L(\tau)^{T} \eta
$$

where $\eta=\left[\xi_{1} \xi_{2} \ldots \xi_{N}\right]^{T}$ is a vector of coefficients, and $L(\tau)$ are the set of orthonormal basis functions. 
Now, it is appropriate to model the future predicted trajectory of our inventory levels using this information. Assuming the initial state information $x\left(t_{i}\right)$ is available, and given by the equation $\dot{x}\left(t_{i}\right)=e^{A \tau} x\left(t_{i}\right)$, the predicted inventory level state variable at a future time $t_{i}+\tau$ is obtained by the following equation:

$$
x\left(t_{i}+\tau\right)=e^{A \tau} x\left(t_{i}\right)+\int_{0}^{\tau} e^{A(\tau-\gamma)} B \dot{u}(\gamma) d \gamma
$$

where $t_{i}<\tau<t_{i}+T_{p}$, and $\gamma$ is a time parameter specifically used for the integration, $0<\gamma<\tau$. Next, using Equation (5.30), we obtain the control signal for each $i, i=1, \ldots, m$ of the control vector $\dot{u}(\tau)$ as $\dot{u}_{i}(\tau)=L_{i}(\tau)^{T} \eta_{i}$. We define $p_{i}$ and $N_{i}$ for each Laguerre element $L_{i}(\tau)$. Using the vector representation for the components of Equation (5.30) and Equation $(5.31) \quad$ as $\quad B=\left[B_{1} B_{2}, \ldots, B_{m}\right], \quad L_{i}(\tau)^{T}=\left[l_{1}^{i}(\tau) l_{2}^{i}(\tau) \ldots l_{N_{i}}^{i}(\tau)\right], \quad$ and $\eta_{i}=\left[\xi_{1}^{i} \xi_{2}^{i} \ldots \xi_{N_{i}}^{i}\right]^{T}$, we obtain the following equation:

$$
\begin{aligned}
x\left(t_{i}+\tau\right)=e^{A \tau} x\left(t_{i}\right) & +\int_{0}^{\tau} e^{A(\tau-\gamma)}\left[B_{1} L_{1}(\gamma)^{T} \ldots B_{m} L_{m}(\gamma)^{T}\right] d \gamma \eta \\
& =e^{A \tau} x\left(t_{i}\right)+\Gamma(\tau)^{T} \eta
\end{aligned}
$$

where the convolution integral is $\Gamma(\tau)^{T}=\int_{0}^{\infty} e^{A(\tau-\gamma)}\left[B_{1} L_{1}(\gamma)^{T} \ldots B_{m} L_{m}(\gamma)^{T}\right] d \gamma \quad$ with a dimension of $n\left(\sum_{i=1}^{m} N_{i}\right)$, and $\eta=\left[\begin{array}{lll}\eta_{1} & \eta_{2} \ldots \eta_{m}\end{array}\right]$ with a dimension of $\sum_{i=1}^{m} N_{i}$. Then we can obtain the predicted output inventory levels for emergency supplies as follows:

$$
y\left(t_{i}+\tau\right)=C e^{A \tau} x\left(t_{i}\right)+C \Gamma(\tau)^{T} \eta
$$


The difficulty comes from the calculation of the convolution integral. Using the matrix differentiation in terms of $\int e^{A(\tau-\gamma)} d \gamma=-A^{-1} e^{A(\tau-\gamma)}$, we can write the following:

$$
\Gamma_{i}(\tau)^{T}=-A^{-1} \int_{0}^{\tau} d\left(e^{A(\tau-\gamma)}\right) B L_{i}(\gamma)^{T}
$$

By taking the matrix $A$ to the left-hand side, applying the method of integration by parts, and using the equation $L(\tau)=e^{A_{p} \tau} L(0)$, we obtain the following equation:

$$
\begin{gathered}
A \Gamma_{i}(\tau)^{T}=-\left[B_{i} L_{i}(\gamma)^{T}-e^{A \tau} B L_{i}(0)^{T}\right]+\int_{0}^{\tau}\left(e^{A(\tau-\gamma)}\right) B_{i} L_{i}(\gamma)^{T} A_{p}^{T} d \gamma \\
=-\left[B_{i} L_{i}(\gamma)^{T}-e^{A \tau} B L_{i}(0)^{T}\right]+\Gamma_{i}(\tau)^{T} A_{p}^{T} \\
\text { where } A_{p}=\left[\begin{array}{cccc}
-p & 0 & \ldots & 0 \\
-2 p & -p & \ldots & 0 \\
\vdots & \ddots & \ddots & \vdots \\
-2 p & \ldots & -2 p & -p
\end{array}\right] \text { given in Equation (5.25). Generalizing this for the }
\end{gathered}
$$

Laguerre vector $L(\tau)$, the computation can be performed through the following equation for each individual component $i, 1 \leq i \leq m$, for a given $\tau$ :

$$
A \Gamma_{i}(\tau)^{T}-\Gamma_{i}(\tau)^{T} A_{p}^{T}=-B_{i} L_{i}(\tau)^{T}+e^{A \tau} B_{i} L_{i}(0)^{T}
$$

where we use the orthonormal Laguerre equality of Equation (5.18), integration by parts, and the convolution integral $\Gamma(\tau)$. The solution of this equation requires solving $n \times N$ linear equations where the components of $\Gamma(\tau)$ can be computed systematically for each element $i, 1 \leq i \leq m$ as follows:

$$
(A+p I) \Gamma_{i}(\tau)^{T}=-B_{i} L_{i}(\tau)^{T}+e^{A \tau} B_{i} L_{i}(0)^{T}-2 p I \sum_{k=1}^{i} \Gamma_{k}(\tau)^{T}, i=1, \ldots, N
$$


With these numerical calculations in terms of decomposing $n \times N$ linear equations into $N$ separate solutions of $n$ linear equations, it is possible to obtain the desired inventory state vector using Equation (5.37).

\subsubsection{Cost Function}

For a given target inventory value (set point signal) $r\left(t_{i}\right)$, the objective of our predictive control system is to bring the predicted output inventory level $y\left(t_{i}\right)$ as close as possible to the target set-point signal within the prediction horizon $T_{p}$. This objective can be equally stated as to find the best control parameter vector $u\left(t_{i}\right)$ such that an error function between the target and the predicted output inventory level is minimized.

Traditional predictive control models use the cost function based on the squares of the system states and the controls. In our continuous time predictive control model, however, the objective is to minimize the following cost function within the moving horizon window:

$$
J=\int_{0}^{T_{p}}\left(x\left(t_{i}+\tau\right)^{T} Q\left(x\left(t_{i}+\tau\right)+\dot{u}(\tau)^{T} R \dot{u}(\tau)\right) d \tau\right.
$$

where $Q>0$ and $R>0$ are weight matrices. The reason for using the derivative of the control in Equation (5.38) comes from the fact that the control signal itself does not satisfy the property given by Equation (5.28) and therefore cannot be used as Laguerre functions to satisfy the convergence within the model.

Here, the important point is how to incorporate the inventory planning set point signals (target inventory levels) into the cost function. We obtain these target inventory 
levels using the multi-commodity SHIC model (Ozguven and Ozbay, 2011) and give them as target signals $r\left(t_{i}\right)$ to the online predictive control framework. Then the cost function becomes:

$$
J=\int_{0}^{T_{p}}\left(\left(r\left(t_{i}\right)-y\left(t_{i}+\tau\right)\right)^{T} Q\left(r\left(t_{i}\right)-y\left(t_{i}+\tau\right)\right)+\dot{u}(\tau)^{T} \operatorname{R} \dot{u}(\tau)\right) d \tau
$$

Therefore, without any constraint, the objective is to find the control vector that will determine the inventory level $y\left(t_{i}+\tau\right)$ as close as possible to the future trajectory of the set point target inventory levels. Based on this information, our augmented model becomes:

$$
\begin{gathered}
{\left[\begin{array}{c}
\ddot{x}_{m}\left(t_{i}+\tau\right) \\
\dot{y}\left(t_{i}+\tau\right)-r\left(\mid t_{i}\right)
\end{array}\right]=A\left[\begin{array}{c}
\dot{x}_{m}\left(t_{i}+\tau\right) \\
y\left(t_{i}+\tau\right)-r\left(\mid t_{i}\right)
\end{array}\right]+B \dot{u}(\tau)} \\
y(\tau)=C\left[\begin{array}{c}
\dot{x}_{m}\left(t_{i}+\tau\right) \\
y\left(t_{i}+\tau\right)-r\left(\mid t_{i}\right)
\end{array}\right]
\end{gathered}
$$

By choosing $Q=C^{T} C$, we can write the cost function as in Equation (5.38), where the initial inventory level state information contains the error $y\left(t_{i}\right)-r\left(t_{i}\right)$ instead of only $y\left(t_{i}\right)$. Moreover, we choose $R$ as a diagonal matrix as $R=\left[\begin{array}{cccc}r_{1} & \ldots & \ldots & 0 \\ \vdots & \ddots & \ddots & \vdots \\ \vdots & \ddots & \ddots & \vdots \\ 0 & \ldots & \ldots & r_{k}\end{array}\right], k=1, \ldots, m$ so that we can write the following equality:

$$
\left.\int_{0}^{T_{p}} \dot{u}(\tau)^{T} R \dot{u}(\tau)\right) d \tau=\sum_{i=1}^{m} \int_{0}^{T_{p}} r_{k} \dot{u}_{k}(\tau)^{2} d \tau
$$

In our inventory management framework, we want to set the prediction horizon $T_{p}$ as large as possible to allow the convergence of the derivative of the control to zero 
when $\tau \geq T_{p}$. Selecting $T_{p}$ larger than the time for which the control signal is effective and using the orthonormal properties of Laguerre functions where $\int_{0}^{\infty} L_{k}(\tau) L_{k}(\tau)^{T}$ is equal to the identity matrix for the $k t h$ input, we get the following:

$$
\left.\int_{0}^{T_{p}} \dot{u}_{k}(\tau)^{T} \dot{u}_{k}(\tau)\right) d \tau \approx \int_{0}^{\infty} \eta_{k}^{T} L_{k}(\tau) L_{k}(\tau)^{T} \eta_{k} d \tau=\eta_{k}^{T} \eta_{k}
$$

With this information, finally, we can write the cost function as follows:

$$
J=\int_{0}^{T_{p}}\left(\left(r\left(t_{i}\right)-y\left(t_{i}+\tau\right)\right)^{T} Q\left(r\left(t_{i}\right)-y\left(t_{i}+\tau\right)\right)+\eta^{T} R_{L} \eta\right.
$$

Here, $R_{L}$ is a block diagonal matrix where the $k t h$ block is $R_{k}=r_{k} I_{N_{k} \times N_{k}}$.

Substituting the state variable information for $x\left(t_{i}+\tau\right)=r\left(t_{i}\right)-y\left(t_{i}+\tau\right)$ given in Equation (5.32) into our objective function yields the following:

$$
\begin{gathered}
J=\int_{0}^{T_{p}}\left(e^{A \tau} x\left(t_{i}\right)+\Gamma(\tau)^{T} \eta\right) Q\left(e^{A \tau} x\left(t_{i}\right)+\Gamma(\tau)^{T} \eta\right) d \tau+\eta^{T} R_{L} \eta \\
=\eta^{T}\left[\int_{0}^{T_{p}} \Gamma(\tau) Q \Gamma(\tau)^{T} d \tau+R_{L}\right] \eta+2 \eta^{T} \int_{0}^{T_{p}} \Gamma(\tau) Q e^{A \tau} d \tau x\left(t_{i}\right)+x\left(t_{i}\right)^{T} \int_{0}^{T_{p}} e^{A^{T} \tau} Q e^{A \tau} d \tau x\left(t_{i}\right)
\end{gathered}
$$

Here, for the sake of simplicity, let's define $H=\left[\int_{0}^{T_{p}} \Gamma(\tau) Q \Gamma(\tau)^{T} d \tau+R_{L}\right]$ and $F=\int_{0}^{T_{p}} \Gamma(\tau) Q e^{A \tau} d \tau$ so that our objective function for the minimization finally becomes as follows:

$$
J=\eta^{T} H \eta+2 \eta^{T} F x\left(t_{i}\right)+x\left(t_{i}\right)^{T} \int_{0}^{T_{p}} e^{A^{T} \tau} Q e^{A \tau} d \tau x\left(t_{i}\right)
$$




\subsubsection{Constraints}

All real-world control systems have to deal with constraints at some point, which is exactly the case for emergency relief control systems. Given our quadratic objective function, we want to introduce constraints due to disruptions in emergency relief operations such as demand fluctuations in shelters, damaged transportation networks, limited supply and deliveries, etc., and then solve the final quadratic programming problem. Therefore, we have three types of constraints that we impose on our framework.

First of all, we may always have limitations on our inventory levels of emergency commodities during relief operations. To be able to reflect this situation in our model, we can impose lower and higher bounds for output inventory levels using Equation (5.33) as:

$$
y_{\min } \leq y\left(t_{i}+\tau\right)=C\left[e^{A \tau} x\left(t_{i}\right)+\Gamma(\tau)^{T} \eta\right] \leq y_{\max }
$$

However, it is important to note that output constraints could cause a predictive control system to become unstable (Wang, 2009); therefore, one should be cautious while implementing such constraints.

Constraints of the second type include the upper and lower limits for the control signals. Note that controls allow us to regulate the flow of vital supplies for inventories so that we can always restrain the amount of emergency supply movement within our model. This notion introduces limiting constraints on the control signals, which are mostly present in real-world emergency relief systems. That is, in our emergency management framework, these limiting constraints exist due to the characteristics of the flow of emergency supplies, congested flows due to limited transportation networks, or 
limited shelter and inventory capacities. The constraints for the control signals are in the form of:

$$
u_{\min }\left(t_{i}+\tau_{i}\right) \leq\left[\begin{array}{cccc}
\int_{0}^{\tau_{i}} L_{1}(\gamma)^{T} d \gamma & 0 & \ldots & 0 \\
\vdots & \int_{0}^{\tau_{i}} L_{2}(\gamma)^{T} d \gamma & \ddots & 0 \\
\vdots & \ddots & \ddots & \vdots \\
0 & 0 & \ldots & \int_{0}^{\tau_{i}} L_{r}(\gamma)^{T} d \gamma
\end{array}\right] \eta+u\left(t_{i-1}\right) \leq u_{\max }\left(t_{i}+\tau_{i}\right)
$$

where $\tau_{i}, i=1, \ldots, K$ denotes the set of future time instants (due to discretization for constraints) at which we impose limits on the control signal, and $L_{k}(\tau), k=1, \ldots, r$ are the Laguerre functions. These constraints also account for the effect of the stochasticity involved within our emergency framework, due to disruptions in the transportation network or drastic changes in demand and deliveries, etc. To be able to evaluate these constraints, we focus on the time parameter $\tau_{i}, i=1, \ldots, K$, for which the following equation holds:

$$
u\left(\tau_{i}\right)=u\left(t_{i}\right)+\int_{0}^{\tau_{i}} \dot{u}(\gamma) d \gamma=u\left(t_{i}\right)+\int_{0}^{\tau_{i}} L(\gamma)^{T} \eta d \gamma=u\left(t_{i}\right)+\left(L\left(\tau_{i}\right)^{T}-L(0)^{T}\right) A_{p}^{-T} \eta
$$

With the information on $u\left(t_{i}\right)$, we can write this as:

$$
u\left(\tau_{i}\right)=u\left(t_{i}\right)+C_{u} \eta
$$

where we define $C_{u}=\left(L\left(\tau_{i}\right)^{T}-L(0)^{T}\right) A_{p}^{-T}$ so that our constraint becomes:

$$
u_{\min }\left(t_{i}+\tau_{i}\right) \leq C_{u} \eta+u\left(t_{i}\right) \leq u_{\max }\left(t_{i}+\tau_{i}\right)
$$


Constraints of the third type are imposed on the derivative of the control that basically represents the rate of change in the control signals. This is mathematically straightforward, as the optimal control solution is based on the convergence of the derivative of the control. In terms of our emergency management framework, we try to constrain the rate of change of the emergency supply flow. The disruptions and the uncertainties within the emergency relief system can cause sudden rate changes in the controls, but our controller should account for this using the following equations:

$$
\dot{u}_{\text {min }}\left(t_{i}+\tau_{i}\right) \leq\left[\begin{array}{cccc}
L_{1}\left(\tau_{i}\right)^{T} & 0 & \ldots & 0 \\
\vdots & L_{2}\left(\tau_{i}\right)^{T} & \ddots & 0 \\
\vdots & \ddots & \ddots & \vdots \\
0 & 0 & \ldots & L_{r}\left(\tau_{i}\right)^{T}
\end{array}\right] \eta \leq \dot{u}_{\max }\left(t_{i}+\tau_{i}\right)
$$

where $\tau_{i}, i=1, \ldots, K$ denotes the set of future time instants at which we impose limits on the derivative of the control signal, and $L_{k}(\tau), k=1, \ldots, r$ are the Laguerre functions that guarantee the convergence of the derivative to zero.

\subsubsection{SHIC Integration}

We use the SHIC model first developed by Ozbay and Ozguven (2007) within our online continuous time model predictive model for two significant purposes:

- The set point signals (target inventory stock levels) are calculated via the multicommodity SHIC model for each specific case and are fed into our Quadratic Inventory Control Problem (QICP).

- The state-space matrix elements are defined using the consumption and delivery distributions calculated using the multi-commodity SHIC model. 
That is, whenever there is a change in the disaster inventory demand of victims located in shelters, we calculate new target safety stocks and new consumption and delivery distributions via the multi-commodity version of the SHIC model, and we feed these values into the online continuous model predictive model to predict the new future trajectories given the current inventory levels.

\subsubsection{Solving the Quadratic Inventory Control Problem (QICP)}

With the cost function and inequality constraints given in the previous subsections, we can obtain the proposed Quadratic Inventory Control Problem (QICP) as:

$$
\begin{aligned}
& J=\eta^{T} H \eta+2 \eta^{T} F x\left(t_{i}\right)+x\left(t_{i}\right)^{T} \int_{0}^{T_{p}} e^{A^{T} \tau} Q e^{A \tau} d \tau x\left(t_{i}\right) \\
& \text { subject to } \\
& y_{\min } \leq y\left(t_{i}+\tau\right) \leq y_{\max } \\
& u_{\text {min }}\left(t_{i}+\tau_{i}\right) \leq C_{u} \eta+u\left(t_{i}\right) \leq u_{\text {max }}\left(t_{i}+\tau_{i}\right) \\
& \dot{u}_{\text {min }}\left(t_{i}+\tau_{i}\right) \leq L_{u} \eta \leq \dot{u}_{\text {max }}\left(t_{i}+\tau_{i}\right) \\
& H=\left[\int_{0}^{T_{p}} \Gamma(\tau) Q \Gamma(\tau)^{T} d \tau+R_{L}\right] \\
& F=\int_{0}^{T_{p}} \Gamma(\tau) Q e^{A \tau} d \tau \\
& \Gamma(\tau)^{T}=\int_{0}^{\infty} e^{A(\tau-\gamma)}\left[B_{1} L_{1}(\gamma)^{T} \ldots B_{m} L_{m}(\gamma)^{T}\right] d \gamma, \\
& L_{i}(\tau)^{T}=\left[l_{1}^{i}(\tau) l_{2}^{i}(\tau) \ldots l_{N_{i}}^{i}(\tau)\right]
\end{aligned}
$$




$$
\begin{aligned}
& C_{u}=\left[\begin{array}{cccc}
\int_{0}^{\tau_{i}} L_{1}(\gamma)^{T} d \gamma & 0 & \ldots & 0 \\
\vdots & \int_{0}^{\tau_{i}} L_{2}(\gamma)^{T} d \gamma & \ddots & 0 \\
\vdots & \ddots & \ddots & \vdots \\
0 & 0 & \ldots & \int_{0}^{\tau_{i}} L_{r}(\gamma)^{T} d \gamma
\end{array}\right] \text {, and } \\
& L_{u}=\left[\begin{array}{cccc}
L_{1}\left(\tau_{i}\right)^{T} & 0 & \ldots & 0 \\
\vdots & L_{2}\left(\tau_{i}\right)^{T} & \ddots & 0 \\
\vdots & \ddots & \ddots & \vdots \\
0 & 0 & \ldots & L_{r}\left(\tau_{i}\right)^{T}
\end{array}\right]
\end{aligned}
$$

We will attempt to solve the above quadratic programming problem with an efficient cyclic coordinate search method, namely the method of Hildreth and D'Espo (Wismer and Chattergy, 1978). First, we have to obtain the dual of our problem. Let's simplify our QICP problem with respect to our decision vector $\eta$ as follows:

$$
\begin{gathered}
J=\frac{1}{2} \eta^{T} H \eta+\eta^{T} F x\left(t_{i}\right)+\frac{1}{2} x\left(t_{i}\right)^{T} \int_{0}^{T_{p}} e^{A^{T} \tau} Q e^{A \tau} d \tau x\left(t_{i}\right) \\
\text { subject to } \\
\Theta \eta \leq v
\end{gathered}
$$

where $\Theta$ and $v$ represent compatible matrices and vectors for the constraints of the quadratic programming problem. Here, we will make use of Lagrangian multipliers to rewrite the objective function as an equivalent formulation:

$$
J=\max _{\lambda \geq 0} \min _{\eta}\left[\frac{1}{2} \eta^{T} H \eta+\eta^{T} F x\left(t_{i}\right)+\frac{1}{2} x\left(t_{i}\right)^{T} \int_{0}^{T_{p}} e^{A^{T} \tau} Q e^{A \tau} d \tau x\left(t_{i}\right)+\lambda^{T}(\Theta \eta-v)\right]
$$

This function can be minimized by solving for $\eta$. Using the method of Lagrangian multipliers, the optimal $\eta$ that minimizes this objective function can be 
obtained as $\eta=-H^{-1}\left(F x\left(t_{i}\right)+\Theta^{T} \lambda\right)$. However, we still do have the constraint set to consider. Therefore, we will focus on the constraints in terms of active and inactive ones. For instance, $\Theta \eta=v$ represents the active constraint set where $\Theta \eta<v$ gives the inactive constraints for our QICP problem. Here, we will define the well-known KarushKuhn-Tucker (KKT) conditions for the model in terms of the Lagrange multipliers with the matrix representation, $\lambda$, as follows:

$$
\begin{gathered}
H \eta+F x\left(t_{i}\right)+\Theta^{T} \lambda=0 \\
\Theta \eta-v \leq 0 \\
\lambda^{T}(\Theta \eta-v)=0 \\
\lambda \geq 0
\end{gathered}
$$

These conditions can be re-written in terms of the set of active constraints (say, kth row represents the active constraint row for constraint matrix $\Theta$ ) as:

$$
\begin{gathered}
H \eta+F x\left(t_{i}\right)+\sum_{k: \text { active }} \lambda_{k} \Theta_{k}^{T}=0 \\
\Theta_{k} \eta-v_{k}=0, k: \text { active } \\
\Theta_{k} \eta-v_{k}<0, k: \text { inactive } \\
\lambda_{k} \geq 0, k: \text { active } \\
\lambda_{k}=0, k \text { : inactive }
\end{gathered}
$$

Now that we have the KKT conditions, we can obtain the dual problem by substituting $\eta=-H^{-1}\left(F x\left(t_{i}\right)+\Theta^{T} \lambda\right)$ into Equation (5.54) as:

$$
J_{d}=\max _{\lambda \geq 0}\left[-\frac{1}{2} \lambda^{T} E \lambda-\lambda^{T} M-\frac{1}{2} F^{T} x\left(t_{i}\right)^{T} H^{-1} F x\left(t_{i}\right)\right]
$$

where $E=\Theta H^{-1} \Theta^{T}$ and $M=v+\Theta H^{-1} F x\left(t_{i}\right)$. Finally, changing this formulation into a minimization problem, we obtain our finalized dual problem where $\lambda$ is our decision variable: 


$$
J_{d}=\min _{\lambda \geq 0}\left[\frac{1}{2} \lambda^{T} E \lambda+\lambda^{T} M+\frac{1}{2} F^{T} x\left(t_{i}\right)^{T} H^{-1} F x\left(t_{i}\right)\right]
$$

To solve this model, we will use the method of Hildreth and D'Espo (see Wismer and Chattergy, 1978 for details). The procedure is as follows:

Step 0: Select an initial $\lambda \geq 0$ and focus on the components $\lambda_{k}$ so that $\lambda_{k} \geq 0$.

Step 1: Let $j=1$ and $\lambda_{k}^{j}=\lambda_{0}$.

Step 2: Minimize the quadratic objective function with respect to $\lambda_{k}^{j}$ by adjusting $\lambda_{k}^{j}$, If $\lambda_{k}^{j}$ becomes smaller than zero, then fix as $\lambda_{k}^{j}=0$. The adjustment procedure for one iteration $j$ is:

$$
\begin{gathered}
\lambda_{k}^{j+1}=\max \left(0, w_{k}^{j+1}\right) \\
\text { where } \\
w_{k}^{j+1}=-\frac{1}{e_{k k}}\left[s_{k}+\sum_{i=1}^{k-1} e_{k j} \lambda_{i}^{m+1}+\sum_{i=k+1}^{n} e_{k j} \lambda_{i}^{m}\right]
\end{gathered}
$$

Here, $e_{k j}$ represents the kjth element of the matrix $E=\Theta H^{-1} \Theta^{T}$, and $s_{k}$ is the $k t h$ element of the vector $M=v+\Theta H^{-1} F x\left(t_{i}\right)$.

Step 3: Let $j=j+1$. Check for the convergence criteria where the maximum iteration number is $N_{\max }$. If $k \leq N_{\max }$, go to Step 2 .

Step 4: If $\lambda_{k}^{j} \neq \lambda_{k}^{j+1}$, then let $\lambda=\lambda_{k}^{j}$, and go to Step 1. Otherwise, stop the iterations so that we have $\lambda_{k}^{j}=\lambda_{k}^{j+1}=\lambda_{k}^{j^{*}}$. 
Here, if and only if the active constraints are linearly independent and the number of active constraints is smaller than or equal to the number of decision variables, then the dual problem will converge to the optimum solution set (Iusem and De Pierro, 1990).

This algorithm does not include any matrix inversion, and it reaches a nearoptimal solution even if there is an ill-conditioned situation due to the constraints. This indicates that the technique is very powerful for our real-time inventory management framework, as it can recover from an ill-conditioned constrained problem (Wang, 2010). As a result of the algorithm, we obtain the Lagrangian set of $\lambda^{*}$ that contains zeros for inactive constraints and positive values for active constraints, as follows:

$$
\begin{gathered}
\lambda_{\text {active }}^{*}=-\left(\Theta_{\text {active }} H^{-1} \Theta_{\text {active }}^{T}\right)^{-1}\left(v_{\text {active }}+\Theta_{\text {active }} H^{-1} F x\left(t_{i}\right)\right) \\
\lambda_{\text {inactive }}^{*}=0
\end{gathered}
$$

where $\Theta_{\text {active }}$ and $v_{\text {active }}$ represent compatible matrices and vectors after deleting the zero row elements of the inactive components of the Lagrangian set. The proof of convergence for the algorithm is shown by Wismer and Chattergy (1978) based on the existence of $\left(\Theta_{\text {active }} H^{-1} \Theta_{\text {active }}^{T}\right)^{-1}$.

Finally, after presenting the solution algorithm, we will make use of the equality $\eta=-H^{-1}\left(F x\left(t_{i}\right)+\Theta^{T} \lambda\right)$ to obtain $K_{m p c}$, and we will substitute it into $\dot{u}(\tau)=L(\tau)^{T} \eta$ to obtain the following:

$$
\dot{u}(\tau)=L(\tau)^{T}\left[-H^{-1}\left(F x(\tau)+\Theta^{T} \lambda\right)\right]
$$

where $K_{m p c}$ can be calculated as follows:

$$
K_{m p c}=L(\tau)^{T} H^{-1} F
$$




\subsubsection{Observer}

As real-time inventory states cannot be measured via the SHIC model, the prediction of future emergency system behavior is based on the original inventory state variable $x_{m}(t)$ using the derivative of the inventory state variable, namely $\dot{x}(t)$, in the augmented state-space model. This derivative operation will be obtained using a filter as the continuous time observer. This observer will not only attain the inventory state variable information, but also allow filtering of the measurement noise in the emergency relief system. The observer equation for our continuous time model predictive control methodology is as follows:

$$
\frac{d \widehat{x}(t)}{d t}=A \dot{x}(t)+B \dot{u}(t)+K_{o b s}(y(t)-C \widehat{x}(t))
$$

where $\hat{x}(t)$ is the estimate of $x(t), K_{o b s}$ is the observer gain, $A, B, C$ are the augmented state-space matrices aforementioned, and $\dot{u}(t)$ is the derivative control signal obtained from the optimal solution of the overall model predictive control problem. $K_{o b s}$ is directly affected by the disturbances in the emergency relief system (in the presence of noise); therefore, filters such as Kalman filters can be used effectively to estimate the inventory state variable and obtain the gain matrix (Anderson and Moore, 1979).

The overall model with the observer can be seen in Figure 5.5. 


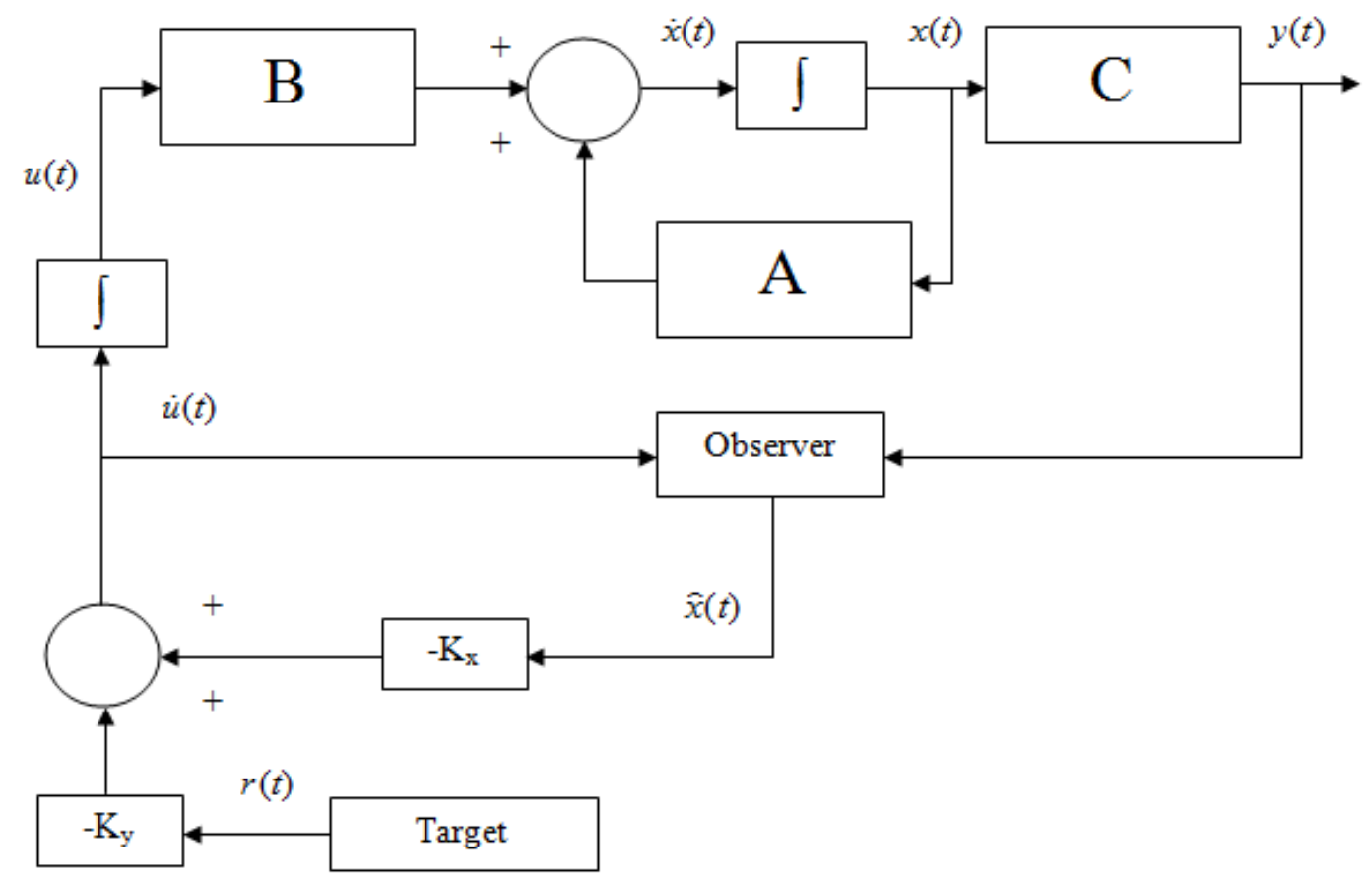

Figure 5.5 Emergency Inventory Control Framework with the Observer

\subsubsection{Stochastic Noise}

We introduce stochastic noise into the system to better simulate the extreme complexity expected under real-world emergency conditions in the aftermath of a disaster. We define the stochastic noise $\omega(t)$ as a white noise vector where its mean is defined as $E\{\omega(t)\}=0$, and the correlation matrix of the noise is defined as $E\left\{\omega(t) \omega(t)^{T}\right\}=\sigma^{2} I$. Within the case scenarios studied in the following subsections, white Gaussian noise is added to the system using the signal-to-noise ratio (SNR) measure (based on the ratio of the signal to the noise) to observe the performance of the model under stochastic conditions. 


\subsubsection{Model Tuning Guidelines}

The selection of design variables has utmost importance to achieve a reliable model. These variables include $p$, the pole location parameter; $N$, the number of terms used in the Laguerre system to capture the control signal; the prediction horizon $T_{p}$; and the weight matrices $Q$ and $R$. Pole location parameter $p$ has great importance for the prediction of the control horizon. The most effective tuning procedure for this parameter is to start close to the location of the system dominant pole, and to then change the parameter slowly to gain the optimum performance (Wang, 2001). The prediction horizon, on the other hand, is selected to be $T_{p}=\frac{10}{p}$ to let the control signal effectively converge to a possible optimum value in our unstable and stochastic disaster environment. For the parameter $N$, the idea is to select a value that will produce desirable closed-loop responses. Again, for the unstable disaster conditions, $N$ is selected between $[1,5]$, which is found to be adequate for the control signal to achieve a stable convergence for such stochastic systems (Wang et al., 2008). Finally, the weight matrix $Q$ is selected to be equal to $Q=C^{T} C$ and $R$ as a variation of the identity matrix to achieve a better tuning procedure.

As a summary, input and outputs associated with the CMPC methodology are given in Table 5.5. 
Table 5.5 Inputs and Outputs for the CMPC Model

\begin{tabular}{|c|c|c|c|c|}
\hline $\begin{array}{l}\text { Inputs } \\
t_{k}: \text { Target Inventory Levels } \\
\text { obtained from SHIC Model } \\
\text { System State Information } \\
S N R \text { : White Gaussian noise } \\
\text { based on the coefficients of } \\
\text { variation } \\
\text { Constraint values on the } \\
\text { inventory levels, flow of supplies } \\
\text { and rate of flow of supplies. } \\
T_{p}: \text { Length of moving horizon } \\
\text { window } \\
p, N: \text { CMPC system parameters } \\
Q, R: \text { Weight matrices }\end{array}$ & $\Rightarrow$ & $\begin{array}{l}\text { CMPC } \\
\text { Model }\end{array}$ & $\Rightarrow$ & $\begin{array}{l}y_{k}: \text { Output inventory } \\
\text { levels } \\
u_{k}: \text { Output control } \\
\text { levels }\end{array}$ \\
\hline
\end{tabular}

\subsubsection{Case Studies}

Our initial aim is to determine the optimal safety stock levels in emergency inventories so that the consumption of stocked vital supplies at major shelters can occur with minimal disruption at the minimum cost. However, disruptions can always occur, as extreme events like disasters always come with high uncertainty and variability. For instance, the category of a disaster (in terms of severity) directly affects emergency relief operations. In a severe disaster such as a Category 5 hurricane, the demand of victims 
located in the shelters can be so high or chaotic that planning for high levels of emergency inventory may be needed. Possibly, due to disruption in the communications network, the supplier may not be informed about the actual demand; therefore, deliveries may be much lower than actual consumption in shelters, indicating the need for an initial safety stock level sufficient to serve the victims. Of course, such huge inventories will result in high inventory costs; therefore, an optimum way of determining the minimum inventory with the lowest probability of disruption is needed.

On the other hand, supply of a vital commodity may not be possible due to damaged or congested roadways, which in turn will increase the need for that commodity in shelters. Therefore, until the next delivery, shelters will need a sufficient safety stock to account for the amount of the commodity that cannot be delivered. Based on these, we apply our continuous time model predictive model to account for these stochasticities in our emergency management system. Within this methodology, the planning model results are used as set point signal inputs to an online model predictive control model. Using this information, the model predictive control approach (MPC) has the ability to compute the trajectory of the commodity inventory levels to optimize the future behavior of the disaster inventory management framework.

We formulate our model as analogous to a dynamic two-tank system (Almutairi and Zribi, 2006). This type of tank analogy has been successfully used for supply chain and inventory control model applications for many years; examples include Schwartz and Rivera (2010) and Wang et al. (2007). Our formulation for the two-commodity state space model is as follows: 


$$
\begin{gathered}
{\left[\begin{array}{c}
\dot{x}_{1}(t) \\
\dot{x}_{2}(t)
\end{array}\right]=\left[\begin{array}{cc}
-\frac{\beta_{1}}{\alpha_{1}} & \frac{\beta_{2}}{\alpha_{2}} \\
\frac{\beta_{1}}{\alpha_{1}} & -\frac{\beta_{2}}{\alpha_{2}}
\end{array}\right]\left[\begin{array}{l}
x_{1}(t) \\
x_{2}(t)
\end{array}\right]+\left[\begin{array}{cc}
\frac{\beta_{1}}{\alpha_{1}} & 0 \\
0 & \frac{\beta_{2}}{\alpha_{2}}
\end{array}\right] u(t)} \\
y(t)=\left[\begin{array}{ll}
1 & 0 \\
0 & 1
\end{array}\right]\left[\begin{array}{l}
x_{1}(t) \\
x_{2}(t)
\end{array}\right]
\end{gathered}
$$

where $\alpha_{1}>0$ and $\alpha_{2}>0$ represent the normalized and dimensionless system coefficients reflecting the dynamics (in terms of consumption) of the inventory levels of the commodities, and $\beta_{1}>0$ and $\beta_{2}>0$ are the normalized and dimensionless system coefficients reflecting the dynamics of the commodity deliveries. We obtain these coefficients from the SHIC model based on the coefficient of variations of base consumption and delivery distributions. Note that the coefficient of variation is simply the ratio of the standard deviation and mean of a probability distribution.

The next step is to introduce the observer. Using Equation (5.50), we introduce the observer into this model as follows:

$$
\left[\begin{array}{c}
\frac{d \widehat{x}_{1}(t)}{d t} \\
\frac{d \bar{x}_{2}(t)}{d t}
\end{array}\right]=\left[\begin{array}{cc}
-\frac{\beta_{1}}{\alpha_{1}} & \frac{\beta_{2}}{\alpha_{2}} \\
\frac{\beta_{1}}{\alpha_{1}} & -\frac{\beta_{2}}{\alpha_{2}}
\end{array}\right]\left[\begin{array}{l}
x_{1}(t) \\
x_{2}(t)
\end{array}\right]+\left[\begin{array}{cc}
-\frac{\beta_{1}}{\alpha_{1}} & 0 \\
0 & -\frac{\beta_{2}}{\alpha_{2}}
\end{array}\right] u(t)+K_{o b s}\left(\left[\begin{array}{l}
y_{1}(t) \\
y_{2}(t)
\end{array}\right]-\left[\begin{array}{ll}
1 & 0 \\
0 & 1
\end{array}\right]\left[\begin{array}{l}
\hat{x}_{1}(t) \\
\hat{x}_{2}(t)
\end{array}\right]\right)
$$

Given the characteristics of the stochastic disaster environment, for the case studies that will be presented in the following sections, the Kalman filter, proved to be efficient for systems that have noisy measurements (Anderson and Moore, 1979), will be used as the observer in Equation (5.65).

We build the case scenarios presented in the following sections on a time scale to make online tracking of supply flow possible where emergency officials can monitor the 
stock and flow of vital supplies every 6 hours after the occurrence of a disaster. This is consistent with the response of the New Jersey State Emergency Operations Center, which monitored the progress of relief operations during Hurricane Irene, where monitoring occurred every 4-7 hours in terms of evacuation details, roadway disruptions, shelter locations, resources and supply details, as presented at the New Jersey Office of Emergency Management web site (http://njoem.posterous.com/situation-report).

\subsubsection{Base Case}

Given the offline planning information for the emergency stocks of MRE's and medicine in Table 4.6, we focus on the online control of emergency supplies. Using the formulation in Equation (5.64), we will minimize the following problem (see Equation (5.52) for the original formulation):

$$
\begin{gathered}
J=\eta^{T} H \eta+2 \eta^{T} F\left[\begin{array}{ll}
x_{1} & x_{2}
\end{array}\right]+\left[\begin{array}{ll}
x_{1} & x_{2}
\end{array}\right]^{T} \int_{0}^{T_{p}} e^{A^{T} \tau} Q e^{A \tau} d \tau\left[x_{1} x_{2}\right] \\
\text { subject to } \\
0 \leq y_{1} \leq 10000 \text { (Capacity in terms of units of medicine) } \\
0 \leq y_{2} \leq 30000 \text { (Capacity in terms of units of MRE's) } \\
10000 \leq u_{1} \leq 50000 \text { (Limitations on the flow of medicine, units/6 hr) } \\
10000 \leq u_{1} \leq 30000 \text { (Limitations on the flow of MRE's, units/6 hr) } \\
-2000 \leq \dot{u}_{1} \leq 2000 \text { (Limitations on the rate of the medicine flow) } \\
-2000 \leq \dot{u}_{2} \leq 2000 \text { (Limitations on the rate of the MRE flow) }
\end{gathered}
$$

where

$$
\begin{aligned}
& {\left[\begin{array}{ll}
x_{1} & x_{2}
\end{array}\right]=\left[\begin{array}{ll}
r_{1} & \left.r_{2}\right]-\left[\begin{array}{ll}
y_{1} & y_{2}
\end{array}\right], \\
H=\left[\int_{0}^{T_{p}} \Gamma(\tau) Q \Gamma(\tau)^{T} d \tau+R_{L}\right],
\end{array}\right.}
\end{aligned}
$$




$$
\begin{aligned}
& F=\int_{0}^{T_{p}} \Gamma(\tau) Q e^{A \tau} d \tau, \\
& \Gamma(\tau)^{T}=\int_{0}^{\infty} e^{A(\tau-\gamma)}\left[B_{1} L_{1}(\gamma)^{T} \ldots B_{m} L_{m}(\gamma)^{T}\right] d \gamma, \\
& L_{i}(\tau)^{T}=\left[l_{1}^{i}(\tau) l_{2}^{i}(\tau) \ldots l_{N_{i}}^{i}(\tau)\right] \\
& C_{u}=\left[\begin{array}{cccc}
\int_{0}^{\tau_{i}} L_{1}(\gamma)^{T} d \gamma & 0 & \ldots & 0 \\
\vdots & \int_{0}^{\tau_{i}} L_{2}(\gamma)^{T} d \gamma & \ddots & 0 \\
\vdots & \ddots & \ddots & \vdots \\
0 & 0 & \ldots & \int_{0}^{\tau_{i}} L_{r}(\gamma)^{T} d \gamma
\end{array}\right] \text {, and } \\
& L_{u}=\left[\begin{array}{cccc}
L_{1}\left(\tau_{i}\right)^{T} & 0 & \ldots & 0 \\
\vdots & L_{2}\left(\tau_{i}\right)^{T} & \ddots & 0 \\
\vdots & \ddots & \ddots & \vdots \\
0 & 0 & \ldots & L_{r}\left(\tau_{i}\right)^{T}
\end{array}\right]
\end{aligned}
$$

The weight matrices for the model are selected as $Q=C^{T} C=\left[\begin{array}{ll}1 & 0 \\ 0 & 1\end{array}\right]^{T}\left[\begin{array}{ll}1 & 0 \\ 0 & 1\end{array}\right], R=0.1 I$, $p_{1}=p_{2}=0.5, T_{p_{1}}=10 / p_{1}, T_{p_{2}}=10 / p_{2}$, and $N_{1}=N_{2}=3$. We choose the set point signals from Table 4.6 for demand $u=5$, where $r_{\text {Medicine }}=r_{1}=6500$ units for the medicine and $r_{M R E s}=r_{2}=27000$ MRE's. We obtain the coefficients of variation for the specific case studied using the consumption and delivery distributions calculated via the offline planning model as $\frac{\beta_{1}}{\alpha_{1}}=0.323$ and $\frac{\beta_{2}}{\alpha_{2}}=0.255$ (Table 4.6). 
The results can be found in Figure 5.6 for 25 days (100 time steps) in the aftermath of the hurricane, where the stochasticity has not been introduced yet in terms of noise. Note that each time step represents 6 hours and there is no change in demand for vital supplies during the whole time period in this case. This analysis can be used for sudden onset disasters where the need for basic supplies is substantial in the initial periods after the disaster strikes. Here, the quadratic programming problem is solved with and without constraints to be able to observe the differences between the unconstrained and constrained conditions. Constrained conditions are very likely to occur during uncertain disaster conditions as the flow of emergency supplies to shelters may be limited due to disruptions in the transportation network and unavailability of personnel and vehicles, or due to the fact that some inventories may be lost. Therefore, the important point is to observe the behavior of the model with these constraints so that planners and decision makers can be aware of the time delay that can occur because of these disruptions and therefore can maintain a safety buffer for the vital supplies. As observed from Figure 5.6, it takes longer for the derivative of the control, control, and therefore output to converge to optimal levels when system constraints exist. It is very important to realize the time difference between the constrained and unconstrained analyses while reaching the target inventory stock levels. To further investigate this issue, we focus on the first 10 days (40 time steps) of Figure 5.6 to obtain Figure 5.7. It is clear that the system constraints due to the disaster conditions have a crucial effect on the convergence performance of the model. For this case, the model reacts accordingly to reach the optimal inventory stock levels within a couple of days without the constraints. The constrained case, however, indicates that it takes more time to adjust the flow of 
emergency supplies to account for the vital supplies needed at shelters. To account for this delay, one might consider increasing the safety stock on hand in the emergency inventory so that consumption can continue without disruption until delivery operations become more stable. However, note that the stochasticity is still not introduced into the system for this analysis.

Intuitively, it is clear from Figure 5.7 that real-life constraints on inventory levels and supply flows increase response time. If you have limitations on inventory levels, it takes longer to achieve the desired inventory levels as there are limitations on the stocks. On the other hand, constraints on the amount of deliveries also significantly affect the response time. Basically, imposing constraints on the system makes it difficult for the mathematical model to converge. Therefore, additional safety stock is needed to account for that need for vital supplies. Planners and decision makers should initially select the levels of safety stock accordingly to account for the time that can be lost due to these limitations.

Consider the example of the cyclone in Mozambique in 2007, where delivery of relief supplies to temporary camps for people affected by the disaster was not easy in the face of severe inventory capacity constraints (unavailability of locations to store commodities) (Amin et al., 2008). The National Emergency Operations Center maintained a registry of the temporary camps, and the delivery of daily supplies for these camps was coordinated very slowly. In this case, the proposed model can be used efficiently to analyze the disaster relief operations on the presence of such inventory level constraints. In order to solve this problem, the National Emergency Operations Center may decide on increasing the number of deliveries to the temporary camps as stocking 
commodities is substantially difficult. This can be achieved by increasing the rate of change using the third type of constraints. The logical way to do this is to increase the upper bound in the rate constraints and do not let the lower bound go below zero. This will gradually create an increase in the flow of supplies to satisfy the needs of victims located in temporary camps.

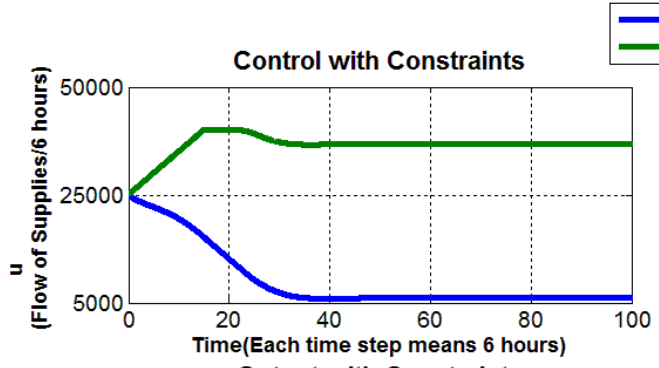
Output with Constraints
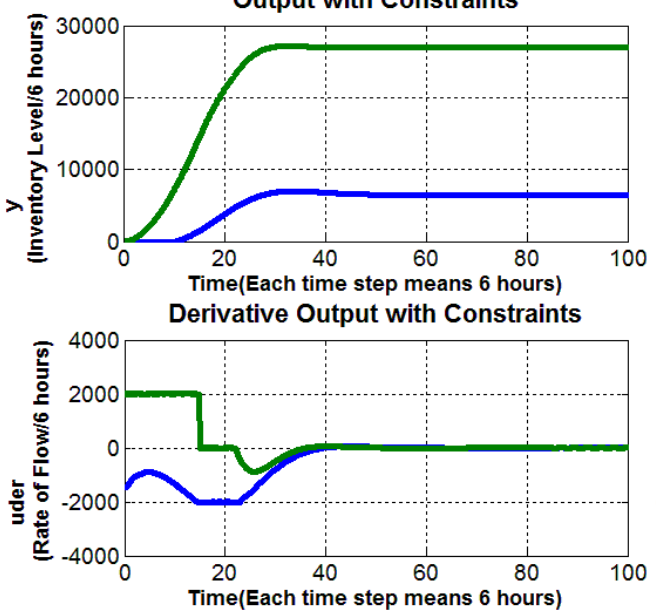

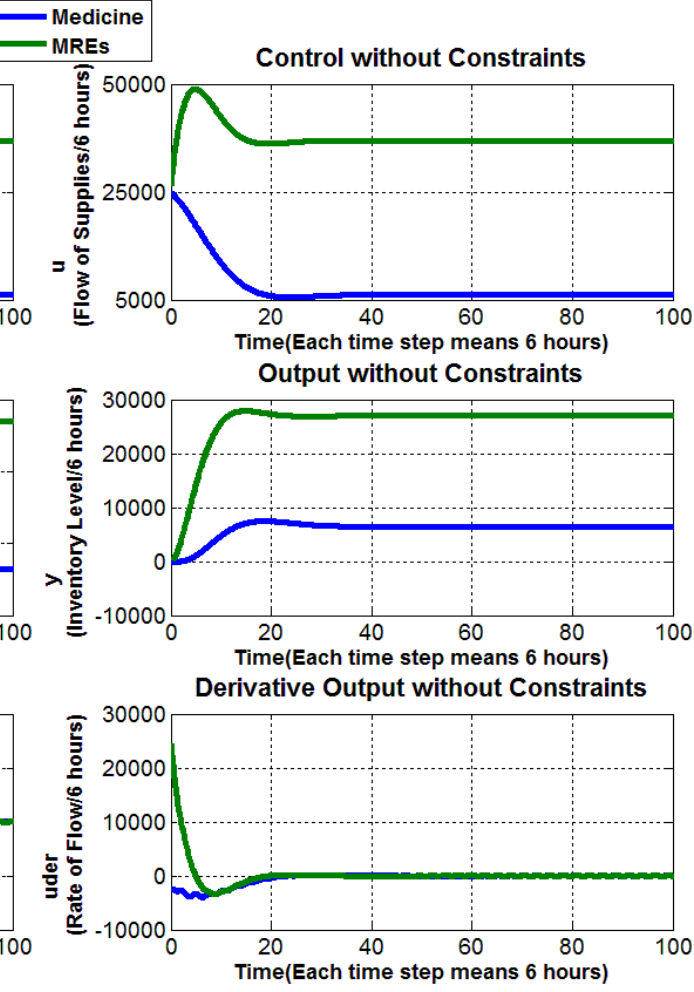

(a) 

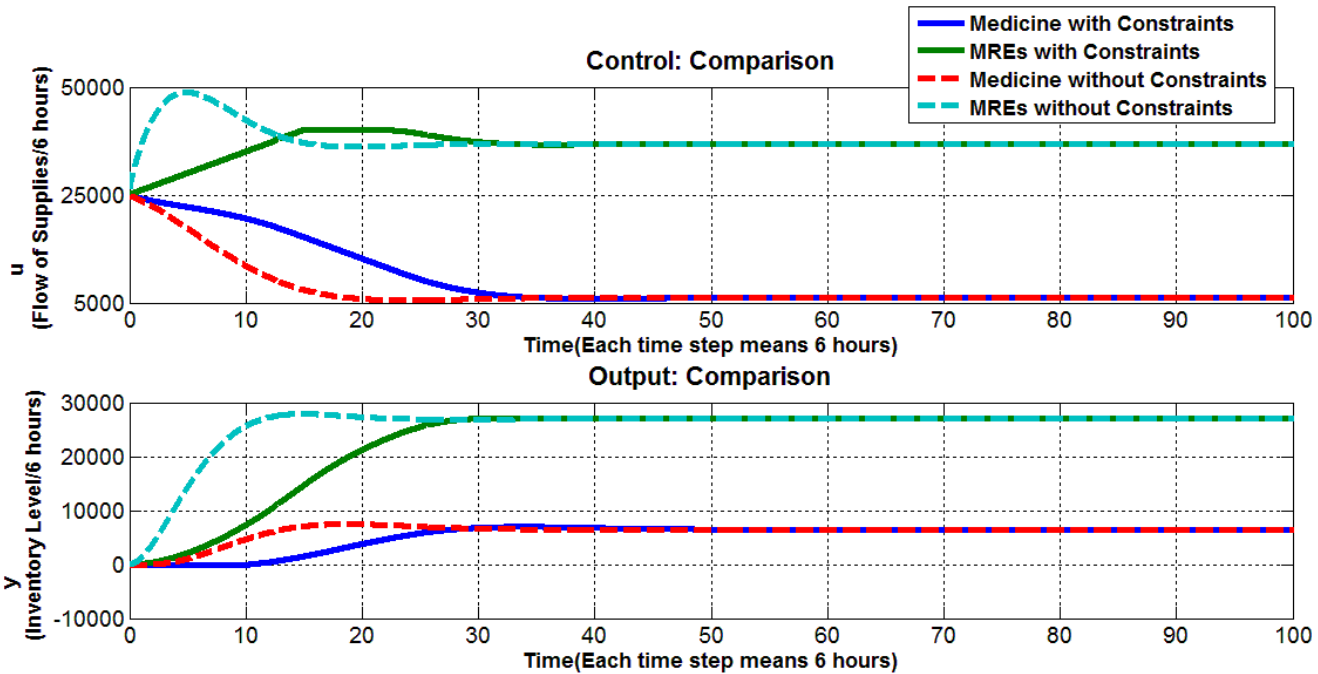

Derivative Control: Comparison

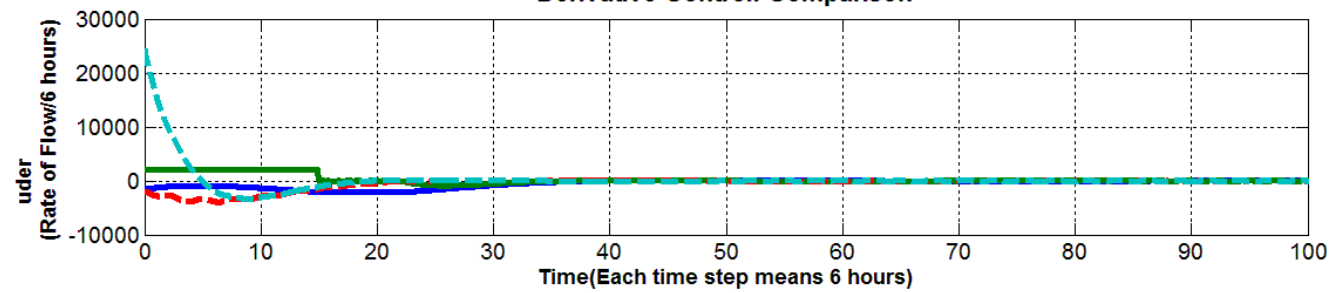

(b)

Figure 5.6 Online Continuous Time Model Predictive Control Results for the Base Case: Results with and without Constraints (Figure 5.6.a) and Comparative Results (Figure 5.6.b) 


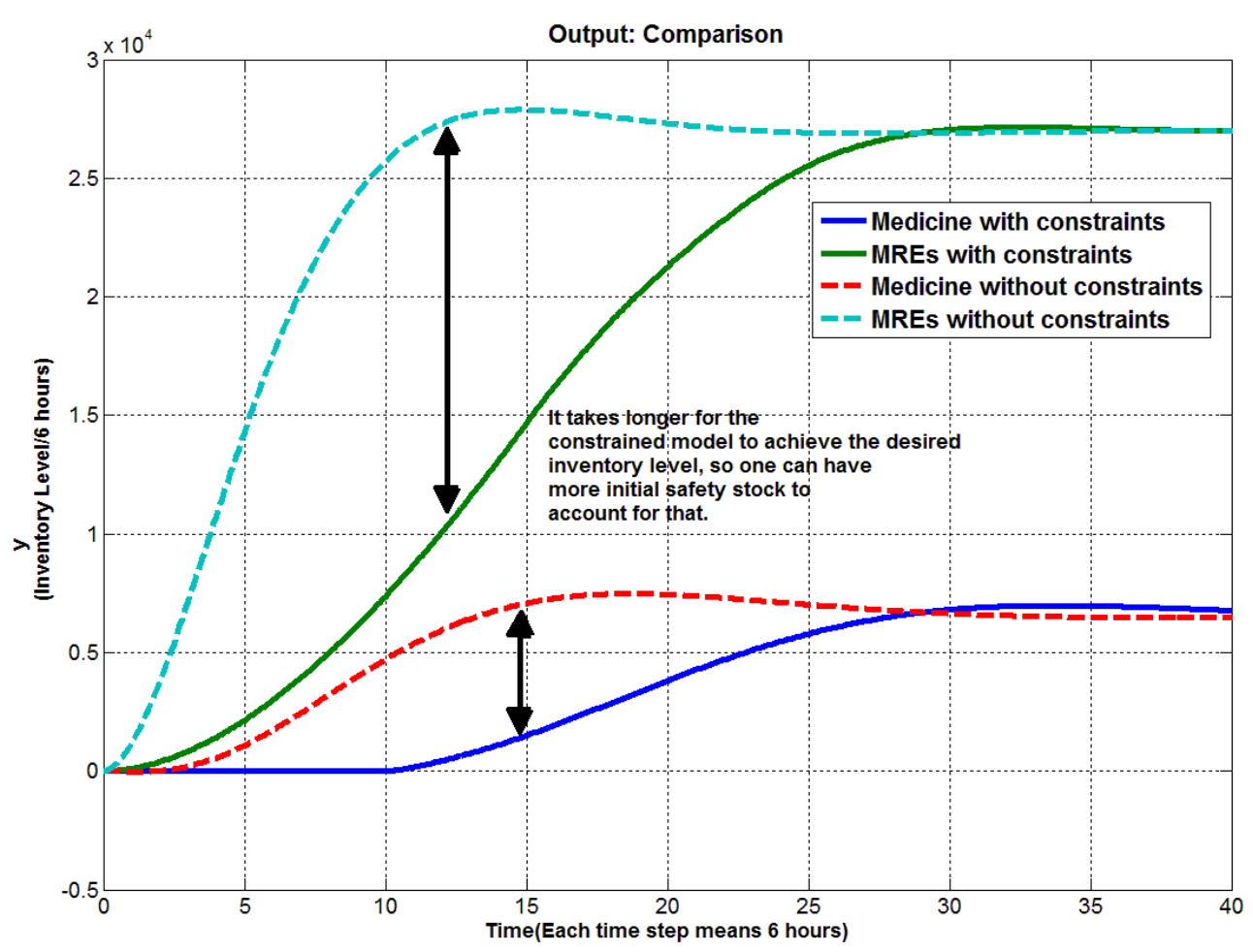

Figure 5.7 Online Continuous Time Model Predictive Control Results for the Base Case: Output Comparison for the Initial 10 Days

Next, only focusing on the case with constraints, we add white Gaussian noise into the system and gradually increase it in our emergency inventory control model using the following signal-to-noise ratio concept. The signal-to-noise ratio (SNR) is defined as follows:

$$
S N R=\left(\frac{R M S_{\text {Signal }}}{R M S_{\text {Noise }}}\right)^{2}
$$

We run the analysis with two SNR values, 30 and 40, to observe the response and investigate the performance of the model to obtain a stable trajectory. The results can be seen in Figure 5.8, where we increase the time period to 100 days (400 time steps) to be able to observe the effect of the noise more clearly. Figure 5.8 clearly indicates that the 
output inventory levels are still acceptable in such an unstable and unpredictable disaster environment, although the stochastic noise does affect the closed-loop responses, particularly the control and the derivative of the control signals. Since noise is introduced into the system to represent the stochasticity involved in the emergency inventory management operations, the model should be robust enough to respond to these stochasticities by efficiently converging to near-optimal target stock values, or at least staying within an acceptable range of optimality.

As observed from the Mozambique example, limitations on the rate of change on the flow of supplies in the emergency management framework requires extra attention. Intuitively, disruptions and the uncertainties within the emergency relief system can cause sudden rate changes in the supply flow and that rate change will gradually affect the delivery operations (e.g., a sudden increase due to a recently re-opened roadway, or a sudden decrease to unavailability of emergency vehicles). The values (positive or negative) for the limitations should be selected carefully by planners and emergency officials as that choice will directly influence the supply flow changes and therefore output inventory levels. By controlling this rate, the planner or emergency official can make decisions on increasing and decreasing the deliveries to a shelter where the number of people located in that shelter change rapidly in the aftermath of a disaster. 
Comparison with and without Noise
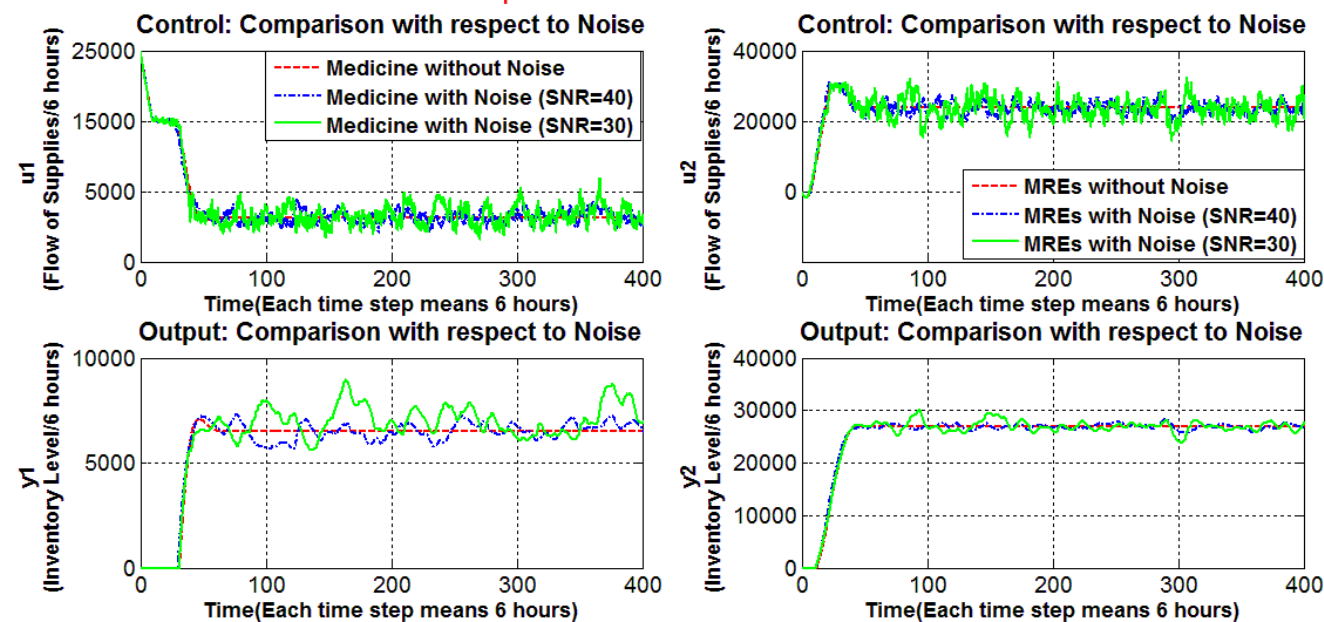

Derivative Control: Comparison with respect to Noise Derivative Control: Comparison with respect to Noise
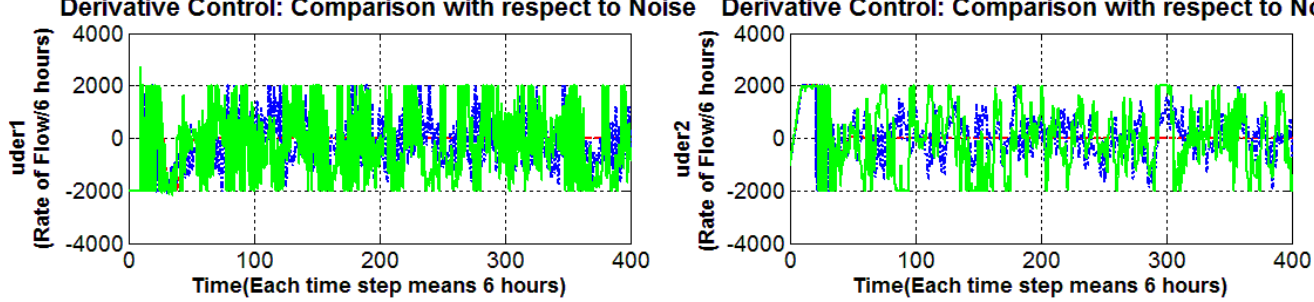

Figure 5.8 Online Continuous Time Model Predictive Control Results for the Base Case: Comparison with and without Noise

\subsubsection{Case 2: Increase in the Severity of the Disaster}

Using the demand increase results shown in Table 4.7, we would like to observe how our online model predictive model will perform with respect to the strength increase of Hurricane Katrina. For this case, the model becomes as follows: 


$$
\begin{gathered}
J=\eta^{T} H \eta+2 \eta^{T} F\left[\begin{array}{ll}
x_{1} & x_{2}
\end{array}\right]+\left[\begin{array}{ll}
x_{1} & x_{2}
\end{array}\right]^{T} \int_{0}^{T_{p}} e^{A^{T} \tau} Q e^{A \tau} d \tau\left[\begin{array}{ll}
x_{1} & x_{2}
\end{array}\right] \\
\text { subject to } \\
0 \leq y_{1} \leq 50000 \text { (Capacity in terms of units of medicine) } \\
0 \leq y_{2} \leq 50000 \text { (Capacity in terms of units of MRE's) } \\
10000 \leq u_{1} \leq 70000 \text { (Limitations on the flow of medicine, units/6 hr) } \\
10000 \leq u_{1} \leq 30000 \text { (Limitations on the flow of MRE's, units } / 6 \mathrm{hr} \text { ) } \\
-1000 \leq \dot{u}_{1} \leq 1000 \text { (Limitations on the rate of the medicine flow) } \\
-1000 \leq \dot{u}_{2} \leq 1000 \text { (Limitations on the rate of the MRE flow) }
\end{gathered}
$$

where the formulation of the matrices within the objective function and the state space formulation do not change from the base case. The weight matrices are again selected as $Q=C^{T} C=\left[\begin{array}{ll}1 & 0 \\ 0 & 1\end{array}\right]^{T}\left[\begin{array}{ll}1 & 0 \\ 0 & 1\end{array}\right], \quad R=0.1 I, \quad p_{1}=p_{2}=0.5, \quad T_{p_{1}}=10 / p_{1}, \quad T_{p_{2}}=10 / p_{2}, \quad$ and $N_{1}=N_{2}=3$. We use the three set point signals (target inventory values) from Table 4.7 (for demand $u=1,3,5$ ) so that $r_{\text {Medicine }_{1}}=6750, r_{\text {Medicine }_{2}}=7500$, and $r_{\text {Medicine }_{3}}=8250$ for the medicine, and two set point signals from Table 4.7 (for demand $u=1,5$ ) so that $r_{M R E_{1}}=30000$, and $r_{M R E_{2}}=42000$ for MRE's. That is, the target inventory level for the medicine is 6,750 at first, but then increases to 7,500 and then to 8,250 units with respect to the demand increase at the shelter for medicine within the time frame. MRE's, similarly, have an initial target inventory level of 30,000, which increases to 42,000 after an urgent need for more MRE's at the shelter. We obtain the coefficients of variation using the consumption and delivery distributions calculated as $\frac{\beta_{1}}{\alpha_{1}}=0.339,0.318,0.312$ and $\frac{\beta_{2}}{\alpha_{2}}=0.241,0.232$ from Table 4.7 via the offline planning model SHIC. This 
analysis is very significant, as it considers the possibility of an increase in consumption levels within the predicted future trajectory. Suppose the decision maker plans for the upcoming disaster using the offline planning model, SHIC, but during emergency relief operations, the demand levels change substantially in shelters. This will require an immediate action from the decision maker, who has the ability to track the flow of vital supplies in real time using the proposed online framework and to adjust the flow of emergency supplies accordingly. The result will be an adjustment in the safety stock levels to account for the needs of the evacuees located at shelters. This type of analysis can be used for slow onset disasters where the effects of the disaster cover a longer time period than in sudden disasters, for which the first case study analysis can be employed.

The results can be found in Figure 5.9 for more than 60 days (250 time steps) in the immediate aftermath of the hurricane where the stochasticity, which is highly possible within the emergency relief operations, has not been introduced yet in terms of noise. Similar to the base case, two types of results are presented to observe the differences between the unconstrained and constrained conditions. The results indicate that, in the Category 5 hurricane case, it takes much longer than in the base case for the derivative of the control, control, and therefore output to converge to optimal values. Although it takes longer, the model efficiently responds to the sudden changes in the demand levels of the victims and reaches the target inventory levels. The constrained case, in particular, where disruptions in the relief system affect the flow of vital supplies to shelters, makes it harder for our model to achieve a comfortable convergence process. Note that in the disaster relief period, there may not be enough commodities that can be distributed to a shelter from the usual supplier, or deliveries may not be possible due to a damaged 
transportation network. Therefore, any time delay caused by system constraints should be seriously taken into account for the sake of the emergency operations.

This fact leads to Figure 5.10, which shows a more focused picture of the behavior of the model in terms of outputs converging to optimal inventory levels. It is clear that the constraints on the flow of vital supplies due to disaster conditions significantly decelerate this convergence process. These constraints were all present during the recent disasters such as Hurricane Katrina or the earthquakes in Haiti and Japan, which clearly depicts the need of carefully studying the response with these constraints. This approach indicates the importance of safety stocks when emergency system constraints are present based on the flow of supplies and the capacity of emergency inventories. During this time period, emergency management officials can use the initial safety stock calculated as a buffer during offline planning instead of the vital supplies urgently needed. The model also reacts accordingly when there is an increase in the demand for the emergency supplies within the predicted horizon by increasing the inventory levels in a very short period of time. Therefore, this approach presents the opportunity to successfully respond to the fluctuating needs of disaster victims by making use of both offline planning and real-time feedback methodology results. 


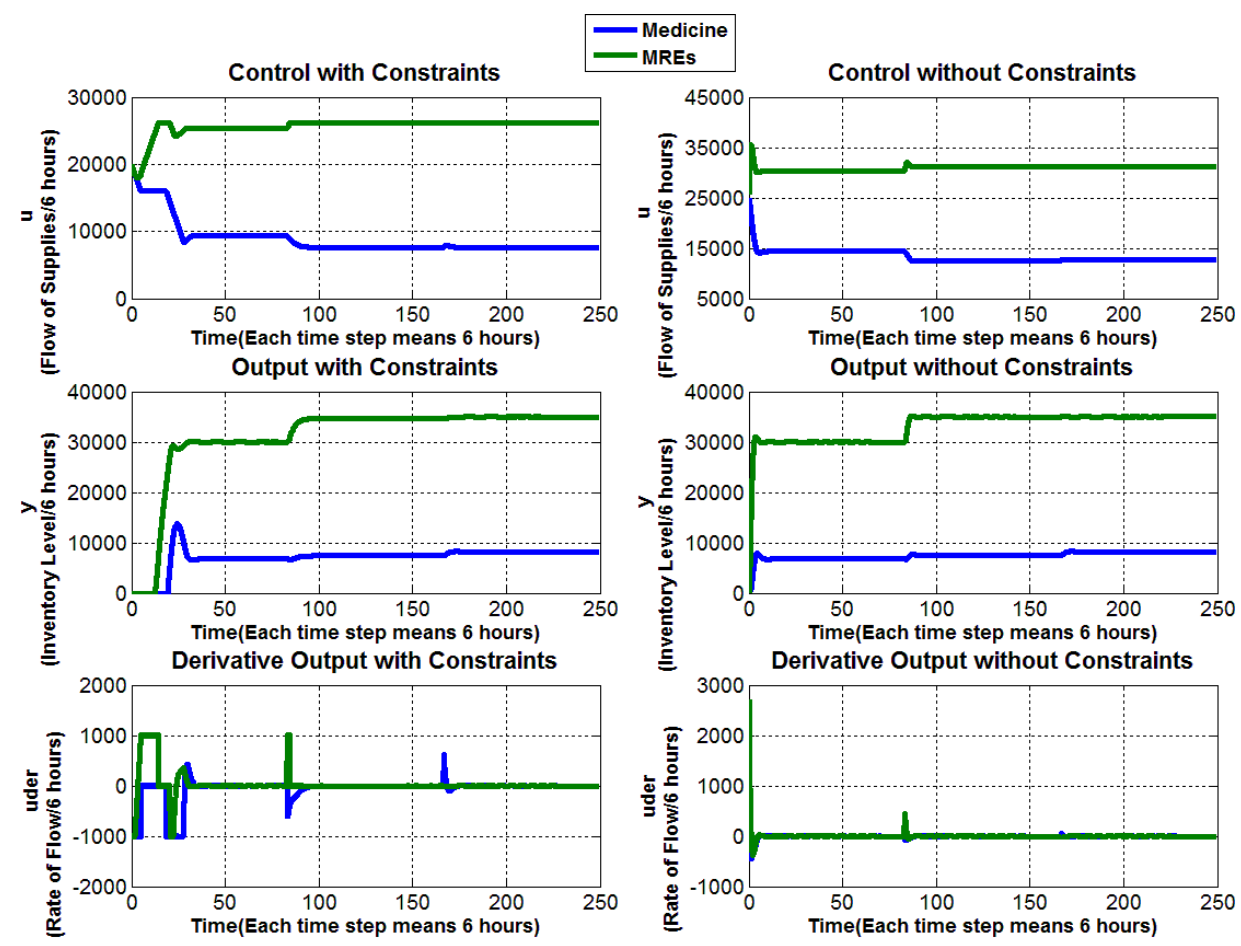

(a)
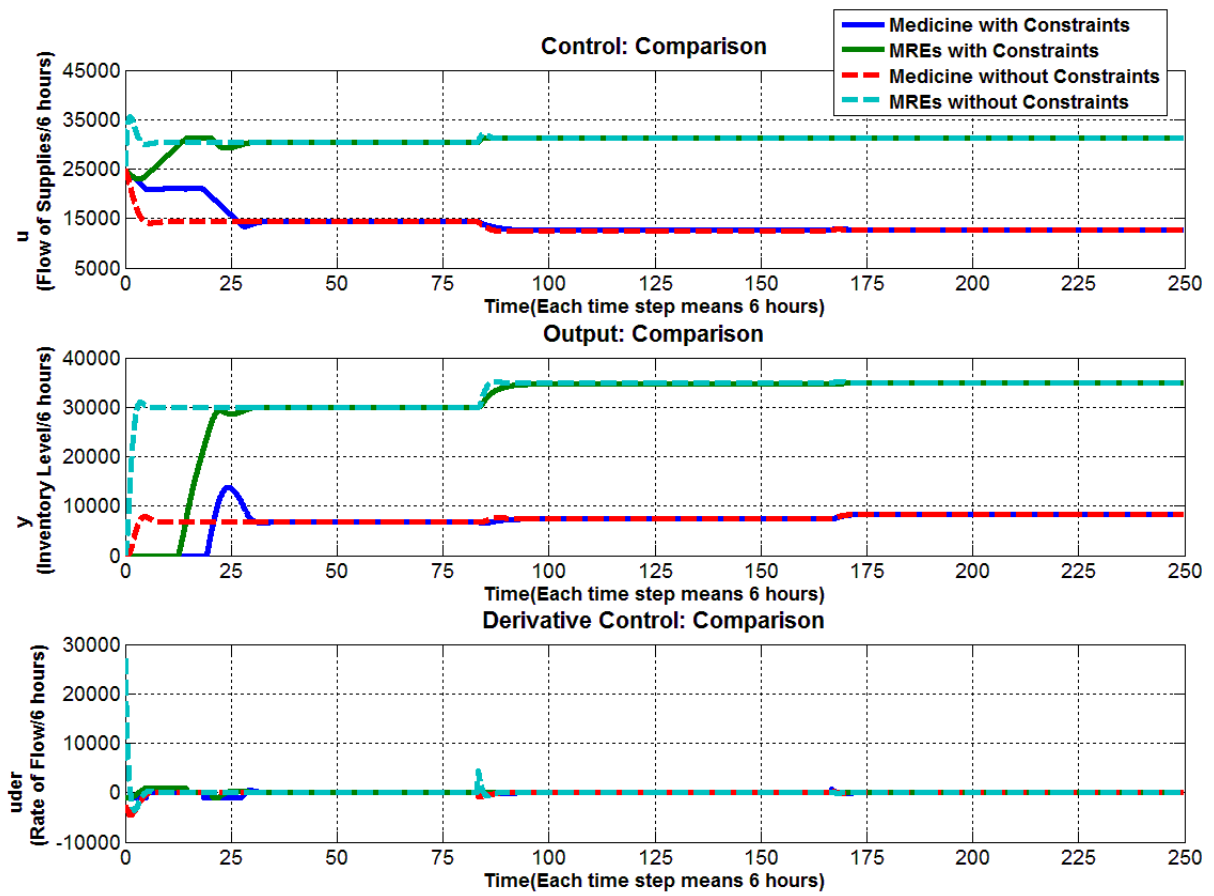

(b)

Figure 5.9 Online Continuous Time Model Predictive Control Results for the Case 2: Results with and without Constraints (Figure 5.9.a) and Comparative Results (Figure 5.9.b) 


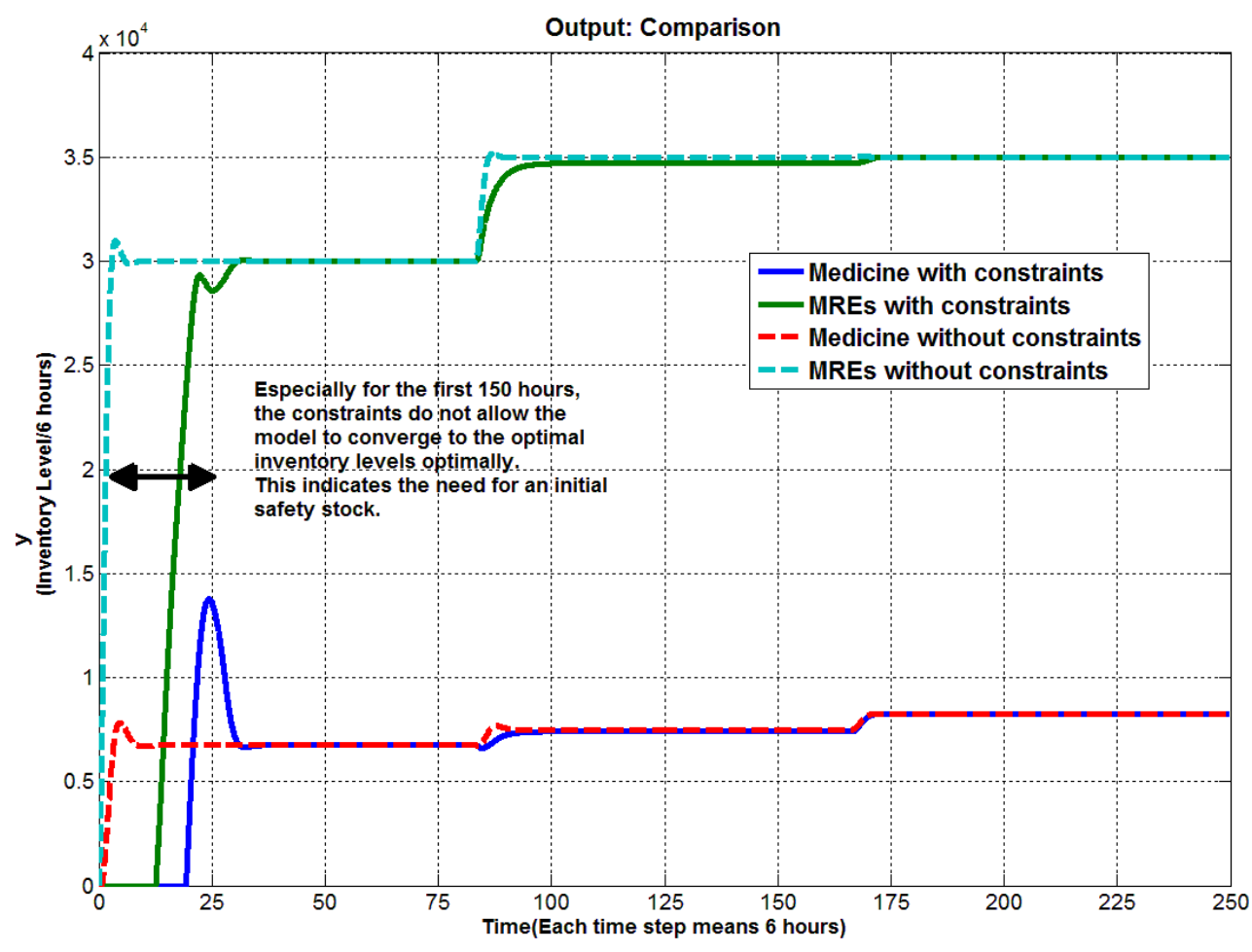

Figure 5.10 Online Continuous Time Model Predictive Control Results for the Case 2: Output Comparison

Next, only focusing on the case with constraints, we add the white Gaussian noise into the system (to the control, not the output) and gradually increase it in our emergency inventory control model using the signal-to-noise ratio concept. We ran the analysis with two SNR values, 30 and 40 again, to observe the response and investigate the performance of the model to obtain a stable trajectory for the vital supplies over time. The results can be seen in Figure 5.11. It is observed that the output inventory levels are deviating within $10 \%$ of the target inventory levels given the stochastic noise, which is introduced into the system to better simulate the extreme complexity expected under realworld emergency conditions. 
Comparison with and without Noise
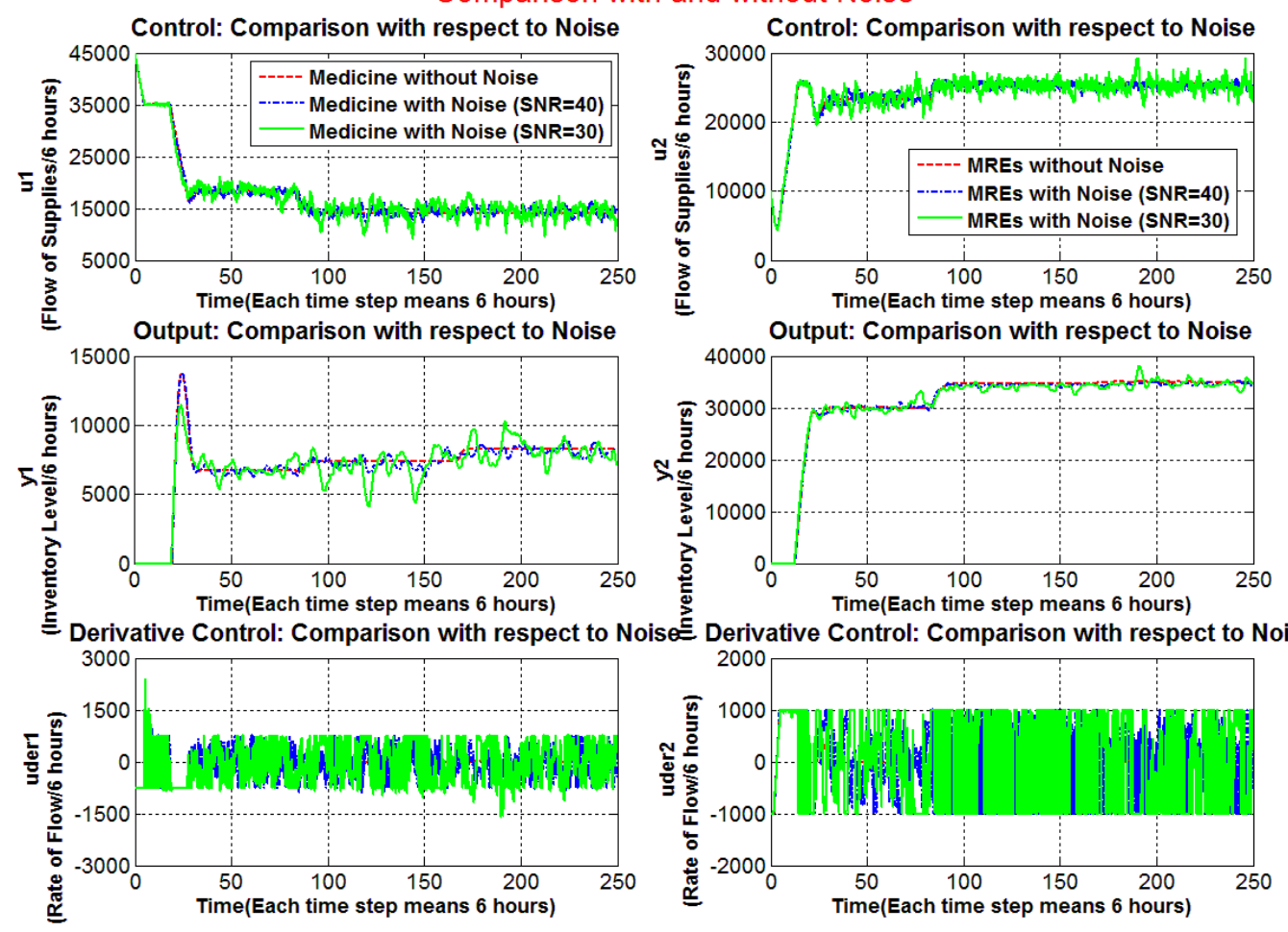

ise Derivative Control: Comparison with respect to Noise

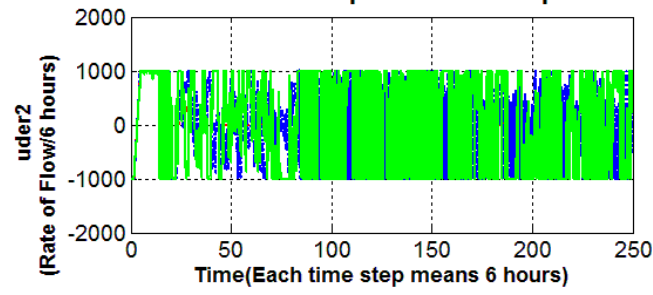

Figure 5.11 Online Continuous Time Model Predictive Control Results for the Case 2: Comparison with and without Noise

\subsubsection{Discussion}

The continuous time model predictive control methodology (CMPC) similarly requires the calculation of optimal target inventory levels via the multi-commodity SHIC model, as in the case of the functional approximation presented in the previous section. The second and more important purpose of SHIC within the model predictive control methodology is to determine and calibrate the model parameters given in Equation (5.64). The equation parameters $\alpha$ and $\beta$ are calculated via the consumption and supply distributions obtained using the multi-commodity SHIC model. Using these target values and model parameters, we solve the model predictive control problem given in Equation 
(5.52) to observe the real-time behavior of safety stock levels for vital supplies in the presence of disruptions within the emergency relief system.

The results of the continuous time model predictive online management study suggest that the proposed model can significantly improve emergency inventory operations in the aftermath of a disaster. Especially for dispersed and/or sudden onset disasters that spread over a large area, the realization of the increase in the number of deliveries may not be possible initially due to lack of trucks, personnel, and the commodity itself, or due to transportation-related problems such as the possible loss of vital connectors or links. On the other hand, the number of deliveries for slow onset disasters such as famine and drought may be more critical, as these rare disasters cause more losses and affect the lives of people more than others. Here, the critical point is to be able to adequately plan and prepare for the worst-case scenarios, where deliveries may not occur as they should, and therefore to have an initial safety stock that will prevent possible disruption at shelters in terms of insufficient vital supplies.

Another important point is that the proposed model is flexible enough to take into account the disruptions within emergency relief operations in terms of system constraints. In our model, these constraints include limitations on the safety stock levels of emergency supplies, regulating constraints on the flow of vital supplies to the emergency inventories, and rate constraints that limit the rate of change of this supply flow. Such constraints during emergency relief operations are very likely to exist due to demand fluctuations in the shelters, damaged transportation networks, and limited supply and deliveries, and they should be seriously taken into account. 
The model can respond to unexpected demand changes for vital supplies that can frequently happen in the aftermath of a disaster. The real-time response is an immediate increase in the flow of supplies and therefore inventory levels to satisfy the new demand. For this purpose, the proposed online predictive control model is fed with different target levels obtained from the SHIC analysis within the time frame in order to observe the behavior of the model while reaching new targets. With this approach, the decision maker has the ability to track the flow of vital supplies in real time using the proposed online framework and to adjust the flow of emergency supplies accordingly.

Random disturbances are also introduced into the emergency management system as stochastic noise to better simulate the extreme complexity expected under real-world emergency conditions. In particular, for the worst-case scenario, where the constraints and disruptions in the relief system affect the flow of vital supplies to people located in shelters, it takes longer for the model to achieve a comfortable convergence to the optimal inventory levels.

\subsection{RFID Integration}

Effective emergency inventory management with the proposed framework depends upon consolidating, integrating and analyzing data collected from sources such as emergency inventories and shelters. Conventional tracking systems require manual intervention, which may be labor intensive, time consuming and error-prone. RFID systems, on the other hand, give a total visibility of commodity movement in the disaster

supply chain. By feeding this data into the SHIC model in terms of the amounts of consumption and deliveries at the shelters, one can make early decisions about pre- 
stocking in case there is a possibility of serious interruptions in the supply. Use of these accurate distributions within the SHIC model will result in obtaining accurate safety stocks, and therefore achieving efficient planning for disaster inventories. This planning information can be used to feed the proposed online methodologies to be able to facilitate accurate real-time inventory level tracking.

\subsubsection{Proposed Setup}

To be able to make use of RFID systems within the emergency inventory management framework, RFID devices should be deployed in the emergency inventories, distribution centers and shelters (see Figure 3.8). If possible, in the inventories and distribution centers, pallets should be tagged and each location in the inventory racks should be tagged with a location identifier. Forklifts should be equipped with RFID readers so that as each pallet is put away, the pallet ID with respect to the specific commodities carried can be captured. Similarly, in the shelters, RFID readers can be located at the shelter entrance to capture the tag information when emergency supplies are being delivered. Efficient design and construction of RFID systems is significant to be able to make RFID-based tracking possible.

One of the important issues while designing the RFID systems is the decision on which tag type to use. There are two options for that purpose: active or passive tags (see Section 3.1 for more details). Since active tags have batteries in them, they have certain advantages over passive tags for the disaster relief operations. Their signal is stronger, and their signal can be received without activating the reader, which will be very helpful as it may not be possible for emergency officials to be constantly focused on tracking and 
reading the tags. However, the cost of active tags is higher than passive tags. On the other hand, passive tags have a much higher lifespan, lasting indefinitely whereas active tag batteries should be replaced typically within 2-3 years. Based on this information and given the specific problems of a disaster such as unavailability of personnel and monetary problems, selection of the tag type should be carefully examined. Another key system design is to decide on the type of antenna (circular or linear) that should be deployed with the RFID reader. A linear antenna is able to read from longer distances whereas a circular one can read from a much wider angle. Depending on the specific disaster conditions, it is possible to choose the type of antenna most useful for tracking purposes.

\subsubsection{Advantages of the RFID Based Tracking Approach}

RFID devices have three major advantages for the proposed inventory management framework in order to ensure efficient and reliable inventory management and planning operations:

- Tracking: RFID has the online tracking capability that works to identify the motion of any emergency supply. The idea includes not only real-time position tracking (flow of commodities), but also tracking motion through entry and exit points. This online tracking will provide emergency officials with supply flow data that can be used to obtain commodity consumption and demand distributions. These distributions are critical inputs to the proposed emergency inventory framework. 
- Identification, sensing, and authentication: This can be used for identifying the victims of the disaster to have accurate information. Most importantly, this will help to accurately respond to the needs of patients in the disaster area. Within the proposed framework, this information can be used during the process of tracking vital/perishable commodities such as medicine and blood to enable secure medical assistance to victims. For this case, RFID data for valuable and perishable goods such as, medicine and blood, can be given as inputs to the SHIC model in order to conduct a single-commodity analysis given in subsection 4.4.3.5.

- Automatic data collection and transfer: This feature of RFID systems helps to minimize data entry and collection errors and reduces information processing time and inventory management problems. With the minimal data processing error, it is possible to obtain more accurate distributions and feed them into the SHIC model so that the emergency inventory management framework can respond to the needs of the victims located in the shelters efficiently.

\subsubsection{Limitations and Disadvantages}

First of all, design of RFID systems within the emergency inventories, distribution centers and shelters require a significant effort with the incorporation of federal/state agencies and private sector companies. Regarding the design issues, there are several disadvantages that exist for the use of RFID devices within our integrated framework (see Section 3.1 for more details): 
- RFID systems are often more expensive than conventional barcode systems. However, cost and implementation problems can be handled by a cost-justified incorporation of federal/state agencies as well as private companies such as RFID vendors.

- Radio frequency (RF) gives RFID devices its strength, but also creates the weaknesses in terms of communication. RF carries the information between the reader and tag. If the communication infrastructure is down or if there is not enough RF signal strength, RFID systems will not be able to work to feed data into the SHIC model.

- RFID systems will successfully work only if three components work properly: tag (contains ID, or serial number), reader (reads the information from the tag), and computer (gets the information from the reader and stores it). As long as there is an emergency official who can use and interpret the data from RFID tracking to obtain the flow distribution, the proposed emergency inventory management framework can be used for planning and online tracking purposes efficiently.

\subsection{Summary of Key Findings}

In order to improve the efficiency and security of disaster supply chains, traceability and visibility of the flow of supplies are of critical importance. Existing models reviewed in Chapter 2 show good efforts towards planning purposes but also show the limitations, such as the lack of online tracking, and not being robust to real-life disruptions and limitations. This chapter presents a RFID-based solution for real-time 
tracking of disaster inventories and supply flows integrated with the planning model SHIC. Use of RFID data to feed the SHIC model results in obtaining accurate safety stocks, and therefore achieving efficient planning for disaster inventories. This planning information can then be used to feed the proposed online methodologies and facilitate real-time inventory level tracking and to allow flexible demand and supply processes as well as securing buffer inventories as safety stocks.

Intuitively, the first proposed model, namely functional approximation, focuses on bringing the system back to optimality in real-time when the actual inventory levels deviate from the optimal (target) ones. Two approaches are used for this purpose: neural networks and the SPSA algorithm. The neural network-based approach shows better performance in terms of time; however, the SPSA algorithm clearly achieves to inventory levels optimally closer to target values obtained from the planning model SHIC. Therefore, the planner or decision maker should be careful to choose any of these two algorithms based on the specific disaster conditions on hand. If time is a major concern as in the case of sudden onset disasters, it may be logical to use neural networks. On the other hand, for slow onset disasters, where calculation of optimal inventory levels more accurately may be more important than time, SPSA can be efficiently used to obtain the emergency inventory levels.

As aforementioned, these two models have limitations on mathematical convergence, stability and they do lack real-life constraints. The CMPC model, on the other hand, solves these problems and offers an efficient method for online tracking of disaster inventories by emergency officials. The CMPC model not only considers the uncertainty in disaster operations as adding random noise to the system, but it also 
considers the constraints that are present in real-life disaster operations. Here, it is important to note that consideration of real-life constraints has a negative effect on response time. That is, real-life constraints on inventory levels and supply flow increase response time. Therefore, if one has limitations on inventory levels and the flow of supplies, it takes longer to achieve the desired inventory levels as stocks and deliveries are limited. Emergency officials can efficiently use the proposed methodology to analyze the specific disaster conditions and decide on the additional safety stocks that will be used to accommodate for the long response time.

As expected, presence of noise in the system creates output inventory levels that diverge from the optimal ones. However, even with the uncertainty introduced, the proposed CMPC model behaves reasonable enough as the outputs are still within a $10 \%$ range of the optimal levels. Using this methodology, planners can determine the initial safety stock levels based on this analysis and account for the lost inventories due to the divergence from the optimal inventory stocks during the disaster relief period.

To sum up, as clearly stated by Whybark (2007), the management of the physical commodities in storage can be improved if there are more effective tracking and monitoring systems. Functional approximation and CMPC techniques, therefore, can be used efficiently for real-time tracking of emergency inventory levels and the flow of supplies given the data obtained from the SHIC model based on accurate RFID consumption and delivery distributions. 


\section{CHAPTER 6. CONCLUSIONS AND FUTURE RESEARCH}

This thesis proposes a novel RFID-based online emergency management framework that can be effectively used to synchronize critical delivery and consumption processes, to facilitate sustainable resource tracking, and to provide an efficient distribution of flow in support of disaster relief efforts. The objective of this thesis is twofold. First, a framework is presented to understand the critical components and requirements of the proposed emergency inventory management methodology, mainly focusing on RFID-related issues. Second, an efficient mathematical model is developed utilizing both an offline inventory planning strategy and online control theoretical methodology that can be integrated with the help of RFID technologies as the main resource-tracking tool. Given the time-variant demand for emergency supplies during emergency relief operations, the proposed methodology can be used efficiently for the dynamic stocking of vital supplies and online management of the flow of these stocks to emergency shelters. Moreover, representing the inventory management component of the overall emergency management system given in Figure 1.2, our model can serve as an efficient and flexible tool within the emergency disaster plans or scenarios created by state and federal agencies.

The literature on emergency inventory management studies clearly indicates the need for a robust integrated emergency management framework that consists of a planning model and an online strategy to make sure that the planned strategy for the flow of emergency supplies continues without any disruption in the aftermath of a disaster. 
Therefore, the novelty of this thesis is that, for the first time in the emergency inventory management field, the proposed approach combines an offline planning strategy with online control techniques in a single framework that is robust with respect to disruptions in the supply and consumption of vital commodities in the aftermath of a disaster. Within this framework, the offline planning problem is solved by the stochastic humanitarian inventory management approach, whereas the online modeling strategies include the application of neural network-based functional approximation, simultaneous perturbation stochastic approximation (SPSA), and continuous time model predictive control (CMPC) techniques. This integrated real-time inventory management system not only allows the efficient usage and distribution of emergency supplies, but also offers the ability to be integrated with emerging ITS technologies such as Radio Frequency Identification Devices (RFID) for online commodity tracking and logistics.

The offline planning component of our proposed framework is the stochastic humanitarian inventory control (SHIC) model first proposed by Ozbay and Ozguven (2007). This model attempts to determine the minimum safety stock levels in emergency inventories so that the consumption of these stocked commodities at major shelters can occur with minimum disruption. The results of the model, when applied to various case studies, are encouraging in the sense that they provide a basis for the realistic analysis of the risk involved in planning for safety stocks, whereas the flexibility of the model allows the application of the mathematical formulation to any extreme event. The proposed casebased approach can easily be incorporated into disaster relief plans so that planners can become aware of potentially serious inventory-related problems that can occur at shelters. 
Several realistic case studies built using information available from past disasters are also carefully studied using the model, addressing the following important issues:

- Change in the severity of the disaster,

- Disruptions in the transportation network,

- Emergency orders/cross shipping possibilities,

- Change in the consumption and delivery distributions, and skewness.

The findings of the offline planning analysis can be listed as follows:

- An increase in the strength of the disaster leads to an increase in the demand for vital supplies at shelters. Therefore, as the consumption of a given commodity increases, the corresponding initial safety stock values also increase.

- The additional safety stock required decreases as the number of deliveries during the emergency relief period increases. Thus, if the procurement and infrastructure systems are robust enough to support a higher level of deliveries, the authorities can afford to maintain lower levels of safety stocks. This will reduce the cost of emergency preparedness without compromising the wellness of evacuees.

- The transportation of vital and perishable commodities and the use of different suppliers for distribution also require extra attention when determining optimal safety stock levels.

- The risk and uncertainty involved during emergency relief operations directly influence the consumption and delivery distributions that may have high mean or variance, or may be highly skewed. Therefore, different consumption and delivery 
patterns and high skewness should be seriously considered when conducting a realistic emergency inventory management analysis.

The proposed SHIC model is also used as the offline base for our overall emergency inventory management framework in order to:

- determine the optimal inventory levels of the proposed online feedback control methodologies,

- simulate the data (including the stochastic consumption and deliveries of vital commodities) needed by the function approximator, and

- calculate the model parameters for the continuous time model predictive controller.

As the first online control application, the function approximator (FA) and the SPSA algorithm are used to solve the unconstrained nonlinear minimization problem shown in Equation (5.4) to bring the system back to optimality in real time when the actual inventory levels deviate from the optimal (target) ones. For the function approximation, a neural network-based approach is proposed. When disruptions or fluctuations occur in the system, we invoke the neural network, and the underlying parameters are estimated by training to bring the inventory levels back to optimal target inventory levels. This is actually a feedback control methodology that does not require any information about the equations or models governing the stochastic disaster relief system. It adjusts the system parameters using a closed-loop control approach rather than an open-loop model used by the conventional optimal control approaches available in the literature (Zhang and Ritchie, 1997; Shen and Kong, 2009; Peng et al., 2011). The SPSA 
algorithm, similarly, is used to estimate the descent direction for the unconstrained minimization problem of Equation (5.4) in the presence of noisy measurements and fluctuating target inventory levels. The experiments have been conducted to compare the performance of the online control methodology with the two stochastic approximation algorithms, namely the function approximator with Levenberg-Marquardt (LM), and SPSA. The algorithms are applied to optimally determine the weights of the neural network at each iteration with different amounts of noise associated with stochastic consumption and deliveries. The performance comparison of the two algorithms is based on the analysis of the objective functions, the optimal inventory levels, and the overall computational times. As a result of the comparative analysis, it is observed that overall computational times are always better for the LM algorithm. However, the results indicate that LM does not always converge to the optimal target inventory values in the presence of stochasticity, whereas the SPSA algorithm definitely has better performance than LM in terms of convergence. This is due to employing a nominal state during the SPSA procedure, which allows us to find better inventory level value results at the end of the runs, in addition to increasing the computational times. Although computational time is also a significant concern to reach optimality for disaster relief operations, SPSA still performs well and remains within a range of 20 to 40 seconds while converging more efficiently to the target levels of vital supplies.

However, these methods have several crucial disadvantages, as follows:

- Models do not provide a means of analytically computing the real-time trajectory of the flow of vital supplies and emergency inventory levels. 
- Convergence to optimal inventory values is not necessarily satisfied and directly depends on the selection and performance of the system parameters.

- This dependence on the parameters creates the problem of instability for our emergency relief system, which should be avoided.

- Constraints in terms of inventory capacity and limitations on the delivery of supplies that are possibly encountered in real-world disaster systems cannot be solved with these methods.

Given these limitations of the neural network- and SPSA-based models, we propose a second control methodology that includes a more efficient approach based on the continuous time model predictive control model, and that can address the need for mathematical convergence, stability, tracking, and constrained minimization. This model introduces the ability to analytically compute the trajectory of the emergency inventory levels and the flow of vital supplies in contrast to the functional approximator method. Therefore, it gives the planner/decision maker the opportunity to keep track of demand fluctuations for vital supplies (food, water, medical supplies, etc.), and to be prepared for transportation network disruptions during the disaster relief period. The core component of this methodology is predicting the inventory levels of emergency supplies for a future horizon given the information at the current time, and a cost function to be minimized based on the difference between the desired (obtained via the SHIC model) and the actual responses. With this idea, the quadratic programming problem given in Equation (5.52) is solved with and without constraints on inventory levels, the flow of supplies, and the rate 
of change of these flows. Moreover, the uncertainty within emergency relief operations due to unexpected fluctuations in the consumption and delivery of vital supplies is introduced into the system as white Gaussian noise.

The results of the analysis suggest that the proposed framework can handle the unexpected fluctuations likely to happen during emergency relief operations. The constrained conditions that can occur due to the fact that emergency vehicles may not be able to supply shelters, or that some inventories may be lost due to disaster effects, can also be studied extensively with the CMPC model. Therefore, planners and decision makers can be aware of the time delay due to disaster-related disruptions and can maintain a safety buffer for vital supplies. During the relief period, emergency management officials can account for the insufficiency of vital supplies detected in real time by the CMPC model using the initial safety stock that was calculated as a buffer during the offline planning process. This indicates the importance of utilizing both offline planning and real-time feedback methodology results in order to successfully respond to the needs of disaster victims. Furthermore, given the constraints of the emergency relief system and the high levels of stochasticities in the flow of emergency supplies, the proposed model, for the worst case, deviates at most $10 \%$ around the target inventory safety stock levels obtained via the offline planning model.

With these findings, the proposed integrated model can be used to examine the differences in inventory strategies for different types of disasters as follows: 
- Dispersed and sudden onset disasters: This type of disaster is the most difficult one in terms of emergency response, as it spreads over and therefore affects a large region and happens very fast, as in the case of the 2004 Indian tsunami. As a result, at the very beginning, the variances of consumption and delivery distributions will be very high; therefore, the planner should be aware of the consequences of not having optimal safety stocks in emergency inventories initially. Here, an analysis based on the changes in mean and variance from the second multi-commodity case study given in Section 4.3.3.2 (modeling different distributions) will be crucial. The strategy should be keeping more inventory safety stock initially to account for the high demand in the early aftermath of a disaster, which can be obtained by distributions with high variance and mean in Figure 4.8. The feedback control methodology proposed via the neural network and SPSA models can be employed here to obtain the target inventory stock levels and to respond to the disruptions in the system, as the models governing the stochastic disaster relief system will be the most difficult to obtain for this type of disaster. Although skewness can also be used as an alternative strategy, that concept is more useful for localized, sudden onset disasters where the initial demand becomes enormous and concentrated in a small region.

- Dispersed and slow onset disasters: This type of disaster also spreads over and affects a large region, but it covers a longer time period than sudden disasters. Examples include the 2009 avian flu epidemic and the famines in Africa. In this case, the variances and needs vary with time, where response will require thorough study of different consumption and delivery patterns. Analytically 
computing the real-time trajectory of the flow of vital supplies and emergency inventory levels with CMPC is crucial for this type of disaster. Based on the analysis results of the multi-commodity case study given in Section 4.3.3.3 (disruptions to the transportation network), it can be concluded that, for this type of disaster, there is not necessarily a need for a huge initial safety stock at the very beginning, but depending on the strength of the disaster and the number of deliveries that can be made to shelters, safety stocks for vital supplies should be adjusted in emergency inventories using the distributions in Figure 3 and the online tracking component presented in the previous section.

- Localized and slow onset disasters: This type of disaster affects a specific location rather than a large region, and it covers a long time period, as the severity of the disaster increases gradually. An example is the infamous 2005 Hurricane Katrina. As extensively studied in the multi-commodity case study of the SHIC analysis given in Section 4.3.3.1, this type requires the ability to respond to disaster disruptions, focusing on different consumption and delivery distributions and change in the severity of the disaster. As this type is localized and slow, emergency officials can be more efficient than in other types of disasters in terms of adjusting safety stocks. In order to make accurate adjustments for safety stocks, it is essential to be able to facilitate the online tracking mechanism to control the flow of vital supplies.

- Localized and sudden onset disasters: This type of disaster also affects a specific location rather than a large region, but it happens very quickly as in the case of the 2010 Haiti and 2011 Japan earthquakes, where the initial need for vital 
supplies was enormous. In this case, distributions for emergency supplies can be highly skewed, especially in the early periods of the disaster. The skewness-based analysis results of the multi-commodity case study given in Section 4.3.3.2 (modeling different distributions) show that, as an emergency inventory strategy for this case, it is appropriate to use this analysis to determine the initial inventory stocks given the distributions in Table 4.8 when there is a huge demand for emergency supplies at the beginning of the disaster, creating the need for a leftskewed distribution.

Based on this information, a summary of our model application for different types of disasters can be seen in Table 6.1. 
Table 6.1 Application of the Proposed Model for Different Types of Disasters

\begin{tabular}{|c|c|c|c|}
\hline \multirow{2}{*}{ 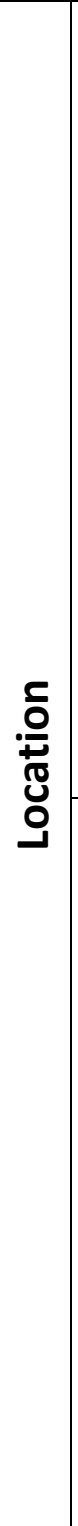 } & 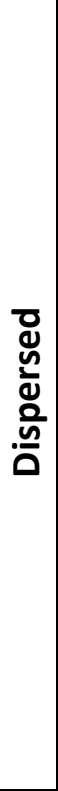 & $\begin{array}{c}\text { This type of disaster also spreads over } \\
\text { and affects a large region, but it covers } \\
\text { a longer time period than sudden } \\
\text { disasters. Examples include the } 2009 \\
\text { avian flu epidemic and the famines in } \\
\text { Africa. } \\
\text { Analytically computing the real-time } \\
\text { trajectory of the flow of vital supplies } \\
\text { and emergency inventory levels with } \\
\text { CMPC is crucial for this type of } \\
\text { disaster. }\end{array}$ & $\begin{array}{c}\text { This type of disaster is the most difficult } \\
\text { one in terms of emergency response, as it } \\
\text { spreads over and therefore affects a large } \\
\text { region and happens very fast, as in the case } \\
\text { of the } 2004 \text { Indian tsunami. } \\
\text { An analysis based on the changes in mean } \\
\text { and variance from the second multi- } \\
\text { commodity case study given in Section } \\
4.3 .3 .2 \text { (modeling different distributions) } \\
\text { will be crucial. } \\
\text { The feedback control methodology } \\
\text { proposed via the neural network and SPSA } \\
\text { models can be employed here to obtain the } \\
\text { target inventory stock levels and to respond } \\
\text { to the disruptions in the system }\end{array}$ \\
\hline & \multirow[t]{3}{*}{ 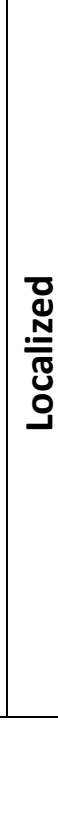 } & $\begin{array}{l}\text { This type of disaster affects a specific } \\
\text { location rather than a large region, and } \\
\text { it covers a long time period, as the } \\
\text { severity of the disaster increases } \\
\text { gradually. An example is the infamous } \\
2005 \text { Hurricane Katrina. } \\
\text { As extensively studied in the multi- } \\
\text { commodity case study of the SHIC } \\
\text { analysis given in Section } 4.3 .3 .1 \text {, this } \\
\text { type requires the ability to respond to } \\
\text { disaster disruptions, focusing on } \\
\text { different consumption and delivery } \\
\text { distributions and change in the } \\
\text { severity of the disaster. }\end{array}$ & $\begin{array}{l}\text { This type of disaster also affects a specific } \\
\text { location rather than a large region, but it } \\
\text { happens very quickly as in the case of the } \\
2010 \text { Haiti and } 2011 \text { Japan earthquakes, } \\
\text { where the initial need for vital supplies was } \\
\text { enormous. } \\
\text { In this case, distributions for emergency } \\
\text { supplies can be highly skewed, especially } \\
\text { in the early periods of the disaster. }\end{array}$ \\
\hline & & Slow & Sudden \\
\hline & & \multicolumn{2}{|r|}{ Time } \\
\hline
\end{tabular}

\subsection{Summary of Contributions}

Summary of all the contributions of this dissertation (given in detail in the previous section) can be seen in Table 6.2 where the corresponding information for each contribution is clearly presented. 
Table 6.2 Summary of Contributions

\begin{tabular}{|c|c|}
\hline Contribution & Information \\
\hline $\begin{array}{c}\text { Integration of Offline } \\
\text { Planning and Online } \\
\text { Control }\end{array}$ & $\begin{array}{c}\text { The proposed approach combines an offline planning strategy } \\
\text { with online control techniques in a single framework that is } \\
\text { robust with respect to disruptions in the supply and } \\
\text { consumption of vital commodities in the aftermath of a } \\
\text { disaster. }\end{array}$ \\
\hline RFID Integration & $\begin{array}{c}\text { This integrated real-time inventory management system offers } \\
\text { the ability to be integrated with emerging ITS technologies } \\
\text { such as Radio Frequency Identification Devices (RFID) for } \\
\text { online commodity tracking and logistics. }\end{array}$ \\
\hline $\begin{array}{c}\text { Online Tracking of } \\
\text { Disaster Inventory } \\
\text { Operations }\end{array}$ & $\begin{array}{c}\text { During the relief period, emergency management officials can } \\
\text { account for the shortage of vital supplies detected in real time } \\
\text { by the CMPC model using the initial safety stock calculated as } \\
\text { a buffer during the offline planning process. }\end{array}$ \\
\hline $\begin{array}{c}\text { Applicability to } \\
\text { Different Types of } \\
\text { Disasters }\end{array}$ & $\begin{array}{c}\text { By carefully selecting the parameters and conducting } \\
\text { sensitivity analyses on different scenarios, the proposed } \\
\text { framework can be successfully applied on different types of } \\
\text { disasters based on the duration of the impact and size of the } \\
\text { impact area. }\end{array}$ \\
\hline $\begin{array}{l}\text { Modeling } \\
\text { Uncertainty }\end{array}$ & $\begin{array}{l}\text { Uncertainty (stochasticity) involved in the disaster relief } \\
\text { operations (due to disruptions in the transportation network, } \\
\text { fluctuating demand and supplies, lost inventories, } \\
\text { unavailability of the personnel and vehicles, drastic changes in } \\
\text { the disaster strength, etc.) can be modeled in the framework by } \\
\text { carefully imposing the noise (error) into the system. }\end{array}$ \\
\hline $\begin{array}{l}\text { Modeling } \\
\text { Constraints }\end{array}$ & $\begin{array}{l}\text { The constrained conditions (on inventory levels, flow of } \\
\text { supplies and rate of change in that flow) that can occur due to } \\
\text { the fact that emergency vehicles may not be able to supply } \\
\text { shelters, or that some inventories may be lost due to disaster } \\
\text { effects, can be studied extensively. }\end{array}$ \\
\hline $\begin{array}{c}\text { Response to Real-Life } \\
\text { Cases }\end{array}$ & $\begin{array}{c}\text { The proposed model offers the ability to study distinct real-life } \\
\text { cases with analyzing the following issues: change in the } \\
\text { strength of a disaster, disruptions in the transportation network, } \\
\text { fluctuations in the supply and demand, vital/perishable } \\
\text { commodities, emergency/cross shipping possibilities, and } \\
\text { multi-suppliers. }\end{array}$ \\
\hline
\end{tabular}




\subsection{Key Findings}

The overall findings of this thesis (Table 6.3) have pointed out that the proposed integrated framework can be efficiently used as a state-of-art practice for emergency inventory planning and inventory control during relief operations without ignoring the real-world uncertainties, fluctuations, and constraints of disaster conditions.

Table 6.3 Summary of Key Findings

\begin{tabular}{|c|c|c|}
\hline Issue & Findings \\
\hline $\begin{array}{c}\text { Change in the } \\
\text { Severity of the } \\
\text { Disaster }\end{array}$ & $\begin{array}{c}\text { An increase in the strength of the disaster leads to an increase in the } \\
\text { demand for vital supplies at shelters. Therefore, as the consumption of } \\
\text { a given commodity increases, the corresponding initial safety stock } \\
\text { values also increase. }\end{array}$ \\
\hline $\begin{array}{c}\text { Drastic } \\
\text { Fluctuations in } \\
\text { the Demand } \\
\text { directly influence consumption and delivery distributions, which may } \\
\text { have high means or variance, or may be highly skewed. The propose } \\
\text { model calculates more initial safety stocks to account for the high } \\
\text { variances in consumption and delivery distribution of vital supplies. } \\
\text { The additional safety stock values tend to increase as the left skewness } \\
\text { of the commodity distribution increases to account for the huge } \\
\text { consumption demand coming from victims located at shelters at the } \\
\text { early stages of the disaster. Using this analysis, different consumption } \\
\text { and delivery patterns and high skewness can be taken into account } \\
\text { when performing a realistic emergency inventory management } \\
\text { analysis. }\end{array}$ \\
$\begin{array}{c}\text { As the disaster strength increases, the initial safety stock appears to be } \\
\text { insufficient, and a huge amount of additional safety stock is needed } \\
\text { when the number of deliveries is limited due to damaged/congested } \\
\text { roadways. This is because, if the system is highly stochastic, the } \\
\text { probabilistic constraints are satisfied only if additional safety stock is } \\
\text { high. This will impose higher costs. However, if the initial safety stock } \\
\text { is too low, the problem becomes infeasible. Thus, the model achieves } \\
\text { a balance between satisfying probability constraints and keeping cost } \\
\text { low. }\end{array}$ \\
$\begin{array}{c}\text { Disruptions in } \\
\text { the } \\
\text { Transportation } \\
\text { Network }\end{array}$ \\
\hline
\end{tabular}




\begin{tabular}{|c|c|}
\hline $\begin{array}{l}\text { Multi-suppliers } \\
\text { \& Perishable } \\
\text { Commodities }\end{array}$ & $\begin{array}{l}\text { Multi-suppliers/cross shipping and transportation of vital and } \\
\text { perishable commodities require extra attention while deciding on } \\
\text { safety stock levels. Planners and decision makers can use the model to } \\
\text { determine the additional safety stock needed for vital or perishable } \\
\text { commodities conducting a single-commodity analysis. Moreover, the } \\
\text { model enables one to order emergency supplies from other shelters or } \\
\text { suppliers, and to employ other means of transportation such as air } \\
\text { transportation using helicopters. }\end{array}$ \\
\hline Stochasticity & $\begin{array}{l}\text { The proposed framework can handle the unexpected fluctuations } \\
\text { likely to happen during emergency relief operations by introducing } \\
\text { white Gaussian noise into the system to better simulate the extreme } \\
\text { complexity expected under real-world emergency conditions. In } \\
\text { particular, for the scenarios studied, where the disruptions in the relief } \\
\text { system affect the flow of vital supplies to people located in shelters, it } \\
\text { takes longer for the model to achieve a comfortable convergence to the } \\
\text { optimal inventory levels. However, even in the worst-case scenario, } \\
\text { output inventory levels are still acceptable in such an unstable and } \\
\text { unpredictable disaster environment. }\end{array}$ \\
\hline $\begin{array}{l}\text { Real-life } \\
\text { Constraints }\end{array}$ & $\begin{array}{l}\text { Real-life constraints on inventory levels and supply flow increase } \\
\text { response time since imposing constraints on the system makes it } \\
\text { difficult for the mathematical model to converge. Therefore, additional } \\
\text { safety stock is needed to accommodate the need for vital supplies. } \\
\text { Planners and decision makers should select the levels of safety stock } \\
\text { accordingly to account for the long response time that is incurred due } \\
\text { to these limitations. }\end{array}$ \\
\hline $\begin{array}{c}\text { RFID } \\
\text { Integration }\end{array}$ & $\begin{array}{l}\text { Although limitations and difficulties exist as mentioned in Section5.3, } \\
\text { with a successful implementation, RFID systems provide a total } \\
\text { visibility of commodity movement in the disaster supply chain. By } \\
\text { feeding this data into SHIC model in terms of the amounts of } \\
\text { consumption and deliveries at the shelters, one can make early } \\
\text { decisions about pre-stocking inventories in case of possible } \\
\text { interruptions in the supply. Use of these accurate distributions within } \\
\text { SHIC model will result in obtaining accurate safety stocks, and } \\
\text { therefore achieving efficient planning for disaster inventories. This } \\
\text { planning information can then be used to feed the proposed online } \\
\text { methodologies to be able to facilitate real-time inventory level } \\
\text { tracking. }\end{array}$ \\
\hline $\begin{array}{c}\text { Applicability to } \\
\text { Different } \\
\text { Types of } \\
\text { Disasters }\end{array}$ & $\begin{array}{l}\text { The proposed integrated model can be used to examine the differences } \\
\text { in inventory strategies for different types of disasters as clearly } \\
\text { explained in Table } 6.1 .\end{array}$ \\
\hline
\end{tabular}




\subsubsection{Practical Implementations}

Although the proposed model in this thesis mostly highlights hurricane-based case studies, the methodology is capable of providing robust emergency inventory planning and management for different types of disasters. However, data should be obtained for specific disaster types to be able to implement the methodology successfully. Using the real-life data enhanced model, disaster case studies can be prepared by planners and emergency officials to plan for and respond to the uncertainties involved in disaster relief operations.

\subsubsection{Limitations and Realistic Suggestions to Deal with These Problems}

In our research, we have been involved in designing an emergency inventory management framework for disaster victims located at emergency shelters where we developed and used offline and online mathematical modeling approaches successfully in practice via realistic case studies. However, some questions from both a theoretical and a practical point of view are not adequately answered. Some of these questions are addressed in our current research or will be addressed in the near future. In this context, we mention questions relating to online tracking based on RFID devices, further validation of the mathematical model, and the sensitivity and robustness of the model. Based on these questions, limitations of this study include the following where some of these problems can be dealt with as presented below:

- First, depending on the severity of the disaster, there may not be a way of tracking the inventory levels and the flow of supplies. Absence of emergency officials 
(both at the inventories and shelters), most probably in the immediate aftermath of a disaster, will also cause problems related to online tracking with RFID devices.

- For these cases where the disaster conditions are highly unpredictable, the use of the online control model can be infeasible and representing the real world disaster conditions will not be possible.

- Tight limitations on inventory levels and the flow of supplies within the disaster relief system will cause instability and infeasibility for the online control model.

- For some extreme events like floods, low strength hurricanes or chemical explosions, the best course of action for people may be to take immediate shelter wherever they are located. This shelter may be the victim's house, or any other place the victim is close to when the incident occurs. In this case, if people are informed about the disaster beforehand (as in the case of Hurricane Irene), they are more likely to stock water and food for further use in the aftermath of the disaster. This issue is not considered in the current version of the proposed model. However, it is possible to cluster groups of houses and consider delivering vital supplies to these clusters where the total amount of commodities stocked beforehand in the whole cluster can represent the initial safety stock used in the model.

- The transportation network and its costs are not explicitly incorporated within the framework. In the model, the number of deliveries is used to represent the performance of the transportation system. Whenever the number of deliveries is not enough for the victims located at the shelters, we need additional safety stock, therefore an adjustment cost is incurred to satisfy the probabilistic constraint 
given in Equation (4.10). That is, there is an additional cost incurred within the model which directly depends on the number of deliveries and the transportation of vital supplies. On the other hand, explicit addition of the commodity transportation costs (using a real transportation network) into the model is an interesting area of future work since it will require a distance based clustering model that controls the location and allocation of the shelters and emergency inventories, and the flow of supplies from the inventories to shelters.

- Information loss within the disaster supply chain due to disruptions in the communication infrastructure or data security breaches is also a serious problem where online tracking becomes impossible. However, the receding horizon concept within the CMPC model offers a solution to this problem as the information from the last time step where information is available can always be used to supply the shelters until the communication within the supply chain is restored.

\subsubsection{Discussions of Alternative Approaches}

As an alternative to the analytical model presented in this study, agent-based approaches that can be implemented as a computer-based simulation model can also be employed to study the emergency inventory management problem. This alternative simulation-based framework should include the locations of shelters and inventories as well as the transportation network and information on the flow of supplies in the network. This type of system will also need data obtained from previous disasters for the model to be able to simulate real-life conditions accurately. Although this simulation-based model 
will enable planners and decision makers to work and test many scenarios, one should be careful about implementing the results. An agent-based approach does not allow one to model system dynamics analytically and to obtain an optimal solution for the overall inventory management problem (both offline and online) systematically. Moreover, the error expected to occur in the agent-based models that might be due to the assumptions of the simulation should be clearly identified. The authors, therefore, believe that the use of a mathematical approach is more appropriate than agent-based approaches in terms of being able to mathematically analyze the modeled dynamic system than just using a simulation that can be a black box. This black box might not allow us to interpret the output of the simulation in terms of convergence and optimality as well as realism. However, the proposed model in this thesis can also be used along with agent-based approaches to train the simulation or validate the outcomes of the simulation.

\subsection{Future Research Directions}

For future research on emergency inventory management, several directions are worthy of attempt.

First of all, the most important feature of the proposed framework is that it can be applied to any kind of disaster scenario with distinctive characteristics by adapting parameters of the offline and online models. However, to build even more realistic and comprehensive case studies and test our model using these, there is a need for better data collection from different real-life disasters. This type of study will show whether or not this model works properly and efficiently with regard to realistic disaster cases. Our future work, therefore, will involve validating our methodology using the real-life data 
obtained from emergency response teams. A pilot test involving RFID technologies to keep track of the dynamic change in inventory levels, and the flow of emergency vehicles and supplies can also be used to collect data that can be used for the training process.

A possible improvement on the speed and performance of the Prékopa-VizvariBadics algorithm in terms of computation time while solving the SHIC problem involves employing the bundle preprocessing method before finding the pLEP sets, which was proposed by Lejeune and Noyan (2010). A future direction for functional approximation is to develop guidelines to determine the optimal structure of the functional approximator, i.e., the number of hidden layers and nodes of a neural network, based on real data if available via RFID tracking or other sources. A similar future research direction is to develop guidelines for calculating optimal system control parameters for the consumption and delivery processes within the CMPC methodology. Studying different approaches for state-space system formulation and comparing the effects of these different models on system performance also requires extra attention.

The physical location of emergency inventories and shelters is of interest in terms of the logistical aspects of transporting emergency supplies. Therefore, integration of such a location and allocation module with the aforementioned framework would be a significant improvement to the proposed integrated emergency inventory management methodology. The direct addition of the commodity transportation cost into the model is also an interesting area for future work, as it will require a distance-based clustering model that controls the location and allocation of shelters and emergency inventories and the flow of supplies from these inventories to shelters. Addition of a budgetary constraint 
into the model is also promising for future work as it will enable us to perform a benefitcost analysis using the model.

\section{REFERENCES}

1. Afshar, A.M., and Haghani, A., (2008) "A heuristic framework for optimizing hurricane evacuation operations", Transportation Research Record, 2089, pp. 9-17.

2. Akkihal, A.R., (2006) Inventory pre-positioning for humanitarian operations, $M S$ Thesis, Massachusetts Institute of Technology, Boston, MA, USA.

3. Alcada-Almeida, L., Tralhao, L., Santos, L., and Coutinho-Rodrigues, J., (2009) “A multiobjective approach to locate emergency shelters and identify evacuation routes in urban areas", Geographical Analysis, 41, pp. 9-29.

4. Almutairi, N.B., and Zribi M., (2006) "Sliding mode control of coupled tanks", Mechatronics, 16, pp. 427-441.

5. Altay, N., Prasad, S., and Sounderpandian, J., (2009) "Strategic planning for international disaster relief logistics: Implications for research and practice", International Journal of Services Sciences, 2(2), pp. 142-161.

6. Amin, S., Cox, M., and Goldstein, M., (2008) "Using data against disasters: Overview and synthesis of lessons learned", pp. 1-22, World Bank Report on Data Against Natural Disasters, Edited by Amin S., and Goldstein, M., Washington D.C., USA.

7. Anderson, B., and Moore, J., (1979) Optimal Filtering, Prentice-Hall, New York, USA.

8. Apte, A., and Yoho, K.D., (2011) "Strategies for Logistics in Case of a Natural Disaster", 8th Annual Acquisition Research Symposium, Graduate School of Business \& Public Policy, Naval Postgraduate School, May 11-12, 2011, CA, USA.

9. Athukorala, P.C., and Resosudarmo, B.P., (2005) "The Indian ocean tsunami: Economics impact, disaster management and lessons", Working Paper in Trade and Development No. 2005/05. Division of Economics, Australian National University, Australia.

10. Azzalini, A., and Dalla Vella, A., (1996) "The multivariate skew-normal distribution", Biometrika, 83(4), pp. 715-726.

11. Balcik, B., Beamon, B.M., Krejci, C.C., Muramatsu, K.M., and Ramirez, M., (2010) "Coordination in humanitarian relief chains: Practices, challenges and opportunities", International Journal of Production Economics, 126, pp. 22-34.

12. Balcik, B., and Beamon B.M., (2008) "Facility location in humanitarian relief", International Journal of Logistics: Research and Applications, 11(2), pp. 101-121.

13. Bansal, K., Vadhavkar, S., and Gupta, A., (1998) "Neural networks based forecasting techniques for inventory control applications", Data Mining and Knowledge Discovery, 2, pp. 97-102. 
14. Bao, S. Lin, C., (2009) "Ant colony optimization control for fuzzy neural network in freeway entrance ramp", Journal of Transportation Information and Safety, 5(27), pp. 173-176.

15. Barbarosoglu, G., and Arda, Y., (2004) "A two-stage stochastic programming framework for transportation planning in disaster response", Journal of Operational Research Society, 55, pp. 43-53.

16. Barbarosoglu, G., Ozdamar L., and Cevik A., (2002) "An interactive approach for hierarchical analysis of helicopter logistics in disaster relief operations", European Journal of Operational Research, 140, pp. 118-133.

17. Barry, J.R., Lee, E.A., and Messerschmitt, D.G., (2004) Digital communication, Kluwer Academic Publishers, Massachusetts, USA.

18. BBC News, 2005. Implant chip to identify the dead, http://news.bbc.co.uk/2/hi/technology/4721175.stm.

19. Beamon, B.M., and Kotleba, S.A., (2006) "Inventory modeling for complex emergencies in humanitarian relief operations", International Journal of Logistics: Research and Applications, 9(1), pp. 1-18.

20. Beamon, B.M., and Balcik, B., (2008) "Performance measurement in humanitarian relief chains", International Journal of Public Sector Management, 21(1), pp. 4-25.

21. Berioli, M., Courville, N., and Werner, M., (2007) "Integrating satellite and terrestrial technologies for emergency communications: The WISECOM project", Proceedings of the International Conference on Heterogeneous Networking for Quality, Reliability, Security and Robustness, August 14 - 17, Vancouver, British Columbia, Canada.

22. Blanco, E.E., and Goentzel, J., (2006) "Humanitarian supply chains: A review", MIT Center for Transportation \& Logistics, POMS.

23. Bozorgi-Amiri, A., Jabalameli, M.S., Al-e-Hashem, S.M.J.M., (2011) "A multiobjective robust stochastic programming model for disaster relief logistics under uncertainty", OR Spectrum, DOI 10.1007/s00291-011-0268-x.

24. Braun, M.W., Rivera, D.E., Carlyle, W.M., and Kempf, K.G., (2003a) "A model predictive control framework for robust management of multi-product, multiechelon demand networks", Annual Reviews in Control, 27, pp. 229-245.

25. Braun, M.W., Rivera, D.E., Carlyle, W.M. and Kempf, K.G., (2003b) "Application of Model Predictive Control to Robust Management of Multiechelon Demand Networks in Semiconductor Manufacturing," Simulation, 79(3), pp. 139-156.

26. Brodie, M., Weltzien, E., Altman, D., Blendon, R.J., and Benson, J.M., (2006) "Experiences of hurricane Katrina evacuees in Houston shelters: Implications for future planning", American Journal of Public Health, 96(5), pp. 1402-1408.

27. Chang, M-S., Tseng, Y-L., Chen, J-W., (2007) "A scenario planning approach for the flood emergency logistics preparation problem under uncertainty", Transportation Research Part E, 43, pp. 737-754.

28. Chang, M-S., and Hsueh, C-F., (2007) "Developing geographic information system for flood emergency logistics planning", Proceedings of the 2007 Summer Computer Simulation Conference, San Diego, CA, USA. 652-659.

29. Chatfield, A., Wamba, S.F., and Hirokazu, T., (2010) "E-Government challenge in disaster evacuation response: The role of RFID technology in building safe and 
secure local communities", Presented at the 43th Hawaii International Conferences on System Sciences (HCISS), Hawaii, USA.

30. Chatfield, A., and Wamba, S.F., (2009) "RFID deployment in natural disaster management: Current focus \& future", Proceedings of the 5th International conference on Sensors, Sensor Networks and Information Processing (ISSNIP), Melbourne, Australia.

31. Childers, A.K., Visagamurthy, G., and Taaffe, K., (2009) "Prioritizing patients for evacuation from a healthcare facility", Presented at Transportation Research Board's 88th Annual Meeting, Washington D.C., USA.

32. Clark A.R. and Culkin B., (2007) "A network trans-shipment model for planning humanitarian relief operations after a natural disaster", Presented at EURO XXII, 22nd European Conference on Operational Research, Prague, Czech Republic.

33. Danistan, J., and Vipulanandan, C., (2010) "Artificial neural network approach to predict the traffic flow during neighborhood hurricane and other emergency evacuation", THC-IT Conference Proceedings, University of Houston, 2 pp.

34. Davidson, A.L., (2006) Key performance indicators in humanitarian logistics, $M S$ Thesis, Massachusetts Institute of Technology, Boston, MA, USA.

35. De Leeuw, S., Vis, I. F.A., and Jonkman, S.N., (2009) "Logistics aspects of emergency preparedness in flood disaster prevention", Serie Research Memoranda, 44, VU University Amsterdam, Faculty of Economics.

36. Dessouky, M., Ordonez F., Jia, H., and Shen. Z., (2006) "Rapid distribution of medical supplies", International Series in Operations Research and Management Science, 91, pp. 309-338.

37. Destro, L., and Holguin-Veras, J., (2011) "Material convergence and its determinants: The case of Hurricane Katrina", Presented at Transportation Research Board's 90th Annual Meeting, Washington D.C., USA.

38. Donahue, A.K., and Joyce P.G., (2001) "A framework for analyzing emergency management with an application to federal budgeting", Public Administration Review, 61(6), pp. 728-740.

39. Dunbar, W.B., and Desa, S., (2005) "Distributed model predictive control for dynamic supply chain management", International Workshop on Assessment and Future Directions of NMPC, Germany.

40. Duran, S., Gutierrez, M.A., and Keskinocak, P., (2011) "Pre-positioning of emergency items worldwide for CARE International", Interfaces, 41(3), pp. 223237.

41. EM-DAT, The International Disaster Database, Center for Research on the Epidemiology of Disasters (CRED), http://www.emdat.be.

42. Ergun, O., Keskinocak, P., and Swann, J., (2007) "Humanitarian relief logistics", Operations Research Management Science, 34(6), pp. 28-33.

43. Ezziane, Z.H., Mazouz, A.K., and Han, C., (1993) "Inventory control neural network system", Proceedings of the 25th IEEE Conference on System Theory, pp. 243-246, Tuscaloosa, AL, USA, March 07-09.

44. Friedrich, F., Gehbauer, F., and Rickers, U., (2000) "Optimized resource allocation for emergency response after earthquake disasters”, Safety Science, 35. pp. 41-57. 
45. Gadh, R., and Prabhu B.S., (2006) "Radio frequency identification of hurricane Katrina victims", IEEE Signal Processing, 23(2), pp. 184-185.

46. Haghani, A., and Oh, S., (1996) "Formulation and solution of a multi-commodity, multi-modal network flow model for disaster relief operations", Transportation Research Part A, 30, pp. 231-250.

47. Han, Y., Guan, X., and Shi, L., (2007) "Optimal supply location selection and routing for emergency", Proceedings of the IEEE Conference on Automation Science and Engineering, pp. 1039-1044, Scottsdale, AZ, USA, September 22-25.

48. Henderson, J.H., (2007) Logistics in support of disaster relief, AuthorHouse. Bloomington, Indiana, USA.

49. Holguin-Veras, J., Perez, N., Jaller, M., Destro, L., and Wachtendorf, T., (2010) "On the need to reformulate humanitarian logistics modeling: Deprivation costs, and material convergence", Presented at Transportation Research Board's 87th Annual Meeting, Washington D.C., USA.

50. Holguin-Veras, J., Wachtendorf, T., Ukkusuri, S., and Brown, B., (February 2006January 2008) Small grant for exploratory research: characterization of the supply chains in the aftermath of an extreme event: The Gulf coast experience, Funded by the National Science Foundation, NSF-CMMI No. 0554949, Collaborative Project of Disaster Research Center/University of Delaware and Rensselaer Polytechnic Institute.

51. Holguin-Veras, J., Perez, N., Ukkusuri, S., Wachtendorf, T., and Brown, B., (2007) "Emergency logistics issues affecting the response to Katrina: A synthesis and preliminary suggestions for improvement", Transportation Research Record, 2022, pp. 76-82.

52. Holguin-Veras, J., Jaller, M., Ukkusuri, S., Brom, M., Torres, C., Wachtendorf, T., and Brown, B., (2008) "An analysis of the temporal distribution of requests for critical supplies after hurricane Katrina", Presented at Transportation Research Board's 87th Annual Meeting, Washington D.C., USA.

53. Hostikka, S., Korhonen, T., Paloposki, T., Rinne, T., Matikainen, K., and Heliovaara, S., (2007) "Development and validation of FDS-Evac for evacuation simulations", VTT Research Notes, 2421, VTT Technical Research Centre of Finland, Finland.

54. Houming, F., Tong, Z., Xiaoyan, Z., Mingbao, J., Guosong, D., (2008) "Research on emergency relief goods distribution after regional natural disaster occurring", Proceedings of the International Conference on Information Management and Industrial Engineering, 3, pp. 156-161.

55. Hwang, H-S., (1999) “A food distribution model for famine relief”, Computers \& Industrial Engineering, 37, pp. 335-338.

56. Iakovou, E., and Douligeris, C., (2001) "An information management system for the emergency management of hurricane disasters", International Journal of Risk Assessment and Management, 2(3/4), pp. 243-262.

57. Islam, M., Hannan, M.A., Samad, S.A., and Hussain, A., (2009) "Software defined radio for RFID application", Proceedings of the World Congress on Engineering and Computer Science (WCECS), San Francisco, USA. 
58. Iusem, A.N., and De Pierro, A.R., (1990) "On the convergence properties of Hildreth's quadratic programming algorithm", Mathematical Programming, 47, pp. 37-51.

59. Jaller, M., Ukkusuri, S., and Holguin-Veras, J., (2008) "A stochastic inventory model for fixed lifetime goods for disaster planning", Presented at Transportation Research Board 87th Annual Meeting, Washington D.C., USA.

60. Jia, H., Ordonez, F., and Dessouky, M., (2007) "A modeling framework for facility location of medical services for large-scale emergencies", IIE Transactions, 39, pp. 41-55.

61. Kanchanasut, K., Tunpan, A., Awal, M.A., Das, K., Wongsaardsakul, T., and Tsuchimito, Y., (2007) DUMBONET: A multimedia communication system for collaborative emergency response operations in disaster affected areas, International Journal of Emergency Management, 4(4), pp. 670-681.

62. Kirsch, T., Goldman, L., Rowel, R. Foushee, H. R., Rodgers, J., Ravndal, D., and Temdrup, T., (2006-continued) Surge capacity networks, A collaborative PACER (National Center for the Study of Preparedness and Catastrophic Event Response) project of Johns Hopkins University, American Red Cross, University of AlabamaBirmingham, Morgan State University, University of South Florida, and Penn State University, PACER, DHS (USDHS (N00014-06-0991)).

63. Kongsomsaksakul, S., Yang, C., and Chen, A., (2005) "Shelter location-allocation model for flood evacuation planning", Journal of the Eastern Asia Society for Transportation Studies, 6, pp. 4237 - 4252.

64. Kovacs, G., and Spens, K.M., (2007) "Humanitarian logistics in disaster relief operations", International Journal of Physical Distribution \& Logistics Management, 37(2), pp. 99-114.

65. Kovacs, G., and Spens, K.M., (2011) "Humanitarian logistics and supply chain management: the start of a new journal", Journal of Humanitarian Logistics and Supply Chain Management, 1(1), pp. 5-14.

66. Kwon, W.H., and Han, S., (2005) Receding horizon control: Model predictive control for state models, Springer-Verlag London Limited, London, England.

67. Lee, Y. W., (1960) Statistical Theory of Communication, Wiley, New York.

68. Lee, Y. M., Ghosh S., and Ettl, M., (2009) "Simulating distribution of emergency relief supplies for disaster response operations", Proceedings of the 2009 Winter Simulation Conference, pp. 2797-2808.

69. Lejeune, M., and Noyan, N. (2010) "Mathematical programming approaches for generating p-efficient points", European Journal of Operational Research, 207, pp. 590-600.

70. Leonard, D. (2005) “The only lifeline was Walmart”, Fortune, 152(7), pp. 74-80.

71. Levenberg, K., (1944) "A method for the solution of certain non-linear problems in least squares", The Quarterly of Applied Mathematics, 2, pp. 164-168.

72. Li, L., and Tang, S., (2008) "An artificial emergency-logistics-planning system for severe disasters", IEEE Intelligent Systems, 23(4), pp. 86-88.

73. Lin, Y-H., Batta, R., Rogerson, P.A., Blatt, A., and Flanigan M., (2010) "Application of a humanitarian relief logistics model to an earthquake disaster", 
Presented at Transportation Research Board 89th Annual Meeting, Washington D.C., USA.

74. Litman, T., (2006) "Lessons from Katrina and Rita: What major disasters can teach transportation planners", Journal of Transportation Engineering, 132, pp. 11-18.

75. Liu, H.X., Ban, X.J., Ma, W., and Mirchandani, P.B., (2007) "Model reference adaptive control framework for real-time traffic management under emergency evacuation”, Journal of Urban Planning and Development, 133(1), pp. 43-50.

76. Lodree, Jr., E.J., (2011) "Pre-storm emergency supplies inventory planning", Journal of Humanitarian Logistics and Supply Chain Management, 1(1), pp. 50-77.

77. Long, D.C., and Wood, D.F., (1995) “The logistics of famine relief”, Journal of Business Logistics, 16(1), pp. 213-229.

78. Marquardt, D., (1963) "An algorithm for least-squares estimation of nonlinear parameters", SIAM Journal of Applied Mathematics, 11, pp. 431-441.

79. Mei, B., (2002) Development of trip generation models of hurricane evacuation, $M S$ Thesis, Louisiana State University, Baton Rouge, LA, USA.

80. Mete, H.O., and Zabinsky, Z.B., (2010) "Stochastic optimization of medical supply location and distribution in disaster management", International Journal of Production Economics, 126, pp. 76-84.

81. Minyard, E., (2007) "The use of RFID in emergency management planning", Disaster Recovery Journal, 20, pp. 28-30.

82. Narendra, K.S., and Parthasarathy, K., (1990) "Identification and control of dynamical systems using neural networks", IEEE Transactions on Neural Networks, 1, pp. 4-26.

83. National Institute of Standards and Technology (NIST), (2011) Technical Study on Impacts of Joplin, Mo., Tornado, ongoing project, Gaithersburg, MD.

84. National Research Council of the National Academies, (2007a) Successful response starts with a map: Improving geospatial support for disaster management, National Academies Press, Washington D.C., USA.

85. National Research Council of the National Academies, (2007b) Improving disaster management: The role of IT in mitigation, preparedness, response, and recovery, National Academies Press, Washington D.C., USA.

86. National Research Council of the National Academies, (2005) Workshop on using information technology to enhance disaster management, Computer Science and Telecommunications Board.

87. News.com, (2005) RFID Chips Used to Track Dead after Katrina, http://news.cnet.com/RFID-chips-used-to-track-dead-after-Katrina/2100-11390_35869708.html

88. Nigg, J.M., Barnshaw, J., and Torres, M. R., (2006) "Hurricane Katrina and the flooding of New Orleans: Emergent issues in sheltering and temporary housing", The ANNALS of the American Academy of Political and Social Science, 604(1), pp. 113-128.

89. Noji, E.K., (1997) "The nature of disaster: General characteristics and public health effects", The Public Health Consequences of Disasters, edited by Eric K. Noji, Oxford University Press, pp. 3-20. 
90. Nouali, N., Bouchama, N., Bendjoudi, A., Babakhouya, A., Yahiaoui, S., Belhoul, Y., Zeghilet, H., and Guellati, N., (2009) "Information technology for enhancing disaster management", Presented at Congrès National des Télécommunications et leurs Applications (CNTA' 09), May, 23-24, University of Bejaia, Algeria.

91. Noyan, N., and Prékopa A., (2006) "A variant of the hungarian inventory control model", International Journal of Production Economics, 103-2, pp. 784797.

92. Oh, S., and Haghani, A., (1997) "Testing and evaluation of a multi-commodity multi-modal network flow model for disaster relief management", Journal of Advanced Transportation, 31(3), pp. 249-282.

93. Ozbay, K., and Ozguven, E.E., (2007) "Stochastic humanitarian inventory control model for disaster planning", Transportation Research Record, 2022, pp. 63-75.

94. Ozdamar, L., Ekinci, E., and Kucukyazici, B., (2004) "Emergency logistics planning in natural disasters", Annals of Operations Research, 129, pp. 217-245.

95. Ozdamar L., and Yi, W., (2008) "Greedy neighborhood search for disaster relief and evacuation logistics", IEEE Intelligent Systems, 23(1), pp. 14-23.

96. Ozguven, E.E., and Ozbay, K., (2008) "Simultaneous perturbation stochastic approximation algorithm for solving stochastic transportation network analysis problems: Performance evaluation”, Transportation Research Record, 2085, pp. 1220 .

97. Ozguven, E.E., and Ozbay, K., (2009) "A secure and efficient emergency management system for disasters", Proceedings of the International Conference on Models and Technologies for Intelligent Transportation Systems, pp. 298-305, June 22-23, Rome, Italy.

98. Ozguven, E.E. and Ozbay, K., (2011) "A secure and efficient inventory management system for disasters", Transportation Research Part C, doi:10.1016/j.trc.2011.08.012.

99. Ozguven, E.E. and Ozbay, K., (2012) "Case Study Based Evaluation of a Stochastic Multi-Commodity Emergency Inventory Management Model”, Accepted for Presentation in Transportation Research Board's $91^{\text {th }}$ Annual Meeting, Washington, D.C., USA.

100. Pao, Y.M., Phillips, S.M., and Sobajic, D.J., (1992) "Neural-net computing and the intelligent control of systems", International Journal of Control, 56, pp. 263-289.

101. Peng, X., Mo, Z., Laisheng, X., and Liao, R., (2011) "Rank control system for traffic artery based on wireless sensor and fuzzy neural network", Communications in Computer and Information Science, 158(7), pp. 438-444.

102. Prékopa, A., (1971) "Logarithmic concave measures with applications to stochastic programming”, Acta Scientiarum Mathematicarum, 32, pp. 301-316.

103. Prékopa, A., (1990) "Dual method for a one-stage stochastic programming problem with random RHS obeying a discrete probability distribution", ZOR-Methods and Models of Operations Research, 34, pp. 441-461.

104. Prékopa, A., (2003) Probabilistic programming, in: A. Ruszczynski and A. Shapiro, eds., Handbooks in operations research and management science, 10, Elsevier, New York, USA, pp. 267-351. 
105. Prékopa, A., (2006) "On the hungarian inventory control model”, European Journal of Operational Research, 171, pp. 894-914.

106. Prékopa, A., (2010) "Multivariate value at risk and other topics", Annals of Operations Research, online first, DOI 10.1007/s10479-010-0790-2.

107. Rasku, H., Rantala, J., and Koivisto, H., (2004) "Model reference control in inventory and supply chain management", First International Conference on Informatics in Control, Portugal.

108. Ray, J., (1987) A multi-period linear programming model for optimally scheduling the distribution of food-aid in West Africa, MS Thesis, University of Tennessee, Knoxville, TN, USA.

109. Rawls, C.G., and Turnquist, M.A., (2010) "Pre-positioning of emergency supplies for disaster response", Transportation Research Part B, 44, pp. 521-534.

110. Renne, J.L., Sanchez, T., Jenkins, P., and Peterson, R., (2009) "The challenge of evacuating the carless in five major U.S. cities: Identifying the key issues being faced", Presented at Transportation Research Board's 88th Annual Meeting, Washington D.C., USA.

111. Ripley, B.D., (1996) Pattern recognition and neural networks, Cambridge University Press, Cambridge, United Kingdom.

112. Robbins, H,. and Munro, S., (1951) "A stochastic approximation method", The Annals of Mathematical Statistics, 22(3), pp. 400-407.

113. Rodman, W.K., (2004) Supply chain management in humanitarian relief logistics, MS Thesis, Department of the Air Force, Air University, Air Force Institute of Technology, Wright-Patterson Air Force Base, Ohio, USA.

114. Russell, T.E., (2005) The humanitarian relief supply chain: Analysis of the 2004 south east Asia earthquake and tsunami, MS Thesis, Massachusetts Institute of Technology Boston, MA, USA.

115. Sahana FOSS Disaster Management System Project, (2004-continued) Lanka Software Foundation, http://www.sahana.lk.

116. Sanderson, N.C., Skjelsvik, K.S., Drugan, O.V., Puzar, M., Goebel, V., MuntheKaas, E., Plagemann, T., (2007) "Developing mobile middleware-an analysis of rescue and emergency operations", Technical Report 358, Department of Informatics, University of Oslo, Norway.

117. Sartori, M.A., and Antsaklis, P.J., (1992) "Implementation of learning control systems using neural networks", IEEE Control Systems Magazine, 12, pp. 49-57.

118. Schwartz, J.D., Wang, W., and Rivera, D.E., (2006) "Simulation-based optimization of process control policies for inventory management in supply chains", Automatica, 42, pp. 1311-1320.

119. Schwartz, J.D., and Rivera, D.E., (2010) "A process control approach to tactical inventory management in production-inventory systems", International Journal of Production Economics, 125, pp. 111-124.

120. Scurfield, R.M., (2006) "Post-Katrina aftermath and helpful interventions on the Mississippi Gulf coast”, Traumatology, 12, pp. 104-120.

121. Shen, Z.M., Pannala, J., Rai, R., and Tsoi, T.S., (2009) "Modeling transportation networks during disruptions and emergency evacuations", Presented at Transportation Research Board's 88th Annual Meeting, Washington D.C., USA. 
122. Shen, G, and Kong, X., "Study on road network traffic coordination control technique with bus priority", IEEE Transactions on Systems, Man, and Cybernetics, Part C: Applications and Reviews, 39(3), pp. 343-351.

123. Sherali, H.D., Carter, T.B., and Hobeika, A.G., (1991) "A location/allocation model and algorithm for evacuation planning under hurricane/flood conditions", Transportation Research Part B, 25(6), pp. 439-452.

124. Sheu, J-B., (2007) "Challenges of emergency logistics management", Transportation Research Part E, 43, pp. 655-659.

125. Sourirajan, K., Ramachandran, B., and An, L., (2008) "Application of control theoretic principles to manage inventory replenishment in a supply chain", International Journal of Production Research, 46(21), pp. 6163-6188.

126. Spall, J.C., (1992) "Multivariate stochastic approximation using a simultaneous perturbation gradient approximation", IEEE Transactions on Automatic Control, 37(3), pp. 332-341.

127. Spall, J.C., (1998) "Model-free control of nonlinear stochastic systems with discrete-time measurements", IEEE Transactions on Automatic Control, 43(9), pp. 1198-1210.

128. Spall, J.C., and Cristion, J.A., (1997) ", A neural network controller for systems with unmodeled dynamics with applications to wastewater management", IEEE Transactions on Systems, Man, and Cybernetics, Part B: Cybernetics, 27(3), pp. 369-375.

129. Special Needs Evacuation Tracking System (SNETS), (2008) Medical Special Toolkit, Department of State Health Services, The State of Texas, Tab J and H.

130. Tadokoro, S., Matsuno, F., Asama, H., Onosato, M., Osuka, K., Doi, T., Nakanishi, H., Yokokohji, I., and Murata, M., (2007) DDT Project: Background and overview, Workshop on Rescue Robotics - DDT Project on Urban Search and Rescue, IEEE/RSJ07 International Conference on Intelligent Robots and Systems, pp. 1-22, San Diego, CA, USA.

131. Taskin, S., Lodree, E.J. Jr., (2010) "Inventory decisions for emergency supplies based on hurricane count predictions", International Journal of Production Economics, 126, pp. 66-75.

132. The Global Disaster Information Network, (1997) Harnessing information and technology for disaster management, Disaster Information Task Force Report, Washington D.C., USA.

133. The UNISDR Terminology on Disaster Reduction Report, UNISDR (United Nations International Strategy for Disaster Reduction), Geneva, Switzerland, 2009.

134. Tomaszewski, B.M., MacEachren, A.M., Pezanowski, S., Liu, X., and Turton, I., (2006) "Supporting humanitarian relief logistics operations through online geocollaborative knowledge management", Presented at the National Conference on Digital Government Research, May 21-25, San Diego, CA, USA.

135. Troy, D.A., Carson, A., Vanderbeek, J., and Hutton, A., (2007) "Enhancing community-based disaster preparedness with information technology", Disasters, 32(1), pp. 149-165. 
136. Tzeng, G-H., Cheng, H-J., and Huang, T.D., (2007) "Multi-objective optimal planning for designing relief delivery systems", Transportation Research Part E, 43, pp. 673-686.

137. van Wyk, E., (2010) Strategic Inventory Management for Disaster Relief, BS Thesis, University of Pretoria, South Africa.

138. Vilamovska, A-M., Hatziandreu, E., Schindler, H.R., van Oranje-Nassau, C., de Vries, H., and Krapels, J., (2009) "Study on the requirements and options for RFID application in healthcare identifying areas for radio frequency identification deployment in healthcare delivery: A review of relevant literature", Technical Report, RAND Cooperation, Santa Monica, CA, USA.

139. Wang, L., and Cluett, W. R. (2000) From plant data to process control, Taylor and Francis Inc., London, England.

140. Wang, L., (2001) "Continuous time model predictive control design using orthonormal functions”, International Journal of Control, 74(16), pp. 1588-1600.

141. Wang, L., Smith, S., and Chessari, C., (2008) "Continuous-time model predictive control of food extruder”, Control Engineering Practice, 16, pp. 1173-1183.

142. Wang, L., (2009) Model predictive control system design and implementation using $M A T L A B$, Advances In Industrial Control, Springer-Verlag London Limited, London, England.

143. Wang, L., Freeman, C.T., Rogers, E., and Owens, D.H., (2010) "Experimentally validated continuous-time repetitive control of non-minimum phase plants with a prescribed degree of stability", Control Engineering Practice, 18, pp. 1158-1165.

144. Wang, W., Rivera, D.E., and Kempf, K.G., (2007) "Model predictive control strategies for supply chain management in semiconductor manufacturing", International Journal of Production Economics, 107, pp. 56-77.

145. Waugh, W.L. Jr., (2000) Living with hazards, dealing with disasters: An introduction to emergency management, Armonk, N.Y., USA.

146. Webb P., (2005) "Food and nutrition concerns in Aceh after the tsunami", Food and Nutrition Bulletin. 26(4), pp. 393-396.

147. Whybark, D.C., (2007) "Issues in managing disaster relief inventories", International Journal of Production Economics, 108, pp. 228-235.

148. Widener, M.J., and Horner, M.W., (2011) "A hierarchical approach to modeling hurricane disaster relief goods distribution", Journal of Transport Geography, 19(4), pp. 821-828.

149. Wilmot, C.G., and Mei, B., (2004) "Comparison of alternative trip generation models for hurricane evacuation", Natural Hazards Review, 5, pp. 170-178.

150. Wismer, D.A., and Chattergy, R., (1978) Introduction to Nonlinear Optimization, Elsevier North-Holland, Inc., New York, NY, USA.

151. Wolshon, B., Urbina, E., Wilmot, C., and Levitan, M., (2005) "Review of policies and practices for hurricane evacuation I: Transportation planning, preparedness, and responses", Natural Hazards Review, 6(3), pp. 129-142.

152. Wong, Y. F., (2006) "Use of RFID for automatic baggage handling \& reconciliation at HKIA", Presented for Technical Services and Procurement Airport Authority, Hong Kong. 
153. Xie, C., and Turnquist, M., (2009) "Integrated evacuation network optimization and emergency vehicle assignment", Presented at Transportation Research Board 88th Annual Meeting, Washington D.C., USA.

154. Yang, N., and Federgruen A., (2006) "Safeguarding strategic supplies: Selecting an optimal set of suppliers", DOTM Colloquium Series, UCLA Anderson School of Management, Los Angeles, CA.

155. Yazici, M.A., and Ozbay, K., (2007) "Impact of probabilistic road capacity constraints on the spatial distribution of hurricane evacuation shelter capacities", Transportation Research Record, 2022, pp. 55-62.

156. Yi, W., and Ozdamar, L., (2007) "A dynamic logistics coordination model for evacuation and support in disaster response activities", European Journal of Operational Research, 179(3), pp. 1177-1193.

157. Yi, W., and Kumar, A., (2007) "Ant colony optimization for disaster relief operations", Transportation Research Part E, 43, pp. 660-672.

158. Yushimito, W.F., Jaller, M., and Ukkusuri, S., (2009) "Facility location on disasters: A voronoi based heuristic algorithm with an application to hurricane Katrina", Presented at Transportation Research Board's 88th Annual Meeting. Washington D.C., USA.

159. Zhan, H.M., and Ritchie, S.G., (1997) "Freeway ramp metering using artificial neural networks", Transportation Research Part C, 5(5), pp. 273-286. 


\section{CURRICULUM VITAE}

\section{Eren Erman Ozguven}

\section{Education}

2006-2012 Ph.D., Civil and Environmental Engineering, Rutgers University

2002-2006 Master of Science, Industrial Engineering, Bogazici University, Turkey

1997-2002 Bachelor of Science, Civil Engineering, Bogazici University, Turkey

\section{Publications}

\section{Journal Papers}

- Ozbay, K., Yazici, M. A., Iyer, S., Li, J, Ozguven, E. E., and Carnegie, J., (2012) "Use of a Regional Transportation Planning Tool for Modeling of Emergency Evacuation: A Case Study of Northern New Jersey", Accepted for Publication in Transportation Research Record.

- Ozguven, E.E, and Ozbay, K., (2012) "Case Study Based Evaluation of a Stochastic Multi-Commodity Emergency Inventory Management Model", Accepted for Publication in Transportation Research Record.

- Ozguven, E. E., Ozbay, K., (2011) "A Secure and Efficient Inventory Management System for Disasters", Transportation Research Part C, doi:10.1016/j.trc.2011.08.012.

- Ozbay, K., Ozguven, E. E., Sertel, T., Aboobaker, N., Littleton, B., and Caglar, K. (2009) "Manual of Guidelines for Inspection and Maintenance of Intelligent Transportation Systems", Transportation Research Record: Journal of Transportation Research Board, Volume 2129, pp. 90-100.

- Ozguven, E. E., and Ozbay, K., (2008) "Simultaneous Perturbation Stochastic Approximation Algorithm for Solving Stochastic Transportation Network Analysis Problems: Performance Evaluation", Transportation Research Record: Journal of Transportation Research Board, Volume 2085, pp. 12-20. 
- Ozguven, E. E., and Ozbay, K., (2008) "Nonparametric Bayesian Estimation of Freeway Capacity Distribution from Censored Observations", Transportation Research Record: Journal of Transportation Research Board, Volume 2061, pp. 2029.

- Ozbay, K., and Ozguven, E. E., (2007) "Stochastic Humanitarian Inventory Control Model for Disaster Planning", Transportation Research Record: Journal of Transportation Research Board, Volume 2022, pp. 63-75. 